ist

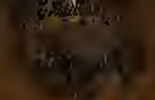

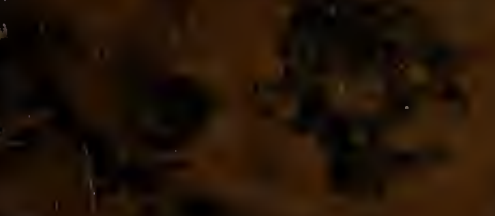

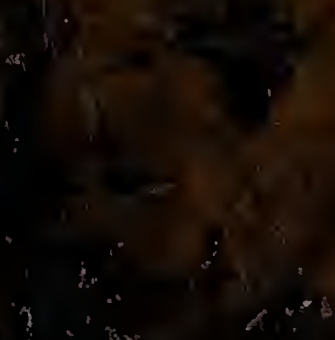

$\because \sin ^{2} ;$

th

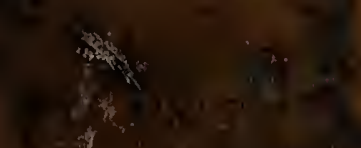

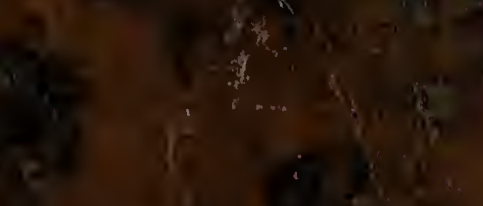

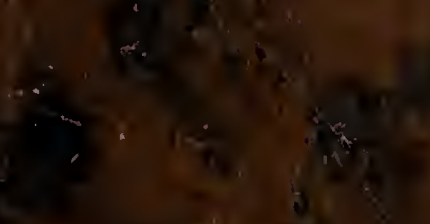

$$
\begin{aligned}
& -4 x-4
\end{aligned}
$$

$\rightarrow$$$
\therefore-x^{2}+x^{2}=
$$

$i^{3}=$

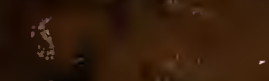

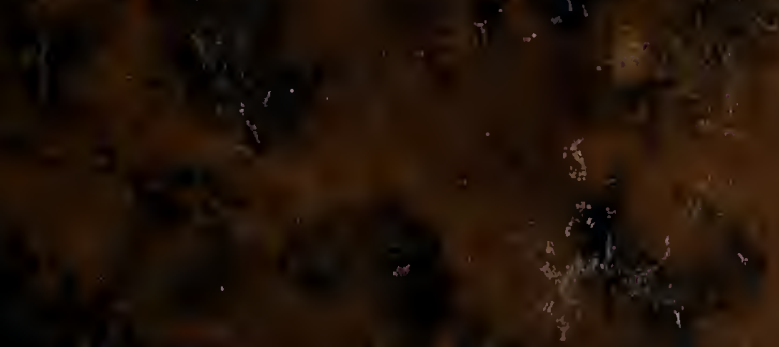
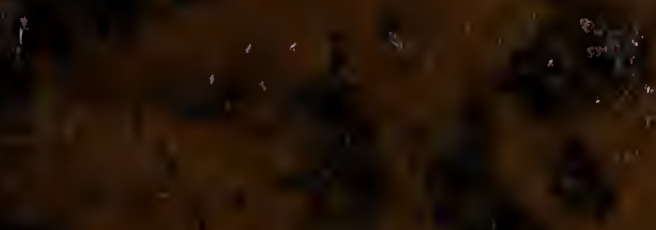

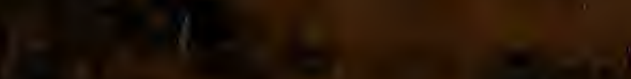

is,
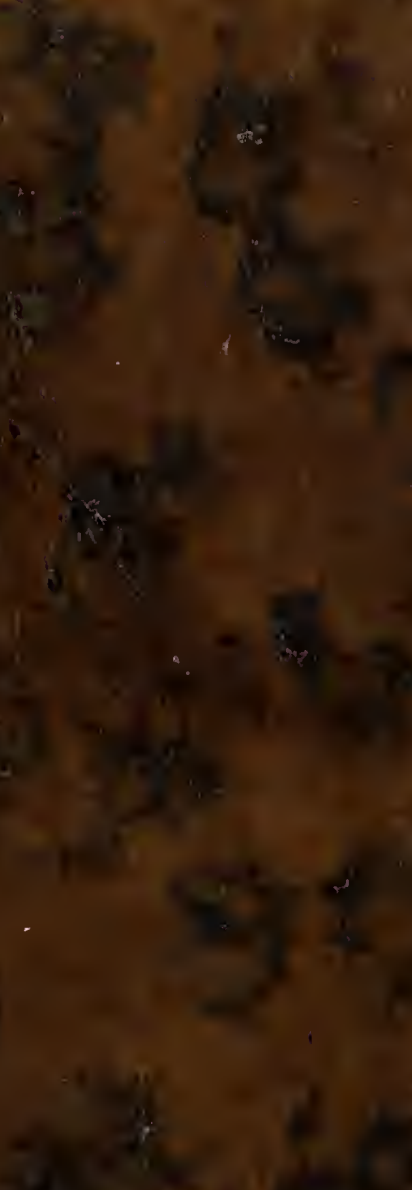

4 
12

10 ... $1 \%$ $-\infty .6$ - $600^{\circ}$ $1) f^{\circ}$ (1..1 Ii. N a pla ) ape - 18 o. 1
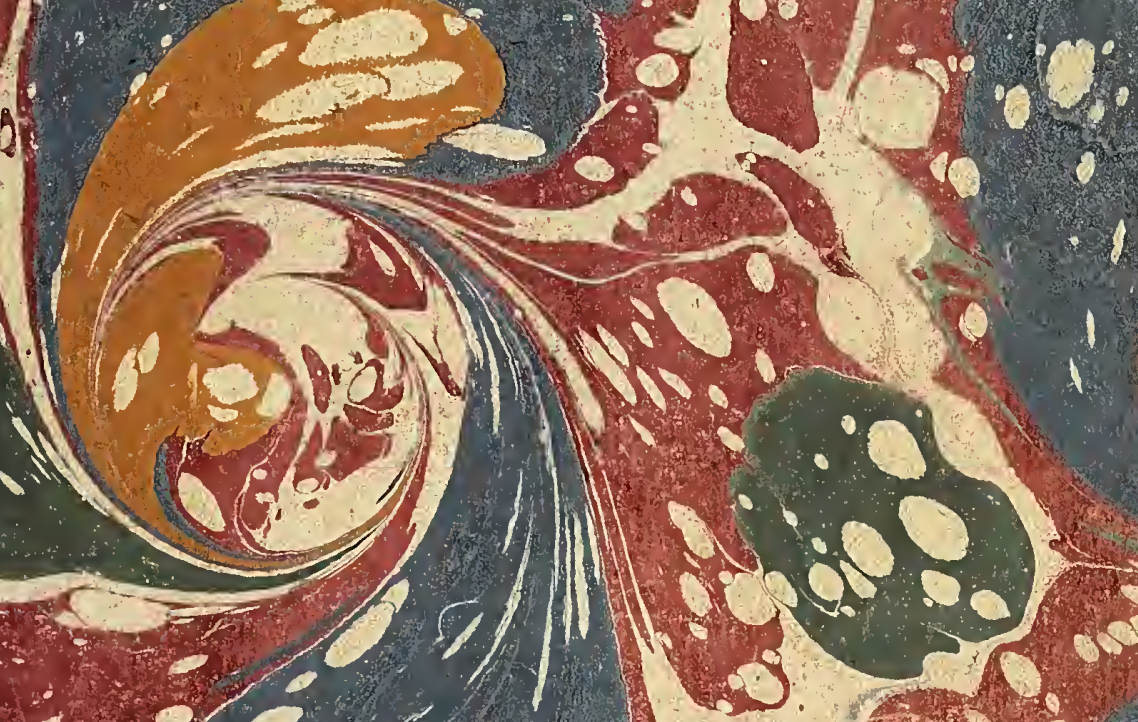

$12-10$

L.
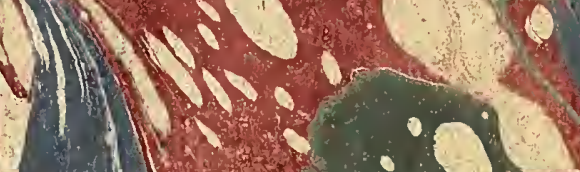
i

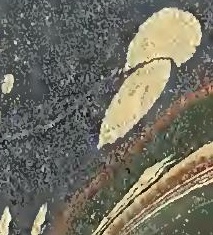


<smiles>CC=CC</smiles> 
$\lambda$ 
LIBRARIE SCIENTIFIQUE ANCIENNE ET MODERNE

EMILE BLANCHARD

I0, Rue de la Sorbonne, PARIS VENTE, ACHAT ECHANGE

de Livres Neufs et d'Occasion

COMHISSION-EXPORTÁTION - RELIURE 

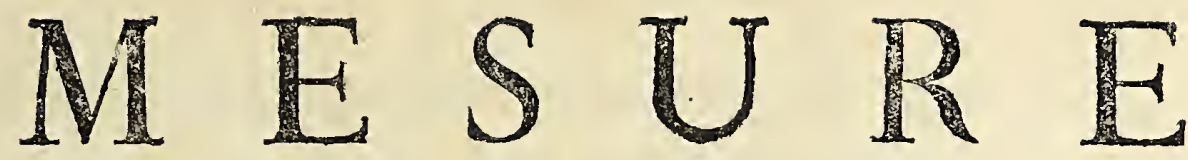

D E S

TROIS PREMIERS DEGRE'S DU

$M E ' R I D I E N$

DANS L'HE'MISPHERE AUSTRAL,

Tirée des Obfervations de M.rs de l'Académie Royale des Sciences, Envoyés par le Roi fous l'E'quateur:

Par M. DE La Condamine.

\section{Fuit alter}

Defcripfit radio medium qui gentibus Orbem. Virgil.

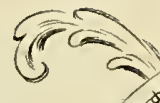

(2)
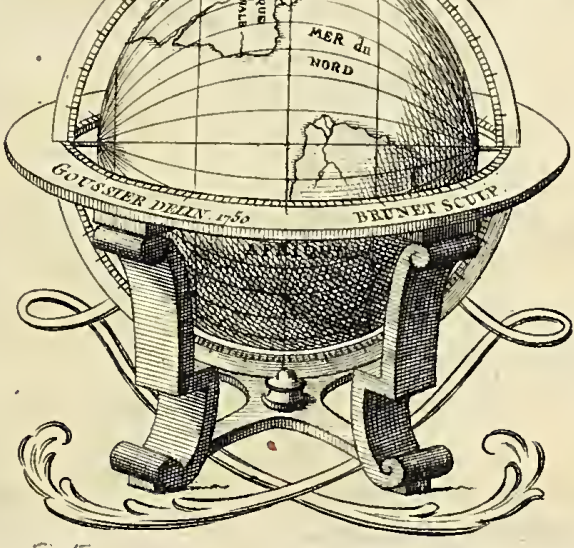

A PARIS,

DE L'IMPRIMERIE ROYALE. 



\section{A V E R T ISS E M E N T.}

T'IMPRESSION de ce Livre avoit été commencée 1 au mois de Mai 1749, fous la forme in-8. Les Tables des Triangles étoient imprimées \& toutes les Planches gravées, lorfqu'on recommença quelques mois après à l'Imprimerie Royale, la préfente édition in-4. qui n'a été finie qu'au mois de Mai I 75 o. Elle devoit paroître alors précédée d'une Introduction hiftorique fort Juccinte. Divers retardemens furvenus ont donné le temps à l'A uteur d'étendre cette. Introduction, $\dot{\mathcal{O}}$ d'en faire un Journal du Voyage académique à I'E'quateur. Cet ouvrage eft actuellement fous prefe; cependant on n'a pas cru devoir différer plus. longtemps la publicaiion de ce qui regarde la Mefure des degrés du Méridien, en attendant la partie hiftorique qui Juivra celle-ci de près.

Fautes à corriger.

Pages. : Lignes. Fautes.

$6, \quad 28$, auftral

ibid. 29, $C$

9, 21, dont

10, 15, près

14, 4, ceux-ci

I7, 29, cette

38,20 , avec

40, 5, voit

40,23 , en France
Corrections,

boréal

K

de la longueur duquel

plus ces deux Acadénuiciens une femblable

Par voient ajoûtez dans un ouvrage femblable au nôtre 


\begin{tabular}{|c|c|c|}
\hline Pages. & Lignes. & Fautes. \\
\hline $4 r$ & 21 & après nos \\
\hline 42, & 22, & cet enciroit \\
\hline 44, & g, & $\begin{array}{l}\text { toutes les corrections précé- } \\
\text { dentes }\end{array}$ \\
\hline 45 , & 26 , & obfervé \\
\hline 47, & II & $\&$ \\
\hline ibid. & 12, & je ne pouvois \\
\hline St, & $I$, & de l'angle \\
\hline 65 & 7 & TOD \\
\hline 67, & 14, & donnent \\
\hline 74,2 & $6 \delta 27$, & au centre de \\
\hline So, & 9, & augmentoit ou diminuoit \\
\hline 82, & 3 , & terme boréal \\
\hline 89, & 7 & $\times \varepsilon$ \\
\hline $9 z$ & 18, & angles, des erreurs \\
\hline 123 & $I I$, & auftrale \\
\hline 125, & IS, & à la fin \\
\hline & 23 & $\begin{array}{l}\text { moindre qu'elle ne parut } \\
\text { en } 1739^{\circ}\end{array}$ \\
\hline
\end{tabular}

Corrections.

après la mefure de nos

certe pointe

les trois premières corrections indiquées

conclu d'un angle obfervé

rayez \&

ne pouvant

du demi-angle

TOT

donnent pour réfultat

aux deux centres des

diminuoit ou augmentoit

(terme boréal)

$x \varepsilon$ \&c.

triangles, des erreurs

feptentrionale

Ic 20

moindre en 1739 qu'en

I 741, 1742 \& 1743 ,

toute réduction faite

Planche III

$A C P$

fléchi

diftance apparente

cette

diffèrent à peine de $2^{\prime \prime}$

vers le Sud au r. ${ }^{\text {er Janvier }}$

1743 .

en I 741 , je n'ai janaais vû alors ni depuis

devoit être accourcie

$205, \quad 3$, étoit trop courte

Planclie I, frg. 10.

ibid. $27, \quad$ après le mot réticule il faut. un renvoi $*$ à la note cià côté, qui a été oubliée au bas de la page.

234, 8 , 2. toifes

239, 3, Mefure aftronomique

248,6 , telle

$257, \quad I, \quad$ réfulte

264, pénulriène. Tit. II I
* On fuppofe ici que l'image dé l'étoile eft vîe an centre de la Lunette; \& dans mes dernières obfervations, elle en étoit fi près, que la difance au centre a pú être regardée comme nulle.

18 toifes.

Mefures

telles

ne réfulte

Lib. II I. 


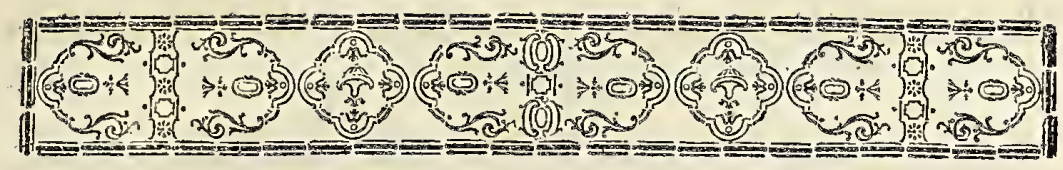

\section{TABLE DES ARTICLES.}

\section{Division de louvrage.}

PREMIERE PARTIE.

Mefure géométrique de l'arc du Méridien, ou opérations fur le terrein, pour fixer la pofition, do déterminer la longueur de la Ligne méridienne.

page 3

Article I. Mefure actuelle de la première Bafe des Triangles de la Méridienne aux environs de Quito, dans la plaine d'Y arouqui. 4

Article II. Du Syftène de Triangles, formé pour mefurer la Méridienne.

I O

Article 11I. Remarques fur les deux différentes Suites de Triangles, formées ponir la meflure de la Miridlienne. Nombre des Obfervateurs \& des Inftrumens qui y out été employés. $\quad$ I 2

Article IV. De ma Mefure géométrique particulière. I 3

ARTICLE V. Des différentes corrections faites aux angles objervés.

16

Article VI. Table des Triangles de la Méridienne de Quito.

$2 \mathrm{I}$

Article VII. Explication de la Colone I de la Table: Ordre \& Plan des Triangles. 


\section{T A B L E.}

Article VIII. Explication de la Colonne II: Noms des lieux ou étoient placés les Signaux. ibid.

Article IX. Explication de la Colonne 111: Angles de pofition objervés.

Art ICle X. Explication de la Colonne IV: Équation pout la fomme des trois angles. $\quad 44$

Article XI. Explication de la Colonne $V$ : Longueur des côtés oppofés aux angles objervés.

Article XII. Explication de la Colonne VI: Angles verticaux, ou de hauteur do de déprefjion apparente, réciproquement obfervés d'm Signal. à l'autre.

A r т. XIII. Explication de la Colonne VII: Hauteurs or abaiffemens refpectifs des Signawx.

49

Ant. XIV. Hauteurs abfolues des Signaux de la Méridieme, $\&$ des montagnes principales de la Province de Quito.

A R т. X V. Explication de la Colonne VIII: De la réduction des angles obfervés en différens plans, à Thorizon.

57.

A R T. XVI. Explication de la Colonne IX: Longueur des. côtés horizontaux, réduits all niveau de $\mathrm{Ca}$ rabourou.

59

A r T. XVII. Explication de la Colonne $X$ : Direction des côtés des Triangles par rapport à la Ligne méridienme.

ART. XVIII. Explication des Colonnes XI \& XII de le Table : Diftances entre les Méridiens les Paralleles des Signaux. 


\section{T A B L E.}

ART. XIX. Détermination des points des Triangles de la Méridienne à l'egard de Quito. 66

A r т. XX. Mefure de la Bafe de Tarqui.

$\mathrm{ART}$. XXI. Expériences fur les changemens de longucur d'une Toife de fer, expofée à différens degrés de chaleur.

75

ART. XXII. Comparaifon de la longueur de la Toife lors de la mefure des deux Bafes. 80

A R T. XXIII. Comparaifon de la mefure actuelle de la Bafe de Tarqui à fa longueur calculée. 85

ART. XXIV. Si toute errenr d'obfervation, qui fera trouver trop long le dernier côté conclu des Tivangles de la Méridienne, doit aulfi néceffairement faire trouver trop longue la Méridienne calculée.

ART. XXV. De combien une différence d'une toife fur la Baje de Tarqui, doit changer la longuenr de la Méridienne.

Ant. XXVI. Autres manières de trouver l'équation de la longueur de la Méridicune, pour une toije de différence fur la Bafe.

95

ArT. XXVII. Détermination de la longueur de l'arc, compris entre les deux Obfervatoires, an Nord \&. au Sud de la Méridieune. 10 I 


\section{SECONDE PARTIE.}

Mefure aftronomique de l'arc du Méridien, ou Détermination de la valeur de l'arc célefle, qui répond à la mefure géométrique.

page 105

Article I. De l'ancien Sectell apporté de France; des changentens qui y furent faits pour le rendre propre alux uouvclles obfervations. $\quad 106$

Article II. Defcriptiou du Secteur.

I IO

Article III. De l'Obferratoire de Tarqui. Déternination de la valeur des parties an Micrometre. Preparatifs communils à toutes nos objervations de l'ciluplitude de l'arc.

Article IV. De l'arc tracéfur le Sectelir. Manière d'obferver la diftance d'une étoile au Zénith fans le fecours des divifions ordinaires. $\quad$ i 6

ARTICLEV. Des différentes obfervations aftronomiques, faites dans la Province de Quito, pour déterniner l'anplitude de l'arc dn Méridiell.

ARTIClE VI. Premières ob fervations à Tarqui, extrémité auftrale de la Méridienme, en Noventbre ơ Décembre 1739 , \& Janvicr 1740.128

Artiche VII. Table d'Obfervations de l'étoile \& dOrion. fuites en communl à Tarqui ell 1739, rée

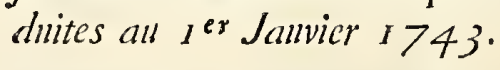

138

Remarques fur les obfervations de la Table. précédenste. 


\section{T A B L E.}

ARdicle VIII. Examen des différentes calles qui peuvent nuire à la jufteffe des obfcrvations.

Des effets du froid or du chaud sur notre Secteur.

I 4 I

Article IX. Suite de l'examen des différentes caufes, $\mho \sigma c$.

De la flexion de l'Inftrument dans le plan du Limbe.

143

A RTICL E X. Continuation du même Jujee.

De la flexion du rayon dans le plan perpendiculaire à celui de l'Inftrument; \& du $P_{a-}$ rallélifine de la Lunctte à ce méme plan. 148

Article XI. Continuation du mếme Jujet.

Do la caufe qui a pî augmenter la diflance apparente de l'itoile au zénith, à Tarqui en 1739 .

152

Article XII. Premières obfervations, faites à Cotcherquï, extrémité feptentrionale de la Méridierne, en Févier, Mars \& Avril 1740 . I 58

A R T. XIII. Table d'Obfervations de l'étoile \& d'Orion, faites en communn à Cotchefqui en 1740 , réduites all I er Janvier $1743^{\circ}: \quad 168$

Remarques. fir les obfervations de la Table. précédente.

169

A R T. XIV. Obfervations diverfes de l'étoile \& d'Orion, faites à Quito, en deux différens endroits, enI $1737, I 740,174 I$ \& $I 74.2$, réainites

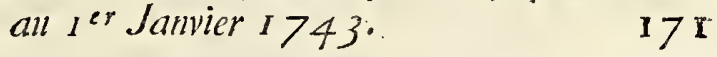

Remarques fur les obfervations de la Table précédente.

A R T. X V. Table des Obfervations de létoile \& d'Orion,

*iji. 


\section{T A B L E.}

faites à Tarqui en 5741 , par $M$. Bou-

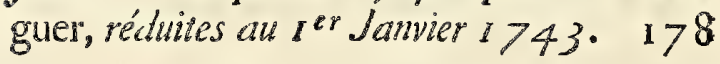

Remarques fur les objervations de la Table précédente.

180

A R. XV I. Dernières obfervations, faitcs à Coichelqui au Nord de la Méridienne, correfpondantes à celles qui ont éré faites en méme temps à l'extrémité Sud.

Table des difances de l'étoile \& d'Orion au Zénith de Cotchefqui, obfervées par $M$. Bouguer à la fin de 1742 , E réduites

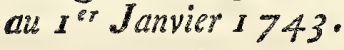

183

Remarques fur les obfervations de la Table précédente.

184

A R T. XVII. Des précautions particuliéres que je pris dans les dernières objervations que je fis à Tarqui en 1742 or 1743 , en correfpondance de celles que $M$. Bouguer faifoit dans le méme temps à l'autre extrémité de la Méridienne.

Secteur raffermi. Sufpenfion perfecionnée. Limbe aplani.

A R T. XVIII. Continuation du même fujet.

Paralléligine de la Lunetze au plan du Secteur. Remarques fur le fil-á-plomb. Mouvement du Secteur dans le plan du Méridien. Inverfions aliernatives de l' Infrument. 19 I

A R T. XIX. Continuation du mếme fujet.

Parallaxe des fils au foyer de la. Lunette, différente pour divers Obfervateurs, \& variable pour le même en différens temps. 196

A R T. XX. Continuation du mêne fujet. 


\section{T A B L E.}

De la manière d'éviter la Parallaxe dis fils au foyer de la Lunette.

A R T. XXI. Dernières obfervations, faites à Tarqui, au Sud de la Méridienne, correfpondantes à celles quii ont été faites en même temps à l'extrémité Nord.

Table des difances de l'étoile \& d'Orion au Zénith de Tarqui, que j'ai obfervées en 1742 \& 1743 , réduites au I ${ }^{\text {er }}$ Jallver 1743 . 2 I $5 \& 216$

Remarques fur les objervations de la Table précédente.

A R T. XXII. Détermination de l'auplitude de l'arc du Méridien, compris entre les Paralleles de Cotchefqui \& de Tarqui, par tontes les oblervations correfpondantes, faites en ces deux lieux en 1742 \& 1743 , \& réduites au premier Janvier $1743^{\circ}$.

A R T. XXIII. Autre détermination de l'amplitude de l'arc du Méridien, compris entre les Paralleles de Cotchefqui \& de Tarqui, par les feules obfervations fimultanées, fans aucune réduttion.

222

OBSERVATIONS SIMULTANEES AUX deUX extrémités de la Méridienne. Amplitude de l'arc célefte, compris entre les deux Ziniths.

A R T. XXIV. Détermination de la longueur du degré du Méridien aux environs de l'E'quateur.

2.27

A R . XXV. De lerreur poffible dans la détermination précédente de la valeur du degré du Méridien. 


\section{T A B L E.}

A r т. XXVI. De linégalité des degrés du Méridien, or de ce qui en réfulte, quant à la figure de la Terre.

ART. XXVII. Des différentes mefures du degré du Méridien, en France. Errenr dans les mefures de $M$. Picard.

Art. XXVIII. Comparaifon de la mefure de l'amplitnde de l'arc du Méridien entre Paris of Amiens, par M. Picard, à celle du même arc, nonvellement mefiré en 5740 .

A r т. XXIX. Examen de la Bafe de M. Picard, o de fa mefine géodéflque de la difance de Paris à Amiens.

ART. XXX. Des divers rapports des axes dn Sphéroüde terreftre, tivés de la comparaifon des divers degrés mefurés.

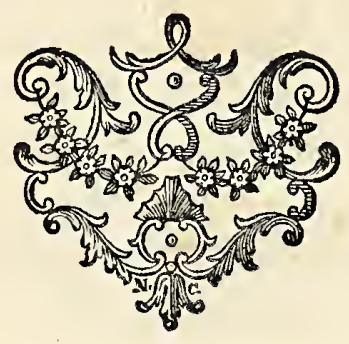


月

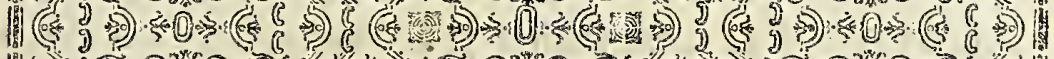

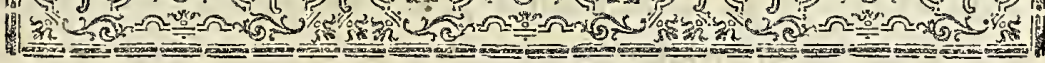

\section{DIVISION DE LOUVRAGE.}

Ce t Ouvrage eft divifé en deux parties. La première contient la Méfure géométrique de trois degrés du Méridien; ou les opérations faites fur le terrein, pour reconnoître la longueur de la Ligne Méridienne que nous avons mefurée dans l'Amérique Méridionale, depuis les environs de Qnito, prefque fous l'Équateur, jufqu'au delà de $C_{\text {luenca, dans l'hé- }}$ mifphère autral.

La feconde partie comprend la Mefure aftronomique de ces mêmes degrés; c'eft-à-dire, les obfervations faites aux deux extrémités de l'arc du Méridien, pour déterminer l'amplitude de cet arc, ou fa valeur en degrés, minutes \& fecondes.

C'eft de mon travail particulier, quant aux opérations trigonométriques, que je rends compte dans la première partie. Le plus grand nombre des obfervations aftronomiques, rapportées dans la feronde, font communes à M. Bouguer \& à moi, ou correfpondantes les unes aux autres.

Nous avons mefuré 3 degrés 7 minutes du Méridien, \& fi les difficultés phyfiques \& morales ne fe fuffent pas trop multipliées, nous euffions prolongé la mefure de cet arc tout le plus loin qu’il nous eût été poffible; perfuadés que c’étoit le moyen le plus fûr de parvenir à une plus exacte détermination du degré.

... Dans une opération de cette nature, on eft expofé à deux fortes d'erreurs : l'une dans la mefure des Triangles qui fervent 
à trouver la longueur de l'arc terreftre; l'autre dans les obfertions aftronomiques, néceffaires pour déterminer l'amplitude de l'arc célefte correfpondant.

Quant à l'erreur aftronomique : on convient qu'elle n'eft pas plus à craindre fur un grand arc que fur un petit ; \& puifque l'erreur totale fe partage entre tous les degrés de l'arc mefuré, il eft clair que plus le nombre en fera grand, moins il y aura d'erreur fur chaque degré. Le plus grand arc eft donc à cet égard le plus avantageux.

Quant à l'erreur géodéfique: il eft vrai quelle peut s'accroître par le nombre multiplié des opérations; mais, I ${ }^{\circ}$ It feroit contre toute vrai-femblance de fuppofer, que les erreurs dans la mefure des angles fur le terrein, fuffent toutes dans le même fens, \& qu'elles s'accumulafient au lieu de fe compenfer, du moins en partie. $2^{\circ}$ Quand on feroit cette étrange fuppoftion, fi l'erreur ne croiffoit que proportionnellement à la longueur de l'arc, elle fe diftribueroit de même fur le nombre des degrés mefurés : elle ne feroit donc pas plus à craindre fur un plus grand nombre de degrés que fur un moindre; \& on ne perdroit encore rien alors de l'avantage de la mefure aftronomique. Enfin fi on vouloit fuppofer que l'erreur géodéfrque crût toûjours du même fens, \& dans une plus grande raifon que le nombre des degrés, ce quion peut regardercomme un cas métaphyfique; les conféquences n'en feroient encore dangereufes qu'autant qu'on n'auroit qu'une feule Bafe mefurée açtuellement: nous ne fommes pas dans ce cas, \& da mefure d'une feconde Bafe fur le terrein, à l'autre extrémité de l'arc, nous met en état d'arrêter le progrès des erreurs qui. pourroient s'être gliffées dans la mefure de nos angles. 


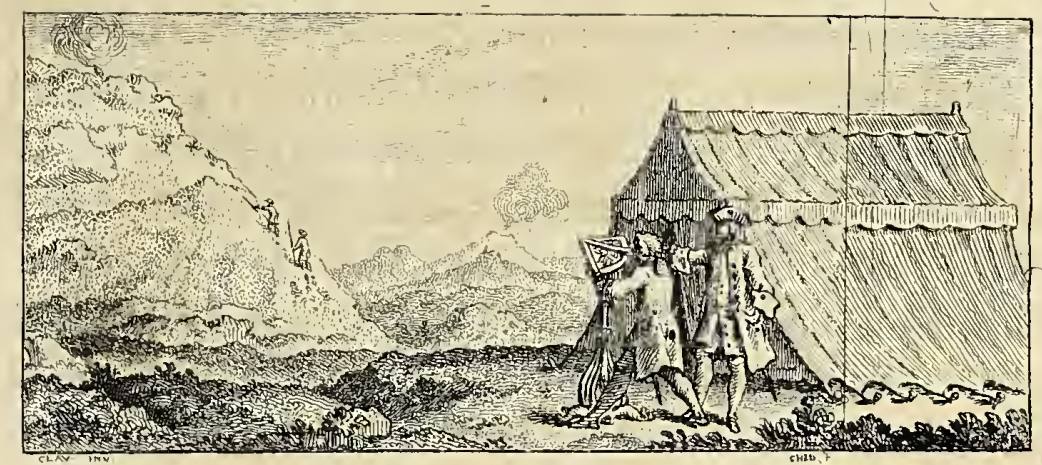

\section{PREMIERE PARTIE.}

MESURE GEOMETRIQUE $D E L^{\prime} A R C D U M E^{\prime} R I D I E N$, O U

\section{OPE'RATIONS SUR LE TERREIN,}

Pour fixer la pofition \&s déterminer la longueur de la Ligne Méridienne.

'Ar cru que le meilleur moyen de préfenter au Lecteur - avec clarté \& précifion le détail d'un grand nombre de différentes opérations, étoit de former une Table qui raffemblant fous un point de vie le plus d’objets quil feroit pofitble; montrât leurs divers rapports \& leur dépendance mutuelle.

C'eft ce que j’ai tâché d'exécuter dans les douze colonnes de la Table de Triangles que je joins ici. J'y ai renfermé les éććmens de la plûpart des opérations que jai faites fur le terrein pour la mefure de la Méridienne, \& les réfultats des 
conféquences quion en peut tirer. On y trouvera les angles de pofition obfervés, corrigés, réduits à l'horizon, la longueur calculée des côtés des triangles inclinés, tels que la nature du terrein nous les a offerts, les angles de hauteur \& de dépreffion réciproquement obfervés entre les Signaux, leurs hauteurs \&. leurs abaiffemens refpectifs en toifes, la longueur des côtés réduits à un même plan horizontal, leurs différentes directions par rapport à la Méridienne, enfin les diftances entre les $\mathrm{Pa}$ rallèles \& les Méridiens des Signaux.

Cette table fait proprement le fond de la première partie de cet ouvrage; les articles qui la fuivent n'en font que le commentaire : ils contiennent l'explication de chaque colonne, \& les fupplémens néceflaires.

Avant que d'entrer dans ce détail, je ne puis me difpenfer' de faire quelques réflexions préliminaires \& quelques remarques fur notre travail, \& fur la manière dont il a été exécuté; je dois commencer par la mefure de la Bafe qui lui fert de. fondement.

\section{A R T I C L E I.}

Mefure actuelle de la première Bafe des Triangles de la Méridienne, aux environs de Quito, dans la plaine d'Yarouqui.

JE ne répéterai point ici le détail d'un Mémoire que j’envoyai en 1736 à l'Académie, fur les précautions que nous avions prifes pour la mefure actuelle de notre première Bafe. Je me contenterai de rappeler que $M$. Godin, qui la mefuroit 


\section{DEGRÉS DU MERIDIEN.}

d'une part, aidé de Don George Juan (a), \& M. Bougutuer \& moi qui la mefurions de l'autre avec Don Antoine de Ulloa \& M. Verguin ( $b)$, nous nous accordâmes à moins de trois pouces près fur une longueur de 6273 toifes.

Dans un pays de montagnes, tel que la province de Quito, le terrein que nous trouvâmes le plus propre à cette opération ne laifloit pas d'avoir une pente de i 26 toifes fur deux lieues, $\&$ cette pente n'étoit pas uniforme. Le parti que nous prîmes, $\&$ le plus convenable en pareil cas, fut de mefurer cette difance horizontalement, en pofant nos perches toûjours de niveau, \& d'obferver la différence de hauteul', tant entre les points intermédiaires \& les plus remarquables de la Bafe, qu'entre fes deux termes extrêmes.

C'eft cette diftance mefurée, pour ainfi dire, par échelons ou gradins à différens niveaux, qu'il faut réduire à la ligne droite. Ce Problème, pris dans toute fa généralité, peut donner lieu à des recherches du reffort de la haute Géométrie: mais l'ćlémentaire fuffit pour le réfoudre avec plus de précifion qu’il n'eft néceffaire dans le cas préfent. Voici les fordemens, le procédé \& le réfultat de mon calcul; j’en fupprime les petits détails, \& jefpère qu'on m’en faura gré.

(a) Don George Juan \& Don Antoine de Ulloa, aujourd'hui Capitaines de vaiffeaux en $E$ fpagne, avoient été nommés par $S$. M. Catholique pour affifter aux obfervations des Académiciens François, \& en tenir regiftre; le fujet de leur miffion eft ainfi exprimé dans les ordres \& paffeports de la Cour d'Efpagne, du 14 Août 1734 , pour notre paffage en l'Amérique Efpagnole: Dos fugetos Efpañoles inteligentes en la Mathematica y Aftroo nomia, para que afiftan, con los inencionados Francefes, a todas las Obfers. yaciones que hizieren, $y$ apunten las que fueren executando.

(b) Ingénieur de la Marine ì Toulon, 
Mefure de la Bafe d'Yarouqui, moyenne entre les deux mefures afuciles prifes horizontalement; ou Somme des petites mefures horizontales à différens niveaux. ................ $6272{ }^{10 i} 7 \%$

De Carabourou, terne feptentrional de la Bafe, hauteur apparente de la mire d'Oyanbaro, terme auftral. $1^{\text {d }} 6^{\prime}$ i $9^{\text {n }}$

D'Oyambaro, abaiffement apparent de la mire du Signal de Carabourou, terme boréal. ...... I. 1 r. 53 .

Différence des deux angles précédens, égale à la valeur apparente de l'arc terreftre compris entre les deux termes de la Bafe.................... 5. 34.

Arc véritable tiré de la valeur du degré, poftérieurement connue, \& répondant à la diftance de 6272 toifes..................... 6. 38

Excès de l'arc véritable fur l'arc apparent, ou fomme des réfractions * qui ont altéré les deux angles obfervés...................... 1. 4 .

Abaiffemens apparens au deffous de l'horizon, des points intermédiaires les plus remarquables de la Bare, obfervés à Oyambaro, terme aufral,

Du point $O$

Le point $A$ à 500 toifes de difance, a paru bas de $2^{\text {d }} 13^{\prime} \quad 45^{\prime \prime}$

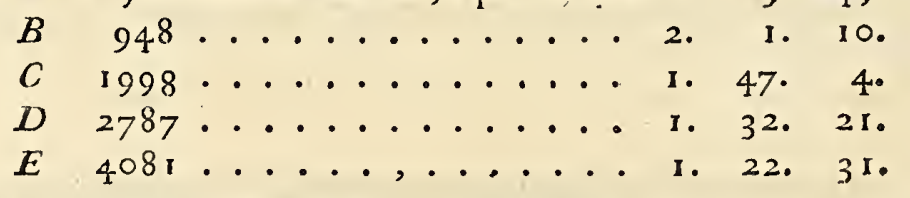

Hautcurs apparentes d'autres points obfervés de

Carabourou, terme autral.

Du point $C$

Le point $F$ à 2093 toifes de diftance, a paru haut de $0.47 . \quad 22 \frac{\mathrm{x}}{2}$ G $400 \ldots \ldots . . . . . . . . .35 .47 \frac{\pi}{2}$

Planche 1, De ces angles de hauteur \& de dépreffion, jai tiré, en Fig. I.

"Voyez ci-apris art. XIII, 
corrigeant la réfraction, la différence de niveau entre tous ces points, \& la hauteur de chacun au deffus du plus bas de tous, qui eft Carabourou, terme boréal de la Bafe.

Hauteur du point $O$ au deffus du niveau de Carabourou. . . $\begin{gathered}\text { toifes. } \\ 26,08\end{gathered}$

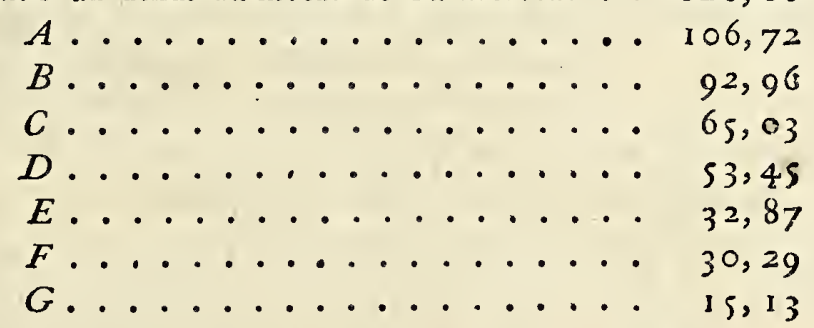

Si la pente du terrein étoit uniforme fur toute fa longueur, chacun des échelons horizontaux de la mefure actuelle fe confondant avec l'arc dont il eft tangente, leur fomme pourroit être réputée égale à l'arc ou à la ligne de niveau, prife à une hauteur moyenne entre les hauteurs des deux termes extrêmes: mais la pente totale du terrein de la Bafe étant inégalement diftribuée fur fa longueur, la fuppofition précédente pourroit s'écarter de la vérité : \& je ne me la fuis pas permife. Cependant cette fuppofition, qui feroit trop peu exacte à l'égard de la longueur totale de la $\mathrm{Bafe}$, ne tire pas à conféquence, quant aux petites portions dans lefquetles je l'ai divifée; chacune d'elles étant comprife entre deux points peu éloignés l'un de l'autre, \& dont la différence de niveau eft connue. Tels font les points défignés dans la lifte précédente; ils ont été choifis comme les plus apparens, \& les feuls où l’on ait pû foupçonner à l'œil quelque changement d'inclinaifon dans le terrein.

C'eft en partant de cette fuppofition \& de celle de la hauteur de Carabourou, que j'établirai en fon lieu de 1226 toifes au 
deffus du niveau de la mer, que j’ai réduit à une même hauteur les mefures des intervalles $O A, A B, B C, C D$, \&c. par leurs différences de niveau.

La Table fuivante donne le réfultat du calcul.

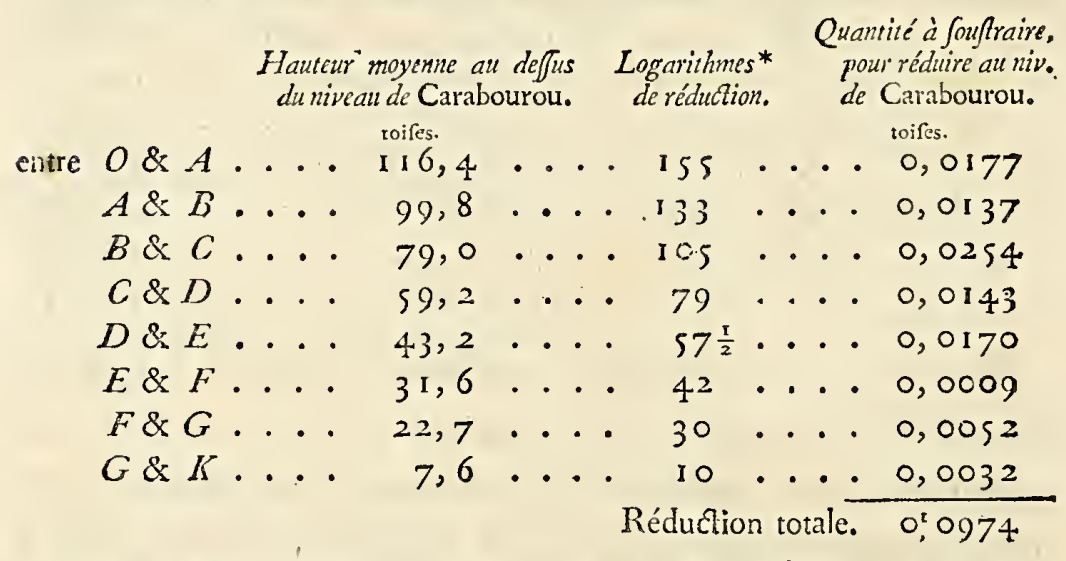

II y a donc 7 pouces I ligne $\frac{1}{2}$, ou o toifes, 0974 à retrancher de 6272 toiles 4 pieds 7 pouces 4 lignes $\frac{1}{2}$, ou de $6272^{t}, 7691$, réputées mefure actuelle de la Bafe, pour Ia réduire à la ligne horizontale, ou à l'arc au niveau de fon point le plus bas, qui eft Carabcurou. Cet arc fera par conféquent de $6272^{t}, 67$ I 7 ; \& en ôtant ot,o I 58 , pour le réduire à fa Planche 1, corde, on aura $6272^{t}, 6559$. Maintenant dans le Triangle fig. $3 \cdot$ $O G K$, comoifint $G K$ corde de l'arc $G F K$, \& les angles $O$. $\& K$ qu'on peut déduire des angles de dépreffion \&s de hauteur,

* J'ajoute pour la hautcur abfoluc de Carabourou I 226 toifes au rayon de Ia Terre, que je fuppofe à l'E'quateur de 3268219 toifes en prenant un milieu entre les hypothères les plus différentes. Le logarichme de ce nombre croît pour 1000 toifes de 0,0001329 . Donc de $0,0000001 \frac{\pi}{3}$ pour chaque toife. Et puifque les différences des arcs font proportionnclles à celles des rayons, on aura la même différence entre le logarithme.de chaque arc mefuré à un certain niveau, \& celui de l'arc correfpondant au niveau de Carabourou. C'eft ainfi qu'on a réduit à ce niveau les arcs $O A, A B, B C, C D, \&$ c. 


\section{DEGRÉS DU MERIDIEN:}

obfervés à Oyambaro \& à Carabourou, on conclurra le côté $O K$, ou la ligne droite inclinée qui mefure $O K$, diftance vraie d'un terme à l'autre de la Bafe : cette diftance fera de $6274^{\mathrm{t}}, 045$, ou de 6274 toifes o pieds 3 pouces 2 lignes $\frac{9}{10}$, c'eft-à-dire, $\mathrm{I}^{\mathrm{t}}, 373$ plus longue que la mefure horizontale au niveau de Carabourou.

Si l'on avoit pris la fomme des girons de marches, ou portions horizontales de la Bafe, au niveau de leur partie fupérieure, on auroit trouvé la mefure horizontale plus longue de 2 pouces 4 lignes $\frac{5}{7}$; j’en ai fait le calcul: \& on l'eût trouvé plus courte de la même quantité, fi l'on avoit fait la fomme en prenant le niveau de la partie inférieure des mêrnes marches." II eft évident que la vraie mefure eft renfermée dans ces étroites limites , \& ne peut différer fenfiblement de leur milieu, auquel je me fuis arrêté.

Comme fur une diftance de 6000 toifes il n'eft pas poffble de répondre de quelques pouces de plus ou de moins dans 1 a mefure actuelle, ce feroit prendre une peine inutile que de porter Ia précifion du calcul fort au delà des bornes prefcrites à notre induftrie. Plufieurs pouces ne font ici d'aucune importance; un pied même, fur une Bafe de 6272 toifes, ne feroit qu'me toife \& un tiers de différence fur le degré, dont on s'eftimeroit heureux d'être affuré à 20 toifes près.

J'aurois pû, par cette raifon, donner la mefure de la Bafe en pouces, fans lignes ni fractions; mais j’ai cru devoir employer les mefures actuelles telles que nous les avons trouvées.

Si jai couru volontairement le rifque de me tromper d'un pouce fur la longueur de la Bafe, ou de 9 pouces fur un degré, j’ai pû négliger fans fcrupule des quantités encore plus 
petites, après m'être affuré par le calcul qu'elles ne montoient quà des fractions de lignes. Telles font les erreurs qui s'enfuivent des fuppofitions que j’ai faites précédemment, en regardant les deux lignes verticales aux deux extrémités de la Bafe, comme concourars̀ à un même point, \& la longueur d'un arc de fix minutes d'un fphérö̈de peu aplati, comme ne différant pas fenfiblement de celle d'un arc pareil dans une fphère du même rayon.

\section{A R T I C L E II.}

Du Syftime de Triangles formé pour mefurer la Méridienne.

Cette Bare de 6000 \& près de 300 toifes, eft le premier côté d'une longue fuite de Triangles plicés à peu près dans la direction du Méridien: cette Suite commence en deçà de la Ligne équinodiale, \& s'étend près de trois clegrés au delà dans I'hómifphère aufral.

Notre principale attention dans l'ordonnance \& la difpofition des Signaux qui devoient terminer nos Triangles, avoit été de choifir, autant que le terrein l'avoit pû permettre, les points propres à rendre les 'Triangles le plus approchans d'être équilatéraux quil avoit été poflble; condition vifiblement la plus avantageufe pour corriger les erreurs qui peuvent fe glifler dans l'obfervation des angles, tant du fait de l'obfervateur que par le défaut des divifions des inftrumens. En eflet, dans toute autre efpéce de triangle que l'équilatéral, il peut y avoir fieu de douter comment doit être répartie entre les trois angles 
fuppolés obfervés avec le même foin, la différence de leur fomme à I 80 degrés; différence que l'on fait être égale à Ia fomme des erreurs commifes dans l'obfervation des trois angles: au lieu quilil eft évident que cette fomme doit être diftribuce également entre trois angles égaux, \& d'autant plus également dans les autres Triangles, qu'ils approchent plus d'être équilatéraux.

Je pourrois ajoûter plufieurs autres remarques \& quelques réflexions fur le même fujet; par exemple, fur les côtés de Triangles fort obliquement oppofés à l'angle qu'ils foutendent, fur les grands côtés conclus par de petits, fur les cas où les angles aigus tirent ou ne tirent pas à conféquence, \&c. Mais ce détail n'eft guère fufceptible d'abrégé; \& je reconnois d'ailleurs que je ne puis mieux faire que d'abandonner à M. Boutguer une matière qu'il fe propole d'approfondir * \& que perfonne ne poffède mieux que lui.

Je me contenterai donc de remarquer àl'égard de nos Triangles, I o Que nous n’avons dans notre fuite directe de Triangles, qu'un feul angle au deffous de 34. degrés parni ceux qui ont fervi à conclurre une diftance; encore en ce cas avons-nous cherché une confirmation par un Triangle auxiliaire.

$2^{\circ}$ Que tous les angles ont été mefurés actuellement.

$3^{\circ}$ Que chaque obfervateur en particulier a mefuré àu moins deux angles de chaque Triangle; \& que le troifième angle, déjà conclu néceffairement par les deux premiers, a cependant encore été vérifié par la mefure actuelle d'un ou de deux autres obfervateurs, pour ne pas laiffer le moindre foupçon d'erreur.

* Voyez le Profpectus de la Figure de la Terre, \&cc. par M. Bouguer, page 3. 


\section{A R T I C LE III.}

Remarques fur les deux différentes fuites de Triangles formées pour la mefure de la Méridienne. Nombre des Obfervaceurs do des inftrumens qui y ont été employés.

T ou t ce'que jai dit jufqüici de notre fyftème de Triangles; convient également aux deux Suites, dont l'une a été mefurée une fois par M. Godin, accompagné de Don George Juan, \& l'autre a été mefurée deux fois, favoir, par M. Bouguer \& Don Antoine de Ulloa, d'une part, \& par moi de l'autre.

Ces deux Suites, compofées chacune de trente \& quelques Triangles, fans y comprendre ceux de vérification ou de confirmation, ont dix-huit Triangles communs (depuis le

Voy. la carte des Triangles de la Méridienne.PI. II.
Triangle IX jufquau XXVI) \& elles ne diffèrent l'une de l'autre, que vers leurs extrémités : la Suite que M. Bouguer \& moi avons mefurée s'étendant plus loin du côté du Sud que celle de M. Godin, \& celle - ci s'avançant vers le Nord plus que la nôtre. Ce que jai dit, que chaque obfervateur a mefuré en particulier deux angles au moins de chaque Triangle, doit s'entendre des Triangles communs aux deux Suites; quant à ceux qui appartiennent à l'une exclufivement à l'autre, tous leurs angles ont été mefurés par l'obfervateur qui a adopté cette Série.

Nous avons donc, je le répète, parce que ceci a befoin 'd'être éclairci, deux Suites de Triangles, lefquelles ne diffèrent que vers leurs extrémités; \& ces deux Suites fourniffent 
trois Mefures trigonométriques différentes, complètes \& indépendantes l'une de l'autre, favoir, la Mefure de M. Godin \&. de Don George Juan, celle de M. Bouguer \& de Don Antoine de Ulloa, \& celle qui m'eft particulière.

Les angles de la première de ces trois Mefures ont été obfervés alternativement avec le Quart-de-cercle de $M^{\text {rs les }}$ Officiers Efpagnols de 24 pouces, \& celui de M. Godin de 2 I pouces de rayon. La lifte de ces angles eft rapportée, (p. I 59 \& fuivo) dans le Recueil d'obfervations, publié à $M a$ drid en $1748^{*}$. La lifte des angles de la feconde mefure, obfervés par M. Bouguer \& par. Don Antoine de Ulloa, avec le Quart-de-cercle de M. Bouguer de $2 \frac{1}{2}$ pieds de rayon, a été pareillement publiée, à quelques angles près, dans le même $\mathrm{Ou}-$ vrage, $p .2$ I 8 \& fuiv. \& vient de l'être par $M$. Bouguer. Je n'en ferai point mention, non plus que de la précédente.

La valeur des degrés. du Méridien que j’établirai ici, fera uniquement tirée, quant à la partie géodéfique, d'une troifième Mefure qui n'a pas encore été publiée : c'eft celle qui m'eft propre.

\section{A R T I C L E IV.}

De ma Mefure géométrique particulière.

JE me fuis fervi pour cette Mefure d'un Quart-de-cercle de trois pieds de rayon, que l'Académie avoit acquis de la fucceffion de feu $M$. le Chevalier de Louville, \& avec Iequel

* Par Don George Juan \& Don Antoine de Ulloa, Capitaines de vaiffeaux de S. M. C. \&c. 
il avoit travaillé à la détermination de l'obliquité de l'E'clip. tique en $172 \mathrm{r}$.

Outre Mrs les Officiers Efpagnols, qui fe font fait un plaifr de feconder $\mathrm{M}^{\mathrm{r}} \mathrm{G}$ Godin \& Bouguer, ceux-ci avoient deux aides intelligens*, aufquels ils auroient pû, au befoin, s'en rapporter pour la mefure de leurs angles. J'ćtois moins heureufement partagé. Dénué d'un pareil fecours, il m’a fallu redoubler d'attention pour prévenir les méprifes \& les erreurs prefque inévitables, dans un fi grand nombre d'opérations; d'autant plus qu'elles ont été exécutées le plus fourvent dans des poftes fort incommodes \& expolés à toutes les injures de l'air. Voici les principales précautions 'que j’ai prifes.

J'ai cru devoir en rendre compte, ou au moins les indiquer; uniquement pour faire voir que je n'ai rien omis ni négligé de ce qui pouvoit de ma part tendre à la perfection de l'ouvrage confié à nos foins; \& non pour infmuer que tous mes angles approchent plus du vrai que ceux des autres Obfer* vateurs, ce que je fuis fort éloigné de prétendre.

I. Il n'y a pas un feul angle de tous ceux que jai obfervés; que je n'aye mefuré plufieurs fois, fouvent en différens jours, $\&$, quand cela a été poffible, à des heuresoù les Signaux fur lefquels on pointoit la lunette, étoient différemment échairés.

$2^{\circ}$ En eftimant les minutes \& leurs fractions fur la divifion du limbe de mon Quart-de-cercle, je ne me fuis jamais fervi des Tranfverfales, mais toûjours du Micromètre, \& de deux manières différentes; c'eft-à-dire, en tourmant la vis alternativement d'un fens \& de l'autre, jai pris un milieu entre les petites différences, quand il s'en eft trouvé.

* Les freurs Hugot Horlogcr, \& Grangier, aujourd'hui Arpenteur royal à Saint-Domingue. 
$3^{\circ} J^{\prime}$ ai rapporté prefque toûjours l'angle que je mefurois à deux différens points de la divifion de l'inftument, diftans I'un de l'autre de dix minutes. La valeur de cet intervalle étoit conmue en parties du Micromètre, \& devoit être égale à la fomme des parties à ajoûter à l'une des mefures, \& à foufraire de l'autre; ce qui fervoit de vérification. •

$4^{\circ} \mathrm{La}$ communication réciproque de la mefure de nos angles obfervés avec différens inftrumens, faite fur le lieu même, \& fouvent auffi-tôt après l'obfervation, étoit encore un bon moyen pour reconnoître \& vériffer fur le champ les équivoques ou erreurs de chiffres.

5० J'ai remarqué que nous nous étions fait une loi de ne conclurre aucun angle, qui n'eût déjà été actuellement mefuré; \& que chacun de nous en particulier a toûjours obfervé au moins deux angles de chaque triangle. J'ajoûte que non feulement dans la melure particulière dont je rends compte, je me fuis conformé à l'arrangement prefcrit, mais que je n'ai omis de mefurer moi-même aucun angle, quand cela s'eft pû faire, faus perdre de temps : \& s'il ne m'a pas été poffible, fans retarder l'ouvrage, de me tranfporter avec mon Quart-decercle à tous les Signaux, il eft du moins certain que dans tout le cours de la Méridienne, il n'y a que trois Signaux où je n'aye pas obfervé; \& que de I 20 angles dont la fuite, qui nous eft commune à M. Bonguer \& à moi, eft compolée, y compris ceux des triangles de confirmation, j’en ai mefuré réellement moi-même I I o avec le même Quart-de-cercle, \& en prenant les précautions que $\ddot{j}$ ai indiquées. Les lettres $a \& b$ diftingueront dans ma Table les dix angles que j’ai employés dans ma lifte, en les tirant de celle de $M^{\mathrm{r} s}$ Godin \& $D$. George, quoique 
16 MESURE DES TROIS PREMIERS

jeuffe pû me contenter de les conclurre de mes feules obfervations. a défigne le Quart-de-cercle de 2 I pouces, $b$ celui de 24 pouces, \& $c$ celui de 30 pouces de rayon.

\section{A R T I C L E V.}

Des différentes corrections faites aux angles obfervés.

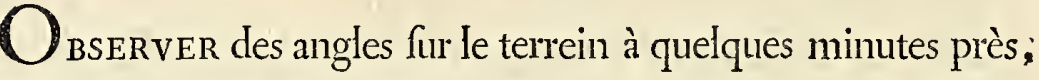
eft une pratique très-fimple $\&$ très - facile: elle eft fuffifante pour les ufages ordinaires. Chercher la précifion dans un angle de pofition, jufquà s'affurer d'une fraction de minute, jufqu'à difcuter quelques fecondes de plus ou de moins, eft une opération fi délicate, quiil n'y a que ceux qui l'ont tentée qui puiffent en bien fentir toutes les difficultés. Dans tous les arts, comme dans une longue \& pénible carrière, les derniers pas que l'or fait font toûjours incomparablement les plus difficiles.

Je fuppofe l'angle auffi exactement obfervé qu'il peut l'être; il y a quelquefois encore quatre \& cinq corrections à y faire, pour en déduire l'angle véritable, ou plûtôt pour en approcher autant qu'il eft poffible.

\section{Premitere Correction.}

\section{Défaut de parallélifme dans la Lunette.}

La vérification de la pofition de la lunette fixe du Quart: de-cercle, pour reconnoitre fi fon axe optique eft en effet; comme on le fuppofe, parallèle au rayon qui paffe par le point $o$, où commence la graduation, \& Ia correction qui en réfulte; font connues \& pratiquées de tous les Obfervateurs. 
Je n’ai jamais manqué de répéter cette correction; non feulement chaque fois que le Quart-de-cercle a été tranfpor'té, ce qui la rend indifpenfable, mais encore, prefque toûjours, à chaque angle que j’ai obfervé, ou au moins au commencement $\&$ à la fin de chaque opération.

\section{SECONDE CORRECTION. \\ Erreur des Divifions.}

La plus difficile \& la plus négligée des corrections, quoique fouvent la plus importante, eft celle qui regarde la vérification des divifions de l'inftument. Mais que n'en coûte-t-il point pour faire cet examen!

Je ne ferai qu'indiquer les principaux moyens que j’ai mis en ufage en différens temps \& en différens lieux, pour reconnoitre les erreurs de la divifion de mon Quart-de-cercle, \& en faire une Table.

Le tour de l'horizon qui doit valoir 360 degrés, en quelque nombre d'angles qu’il foit partagé, \& la fomme des trois angles, qui dans tout Triangle obfervé doit toûjours être égale à I 8 o degrés, font les deux moyens les plus fimples \& les plus ordinairement pratiqués, pour reconnoître fi les divifions font exactes. Je les ai employés utilement. Le premier, dans un pays de montagnes tel que celui où nous étions, exige un calcul long \& pénible. A ces deux moyens, j’en ai joint un troifième de même efpéce, auffr aifé à imaginer que difficile à bien exécuter; \& c'eft celui qui m'a le mieux réuff : mais ce n'eft qu'après trois ans de tentatives peu fatisfaifantes que jai rencontré dans la plaine de Tarqui, près de Cuenca, uu terrein uni tel que l'exigeoit cette opération. 
Je parvins, non fans peine, à pofer en ligne droite, trois points diftans l'un de l'autre d'environ i 5 o o toifes. Je plaçai le centre de mon Quart-de-cercle, difpofé horizontalement, ou pluftôt l'interfection des deux lunettes, fur le point du milieu; \& je divifai le demi-cercle, dont ma ligne droite étoit le diamètre, en deux, en trois, quatre, cinq, fix angles égaux, \&c. par des mires très-diftinctes, pofées à la diftance de 4 à 500 toifes: je vérifiai ainf les angles de $90,60,45,36,30$, I 5 , \&c. degrés, fous-multiples de 180.

J'ai fait d'autres eflais, pour vérifier les divifions de degré en degré : voici celui dont j’ai tiré le plus de parti. A une diftance de soo toifes exactement mefurées, \& prifes pour rayon, je tendis un cordeau à angle droit; je plaçai fur cet alignement des mires, à la longueur calculée des tangentes, de degré en degré. Enfuite dirigeant la lunette immobile du Quart-de-cercle fur la première mire, \& pointant l'alhidade fucceffivement fur les fuivantes, j’examinois fi elle répondoit aux divifions correfpondantes du limbe, ou de combien elle s'en écartoit.

Avec toutes ces méthodes rectifiées l'une par l'autre, \& par la fréquente comparaifon des angles obfervés avec mon Quartde-cercle, aux mêmes angles obfervés avec d'autres inftrumens, dont les divifions avoient auffr été examinées, je fuis parvenu à dreffer une Table des erreurs de mon Quart-de-cercle, de degré en degré.

Auffi-tôt que je l'eus achevée, au mois d'Avril I 740 , je In remis à $M^{\text {rs }}$ Godin \& Bouguer, avec la lifte de tous mes Triangles corrigés conformément à cette Table : ce font les mêmes que je donne aujourd'hui. Il eft vrai qu'en repaffant 
toute cette matière, \& en répétant mes calculs pour la troifième fois, depuis mon retour en France, j’ai été tenté de faire à certains angles quelques corrections, qui euffent mieux fait convenir le calcul de la mefure conclue avec la mefure actuefle de la feconde $\mathrm{Bafe}$; mais jai mieux aimé renoncer à cette apparence d'exactitude, que d'encourir le foupçon de l'avoir cherchée après coup. Je donnerai donc ici mes angles, tels que je les avois corrigés avant que d'avoir calculé les côtés.

\section{TROISI ÈME CORRECTION。}

\section{Réduction au centre.}

Il eft rare quon ait la commodité de placer un Quart-decercle au centre du Signal d'où l'on obferve; \& il eft impoffible d'y en placer deux en même temps. Ainfi de deux Obfervateurs dans le cas dont il eft ici queftion, il y en a au moins un qui opère à quelque diftance du centre. D'ailleurs l'interfection des deux lunettes de linftrument changeant à chaque angle, il faudroit, quand on a plufreurs angles à oblerver dans la même ftation, tranfporter, pour chaque angle, le pied du Quart-de-cercle. Il eft fouvent plus court \& plus commode de remarquer à quelle diftance, de quel côté, \& dans quelle direction l'on eft, à l'égard du centre du Signal. Avec cela; \& la diftance des objets obfervés à peu près connue, on eft en état de réduire l'angle obfervé à celui qui l'eût été du centre même. Cette correction, qui n'eft que de quelques. fecondes quand l'objet eft éloigné \& qu’on n'eft qu'à quelques pieds du centre, exige quelquefois Ia réfolution de deux Triangles. 


\section{QUATRIÈME CORRECTION}

Par la fomme des trois angles.

Si les trois angles d'un Triangle ont été bien obfervés, it eft clair, qu'après les corrections précédentes, leur fomme doit être égale à deux angles droits. Si elle en diffère, \& qu'il n'y ait aucune raifon de rejeter fur un angle pluftôt que fur un autre Ia petite différence qui refte, il n'y a guère d'autre parti à prendre, que de la diftribuer également entre les trois angles; fur-tout quand les angles font à peu près égaux, \& que la différence ne va qu’à quelques fecondes.

\section{Autres Corrections.}

Voilà donc quatre corrections au moins, auxqquelles font -ordinairement fujets tous les angles obfervés; fans parler des corrections extraordinaires, caufées tantốt par quelquaccident étranger à l'Obfervateur : comme la chûte ou l'inclinaifon d'un Signal, ou la diverfe manière dont il étoit éclairé lorfquil a été obfervé; 'tantôt par quelque vice particulier à une obfervation, caufé par une erreur particulière; foit d'un point non vérifié des fubdivifions de dix en dix minutes, foit d'un dérangement paffager dans l'objectif, lequel peut être produit par la dilatation fubite du tuyau de la lunette, expofé par fa partie fupéxieure à un coup de foleil ardent \& peu durable: enfin par mille autres accidens phyfrques, qui naiffent fous les pas des Obfervateur's, \& qui font leur fupplice quand ils afpirent à une grande précifion. 


\section{A R T I C L V VI.}

Table des Triangles de la Méridienne de Quito.

Cetre Table contient, en douze colonnes, la plufpart des conféquences quion peut tirer de nos opérations pour la mefure de la Méridienne, \& la réponfe à la plufpart des queftions qu’on Voy. la $T a-$ ble des Triangles, p. $22 \&$ peut faire fur les différentes parties de ce travail. Voici les titres de chacune des douze colonnes. I. Ordre \& Plans des Triangles. II. Noms des lieux oì étoient pofés les Signaux. III. Angles de pofition obfervés. IV. Équation pour la fomme des trois angles. V. Longueur des côtés oppofés aux angles obfervés. VI. Angles de hauteur \& de dépreffion apparente obfervés, \& Quarts-de-cercle (qui ont fervi aux obfervations). VII. Hauteurs \& Abaiffemens refpectifs des Signaux. VIII. Angles de pofition réduits à l'horizon. IX. Longueur des côtés horizontaux, réduits au niveau de Carabourou. X. Direction des côtés des Triangles par rapport à la Méridienne. X I. Diftances entre les Parallèles des Signaux. XII. Diftances entre les Méridiens des Signaux.

Les articles qui fuiviont la Table, feront employés à en donner l'explication, colonne par colonne : je joindrąi à cette explication le détail de ce qui n’a pû entrer dans la Table même. 


\begin{tabular}{|c|c|c|c|c|c|}
\hline & & \multicolumn{4}{|c|}{ TABLE du Calcul des Triangles } \\
\hline $\begin{array}{c}\text { OrDRE \& PLANS } \\
\text { des } \\
\text { TRIANGLES. }\end{array}$ & $\begin{array}{c}\text { I I. } \\
\text { Noms DES LIÉUX } \\
\text { où étoient pofés } \\
\text { Ies Signaux. }\end{array}$ & \begin{tabular}{|c|c} 
I I I. \\
A NGLES \\
DE POSITION \\
obfervés.
\end{tabular} & $\begin{array}{l}\text { I V. } \\
\text { Equation } \\
\text { pour } \\
\text { Ia toum } \\
\text { des } \\
\text { dAngle. }\end{array}$ & $\begin{array}{c}\text { V. } \\
\text { LONG U E U R } \\
\text { des } \\
\text { côtés oppofés } \\
\text { aux Angles obfervés. }\end{array}$ & $\begin{array}{c}\text { V I. } \\
\text { A N G L E S } \\
\text { de hauteur \& de } \\
\text { dépreffion apparente } \\
\text { obfervés. }\end{array}$ \\
\hline & \begin{tabular}{|l} 
Pamba-marca..... \\
Carabourou......... \\
Terne No.d de la Bare. \\
Oyambaro.......... \\
Terme Sud de la Bare \\
\end{tabular} & $\begin{array}{l}\text { P. } 38^{\circ} 36^{\prime} 14^{\prime \prime} \\
\text { C. } 77 \cdot 35 \cdot 40 \\
\frac{63 \cdot 48.16}{180.0 .10} \\
\end{array}$ & $\begin{array}{l}-3 \\
-4 \\
-3 \\
-10\end{array}$ & $\begin{array}{c}\text { CO. } 6274,05 \\
\text { Bafe inclinéc. } \\
\text { PO. } 9821,00 \\
\text { PC. } 9022,96\end{array}$ & $\left\{\begin{array}{l}\text { C. }-5^{\circ} 41^{\prime} 20^{\prime \prime} \text { calcul. } \\
0 .-4 \cdot 30.27 \cdot \quad c\end{array}\right.$ \\
\hline & $\begin{array}{c}\text { Pamba-marca..... } \\
\text { Oyambaro.......... } \\
\text { Tanlago............. }\end{array}$ & $\begin{array}{l}\text { P. } 69 \cdot 46.37 \\
0.74 \cdot 10.58 \\
\text { T. } 36.2 .25 \\
180 . \quad 0.00 \\
\end{array}$ & $\frac{0}{\circ}$ & $\begin{array}{l}\text { OT. } 15663,0 ; \\
\text { PT. 16060,29 } \\
\text { PO. } 9821,00\end{array}$ & 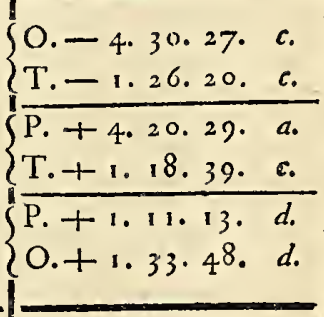 \\
\hline II & $\begin{array}{l}\text { Pamba-marca..... } \\
\text { Tanlagoa........... } \\
\text { Pitchincha.......... }\end{array}$ & $\begin{array}{l}\text { P. } 38.36 .34 \\
\text { Т. } 89.14 .10 \\
\text { П. 52. } 9.22 \\
180.00 .6 \\
\end{array}$ & $\begin{array}{l}-2 \\
-2 \\
-2 \\
-6 \\
\end{array}$ & $\begin{array}{l}\text { ТП. } 12690,77 \\
\text { РП. } 20335,92 \\
\text { РТ. } 16060,29\end{array}$ & $\left\{\begin{array}{l}\text { T. }-1.26 .20 . c . \\
\Pi .+0.9 .53 . c .\end{array}\right.$ \\
\hline & \begin{tabular}{|} 
Pamba-marca.... \\
Pitchincha.......... \\
Schangailli........
\end{tabular} & 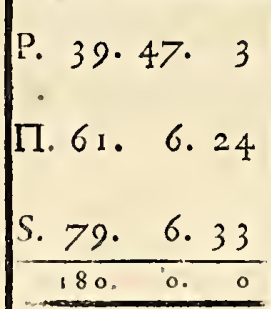 & $\frac{0}{0}$ & $\begin{array}{l}\Pi S .13251,57 \\
\text { PS. } 18131,07 \\
\text { PП. } 20335,92\end{array}$ & 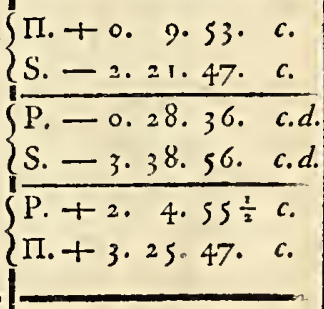 \\
\hline & $\begin{array}{l}\text { Pitchincha......... } \\
\text { Schangailli......... } \\
\text { El Coraf̧on........ }\end{array}$ & $\begin{array}{l}\text { I1. } 58.26 .10 \\
\text { S. } 82.57 .50 \\
\text { C. } 38.36 .12 \\
180.0 .12\end{array}$ & $\begin{array}{l}-4 \\
-4 \\
\end{array}$ & $\begin{array}{l}\text { SC. } 18097,10 \\
\text { ПC. } 21128,15 \\
\Pi S .13251,57\end{array}$ & 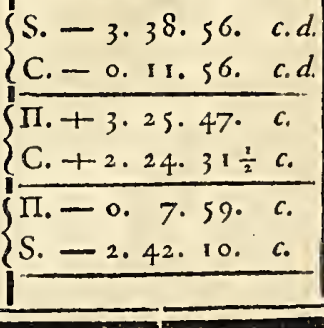 \\
\hline
\end{tabular}




\section{de la Méridienne de Quito.}

V I I.

HaUteIJs

\& Abaiffernens

refpectifs

des Signaux.

Toifes.

C. $-883,85$ ? P. $38^{\circ} 44^{\prime} 42^{\prime \prime}$

0. $-757,64$

P. $+883,85$

\begin{tabular}{l}
$0 .+126,11$ \\
\hline$+1-757,61$
\end{tabular}

P. $+757,64$.

C. $-126,11\}$

C. $77 \cdot 38.28$

$0.63 \cdot 36.50$

O. $-757,64\}$ P. $69 \cdot 49 \cdot 32$

T. $-367,98$

P. $+757,64\}$ O. 74. I 4. 8

T. $+392,82$

P. +367.98
$0 .-392.82$ T. 35.56. 20

$-392,82$

T. $-367,98$ P. $38.34 \cdot 4^{6}$

$\frac{\text { ח. }+113,82}{\text { P. }+367,98}$

II. $+478,07$ T. 89.16.39

P. $-113,82$

T. $-47^{8,07}$

П. 52. 8. 35

$\left.\begin{array}{l}\text { II. }+113,82 \\ \text { S. }-703,20\end{array}\right\}$ P. 39.42 .53

P. $-113,82$ ?

S. $-8,8,06$

P. $+703,20$

ח. $+818,06\}$ S. 79. 12. 33

S. $-818,06$ П. 58.22 .42

\begin{tabular}{l} 
C. $-12,11$ \\
\hline II. $+818,06$
\end{tabular}

C. $+8.07,02$
C. 83 . 5. 22

II. $+12,11$ ?

S. $-807,42\}$ C. 38.31 .56
I X.

LONGUEUR des côtés horizontaux, descôtés des' Triangle réduits au niveau de Carabourou.

Toifes.

CO. $627^{2,66}$ Bafe horizontale réduite.

PO. $\quad 9790,27$

PC. $\quad 8978,38$

OT. 15657,28

PT. 16053,26

PO.

9790,27

ТП. 12679,21

PПI. 20330,69

PT. 16053,26

กIS. 13224,46

PS. $\quad 18115,02$

РПГ. 20330,69

SC. $\quad 18076,76$

ПI C. 21074,38

II S. 13224,46
I $9^{\circ} 25^{\prime} 4^{\prime \prime}$ du Sud à l'Ent objèrvée. Done

44. I I. 46 du Sud à l'Oueft.

82.56 .28 du Sud à l'Oueft.

30. 2. 22 N. $O$.

65.58 .42 N. $O$.

44. I1. 46 S. $\mathrm{O}$.

23. 17.57 S. 0 .

75.26 .32 S. 0 .

65.58 .42 N. $\mathrm{O}$.

43. 28.54 S. E.

35. $43 \cdot 39$ S. $O$.

$75 \cdot 26.32$ S. 0 .

53. 25.44 S. O. \& par obfervation 53. 25. 33

14. 53.48 S. $0 . \&$ par obfervation 14. Si. 44 43. 28.54 S. T:.
X I.

DISTANCE entre

Ies Parallèles

des Signaux.

Toifes.

5915,86

7019,21

I 103,35

$89 \times 0,33$

I 3554,2 I

7837,98

$6,35,00$

14662,94

7019,21

6824,96

11645,24

SI 10,24

$19^{6} 77,97$

6535,00

14662,94

9595,60

9100,04

14705,84

I 0577,92

SIIO, 24

19677,97

10770,50

I $45.17,78$

20366,10

5417,73

9595,60

9100,04 


\section{Suite de la TABLE du Calcul des}

\begin{tabular}{|c|c|c|c|c|c|}
\hline $\begin{array}{r}\text { RDRE \& } \\
\text { des } \\
\text { TRIANG }\end{array}$ & $\begin{array}{c}\text { I I. } \\
\text { NomS DES LIEUX } \\
\text { où étoient pofés } \\
\text { les Signaux. }\end{array}$ & $\begin{array}{c}\text { I I I. } \\
\text { A NGLES } \\
\text { BE POSITION } \\
\text { obfervés. }\end{array}$ & $\begin{array}{l}\text { Equation } \\
\text { pour } \\
\text { la formme } \\
\text { des } \\
3 \text { Angles } \\
\text { Ansuswouss }\end{array}$ & $\begin{array}{c}\text { V. } \\
\text { LONGUEUR } \\
\text { des } \\
\text { côtés oppofés } \\
\text { aux Angles obfervés. }\end{array}$ & $\begin{array}{c}\text { V.I. } \\
\text { A NGLES } \\
\text { de hauteur \& de } \\
\text { dépreffionapparente } \\
\text { obfervés. }\end{array}$ \\
\hline & $\begin{array}{l}\text { Schangailli........ } \\
\text { El Cornson........ } \\
\text { Koto-pacfi......... } \\
\text { Signal de Poued-ouäcou. }\end{array}$ & 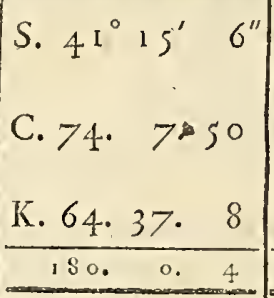 & $\begin{array}{l}-11 \\
-2 \\
-1 \\
-4\end{array}$ & $\begin{array}{l}\text { CK. I } 3207,47 \\
\text { SK. } 19267,09 \\
\text { S C. I } 8097, \text { I o o }\end{array}$ & 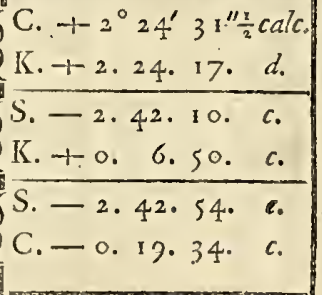 \\
\hline & $\begin{array}{l}\text { El Coraçon........ } \\
\text { Koto-pacfi......... } \\
\text { Papa-ourcou.... }\end{array}$ & 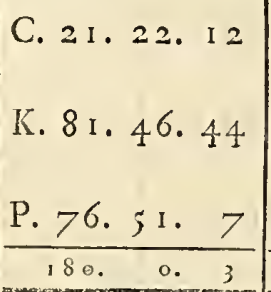 & $\begin{array}{l}-1 \\
-1 \\
-1 \\
-3\end{array}$ & $\begin{array}{l}\text { KP. } 4942,15\} \\
\text { CP. I } 3423,60\{ \\
\text { CK. I } 3207,47\end{array}$ & 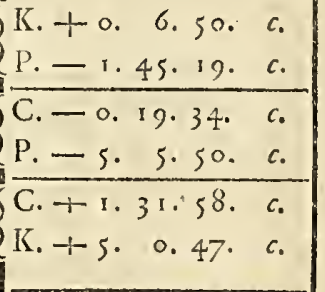 \\
\hline & $\begin{array}{l}\text { El Coraçon........ } \\
\text { Papa-ourcou...... } \\
\text { Milin................. }\end{array}$ & 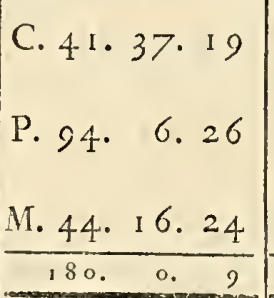 & $\begin{array}{l}-3 \\
-3 \\
-3 \\
-9\end{array}$ & $\begin{array}{l}\text { PM. I } 2772,30 \\
\text { CM. } 19180,17\} \\
\text { CP. } 13423,60\}\end{array}$ & 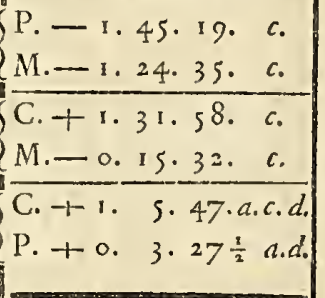 \\
\hline & $\begin{array}{l}\text { El Cornérifier la dift. C........... } \\
\text { Koto-pacfi......... } \\
\text { Milin................ }\end{array}$ & $\begin{array}{l}\text { C. } 62 \cdot 56 \cdot 18 \\
\text { K. } 75 \cdot 17 \cdot 50 \\
\frac{M .41 \cdot 45 \cdot 43}{179 \cdot 59 \cdot 51}\end{array}$ & $\begin{array}{l}+3 \\
+3 \\
+3 \\
+2\end{array}$ & $\begin{array}{l}\text { KM. I } 76,8,09 \\
\text { CM. I } 9180,31 \\
\text { CK. I } 3207,47\end{array}$ & 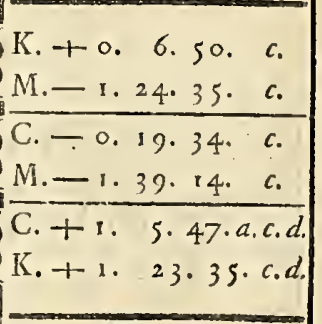 \\
\hline & $\begin{array}{l}\text { Papa-ourcou...... } \\
\text { Milin................ } \\
\text { Ouangotafrn...... }\end{array}$ & $\begin{array}{l}\text { P. 60.31.30 } \\
\text { M. 60.31.54 } \\
\frac{0.58 .56 .36}{150.0 .0}\end{array}$ & 0 & $\begin{array}{l}\text { MO. I } 2979,7^{2} \\
\text { PO. } 12980,58 \\
\text { PM. I } 2772,30\end{array}$ & 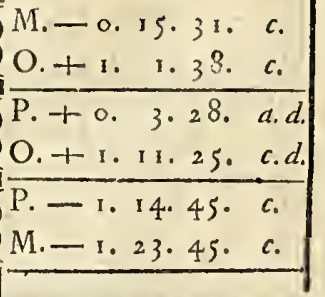 \\
\hline
\end{tabular}




\section{Triangles de la Méridienne de Quito.}

\begin{tabular}{|c|c|c|c|c|c|}
\hline \begin{tabular}{|c|} 
V I I. \\
H A UTEurs \\
\& Abaiffemens \\
refpectifs \\
des Signaux.
\end{tabular} & $\begin{array}{c}\text { V I I I. } \\
\text { A NGLES de pofition } \\
\text { réduits } \\
\text { à l'Horizon. }\end{array}$ & $\begin{array}{c}\text { I X. } \\
\text { L ONG U EU R } \\
\text { des côtés horizontaux, } \\
\text { réduits au } \\
\text { niveau de Carabourou. }\end{array}$ & \begin{tabular}{|c|} 
X. \\
DineCTION \\
des côtés desTriangles \\
par rapport \\
à la Méridienne.
\end{tabular} & $\begin{array}{c}\text { XI. } \\
\text { DIs T A N CE } \\
\text { entre } \\
\text { les Parallèles } \\
\text { des Signaux. }\end{array}$ & $\begin{array}{c}\text { X I I. } \\
\text { Di I T A NCE } \\
\text { entre } \\
\text { les Méridiens } \\
\text { des Signaux. }\end{array}$ \\
\hline $\begin{array}{l}\text { Toifes. } \\
\text { C. }+807,4^{2} \\
\text { K. }+860,53\end{array}$ & $41^{\circ} 17^{\prime} 20^{\prime \prime}$ & CK. $\quad \begin{array}{c}\text { Toires. } \\
3203,95\end{array}$ & $\begin{array}{lll}52^{\circ} & 27^{\prime} & 53^{\prime \prime} \\
\text { du Sud à l'Eft. }\end{array}$ & $\begin{array}{c}\text { Toires. } \\
8044,50\end{array}$ & $\begin{array}{c}\text { Toifes. } \\
\text { I } 0470,45\end{array}$ \\
\hline $\begin{array}{l}\text { S. }-807,4^{2} \\
\text { K. }+50,7^{1} \\
\end{array}$ & $74 . \quad 6.23$ & $S K . .39245,40$ & $\begin{array}{lll}12 . & 8 . & 24 \\
\text { du Sud à l'Oueft. }\end{array}$ & I 88 I 5,00 & 4047,33 \\
\hline $\begin{array}{l}\text { S. }-860,53 \\
\text { C. }-50,7^{1}\end{array}$ & 64.36 .17 & SC. $\quad 18076,76$ & $\begin{array}{l}53 \cdot 25 \cdot 44 \\
\text { du Sud à l'Oueft. }\end{array}$ & 10770,50 & $14517,7^{8}$ \\
\hline $\begin{array}{l}\text { IK. }+50,71 \\
\text { P. }-38,5,12 \\
\end{array}$ & $17 \cdot 28$ & KP. $\quad 4922,82$ & $\begin{array}{c}45 \cdot 45 \cdot 36 \\
\text { s. } 0 .\end{array}$ & 3433,82 & 3526,15 \\
\hline $\begin{array}{r}\text { C. } 50,71 \\
\text { P. }-435,47 \\
\end{array}$ & K. $81 \cdot 46 \cdot 31$ & CP. 3415,48 & $\begin{array}{l}\text { 31. IO. } 25 \\
\text { S. E. }\end{array}$ & $1447^{8,32}$ & 6944,30 \\
\hline $\begin{array}{l}\text { C: }+385,12 \\
\text { K. }+435,47\end{array}$ & 76.56 & CK. 13203,95 & $\begin{array}{l}\text { 52. } 27.53 \\
\text { S. E. }\end{array}$ & 8044,50 & 10470,45 \\
\hline $\begin{array}{l}P:-385,12 \\
M .-419,53 \\
\end{array}$ & I I & PM. 32770,50 & 54. $43 \cdot 34$ & 7374.78 & 10425,85 \\
\hline $\begin{array}{l}\text { C. }+385,12 \\
\text { M. } \quad 35,29 \\
\end{array}$ & . 94.6. & C M. $\quad 9^{\prime} 17^{1,87}$ & $\mid \begin{array}{ccc}\text { 10. } & 27 . & 46 \\
\text { S. O. \& par obferv. réd. } \\
\text { 1 o. } 26 .\end{array}$ & 18853,11 & 3481,55 \\
\hline $\begin{array}{l}\text { C. }+419,53 \\
\text { P. }+35,29\end{array}$ & M. $44 \cdot$ I $5 \cdot 48$ & CP. $13415 ; 48$ & $\begin{array}{l}31 . \text { I O. } 25 \\
\text { S. E. }\end{array}$ & 1478,32 & 6944,30 \\
\hline$\left\{\begin{array}{r}\text { K. } 50 ; 71 \\
\text { M. }+10,53 \\
\end{array}\right.$ & C. 62.155 .37 & KM. $\quad 1>^{6} 48,94$ & $\begin{array}{l}\text { 52. 14. } \\
\text { S. O. }\end{array}$ & 10808,85 & 13951,86 \\
\hline $\begin{array}{l}C .-50,71 \\
M .-469,48\end{array}$ & $\{\mathrm{~K} \cdot 75 \cdot 18.4$ & CM. $1917^{2,10}$ & $\begin{array}{l}\text { I0. } 27 \cdot 44 \\
\text { S. } 0 .\end{array}$ & 18853,36 & $34^{8} \mathrm{I}, \frac{4}{2} \mathrm{I}$ \\
\hline $\begin{array}{l}\text { C. }+419,53 \\
K+469,48\end{array}$ & $41 \cdot 46 \cdot 19$ & CK. $\quad 13203,95$ & $\begin{array}{l}52.27 \cdot 53 \\
\text { S. E. } \\
\end{array}$ & 8044,50 & 10470,45 \\
\hline $\begin{array}{l}\text { M. } 35,29 \\
0 .+257,47 \\
\end{array}$ & P. 60.30 .50 & MO. 12974,21 & $\begin{array}{l}\text { 64. } 44 \cdot 52 \\
\text { S. E. }\end{array}$ & 5534,85 & 11734,37 \\
\hline $\begin{array}{l}\text { P. }+35,29 \\
\text { O. }+292,90 \\
\end{array}$ & M.60.31.3.4 & PO. $\quad 12975,77$ & $\begin{array}{l}\text { 5. } 47.16 \\
\text { S. E. }\end{array}$ & 12909,63 & I30'8,53 \\
\hline $\begin{array}{l}\text { P. }-257,47 \\
\text { M. }-292,90 \\
\end{array}$ & $0.58 .57 \cdot 36$ & PM. $\quad 1277^{\circ}, 50$ & $\begin{array}{l}54 \cdot 43 \cdot 34 \\
\text { s. } 0 .\end{array}$ & $7374,7^{8}$ & 10425,85 \\
\hline
\end{tabular}




\section{Suite de la TA BLE du Calcul des}

\begin{tabular}{|c|c|c|c|c|c|}
\hline $\begin{array}{c}\text { T. } \\
\text { ORDRE \& PLANS } \\
\text { des } \\
\text { TRIANGLES. }\end{array}$ & $\mid \begin{array}{c}\text { I I. } \\
\text { Noms } \\
\text { où Li étoient pofés } \\
\text { les Signaux. }\end{array}$ & $\begin{array}{c}\text { I II. } \\
\text { A NGLES } \\
\text { DE POSITION } \\
\text { obfervés. }\end{array}$ & \begin{tabular}{|c|} 
I V. \\
Equation \\
pour \\
Ia lomme \\
des \\
3 Angles
\end{tabular} & $\begin{array}{c}\text { V. } \\
\text { LONGUEUR } \\
\text { des } \\
\text { côtés oppofés } \\
\text { aux Angles obfervés. }\end{array}$ & \begin{tabular}{|c|c|} 
V I. \\
A N G L S \\
de hauteur \& de \\
dépreffion apparente \\
obfervés.
\end{tabular} \\
\hline & $\begin{array}{l}\text { Milin................ } \\
\text { Ouango-taffin.... } \\
\text { Tchoulapou ...... }\end{array}$ & 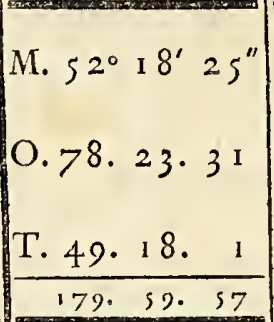 & $\begin{array}{c}+1 \\
+1 \\
+1 \\
+3\end{array}$ & $\begin{array}{c}\text { Toifes. } \\
\text { OT. I } 3547,44 \\
\text { MT. } 16770,32 \\
\text { MO. } 12979,72\end{array}$ & 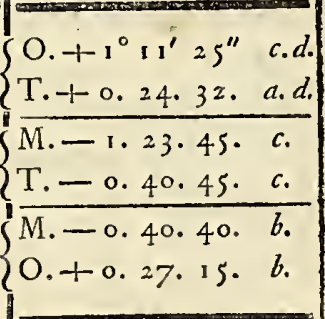 \\
\hline & $\begin{array}{l}\text { Ouango-taffin.... } \\
\text { Tchoulapou...... } \\
\text { Hivicat fou......... }\end{array}$ & $\begin{array}{l}\text { O. 34.47.51 } \\
\text { T. } 73 \cdot 54 \cdot 8 \\
\frac{171 \cdot 17 \cdot 486}{179 \cdot 59 \cdot 47}\end{array}$ & $\begin{array}{l}+4 \\
+5 \\
+4 \\
+13 \\
\end{array}$ & $\begin{array}{l}\text { TH. } 8162,43 \\
\text { OH. } 13741,93 \\
\text { OT. } 13547,44\end{array}$ & 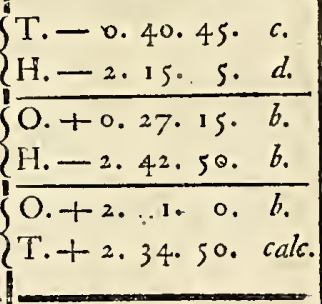 \\
\hline & $\begin{array}{l}\text { Tchoulapou....... } \\
\text { Hivicatfou......... } \\
\text { Chitchitchoco.... }\end{array}$ & 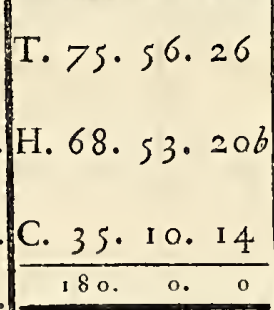 & $\frac{0}{0}$ & $\begin{array}{l}\text { HC. } 13746,66 \\
\text { TC. } 13219,50 \\
\text { TH. } 8162,43\end{array}$ & 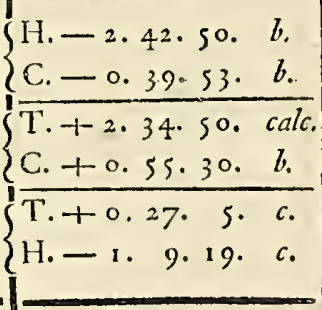 \\
\hline & $\begin{array}{l}\text { Hivicatfou......... } \\
\text { Chitchitchoco... } \\
\text { Moulmoul........ }\end{array}$ & $\left\{\begin{array}{l}\text { H. } 34 \cdot 29 \cdot 33^{b} \\
\text { C. } 72 \cdot 6 \cdot 5 \\
\begin{array}{l}\text { M. } 73 \cdot 24 \cdot 28 \\
80 .\end{array} .6\end{array}\right.$ & $\begin{array}{l}-2 \\
-2 \\
-2 \\
-6\end{array}$ & $\begin{array}{l}\text { CM. } 8122,51 \\
\text { HM. I } 3649,13 \\
\text { HC. I } 3746,66\end{array}$ & 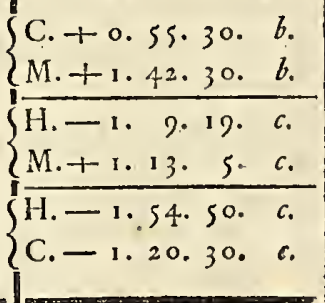 \\
\hline & $\begin{array}{l}\text { Chitchitchoco.... } \\
\text { Moulmoul......... } \\
\text { Ygoalata............ } \\
\begin{array}{c}\text { ou Goayama. } \\
\text { Yon }\end{array}\end{array}$ & $\begin{array}{l}\text { C. } 48 \cdot 51 \cdot 20 \\
\text { M. 54.19.20 } \\
\frac{Y \cdot 76 \cdot 49 \cdot 17}{179 \cdot 59 \cdot 57}\end{array}$ & $\begin{array}{l}+1 \\
+1 \\
+1 \\
+3\end{array}$ & $\begin{array}{l}\text { MY. } 6282,14 \\
\text { CY. } 6776,47 \\
\text { CM. } 8122,51\end{array}$ & 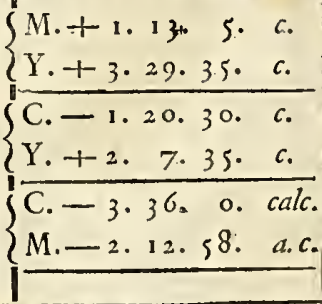 \\
\hline
\end{tabular}




\section{Triangles de la Méridienne de Quito.}

V I I.

HAUTEIRS

\& Abaiffemens refpectifs des Signaux.

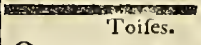

O. $\left.+29^{2,90}\right\}$ M. $52^{\circ}{ }^{\prime} 8^{\prime} 26^{\prime \prime}$ $\frac{\mathrm{T}_{1}+159,03}{\mathrm{M} .-292,90}$

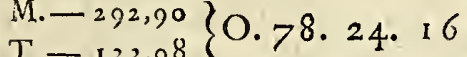
T. -133.98$\}$

$\left.\begin{array}{l}\text { M. }-159,03 \\ 0 .+133,98\end{array}\right\}$ T. $49 \cdot 17 \cdot 18$

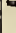

T. - 13.3.98 $\{$ O. 34.46 .33

H. $\left.-511.7^{1}\right\}$

O: $-1-133,98\}$ T. $73 \cdot 51 \cdot 44$

H. $-376,99$

O. $+511,7$ I $\{$ H. 7 I 2 I. 43

T. $\left.+37^{6,99}\right\}$

H. $\left.-37^{6,99}\right\}$ T. $75 \cdot 57 \cdot 21$

C. $-128,75$

T. $\left.+37^{6,99}\right\}$ H. 68. 54.30

C. $+249,54$

T. $+128,75$ ?

H. $-249,54$

C. $+24.9,54\}$ H. $34 \cdot 29 \cdot 34$ M. $+43 \mathrm{r}, 46$

H. $-249,54\}$ C. 72 . 4. 3 M. $+181,4,2\}$

$\left.\begin{array}{l}\text { H. }-431,4^{6} \\ \text { C. }-181,4^{2}\end{array}\right\}$ M. $73 \cdot 26.23$

M. $+181,42$

$\mathrm{Y} .+419,19$

$\left.\begin{array}{l}\text { C. }-181,42 \\ Y .+238,01\end{array}\right\}$ M. 54.13.18

$\mathrm{Y} .+238,01\}$

C. $-419,19$ ?

M. $-238,01\}$
I X.

LONGUEUR des côtés horizontaux, descôtés des Triangles réduits au niveau de Carabourou. Toires.

OT. $\$ 3544,16$

MT. I6767,00

MO. I 2974,2 I

TH. 8152,70

OH. $1373^{\circ, 55}$

OT. 13544,16

H C. 13742,45

TC. 13216,8 ;

TH. 8152,70

CM. 8 I 9,16

HM. I 3640,65

H C. I $37,42,45$

MY. $\quad 6276,27$

CY. $\quad 6762,18$

CM. 8119,16

\section{$\mathrm{X}$.}

DIRECTION

par rapport

à la Méridienne.

a

$36^{\circ} 50^{\prime} 52^{\prime \prime}$ dus Sad à l'Oueft.

I2. 26.26

du Sud à l'En

64. 44. 52

du Sud a l'Eet.

69. $17 \cdot 24$ S. $\mathrm{E}$.

2. 4. 19

s. $\mathrm{O}$.

36.50 .52 s. 0 .

41. 48.6 s. 0 . s. $\mathrm{O}$,

69. $17 \cdot 24$ S. E.

66. 7.5 I S. E.

7. 18.32 s. 0 .

41. 48.6 s. 0 .

59. $38.5 \mathrm{I}$ s. 0 .

17. I6. 5I

SE. \& par obferv, réd.

17. 17.59

66. 7.51
6. 39.57
$\mathrm{X}$ I.

DISTANCE entre

les Parallèles

des Signaux.

Taifes.
I.08 88,47

16373,31

5534,85

I 1734,37

2883 , II

7625,90

I $37^{21}, 57$

496,42

$108,8,47$

8 I 22, 3 I

I 0244,40

9160,09

13127,51

× 534,19

2883,11

7625,20

3285,40

7424,74

I 3529,8 I

1735,34

10244,40

9160,09

3171,52

5416,00

6456,93

2008,74

328,40

7424,74 


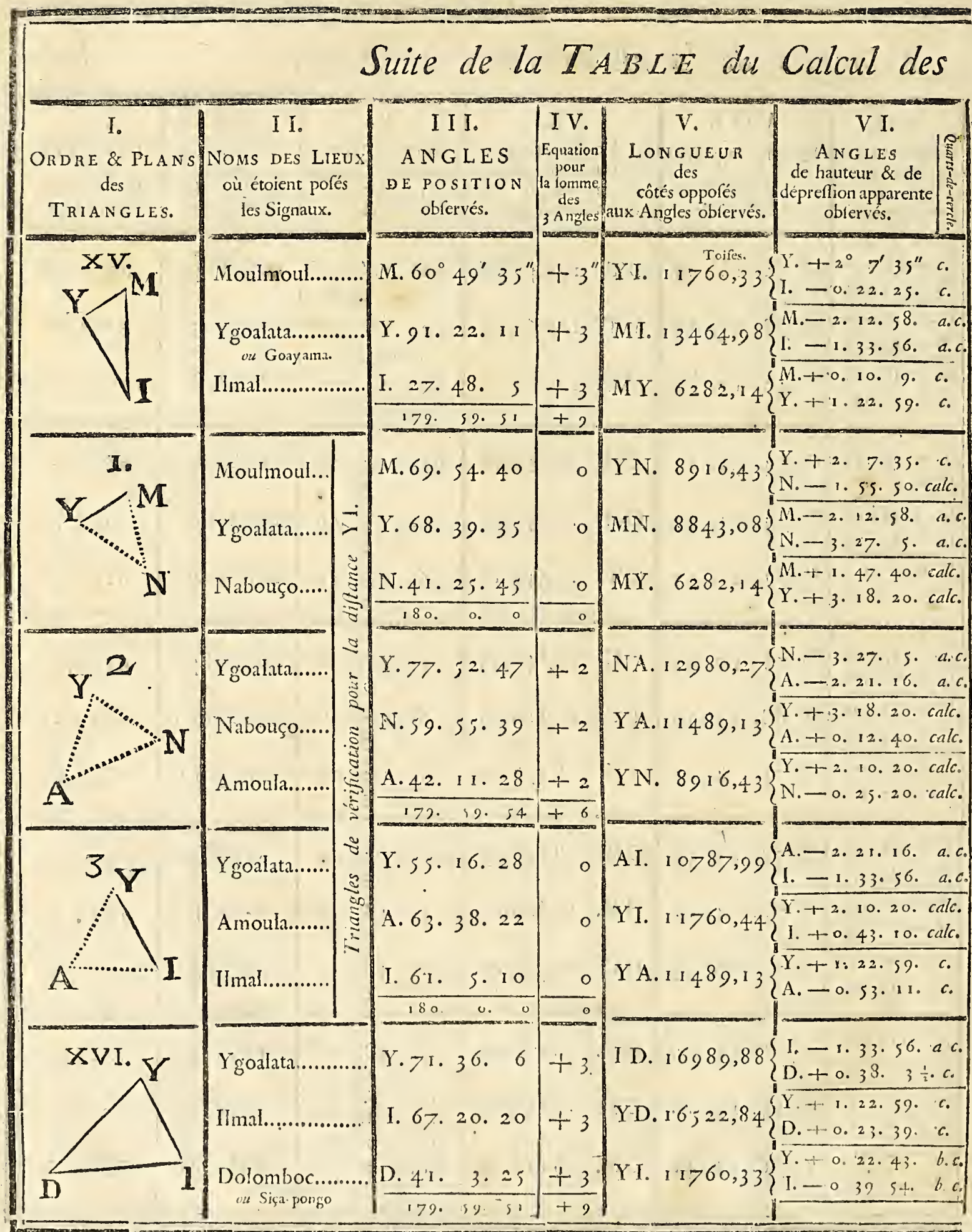




\section{Triangles de la Méridienne de Quito.}

\begin{tabular}{|c|c|c|c|c|c|}
\hline \begin{tabular}{|c|} 
V I I. \\
H A A UTEurs \\
\& A baiffemens \\
refpectifs \\
des Signaux.
\end{tabular} & $\begin{array}{c}\text { V I I I. } \\
\text { ANGLES de pofition } \\
\text { réduits } \\
\text { à l'Horizon. }\end{array}$ & $\begin{array}{c}\text { IX. } \\
\text { L o N G U EU R } \\
\text { des côtés horizontaux, } \\
\text { réduits au } \\
\text { niveau de Carabourou. }\end{array}$ & \begin{tabular}{|c|} 
X. \\
DIRECTION \\
des côtés desTriangles \\
par rapport \\
à la Méridienne.
\end{tabular} & $\begin{array}{c}\text { X I. } \\
\text { Dis T A NCE } \\
\text { entre } \\
\text { les Parallèles } \\
\text { des Signaux. }\end{array}$ & $\begin{array}{c}\text { X I I. } \\
\text { D Is T A N C E } \\
\text { entre } \\
\text { les Méridiens } \\
\text { des Signaux. }\end{array}$ \\
\hline $\begin{array}{l}\text { Toifes. } \\
Y .+238,01 \\
\text { I. }-\quad 63,78 \\
\end{array}$ & M. $60^{\circ} 47^{\prime} 19^{\prime \prime}$ & YI. $\quad \begin{array}{c}\text { Toifes. } \\
\text { I } 754,02\end{array}$ & $\begin{array}{l}28^{\circ} 55^{\prime} \text { I } 2^{\prime \prime} \\
\text { du Sud à l'Eft. }\end{array}$ & $\begin{array}{c}\text { Toifes. } \\
\mathrm{I} O 288,25\end{array}$ & 5684, Io 10 \\
\hline $\begin{array}{l}\text { M. }-238,01 \\
1 .-302,52\end{array}$ & $Y .91 .25 \cdot 57$ & MI. I 3462,44 & S. E. \& par 2 obfer. réd. & 13459,76 & 268,10 \\
\hline $\begin{array}{l}\text { M. }+63,7^{8} \\
\text { Y. }+302,52\end{array}$ & $27 \cdot 46 \cdot 44$ & MY. $\quad 6276,27$ & $\begin{array}{rrr}1 . & 9 \cdot & 27 \\
59 . & 38 . & 5 \\
\text { I }\end{array}$ & 3171,52 & 5416,00 \\
\hline $\begin{array}{l}\mathrm{Y}+2{ }_{2} 8,01 \\
\mathrm{~N}_{2}-{ }_{2} 87,45 \\
\end{array}$ & $69 \cdot 48 \cdot 30$ & YN. 8899,31 & $\begin{array}{l}\text { 51. } 36.6 .24 \\
\text { S. E. }\end{array}$ & 5526,97 & $6,74,97$ \\
\hline $\begin{array}{l}\text { M. }-238,01 \\
\text { N.- } 25,46 \\
\end{array}$ & $68.44 \cdot 45$ & MN. $\quad 8837,09$ & $\begin{array}{l}\text { I0. } \\
\text { S. E. } 39\end{array}$ & $86,8,49$ & 1558,97 \\
\hline $\begin{array}{l}\text { M. }+287,45 \\
\text { Y. }+525,46\end{array}$ & $N .41 .26 .45$ & MY. $\quad 6276,27$ & $\begin{array}{r}59.38 .5 \mathrm{I} \\
\text { s. } 0 . \\
\end{array}$ & 3171,52 & 5416,00 \\
\hline $\begin{array}{l}\text { N. }-525,46 \\
\text { A. }-453.73 \\
\end{array}$ & $77 \cdot 59 \cdot 34$ & NA. 12978,55 & $\begin{array}{l}\text { 68. } 30.24 \\
\text { s. o. }\end{array}$ & 4755,25 & 12076,01 \\
\hline $\left.\begin{array}{l}Y .+525,46 \\
\text { A. }+71,73\end{array}\right\}$ & N. 59.53 .12 & Y A. I I 478,00 & $\begin{array}{l}\text { 26. } 23.10 \\
\text { s. } 0 .\end{array}$ & 10282,21 & 5101,03 \\
\hline $\left.\begin{array}{l}\text { Y. }+453,73 \\
\text { N. }-71,73\end{array}\right\}$ & A. $42.7 \cdot 14$ & YN. $\quad 8899,31$ & $\begin{array}{c}\text { S1. 36. } 24 \\
\text { S. E. }\end{array}$ & 5526,97 & 6974,97 \\
\hline $\begin{array}{l}\text { A. }-453,73 \\
\text { I. }-302,52 \\
\end{array}$ & Y. 55. I 8.15 & A I. I $\quad>84,8$ I & $\begin{array}{l}89 . \text { s8. } \\
\text { s. E. }\end{array}$ & 6,27 & I 0784,8 I \\
\hline $\left.\begin{array}{l}Y .+453,73 \\
\text { I. }+151,16\end{array}\right\}$ & A. $63 \cdot 38.50$ & YI. I I 754,07 & $\begin{array}{l}\text { 28. 55. } 5 \\
\text { S. E. }\end{array}$ & 10288,49 & 5683,78 \\
\hline $\begin{array}{l}\text { Y. }+302,52 \\
\text { A. }-151,16\end{array}$ & I. 61.2 .55 & Y A. I I 478,00 & $\begin{array}{l}26.23 .10 \\
\text { s. } 0 .\end{array}$ & 10282,21 & 5101,03 \\
\hline $\begin{array}{l}\text { I. }-302,52 \\
\text { D. - } 46,01\end{array}$ & Y.71. 36 & I D. $\quad 1698,94$ & $\begin{array}{l}83 \cdot 44 \cdot \text { IS } \\
\text { s. O. }\end{array}$ & $18 ; 2,89$ & 16884,57 \\
\hline $\begin{array}{l}\text { Y. }+302,52 \\
\text { D. }+157,00 \\
\end{array}$ & $\{1.67 \cdot 20 \cdot 33$ & YD. I 6518,42 & $\begin{array}{c}42 \cdot 41 \cdot 32 \\
\text { s. } 0 .\end{array}$ & $1214 x, 14$ & 11200,46 \\
\hline $\begin{array}{l}\text { Y. }+146,01 \\
\text { I. }-157,00 \\
\end{array}$ & D. 41.2 .43 & YI. $\quad$ II 754,02 & $\begin{array}{l}\text { 28. 55. } 12 \\
\text { S. E. }\end{array}$ & 10288,25 & 5684,10 \\
\hline
\end{tabular}




\section{Suite de la TABLE du Calcul des}

\begin{tabular}{|c|c|c|c|c|c|}
\hline $\begin{array}{l}\text { RDRE \& PLANS } \\
\text { des } \\
\text { TRIANGLES. }\end{array}$ & $\begin{array}{l}\text { NOMS DES LIÉUX } \\
\text { où étoient pofés } \\
\text { les Signaux. }\end{array}$ & $\begin{array}{c}\text { I I I. } \\
\text { A NGLES } \\
\text { DE POSITION } \\
\text { obfervés. }\end{array}$ & \begin{tabular}{|l} 
I V. \\
Equation \\
pour \\
ta forme \\
des \\
3 Angies \\
ansen
\end{tabular} & $\begin{array}{c}\text { V. } \\
\text { LONGUEUR } \\
\text { des } \\
\text { côtés oppolés } \\
\text { aux Angles obicrvés. }\end{array}$ & $\begin{array}{c}\text { V I. } \\
\text { A N G LES } \\
\text { de hauteur \& de } \\
\text { dépreffion apparente } \\
\text { obfervés. }\end{array}$ \\
\hline E & $\left\{\begin{array}{c}\text { Pour vérif. la dift. Y. D } \\
\text { Ygoalata.......... } \\
\text { Nabouço........... } \\
\text { Dolomboc........ } \\
\text { ou Siça-pongo. }\end{array}\right.$ & $\begin{array}{l}\text { Y. } 94^{\circ} 15^{\circ} 4^{\prime \prime} \\
\text { N. } 58.23 \cdot 7 \\
\text { D. } 27 \cdot 21.53 \\
180.0 .4\end{array}$ & $\begin{array}{l}-2^{\prime \prime} \\
-1 \\
-1 \\
-4 \\
\end{array}$ & $\begin{array}{l}\text { Toifes. } \\
\text { ND. } 19345,00 \\
\text { YD. I } 6519,44 \\
\text { YN. } 8916,43\end{array}$ & 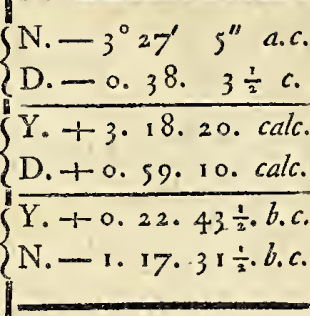 \\
\hline & $\begin{array}{l}\text { Ilmal .............. } \\
\text { Dolomboc...... } \\
\text { Zagroum ....... }\end{array}$ & $\begin{array}{l}\text { I. } 63 \cdot 39 \cdot 35 \\
\text { D. } 48 \cdot 31.31 \\
\text { Z.67.48.57 } \\
180.0 .3\end{array}$ & $\begin{array}{l}-1 \\
-1 \\
-1 \\
-3 \\
\end{array}$ & $\begin{array}{l}\text { DZ. I } 6443,09 \\
\text { IZ. } 13747,24 \\
\text { ID. } 16989,88\end{array}$ & 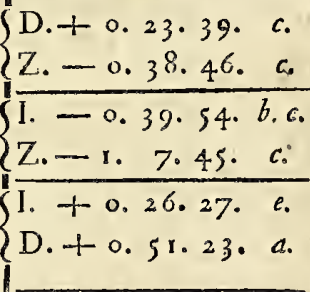 \\
\hline & $\begin{array}{l}\text { Dolomboc......... } \\
\text { Zagroum........... } \\
\text { Lalangouço....... }\end{array}$ & $\begin{array}{l}\text { D. } 47.28 .12 \\
\text { Z. } 52.1 .5 \\
\text { L. } 80.30 .34 \\
179.59 .51 \\
\end{array}$ & $\begin{array}{l}+3 \\
+3 \\
+3 \\
+9 \\
\end{array}$ & $\begin{array}{l}\text { ZL. I } 2285,58 \\
\text { DL. } 13140,49 \\
\text { DZ. } 16443,09\end{array}$ & 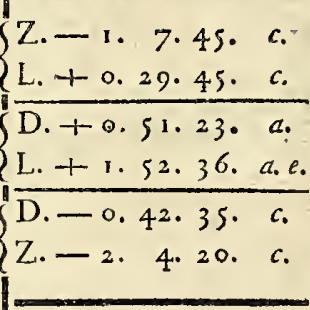 \\
\hline & $\begin{array}{l}\text { Zagroum ....... } \\
\text { Lalangouço.... } \\
\text { Senegoalap..... }\end{array}$ & $\begin{array}{l}\text { Z.71. 1. } 0 \\
\text { L. } 47 \cdot 46.35 \\
\text { S. 61.12.316 } \\
180.0 .6\end{array}$ & $\begin{array}{l}-2 \\
-2 \\
-2 \\
-6\end{array}$ & $\begin{array}{l}\text { LS. } 13256,17 \\
\text { ZS. } 10381,11 \\
\text { ZL. } 12285,58\end{array}$ & 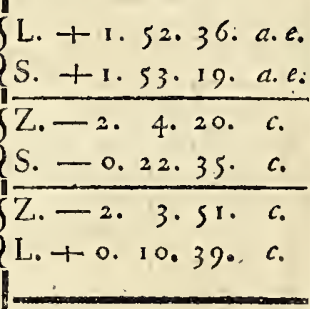 \\
\hline & $\begin{array}{l}\text { Lalangouço....... } \\
\text { Senegoalap........ }\end{array}$ & $\begin{array}{l}\text { L. } 66.28 .33 \\
\text { S. } 55 \cdot 40.506 c \\
\frac{\text { C. } 57 \cdot 50.28}{179.59 .51}\end{array}$ & $\begin{array}{l}+3 \\
+3 \\
+3 \\
+9\end{array}$ & $\begin{array}{l}\text { SC. } 14357,19 \\
\text { LC. } 12932,54 \\
\text { LS. } 13256,17\{\end{array}$ & $\left\{\begin{array}{l}S .-0.22 .35 \cdot c_{0} \\
\text { C. }-1.20 .5 . c_{0} \\
\text { L. +0.10.39. c. } \\
\text { C. }-0.58 .31 . c_{0} \\
\text { L. }+1.7 .50 . d_{0} \\
\text { S. }+0.44 .7 . c_{0}\end{array}\right.$ \\
\hline
\end{tabular}




\section{Triangles de la Méridienne de Quito.}

VII.

HaUteurs

\& Abaiffemens refpectifs

des Signaux.

Toifes.

N. - $\left.525,4^{\circ}\right\}$ Y. $94^{\circ} 17^{\prime} 4^{\prime \prime} 8^{\prime \prime}$

D. - 146,01

$\left.\begin{array}{l}\text { Y. }+525,46 \\ \text { D. }+384,58\end{array}\right\}$ N. $58.23 \cdot 16$

Y. + 146,01

N. $-384,58$

D. 27.18 .56

D. $+157,00\}$ I. ${ }^{\circ} 63 \cdot 39.7$

Z. $-130,40\}$

I. $-157,00\}$ D. 48.31 .45

Z. $-281,97$

$\left.\begin{array}{l}\text { I. }+130,4^{\circ} \\ \text { D. }+281,97\end{array}\right\}$ Z. 67.49 .8

Z. $-281,97\}$ D. $47 \cdot 26.43$

$\frac{\text { L. }+138,21}{\text { D. }+281,97 \text { ? }}$

L. $+423,20\}$ Z. 52. I. 32

$\left.\begin{array}{l}\text { D. }-138,21 \\ \text { Z. }-4^{2} 3,20\end{array}\right\}$ L. 80.31 .45

L. +.423,20,\{Z. 71. 3. 38

S. $+357,95\}$

$\left.\begin{array}{l}\text { Z. }-423,20 \\ \text { S. }-64,06\end{array}\right\}$ L. $47 \cdot 45 \cdot 33$

Z. $-357,95$ S. 61. 10. 49

L. $+64,06\}$

S. $-64,06$ ?

C. $-278,15$

$\left.\begin{array}{l}\text { L. }+64,06 \\ \text { C. }-214,26\end{array}\right\}$

C. $-214,26$

L. $+278,15$

S. $+214,26$ C. $57 \cdot 50.57$
I X.

LONGUEUR des côtés horizontaux, Jes côtés des Triangles réduits au niveau de Carabourou.

Toires.

ND. " 19338,78

Y D. 16515,44

YN. $\quad 8899,40$

D Z. I 6437,8 I

I Z. 13744,6 I

I D. $\quad 16,85,94$

Z L. 12275,95

D L. 13136,75

D Z. 16437,8 I

LS. $\quad 13252,85$

ZS. 10373,04

Z L. 12275,95

8o. 14. 28

o. $\quad 17 . \quad 17$

S. E. \& par 3 obfer. réd.

०. 17.19

47. 44. 0

51. 59. 59

9. 10.50

8o. 14.28

S. O.\& par obferv. réd.

8o. 14. 35

SC. 14352,69

72. 19.42

LC. 12926,82

L S. 13252,85
14. 28.45 S. $O$.

51. 59.59 S. 0 . S. E. S. E. S. 0 . s. 0 .

X I.

Distance entre

les Parallèles des Signaux.

des.

les Méridiens des Signaux. Toifes. 6612,42

1 $817^{2,9} 6$

12139,39

I 1197,99

5526,97

6974,97

11055,79

12164,36

I 2908,67

4720,21

I $8,52,89$

16884,57

2080,80

12098,32

13136,59

$6.6,04$

I 1055,79

12164,36

8150,32

10443,34

10240,11

1654,97

2080,80

12098,32 S. E.
4356,93

I 3675,41

3232,07

$125 \times 6,25$

8159,32

I 0443,34 


\section{Suite de la TABLE du Calcul des}

\begin{tabular}{|c|c|c|c|c|c|}
\hline $\begin{array}{c}\text { I. } \\
\text { ORDRE \& PLANS } \\
\text { des } \\
\text { TRIA NंGLES. }\end{array}$ & $\begin{array}{c}\text { I I. } \\
\text { Noms DES LIEUX } \\
\text { où étoient pofés } \\
\text { les Signaux. }\end{array}$ & $\begin{array}{c}\text { I I I. } \\
\text { A NGLES } \\
\text { DE POSITION } \\
\text { oblervés. }\end{array}$ & $\mid \begin{array}{l}\text { I V. } \\
\text { Equation } \\
\text { pour } \\
\text { ia formen } \\
\text { des } \\
\text {; Angles }\end{array}$ & $\begin{array}{c}\text { V. } \\
\text { LONG U E U R } \\
\text { des } \\
\text { côtés oppofés } \\
\text { aux Angles obfervés. }\end{array}$ & $\begin{array}{c}\text { VI. } \\
\text { ANGLES } \\
\text { de hauteur \& de } \\
\text { dépreffionapparente } \\
\text { obferves. }\end{array}$ \\
\hline & $\begin{array}{l}\text { Senegoalap........ } \\
\text { Choujä̈............. } \\
\text { Sacha-tian-loma.. }\end{array}$ & 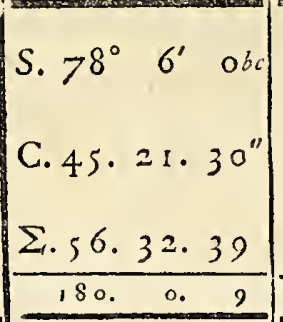 & $\begin{array}{l}-3 \\
-3 \\
-3 \\
-2\end{array}$ & 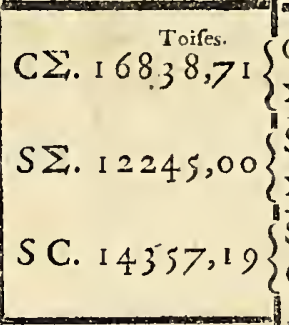 & 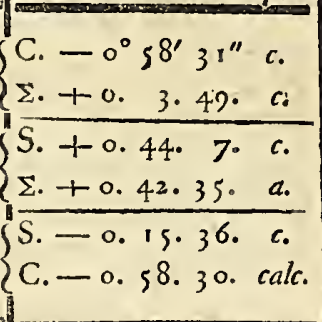 \\
\hline & $\begin{array}{c}\text { Choujäi............. } \\
\text { Sacha-tian-loma.. } \\
\text { ' } \\
\text { Sinaçaouan........ }\end{array}$ & $\begin{array}{l}\text { C. } 50.53 \cdot 79 \\
\Sigma .51 .55 .31 \\
5.77 .11 .31 \\
180.00 .99\end{array}$ & $\begin{array}{l}-3 \\
-3 \\
-3 \\
-9 \\
\end{array}$ & $\begin{array}{l}\Sigma S .13398,15 \\
\text { CS. } 13593,68\} \\
\text { C } \Sigma .16838,71\end{array}$ & 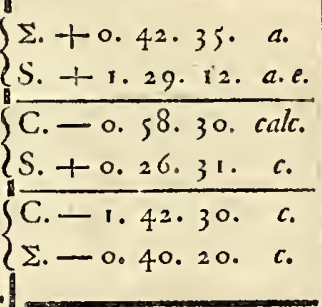 \\
\hline & $\begin{array}{l}\text { Sacha-tian-loma.. } \\
\text { Sinaçaouan ........ } \\
\text { Quinoz-loma..... }\end{array}$ & $\begin{array}{l}\sum .56 .59 .5 j \\
S .50 .38 .46 \\
\frac{Q .72 .21 .22 b}{180.00}\end{array}$ & $\begin{array}{l}-1 \\
-1 \\
-1 \\
-3\end{array}$ & $\begin{array}{l}\text { SQ. } 11791,10 \\
\Sigma Q .10871,42 \\
\Sigma S .13398,15\end{array}$ & 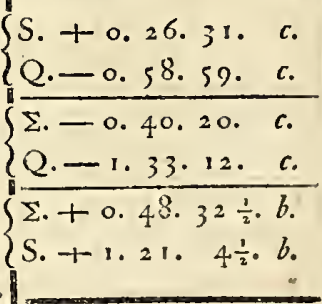 \\
\hline & $\begin{array}{l}\text { Sinaçaouan ........ } \\
\text { Quinoa-loma...... } \\
\text { Boucran ............ }\end{array}$ & 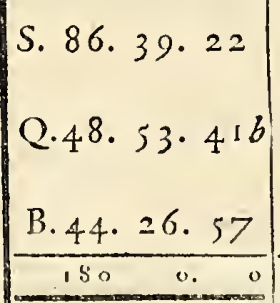 & 0 & $\begin{array}{l}\text { Q.B. } 16809,12 \\
\text { SB. } 12687,32 \\
\text { SQ. } 11791,10\}\end{array}$ & 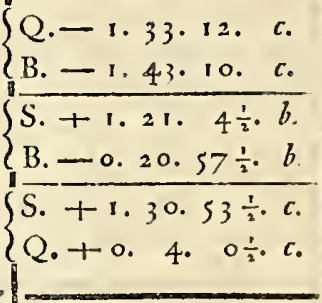 \\
\hline & $\begin{array}{l}\text { Quinoa-loma..... } \\
\text { Boueran............. } \\
\text { Yaflouai............. }\end{array}$ & $\left|\begin{array}{l}\mathrm{Q} .47 \cdot 25 \cdot 1 b \\
\mathrm{~B} .47 \cdot 12.6 \\
\frac{\mathrm{Y} .85 \cdot 22.58}{180.0 .5}\end{array}\right|$ & $\begin{array}{l}-1 \\
-2 \\
-2 \\
-5\end{array}$ & $\begin{array}{l}\text { BY. I } 2416,76 \\
\text { QY. 12373,74 } \\
\text { QB. } 16809,12\end{array}$ & 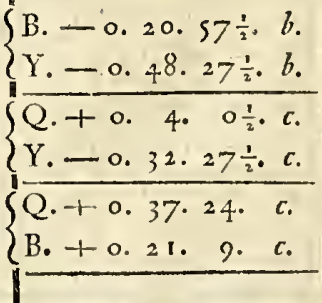 \\
\hline
\end{tabular}




\section{Triangles de la Méridienne de Quito.}

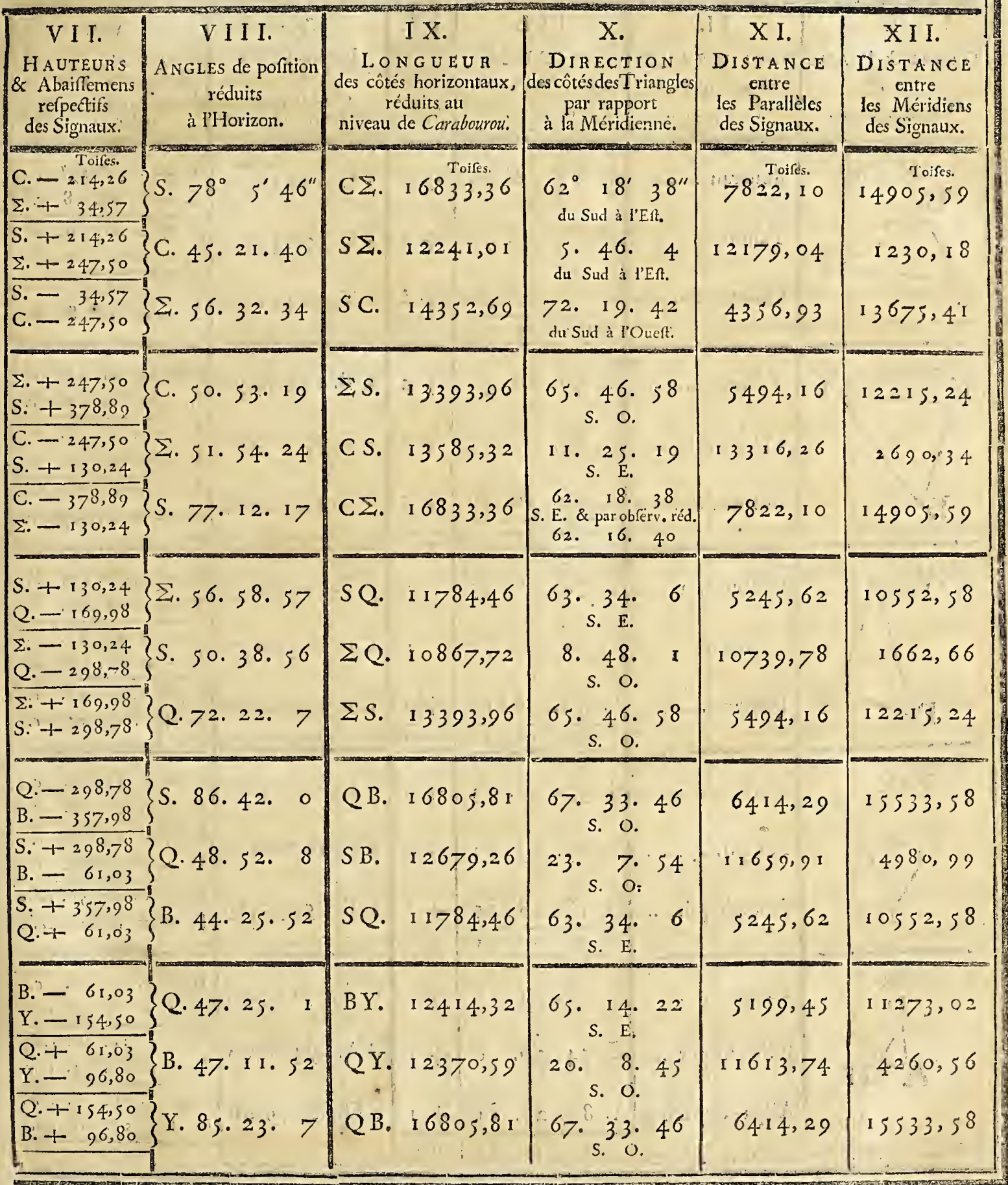




\begin{tabular}{|c|c|c|c|c|c|}
\hline & & Suite de & 7 & $B L E d u$ & Calcul des \\
\hline $\begin{array}{c}\text { ORDRE \& PLANS } \\
\text { des } \\
\text { TRIANGLES. }\end{array}$ & $\begin{array}{c}\text { I I. } \\
\text { NomS DES LIEUX } \\
\text { où étoient pofés } \\
\text { les Signaux. }\end{array}$ & $\begin{array}{c}\text { I I I. } \\
\text { A NGLES } \\
\text { DE POSITION } \\
\text { obfervés. }\end{array}$ & \begin{tabular}{|c|} 
I V. \\
Equation \\
pour \\
la foumint \\
des \\
3 Angles \\
\end{tabular} & $\begin{array}{c}\text { V. } \\
\text { LONG UEUR } \\
\text { des } \\
\text { côtés oppofés } \\
\text { aux Angles oblervés. }\end{array}$ & \begin{tabular}{|c|c|} 
VI. \\
A N GLES \\
de hauteur \& de \\
dépreffion apparente \\
oblervés.
\end{tabular} \\
\hline & $\begin{array}{l}\text { Boueran ............. } \\
\text { Yaffouai........... } \\
\text { Cahouapata......... }\end{array}$ & $\begin{array}{l}\text { B. } 85^{\circ} 6^{\prime} 43^{\prime \prime} \\
\text { Y. } 32.55 \cdot 40 \\
\text { C. } 61.57 \cdot 31 \\
179 \cdot 59 \cdot 54 \\
\end{array}$ & $\begin{array}{l}+2 " \\
+2 \\
+2 \\
+6 \\
\end{array}$ & 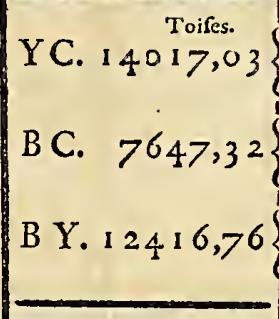 & $\left\{\begin{array}{l}Y .-0^{\circ} 32^{\prime} 27^{\prime \prime} \frac{1}{2} \cdot c . \\
C_{0}-1.14 .38 c . \\
\text { B. }+0.21 .9 . c \\
\text { C. }-0.21 .13 . c . \\
\text { B. }+1.6 .55 . d . \\
Y .+0.90^{\circ} 8 . d .\end{array}\right.$ \\
\hline & $\begin{array}{l}\text { Yaffouai............ } \\
\text { Cahouapata....... } \\
\text { Borma.............. } \\
\end{array}$ & $\begin{array}{l}Y .49 \cdot 20 \cdot 47 \\
\text { C. } 77 \cdot 42 \cdot 17 \\
\text { B. } 52 \cdot 56 \cdot 49 \\
179 \cdot 59 \cdot 53 \\
\end{array}$ & $\begin{array}{l}+2 \\
+2 \\
+3 \\
+7 \\
\end{array}$ & $\begin{array}{l}\text { C B. } 13324,72 \\
\text { Y B. } 17160,48 \\
\text { YC. } 14017,03\end{array}$ & 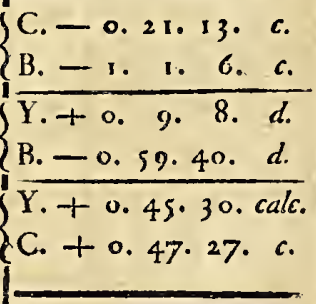 \\
\hline & $\begin{array}{l}\text { Cahouapata..... } \\
\text { Borma............ } \\
\text { Pougin........... }\end{array}$ & $\begin{array}{l}\text { C. } 34.8 .45 \\
\text { B. } 91.44 .57 \\
\text { P. } 54.6 .17 \\
179.5959 \\
\end{array}$ & $\begin{array}{r}0 \\
+1 \\
+1 \\
\end{array}$ & $\begin{array}{l}\text { BP. } 9232,49 \\
\text { CP. } 16440,71 \\
\text { CB. } 13324,72\end{array}$ & 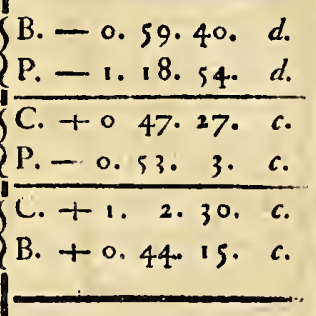 \\
\hline & $\begin{array}{l}\text { Borma.............. } \\
\text { Pougin............. } \\
\text { Pillatchiquir..... }\end{array}$ & $\left\{\begin{array}{l}\text { B. } 37 \cdot 47 \cdot 33 \\
\text { ․ } 83 \cdot 53 \cdot 43 \\
\frac{58 \cdot 18}{17959} \frac{39}{55}\end{array}\right.$ & $\begin{array}{l}+1 \\
+2 \\
+2 \\
+5 \\
\end{array}$ & $\begin{array}{l}\text { РП. } 6649,00 \\
\text { ВП. } 10788,55 \\
\text { В Р. } 9232,49\end{array}$ & $\left\{\begin{array}{l}\text { P. }\left\{\begin{array}{l}\text { I. }+0.31 .47 . c \\
\text { B. }+0.44 .15 . c \\
\text { I. }+2.3 .47 . c\end{array}\right. \\
\begin{array}{l}\text { B. }-0.41 .50 . \text { calc. } \\
\text { P. } 2.10 .20 . \text { cals. }\end{array}\end{array}\right.$ \\
\hline & $\begin{array}{l}\text { Pougin ............. } \\
\text { Pillatchiquir ...... } \\
\text { Ailpa-roupachca. }\end{array}$ & $\begin{array}{l}\text { P. } 38.4 \cdot 36 \\
\text { П. } 54 \cdot 30 \cdot 6 \\
\frac{\text { A. } 87 \cdot 25 \cdot 14}{17954 \cdot 56}\end{array}$ & $\begin{array}{l}+2 \\
+1 \\
+1 \\
++t\end{array}$ & $\begin{array}{l}\text { П А. } 4104,75 \\
\text { РА. } 5418,68 \\
\text { РП. } 6649,00\end{array}$ & 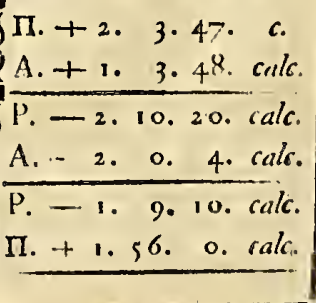 \\
\hline
\end{tabular}




\section{Triangles de la Méridienne de Quito.}

\begin{tabular}{|c|c|c|c|c|c|c|}
\hline $\begin{array}{c}\text { V I I. } \\
\text { H A UT Eurs } \\
\text { \& A baiffemens } \\
\text { refpectifs } \\
\text { des Signaux. }\end{array}$ & $\begin{array}{c}\text { V I I I. } \\
\text { ANGLES de poffition } \\
\text { réduits } \\
\text { à l'Horizon. }\end{array}$ & $\begin{array}{r}\text { Lo } \\
\text { des côt } \\
r \\
\text { niveau }\end{array}$ & $\begin{array}{l}\text { I X. } \\
\text { NG U E U R } \\
\text { és horizontaux, } \\
\text { éduits au } \\
\text { de Carabourou. }\end{array}$ & \begin{tabular}{|c|} 
X. \\
DiRECTION \\
descôtés des I riangles \\
par rapport \\
à la Méridienne.
\end{tabular} & $\begin{array}{c}\text { XI. } \\
\text { Dis TA NCE } \\
\text { entre } \\
\text { les Parallèles } \\
\text { des Signaux. }\end{array}$ & $\begin{array}{c}\text { X I I. } \\
\text { Dis TA nCE } \\
\text { entre } \\
\text { les Méridiens } \\
\text { des Signaux. }\end{array}$ \\
\hline $\begin{array}{l}\text { Toiles. } \\
Y .-\quad 96,80 \\
\text { C. }-157,41\end{array}$ & B. $85^{\circ} 7^{\prime} 21^{\prime \prime}$ & YC. & $\begin{array}{c}\text { Toires. } \\
14014,84\end{array}$ & $\begin{array}{lll}81^{\circ} & 50^{\prime} & 22^{\prime \prime} \\
\text { du jud à l'Ouet. }\end{array}$ & $\begin{array}{c}\text { Toifes. } \\
1989,37\end{array}$ & $\begin{array}{c}\text { Toifes. } \\
1387^{2}, 93\end{array}$ \\
\hline $\begin{array}{l}\text { B. }+96,80 \\
\text { C. }-6_{1}, 86 \\
\end{array}$ & $Y .32 .55 .16$ & B C. & 7644.52 & $\left|\begin{array}{ccc}19 . & 52 . & 59 \\
\text { S. O. \& par obferi. réd. } \\
19 . & 54 . & 17\end{array}\right|$ & $7 \times 88,82$ & 2599.21 \\
\hline $\begin{array}{l}\text { B. }+157,41 \\
\text { Y. }+61,86\end{array}$ & C. $61.57 \cdot 23$ & B Y. & 12414,32 & $\begin{array}{ccc}65 . & 14 & 22 \\
\text { S. E. \& par } & \text { ublerv. red } \\
65 . & 1.3 . & 44 \\
\end{array}$ & 5199,45 & 11273,02 \\
\hline $\begin{array}{l}\text { C. }-61,86 \\
\text { B. }-266,00\end{array}$ & 49.20 .46 & $C B$. & 13321,54 & $\begin{array}{l}\text { 20. } 27.35 \\
\text { S. E. }\end{array}$ & 12481,20 & 4656,53 \\
\hline $\begin{array}{r}\text { Y. }+61,86 \\
\text { B. }-207,54 \\
\end{array}$ & C. 77.42 .3 & Y B. & $171,6,32$ & $\begin{array}{l}\text { 32. } 29 \cdot 36 \\
\text { s. } 0 .\end{array}$ & 14470,56 & 9216,41 \\
\hline $\left.\begin{array}{l}Y .+266,00 \\
\text { C. }+207,5+\end{array}\right\}$ & 52.57 .11 & Y C. & 14014,84 & $\begin{array}{l}81 . \text { so. } 22 \\
\text { s. } 0 .\end{array}$ & 1989,37 & 13872,93 \\
\hline $\begin{array}{l}\text { B. }-207,54 \\
\text { P. }-338,03\end{array}$ & 34.9 .5 & B P. & 9230,90 & $\begin{array}{l}67.48 \cdot 10 \\
\text { s. } 0 .\end{array}$ & 3487,40 & $8 ; 46,79$ \\
\hline $\begin{array}{l}\text { C. }+207,54 \\
\text { P. }-130,62\end{array}$ & B. 91.44 .15 & C P. & 16435,63 & $\begin{array}{c}\text { 13. } 41.30 \\
\text { s. } 0 .\end{array}$ & 15968,60 & $38,0,26$ \\
\hline $\begin{array}{l}\text { C. }+338,03 \\
\text { B. }+130,62\end{array}$ & 54.6 & C B. & 13321,54 & $\begin{array}{l}20.27 \cdot 35 \\
\text { S. E. }\end{array}$ & 12481,20 & 4656,53 \\
\hline $\begin{array}{l}\text { P. }-130,62 \\
\Pi 1+115,49 \\
\end{array}$ & B. $37 \cdot 46.3$ & РП. & 6643,85 & $\begin{array}{l}\text { 28. 16. } 45 \\
\text { S. E. }\end{array}$ & 5850,91 & 3147,65 \\
\hline $\begin{array}{l}\text { B. }+130,62 \\
\text { II. }+245,64 \\
\end{array}$ & P. 83.55 .5 & ВП. & $10786,7^{8}$ & $\begin{array}{lll}\text { 30. } & 2.7 \\
\text { s. } & 0 .\end{array}$ & $933^{8,31}$ & 5399,14 \\
\hline $\begin{array}{l}\text { B. }-115,49 \\
\text { P. }-245,64\end{array}$ & M. 58.18 .52 & B P. & 9230,90 & $\begin{array}{l}67.48 .10 \\
\text { s. } 0 .\end{array}$ & $3487,4^{\circ}$ & 8546,79 \\
\hline $\begin{array}{l}\text { H. }+245,64 \\
\text { A. }+104,76\end{array}$ & P. $38.4 \cdot 4^{2}$ & $\Pi A$. & 4101,81 & $\begin{array}{l}\text { 82. } 49 \cdot 18 \\
\text { N. } 0 .\end{array}$ & 512,55 & 4069,66 \\
\hline $\begin{array}{l}\text { P. }-245,64 \\
\text { A. }-140,88 \\
\end{array}$ & П1. 54.32 .33 & PA. & 5417,39 & $\begin{array}{l}\text { 9. } 47 \cdot 57 \\
\text { s. } 0 .\end{array}$ & 5338,35 & 922,01 \\
\hline $\begin{array}{r}P-104,76 \\
\text { II. }+140,88 \\
\end{array}$ & A. 87.22 .45 & PП. & 6643,85 & $\begin{array}{l}28.16 .45 \\
\text { s. E. }\end{array}$ & 5850,91 & $3.147,65$ \\
\hline
\end{tabular}




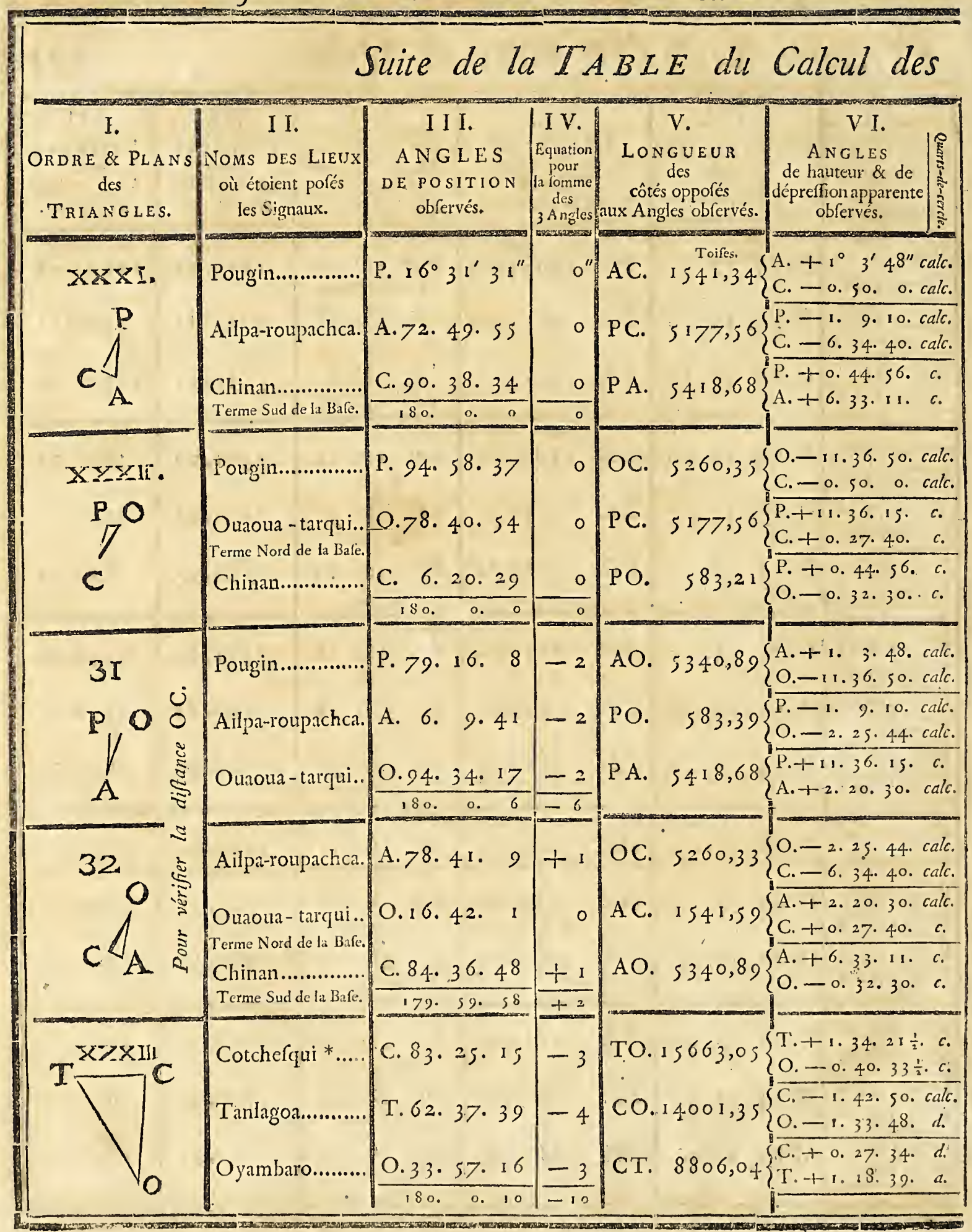

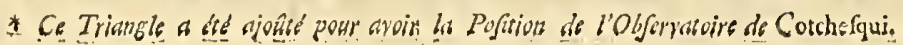




\section{Triangles de la Méridienne de Quito.}

\begin{tabular}{|c|c|c|c|c|c|c|}
\hline $\begin{array}{l}\text { VI I. } \\
\text { H A UTEURs } \\
\text { \& Abaiffemens } \\
\text { refpectifs } \\
\text { des Signaux. }\end{array}$ & $\begin{array}{c}\text { V I I I. } \\
\text { ANGLES de pofition } \\
\text { réduits } \\
\text { à l'Horizon. }\end{array}$ & $\begin{array}{r}\text { Lo } \\
\text { des côt } \\
\vdots \quad r \\
\text { niveau }\end{array}$ & $\begin{array}{l}\text { I X. } \\
\text { \& U EU R } \\
\text { horizontaux, } \\
\text { duits au } \\
\text { de Carabourou. }\end{array}$ & $\begin{array}{c}\text { X. } \\
\text { DIR ECTION } \\
\text { des côtés desTriangles } \\
\text { par rapport } \\
\text { à la Méridienne. }\end{array}$ & $\begin{array}{c}\text { X I. } \\
\text { Dis T A N E } \\
\text { entre } \\
\text { Jes Parallèles } \\
\text { des Signaux. }\end{array}$ & $\begin{array}{c}\text { X I I. } \\
\text { DIS T A N C E } \\
\text { entre } \\
\text { les Méridiens } \\
\text { des Signaux. }\end{array}$ \\
\hline $\begin{array}{l}\text { Toifes. } \\
\text { A. }+104,76 \\
\text { C. }-71,47 \\
\end{array}$ & P. $16^{\circ} 25^{\circ} \quad I^{\prime \prime}$ & A C. & $\begin{array}{c}\text { Toifes. } \\
1531,22\end{array}$ & $\begin{array}{l}63^{\circ} 3^{\prime} 3^{\prime \prime} \\
\text { du Nord a l'Oueft. }\end{array}$ & $\begin{array}{c}\text { Toires. } \\
693,95\end{array}$ & $\begin{array}{c}\text { Toires. } \\
\text { I }_{3} 64,92\end{array}$ \\
\hline $\begin{array}{l}\text { P. }-104,76 \\
\text { C. }-1>6,23 \\
\end{array}$ & A. 72.51 .0 & PC. & 5176,94 & $\begin{array}{l}\text { 26. I 2. } 58 \\
\text { du Sud à l'Outen. }\end{array}$ & $4644,4 x$ & 2286,95 \\
\hline $\begin{array}{l}\text { P. }+71,47 \\
\text { A. }+17^{6,23}\end{array}$ & $\{$ C. $90.43 \cdot 59$ & PA. & 5417,39 & $\begin{array}{l}\text { 9. } 47.57 \\
\text { du Sud à l'Oueft. }\end{array}$ & 5338,35 & 922,01 \\
\hline $\begin{array}{l}\text { O. }-117,37 \\
\text { C. }-7 \times, 47 \\
\end{array}$ & P. 95. I S.II & OC. & 5260,09 & $\begin{array}{c}32.25 \cdot 29 \\
\text { s. } 0 .\end{array}$ & 4440,03 & $2820,4^{2}$ \\
\hline $\begin{array}{l}\text { P. }+117.37 \\
\text { C. }+46.02 \\
\end{array}$ & $\left\{0.78 .3^{2.18}\right.$ & P C. & 5176,94 & $\begin{array}{l}\text { 26. I } 2.58 \\
\text { s. } 0 .\end{array}$ & 4644,41 & 2286,95 \\
\hline $\begin{array}{l}\text { P. }+71,47 \\
0 .-46,02\end{array}$ & C. 6.12 .31 & PO. & 571,27 & $\begin{array}{ll}\text { 69. 2. I } 3 \\
\text { s. E. }\end{array}$ & 204,38 & 533,45 \\
\hline $\begin{array}{l}\text { A. }+104.76 \\
\text { O. }-117.37 \\
\end{array}$ & $78.5 \mathrm{I} \cdot \mathrm{I}$ & AO. & 5336,70 & $\begin{array}{c}\text { I 5. } 47 \cdot 5 \text { I } \\
\text { N. E. }\end{array}$ & 5135,13 & 1452,85 \\
\hline $\begin{array}{l}\text { P. }-104,76 \\
0 .-222,25 \\
\end{array}$ & A. 5.59 .54 & PO. & 568,41 & 69. 3. 4 & 203,22 & 530,83 \\
\hline $\begin{array}{l}\text { P. }+117,37 \\
\text { A. }+322,25\end{array}$ & 0.95 .9 .5 & PA. & 5417,39 & $\begin{array}{l}\text { S. } E_{*} \\
\text { 9. } 47 \cdot 57 \\
\text { s. } 0 .\end{array}$ & $533^{8,35}$ & 922,01 \\
\hline $\begin{array}{l}\text { O. }-222,25 \\
\text { C. }-17^{6,23}\end{array}$ & A. $7^{8} .50 .54$ & OC. & 5259,94 & S. $3^{2 .}$ \& 23 par ubferv. réd. 53 & 4439,90 & 2820,34 \\
\hline $\begin{array}{l}\text { A. }+222,25 \\
\text { C. }+46,02 \\
\end{array}$ & O. $16.37 \cdot 38$ & AC. & 1534,07 & $\begin{array}{ccc}32 . & 23 . & 47 \\
63 \cdot & 5 \cdot 14\end{array}$ & 695,24 & I $367,4^{8}$ \\
\hline $\begin{array}{l}\text { A. }+176,23 \\
\text { O. }-46,02\end{array}$ & C. $84 \cdot 31.28$ & AO. & 5336,70 & $\begin{array}{l}\text { I S. } 47.5 \text { I } \\
\text { N. E. }\end{array}$ & 5135,13 & 1452,85 \\
\hline $\begin{array}{l}\text { T. }+252,54 \\
\text { O. }-138,75 \\
\end{array}$ & $83 \cdot 23 \cdot 53$ & TO. & Is 657,28 & $\begin{array}{ll}30 . & 2.22 \\
\text { S. E. } & \end{array}$ & 13554,21 & $7^{8} 37,98$ \\
\hline $\begin{array}{l}\text { C. }-2,52,54 \\
\text { O. }-392,82\end{array}$ & $\{$ T. 62.39.16 & $\mathrm{CO}$. & 14000,46 & 3. $54 \cdot 29$ & I 3967,90 & 954,21 \\
\hline $\begin{array}{l}\text { C. }+13^{8,75} \\
\text { T. }+392,82 \\
\end{array}$ & $0.33 .56 .5 \mathrm{I}$ & CT. & $880 \mathrm{I}, 9 \mathrm{I}$ & $\begin{array}{l}\text { s. } 0 . \\
\text { s. } 8.22 \\
\text { s. } 0 .\end{array}$ & $4: 3,69$ & 8792,19 \\
\hline
\end{tabular}




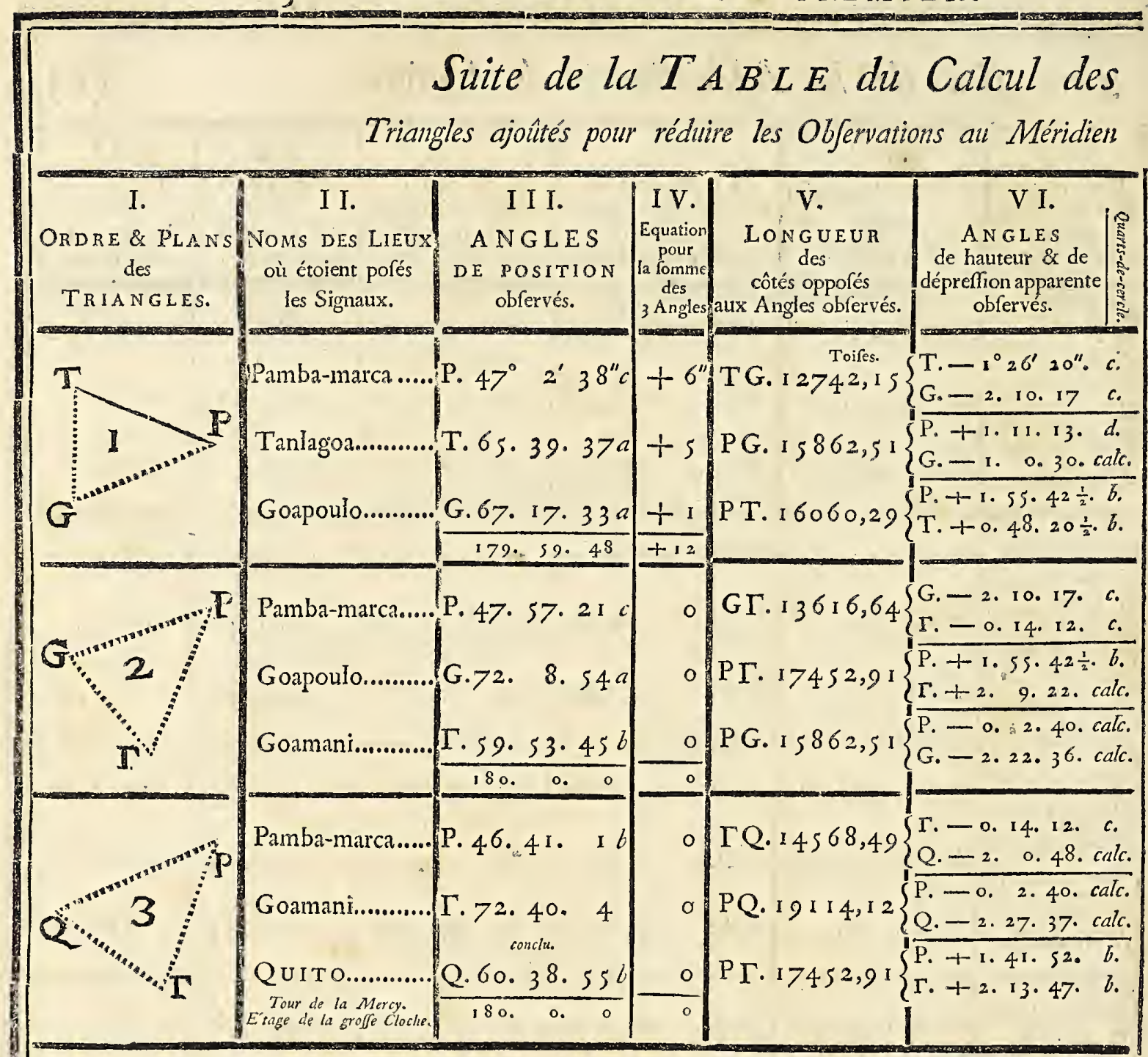

Ces trois derniers Triangles ont été tirés de la 


\section{Triangles de la Méridienne de Quito.} o au Paraliele de la Tour de la Mercy de Quito.

\begin{tabular}{|c|c|c|c|c|c|}
\hline $\begin{array}{c}\text { VI I. } \\
\text { H A UT EURs } \\
\text { \& A baiffemens } \\
\text { refpedtifs } \\
\text { des Signaux. }\end{array}$ & $\begin{array}{c}\text { V I I I. } \\
\text { ANGLES de pofition } \\
\text { réduits } \\
\text { à l'Horizon. }\end{array}$ & $\begin{array}{c}\text { I X. } \\
\text { LONGUUEUR } \\
\text { des côtés horizontaux, } \\
\text { réduits au } \\
\text { niveau de Carabourou. }\end{array}$ & $\begin{array}{c}\text { X. } \\
\text { D I R ECT ION } \\
\text { descôtés desTriangles } \\
\text { par rapport } \\
\text { à la Méridienne. }\end{array}$ & $\begin{array}{c}\text { XI. } \\
\text { DIS TA NCE } \\
\text { entre } \\
\text { les Parallèles } \\
\text { des Signaux. }\end{array}$ & $\begin{array}{l}\text { X I I. } \\
\text { D I T TA N CE } \\
\text { entre } \\
\text { les Méridiens } \\
\text { des Signaux. }\end{array}$ \\
\hline $\begin{array}{r}\text { Toifes. } \\
\text { T. }-367,98 \\
\text { G. }-5^{6} 7,7^{8}\end{array}$ & P. $47^{\circ}-3^{\prime} 54^{\prime \prime}$ & T G. 12739,35 & $\begin{array}{l}0^{\circ} 20^{\prime} 57^{\prime \prime} \\
\text { du Sud à l'E } E f_{0} .\end{array}$ & $\begin{array}{c}\text { Toires. } \\
12739, \text { I } 2\end{array}$ & $\begin{array}{c}\text { Toires. } \\
77,63\end{array}$ \\
\hline $\begin{array}{l}\text { P. }+367,98 \\
\text { G. }-201,70 \\
\end{array}$ & T. 65.37 .45 & PG. $\quad$ I 5849,98 & $\begin{array}{l}\text { 66. } 57.24 \\
\text { du Sud à t'Oueft. }\end{array}$ & 6204,12 & 14585,29 \\
\hline $\begin{array}{l}\text { P. }+567,78 \\
\text { T. }+201,70\end{array}$ & G. 67.18 .21 & PT. 16053,26 & $\begin{array}{l}65.58 .42 \\
\text { du Nord a l'Ouent. }\end{array}$ & 6535,00 & 14662,91 \\
\hline $\begin{array}{l}\text { G. }-56,7,78 \\
\text { r. - 29,29 } \\
\end{array}$ & $47 \cdot 55 \cdot 49$ & GI. I3604,12 & $\begin{array}{l}\text { 40. 50. } 33 \\
\text { S. E. }\end{array}$ & 10291,65 & $88,6,85$ \\
\hline $\begin{array}{l}\text { P. }+567,78 \\
\Gamma .+538,49 \\
\end{array}$ & G.72. 1 2. 3 & РГ. $\quad 17449,03$ & $\begin{array}{ll}\text { I } 9 . & 1.35 \\
\text { S. } & \text { O. }\end{array}$ & 16495,77 & 5688,44 \\
\hline $\begin{array}{l}\text { P. }+29,29 \\
\text { G. }-538,49\end{array}$ & Г. 59.52. & PG. $15849 ; 98$ & $\begin{array}{l}\text { 66. } 57 \cdot 24 \\
\mathrm{s.} 0 .\end{array}$ & 6204,12 & 14585,29 \\
\hline $\begin{array}{l}\text { T. } 29,29 \\
\text { Q. } 618,91 \\
\end{array}$ & P. $46.39 .4 \mathrm{I}$ & $\Gamma \mathrm{C} . \mathrm{I}_{4554,64}$ & $\begin{array}{c}53 \cdot 37 \cdot 36 \\
\text { N. } \mathrm{O} .\end{array}$ & 8631,55 & 11718,97 \\
\hline $\begin{array}{l}\text { P. }+29,29 \\
\text { Q. }-596,09 \\
\end{array}$ & 72.39 .11 & PQ. $\quad 19101,42$ & $\begin{array}{c}65 \cdot 41 \cdot{ }^{6} \\
\text { s. } 0 .\end{array}$ & $.7^{86} 4,22$ & 17407,42 \\
\hline $\begin{array}{l}\text { P. +618,91 } \\
\text { Г. }+596,09\end{array}$ & $0.60 .4 \mathrm{I}$. & PГ. $\quad 17449,03$ & $\begin{array}{lll}\text { 19. } & \text { 1. } 35 \\
\text { s. } & 0 .\end{array}$ & 16495,77 & 5688,44 \\
\hline
\end{tabular}

fuice mefurée particulièrement par M. Godin. 


\section{A R T I C L E VII.}

Explication de la Colonne I de la Table: Ordre \& plan des Triangles.

LA fuite \& l'enchaînement des Triangles de la Méridienne fe voit dans la planche 1I, qui les repréfente tous; mais j’ai cru outre cela qu'en cherchant dans la Table la mefure d'un angle, Ia longueur ou la direction d'un côté, \&c. il feroit commode pour le Lecteur d'avoir fous les yeux la figure du Triangle qu'il confidère, \& d'en trouver le plan vis-à-vis des calculs qui lui répondent.

Ce font ces plans ifolés des Triangles, orientés \& placés felon l'ordre des obfervations, qui compolent la première Co. lonne de la Table.

Dans le calcul de chaque Triangle, j’ai défigné les Signaux correfpondans par la lettre initiale de leur nom; \& dans le petit nombre de cas où les noms de deux Signaux d'un même Triangle ont commencé par la même lettre, j’ai employé pour Pun des deux une lettre grecque.

\section{A R T I C L E VIII.}

'Explication de la Colonne II: Noms des lieux où étoient placés les Signaux.

EN France, la multitude des objets, tels que les clochers; châteaux, moulins, arbres ifolés, donnoit fouvent à l'Obfervateur la liberté de choifu le point qui lui convenoit le mieux 
pour former fes Triangles; mais dans le pays où nous avons opéré, les montagnes fur lefquelles nos Triangles devoient s'appuyer néceflairement, ne nous offroient pas de point fixe, \& il nous a fallu poler des Signaux artificiels : nous les formions d'abord fuivant la nature du terrein, tantôt avec-des pièces de bois dreffées en pyramides \& couvertes de paille, tantôt en élevant des maffes de pierres cylindriques ou coniques; ceux de bois étoient fujets à être fouvent enlevés dans les lieux où le bois étoit rare; \& tel Signal, comme celui de Pamba-marca, a été, par différens accidens, renouvelé jufquà fept fois.

Pendant mon derrier féjour fur cette montagne, je m'avifai de faire raffembler par les Indiens qui nous fervoient, un grand nombre de pierres des ruines d'une ancienne fortifrcation des naturels du pays, \& d'en former une efpèce de tourelle que je rendis refpectable en la faifant fervir de bafe à une croix de 18 pieds de haut. Ce Signal nous a fervi depuis en plufieurs occafions, \& nous a difpenfés de retourner fur la montagne: il fubfiftoit encore cinq ans après, lorfque je partis de Quito.

Après nos premiers Triangles, nous convînmes, conformément à la propofition qu'en fit M. Godin, de nous fervir déformais pour Signaux, de tentes ou de canonières qui avoient cet avantage, que lors même que vûes d'un lieu plus élevé, elles fe projetoient fur le terrein, leur couleur blanche les rendoit apparentes, \& Cervoit à les diftinguer de fort loin.

La feconde Colonne de la Table des Triangles eft une fimple lifte des noms des lieux où étoient pofés les Signaux. Ces noms font Indiens pour I w plufpart; je les ai écrits felon lortho- 
graphe françoife, non feulement parce que j'écris en François, mais encore parce qu'il y a quelques fons communs à la langue Péruvienne \& à la Françoife, qui font totalement inconnus dans la langue Efpagnole, \& que l'orthographe Efpagnole ne peut rendre: tels font ceux que nous exprimons par ch \& par $j$. Un de nos Signaux, par exemple, étoit placé fur une montagne que les Indiens nomment Choujaï. Il eft impof fible d'écrire ce mot en Efpagnol, fans en changer totalement da prononciation.

Dans les noms Efpagnols, comme Cuenca, qui fe prononce Coinca, \&x. j'ai fuivi l'orthographe Efpagnole pour ne les pas rendre méconnoiffables aux yeux, \& ils font écrits en carac: tère italique.

Je crois devoir avertir le Lecteur, pour prévenir toute équivoque, que les endroits où nous avons pofé des Signaux nous ont quelquerois été défignés fous différens noms. Un Pâtre Indien fe croit en droit d'impofer des noms à fa fantaifie à des lieux que lui feul fréquente, \& tous font dans l'ufage d'en donner à chaque pièce de terre \& à chaque colline des montagnes de leur canton. Le Signal qui termine notre Triangle XIV étoit pofé fur le fommet d'une montagne nommée Ygualata; on nous dit que cet endroit en particulier fe nommoit Guayama. Feu M. Maldonado, Seigneur du lieu, m'a plufieurs fois affuré quill s'étoit foigneufement informé de fon Fermier \& de fes Indiens, du nom de Guayama, dont il n'avoit jamais rien pâ découvrir; ce qui m’a déterminé à rendre à ce Signal fon vrai nom d'Ygualata. Peut-être en aurai-je encore nommé quelqu'un autrement que $M$. Bouguer; il y en a qui ont changé deux \& trois fois de nom. 


\section{A R T I C L E IX.}

Explication de la Colonne III: Angles de pofition obfervés.

Dans la Table de mes angles obfervés \& corrigés, dont je donnai copie au mois d'Avril I 74 I à Mrs Godin \& Bouguer, Jes différentes équations qui réfultoient des diverfes corrections précédemment expliquées, faifoient autant de Colonnes féparées; j’ai craint quiil ne parût plus d'affectation que d'utilité à étaler ici tout ce détail. Je n'ai donc fait, dans la Table cijointe, qu'une feule Colonne des angles qui avoient déjà fubi la correction de l'erreur des divifions, celle du défaut de parallélifme de la Lumette, \& celle de la rẹ́duction au centre. Ainfi on y trouvera les angles tels qu’ils euffent été obfervés, fi le Quartde-cercle eût-été bien divifé, la Lunette bien placée fur le limbe, \& que l'interfection des Lunettes fe fût faite au centre du Signal: il y en a même quelques-uns qui, outre ces corrections communes à tous, ont encore été foûmis à une correction particulière, de la nature de celles que j'ai indiquées à la fin de l'article V. C'eft toûjour's par obfervation, que les erreurs qui font l'objet de la correction, ont été reconnues; j’ai donc pû intituler cette Colonne, Angles de pofition obfervés.

Les lettres $a, b, c$, qui fę trouvent à la fuite de quelques angles, fervent à défigner les différens Quarts-de-cercle avec lefquels ont été obfervés les angles, comme je l'ai déjà remarquéo Tous les autres angles, qui ne font fuivis d'aucune lettre, ont été obfervés avec mon Quart-de-sercle de trois pieds de

$\mathrm{H}$ ij 


\section{MESURE DES TROIS PREMIERS}

rayon (Voyez art. $I V$.) Ce même Quart - de - cercle a été défrgné ailleurs par la lettre $d$; \& un autie d'un pied de rayon, lequel m’appartenoit, \& qui n’a été employé que pour quelques angles de hauteur, a été indiqué par la lettre $e$.

\section{A R T I C L E X.}

Explication de la Colonne IV: Équation pour la fomme des trois angles.

IL arrive fouvent que la fomme des trois angles d'un Triangie, même après toutes les corrections précédentes, n'eft pas encore égale à deux angles droits, comme elle devroit l'être: La quantité dont elle en diffère, partagée également entre les trois angles, eft l'équation indiquée dans la quatrième Colonne de la Table; \& celle-ci eft le réfultat de la quatrième correction, qui a été expliquée dans l'article $V$.

On peut voir par la Table des Triangles, que cette équation, ainf diftribuée, ne monte que très-rarement à quatre fecondes pour chaque angle; qu'ordinairement même elle n'eft que d'une, deux , ou trois fecondes, țantôt en plụs, tantôt en moins, comme il doit arriver aux erreurs dont la caufe n'eft pas conftante; \& enfin que l'équation eft quelquefois nulle. Mais fi I'on fait attention que fur un Quart-de-cercle de trois pieds de rayon, quatre fecondes ne répondent pas à un centième de ligne, \& qu'un cheveu de groffeur oldinaire couvre au moins huit à dix fecondes, on jugera, fans doute, quili n'étoit guère permis d'efpérer une plus grande précifion. 


\section{A R T I C L E XI.}

Explication de la Colonne V: Longueur des côtés oppofés aux angles obfervés.

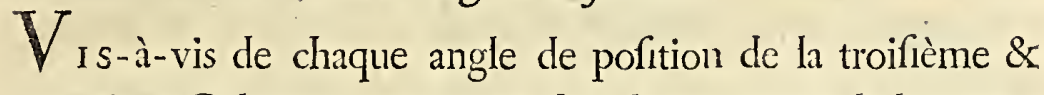
quatrième Colonnes, on trouve dans la cinquième la longueur calculée, en toifes \& en centièmes de toifes, du côté oppofé à cet angle. Cette longueur eft la diftance, en droite ligne, des Signaux entre lefquels l'angle a été obfervé. Par exemple, dans Ia ligne 3 de la troifième Colonne ( $T r$. I) vis-à-vis de l'angle $O$ de $63^{\mathrm{d}} 4^{\prime} 8^{\prime}$ I $6^{\prime \prime}$ obfervé à Oyambaro entre les Signaux $P \& C$ de Pamba-marca \& de Carabourou, l'on trouve dans la cinquième Colonne le nombre 9022,96 , qui exprime en toifes ta longueur du côté $P C$ oppofé à l'angle $O$, c'eft-à-dire, Ja diftance en ligne droite entre les deux Signaux de Pamba-marca \& de Carabourou. Comme un même côté peut être commun à plufieurs Tringles, \& que dans une fuite de Triangles il eft au moins commun aux deux qui fe touchent, le nombre qui exprime la diftance de deux Signaux, ou la longueur d'un côté, ne peut manquer de fe trouver répété dans Ia Table, au moins vis-à-vis des deux angles auxquels le côté eft oppofé, dans deux Triangles différens. On a cru que cette répétition, qui fert de confirmation, déplairoit moins que de Jaiffer des vuides à remplir dans la Table.

Le premier nombre de $\mathrm{la}$ Colonne $\mathrm{V}$ que nous examinons ici ou le premier côté de Triangle 6274,os toifes, n’a pas été obfervé avec le Quart-de-cercle; c'eft la diftance en droite ligne des deux termes de la Bafe, telle qu'elle a été conclue de Ia mefure actuelle. 


\section{A R T I C L E XII.}

Explication de la Colonne VI: Angles verticaux, ou de hauteur \& de déprefion apparente, réciproquement obfervés d'un Signal à l'autre.

LA réduction des angles à un plan horizontal, de laquelle il fera parlé bien-tôt, étoit un point très-capital dans notre travail, vû la grande inégalité du terrein. Cette réduction fuppofe la connoiffance des angles de hauteur ou de dépreffion des objets entre lefquels l'angle de pofition a été obfervé: auffr avons-nous eu grande attention d'obferver la hauteur ou l'abaiflement apparent des Signaux, fans négliger jamais de rectifier le Quart-de-cercle par le renverfement, malgré la difficulté qu'il y avoit à y réuffir, fur des fommets de montagnes, où un vent violent $\&$ continuel ne permettoit pas au filà-plomb de s'arrêter, \& dans des lieux, dont la neige, le verglas \& le froid rendoient fouvent l'accès difficile, \& le féjour infupportable.

Il eft vrai que ces difficultés ont fait, qu'au lieu d'obferver chacun de nous à part les angles verticaux avec nos différens Quarts-de-cercle, comme nous avons fait à l'égard des angles de pofition, nous nous fornmes le plus fouvent contentés, pour abréger, d'obferver les premiers en commun, avec un feul inftrument, en nous aidant mutuellement. Comme nous étions ordinairement enfemble, M. Bouguer \& moi, nous nous fommes fervis le plus fouvent de fon Quart-de-cercle, plus maniable que le mien. Il faut avouer auff que ces angles de hautem n'ont pas toûjours été difcutés avec le même fcrupule que les 
angles de pofition; \& cela n'étoit ni néceffaire, ni quelquefois poffible, fur-tout quand le brouillard, qui commençoit à monter \& à obfcurcir les Signaux, nous obligeoit d'opérer à la hâte. Cependant, comme nous étions alors deux \& trois Obfervateurs, l'un occupé à pointer la Lunette, les autres à caler l'inftrument \& à eftimer le point de la divifion où répondoit le fil-à-plomb; je crois quill eft arrivé rarement qu'il y ait eu une minute d'incertitude : \& par conféquent en prenant le milieu, il ne peut y avoir eu qu'une demi-minute d'erreur à craindre fur la hauteur d'un objet. Je pourrois en excepter un petit nombre de cas, où me trouvant feul, \& ne voulant pas perdre un temps précieux, je ne pouvois employer à la mefure des angles verticaux qu'un petit inftrument d'un pied de rayon : je profitois, pour les obferver, de quelques intervalles Voy, art. $I X$. de temps très-courts, où je ne pouvois faire aucun ufage de mon grand Quart-de-cercle, ni pour mefurer les angles des Triangles, parce que des nuages paffagers me déroboient Ia vîe de quelque Signal; ni pour obferver les angles verticaux, parce que la mefure des angles de pofition exigeoit que le grand Quart-de-cercle fût monté horizontalement. Au refte j’ai évité d'employer aucun de ces angles dans ma Table, quand il n’a pas été confrrmé par un autre Obfervateur. D'ailleurs, les petites erreurs auxquelles nous avons été quelquefois expofés dans la mefure des angles verticaux, tirent d'autant moins à conféquence, qu'une minute de plus ou de moins dans la hauteur d'un objet, ne produit fouvent pas une feconde de différence dans la réduction d'un angle à l'horizon.

C'eft aufil par cette confidération, que lorfqu'il en̂t fallu trop prolonger notre féjour dans une ftation, nous nous fommes. 
quelquefois difpenfés d'obferver certains angles verticaux, dans les cas où l'on pouvoit y fuppléer par le calcul, \& en les déduifant d'autres angles obfervés ou obfervables dans une autre 1tation.

La frxième Colonne de la Table repréfente donc les angles de hauteur \& de dépreffion, fous lefquets fe voient réciproquement l'un de l'autre les deux Signaux dont la diftance eft exprimée dans la Colonne précédente. Par exemple (Triang: I, lignze 3.) vis-à-vis de la diftance narquée $P C_{9022^{2}, 96}$ à la cinquième Colonne, on trouve dans une accolade (Col.VI.). les nombres $-4^{\mathrm{d}} 20^{\prime} 29^{\prime \prime} \&-I^{\mathrm{d}} \mathrm{I} \mathrm{I} \mathrm{I}^{\prime} 53^{\prime \prime}$ fous cette forme, Oyambaro. $\left|0.63^{\mathrm{d}} 4^{8^{\prime}} 16^{\prime \prime}\right|-3^{\prime \prime} \mid P C .9022,96\left\{\begin{array}{l}P_{.}+4^{\mathrm{d}} 20^{\prime} 29^{\prime \prime} a . \\ C_{0}-1 \text { 1 } 153 \text { a.c. }\end{array}\right.$ ce qui fignifie que d'Oyambaro \& du point $O$, d'où l'on a obfervé l'angle de $63^{\mathrm{d}} 4^{\prime} 8^{\prime}$ I $6^{\prime \prime}-3^{\prime \prime}$ entre les Signaux $P \& C$, diftans de 9022 toifes, on a pris auff la hauteur apparente du Signal $P$ de Pamba-marca de $4^{\mathrm{d}} 20^{\prime} 29^{\prime \prime}$, avec le Quartde-cercle $a$, \& l'abaiffement apparent du point $C$ de I d I I' $53^{\prime \prime}$, en prenant un milieu entre les obfervations des Quarts-decercle $a \& c$. Les quantités affectées du figne + défignent les hauteurs, \& celles qui font précédées du figne - indiquent Jes dépreffions.

Quand, par les raifons rapportées ci-deffus, quelque hatrteur ou quelque dépreffion n'a pas été obfervée d'un certain Signal, on y a fuppléé par le calcul, en la déduifant des obfervations faites aux ftations précédentes ou fuivantes; \& alors, au lieu des lettres $a, b, c, d, c$, qui fervent à défigner les Quarts-de-cercle ( $V_{\text {oy. art. }} I X_{\text {. }}$ ), on a averti par ces lettres cal. que la hauteur ou da dépreffron avoit été calculée. 


\section{A R T I C E XII.}

Explication de la Colonne VII: Hautcurs \& Abaiffemens refpectifs des Signaux.

A chaque angle de hauteur ou de dépreffion, marqué dans Ia Colonne VI, il répond dans la Colonne VII un nombre précédé du figne + ou du figne - Ce nombre exprime la quantité de toifes dont le point obfervé eft plus haut ou plus bas que la ftation de l'Obfervateur, laquelle eft indiquée dans la feconde Colonne. Par exemple, dans le premier Triangle, à la fuite de f'angle de hauteur cotté $O+$ I ' $6^{\prime}$ I $9^{\prime \prime} a . b$ (Colonne VI), on trouve fur la même ligne, \& dans la Colonne VII, le nombre I 26, I I précédé du figne + ; ce qui frgnifie que le point $O$, ou le Signal d'Oyambaro, dont la hauteur apparente a été obfervée du point $C$, eft élevé de $\mathrm{r} 26^{\mathrm{t}}$, I I au deffus du même point $C$, lequel défrgne le Signal de Carabourou, dont le nom eft au commencement de la ligne (Col. II). De même, vis -à - vis de l'angle cotté $C-\mathrm{I}^{\mathrm{d}}{ }_{\mathrm{I}} \mathrm{I}^{\prime} 53^{\prime \prime} a_{1} c($ Col.VI), on retrouve encore dans la Colonne VII le nombre i 26 , i I précédé du figne-; ce qui veut dire que te point $C$, ou le Signal de Carabourou, eft I $26^{\mathrm{t}}$, I I plus bas que celui d'Oyambaro.

II eft évident quiil en eft de même de tout autre nombre qui exprime la différence de hauteur de deux Signaux, \& qu'il doit pareillement, \& par la même raifon, fe rencontrer deux fois "dans la Colonne VII; une fois en hauteur, \& une fois en dépreffion. 
On peut remarquer encore dans cet exemple, que le nombre I 26, I I répond à deux angles différens; favoir, à l'angle de hauteur apparente d'Oyambaro, obfervé à Carabourou, de I 6' I $^{\prime \prime}$, \& à l'angle de dépreffion apparente de Carabourou, obfervé à Oyambaro, de I d I I' $53^{\prime \prime}$. L'inégalité de ces deux angles, \& en général celle des angles de hauteur \& de dépreffion réciproquement obfervés, eft une fuite de la courbure de la Terre, \& de ce que ces angles fe mefurent relativement à l'horizon de l'obfervateur, ou pluftôt à la tangente du lieu de l'obfervation.

Il eft aifé de démontrer, $I^{\circ}$ que l'angle de dépreffion eft toûjours plus grand que l'angle de hauteur. $2^{\circ}$ Que ces deux angles font d'autant plus inégaux, que la diftance entre les deux objets eft plus grande. $3^{\circ}$ Que la différence de ces deux angles, fi elle n'étoit pas un peu diminnuée par ta réfraction, feroit précilément égale à l'angle formé au centre de la Terre (fuppofée fphérique) par les deux rayons qui fe terminent aux poịnts obfervés. $4^{\circ}$ Que ce qui manque à cette différence pour égaler l'angle au centre, eft li fomme des deux réfraçions qui ont altéré les deux angles obfervés. $5^{\circ}$ Que quẹtçuefois deux objets, vûs l'un de l'autre, paroiffent tous deux réciproquement abaiffés fous l'horizon; \& qualors ce n'eft plus la différence, mais la fonme des deux angles de dépreffion obfervés, qui, fauf la réfraction, eft égale à l'angle au centre de la Terre. $6^{\circ}$ Que l'angle vrai de hauteur d'un objet quelconque, eft égal à l'angle de hauteur apparente, augmenté du demi-angle au centre de la Terre, \& diminué de la réfraction : \& réciproquement que l'angle vrai de dépreffion eft égal à l'angle apparent, augmenté de la réfraction, \& diminué 
de langle au centre de la Terre. $7^{\circ}$ Que par conféquent la réfraction qui peut, fans erreur fenfible, être fuppofée égale dans les deux angles, fera corrigée dans le calcul, en prenant pour l'angle vrai, de hauteur, ou de dépreffion, la demifomme des deux angles obfervés. Toute cette théorie eft fondée fur des démonfrations très-fimples, dont je retranche le détail, qui me méneroit trop loin. Elles pourront être fuppléées en partie, par l'infpection de la figure, dans laquelle $B C K$ repréfente l'angle au centre de la Terre, fuppofé PIanche I, formé par le concours des deux rayons $B C, K C$, ou lignes verticales des points $B \& A$; $b a$ l'arc de la furface de la Terre au niveau de la mer, compris entre les mêmes verticales; $A Q L, K M B$, deux arcs concentriques au premier, \& pris au niveau des points $A \& B ; A L, K B$, les cordes de ces arcs; $A E, B F$, les tangentes des rayons $C A, C B ; E A B$ l'an:gle de hauteur apparente du point $B, \& L A B$ l'angle de hauteur vraie du même point $B$, vû de $A ; F B A$ l'angle de dépreffion apparente du point $A$, vû de $B, \& K B A$ l'angle de dépreffion vraie du même point; $C O$ une perpendiculairé tircé du centre $C$ fur la corde $L A$, \& qui partage l'angle $L C A$ au centre de ta Terre en deux parties égales.

\section{A R T I C L E XIV.}

Hauteurs abfoines des Signaux de la Méridieme o des montagnes principales de la Province de Quito.

I L.es hauteurs des Signaux, rapportées dans la Colonne VII de la Table des Triangles, ne font, comme l'indique le titre, que les hauteurs refpectives, ou celles d'un Signal par rapport: 
à un autre; mais on peut, par de fimples additions ou fouf tractions, déduire de ces hauteur's relatives, Ia hauteur abfolue de chaque Sigual au deffus du niveau de la mer ; pourvâ qu'on connoiffe d'abord celle d'un feul Signal. C'eft ainfi que j'ai formé la lifte fuivante de la hauteur abfolue de tous les Signaux de la Méridiemne, en fuppofant que Carabonron, la plus baffe de toutes nos ftations, étoit élevée de 1226 toifes au defius de la furface de la mer; comme je l'avois conclu dès I 740 , par un très-long \& très-fantidieux calcul, fondé fur quelques angles obfervés par M. Bouguer dans l'ifle de l' lnca fur la rivière des E'meraudes, au Nord-oueft de Quito; fur quelques autres que nous avions obfervés enfemble au bourgo du Quinché, à l'Ent de cette même ville, \&̊c.

M. Bonguer, par un calcul femblable, avoit d'abord déduit la hauteur abfolue de Carabonrou, de 12 I 4 toifes. L'élévation de ce point au deflus de l'ifle de l'Inca étoit conclue géométriquement ; mais celle de l'ifle au deffus du nivenu de la mer n'étoit fondée que fur la différence de hauteur du Baromètre, \& fur: l'eftime de la pente moyenne de la rivière des E'merandes. Une autre combinaifon des mêmes élémens, tirée de mes propres. obfervations du Baromètre au bord de la mer, \& des différentes vîteffes de cette rivière, dont javois levé le cours en \736, me fit juger quil y avoit environ douze toifes à ajoûter à la hauteur conclue par $M$. Bonginer, à qui je fis part dans le temps de mes calculs \& de ma détermination. J'ai vû dans les Mémoires de l'Académie de I 744, qu'il s'étoit arrêté au même nonıbre que moi.

Il n'y a aucune hauteur des Signaux de la Table fuivante; qui ne foit le réfultat moyen de deux diverfes déterminations 
lefquelles ne diffèrent communément entr'elles que d'une toife, rarement de trois. Par exemple, la hauteur de $2222^{\mathrm{t}}, 53$ du Signal de Pitchincha, qui a fervi à nos Triangles, eft moyenne entre deux hauteurs du même Signal; l'une de 222 I $^{t}, 44$, conclue par l'angle obfervé à Tanlagoa; l'autre de $2223^{\mathrm{t}}, 62$, déduite de l'angle obfervé à Pamba-marca; \& les hauteurs de chacune de ces deux dernières montagnes ont été pareillement tirées du milieu de deux autres obfervations. Cette méthode, outre qu'elle me fourniffoit une vérification, a dû communément me donner plus de juftefie dans le réfultat : car fi l'une des deux obfervations eft bonne, \& qu'elles diffèrent l'une de l'autre de deux toifes; en prenant le milieu, on n'aura qu'une toife d'erreur à craindre.

L'irrégularité de la réfraction, le défaut d'obfervation réciproque des angles de hauteur \& de dépreffion, \& quelques erreurs accidentelles, ont donné dans une ou deux occafions. jufqu'à cinç toifes de différence. Dans ce cas, comme dans les autres, on a pris le nombre moyen. II eft vrai auffi que jai fait outre cela quelques corrections aux angles verticaux; dans deux ou trois ftations; mais ce n'a été qu'après m'être convaincu par un examen fcrupuleux, qu'elles étoient néceffaires, \& je fuis en état d'en rendre compte. D'ailleurs, ces corrections n'ont jamais paffé une minute : je ne parle point de quelques erreurs manifeftes de 1 o minutes, que jai reconnues \& corrigées.

Aux hauteurs des Signaux, j’ai cru devoir joindre celle des plus hautes montagnes de la province de Quito, qui pourroient bien être aufli les plus hautes montagnes du monde: puiqque le fol qui leur fert de bafe eft communément élevé 


\section{S4 MESURE DES TROIS PREMIERS}

de I 5 à I 6 cens toifes au deffus du niveau de la mer. De là les eaux prennent leur cours vers tous les points de l'horizon, \&. donnent naiffance aux rivières de Guayaquil, des E'meraudes, \& à celle de Napo, qui a long - temps été regardée comme la principale fource du Maranon, ou du fleuve des Amazones.

Je ne donne ici que la lifte des montagnes les plus remarquables de la province de Quito, de celles qui offrent le fpectacle fingulier de la neige dont leur formmet eft toûjours couvert, \& au milieu de laquelle on voit, dans quelques-unes, les flanmes s'ouvrir un paffage. Il ne neige jamais à Quito, dont le fol eft à I 560 toifes de hauteur perpendiculaire fur la furface de la mer; deux ou trois cens toifes plus haut, Ia neige couvre quelquefois la terre, mais cette neige fe fond bien-tôt; \& ce n'eft guère qu'à 2440 toifes de hauteur qu'elle fe conferve fans jamais fe fondre: c'eft ce que nous avons conftamment remarqué aux environs de l'E'quateur.

Planche II, La Figure 2 de la feconde Planche eft une coupe du terrein de fig. 2.

la Méridienne fur fa longueur de plus de trois degrés. Malgré la petiteffe de l'échelle, la courbure de l'arc du Méridien au niveau de la mer y eft fenfuble fur cette diftance, \& fe diftingue de la corde du même arc laquelle eft auffr tracée. La hauteur de tous nos Signaux, celle des montagnes, celle de nos deux obfervatoires aux deux extrémités de la Méridienne, \& celle du fol des trois principales villes de Ia province, Quito, Cuenca, \& Riobamba, y font repréfentées en proportion avec leurs diftances en latitude, \& fur la même échelle que la Carte des Tiriangles de la Méridienne qu'on voit immédiatement au deffuis. L'afpect des montagnes eft conforme dans ce profil à celui qu'elles offrent en effet. $L_{\text {an }}$ difiérence de leurs hauteurs fe 


\section{DEGRÉS DU MERIDIEN.}

manifefte à la vîe dans le deftein, \& de plus on l'a exprimée en toifes. Pour éviter la confufion, les noms des montagnes font écrits au deffus de l'arc, \& ceux des Signaux le font au deffous; chacun vis-à-vis d'une ligne verticale ponctice qui répond au point défrgné. La ligne horizontale ponctuée marque la hauteur du niveau de I 440 toifes, à laquelle la neige ne fond plus. On en̂t pû divifer l'arc du Méridien, au niveau de Ia furface de la mer, de minute en minute, comme dans la carte des Triangles; on s'eft contenté dans le profil, de marquer l'Équateur \& fes Parallèles de degré en degré.

La carte \& le profil ont été réduits d'après un deffein d'une échelle quadruple, fait fous mes yeux dès I 740 . Je réferve pour une autre occafion une carte de la Province de Quito, dreflée avec foin par $M$. Verguin; à lacquelle j’ai contribué pour ma part, en domnant le détail de 40 lieues de Côtes, le cours de la Rivière des E'meraudes, \& plus de 400 relévemens. $T A B L E$ de la hauteur des Signaux de la Méridienne de Quito au deffus du niveau de la mer.

Les noms Efpagnols font en caractère romain.

Carabourou, terme nord de la première Bafe .. 1 1226,00 Oyambaro, terme fud... $1352, \mathbf{1} 1$ la Tola de Cotchefqui . I 490, 84 Pamba-inarca ...2 $2109,80$. Goapoulo (le.Sign,deM.Godin) 1 54 I, 85 Goamani, idem . . 2080, 42 Tanlagoa .... I 743, 37 Pitchincha (le Signal) . 2222, 53 Schangailli..... 1405, 53 El Coraçon (le Signal) . 22 12, 18 Pouca-ouaïcou, Signal fur Koto-pacf. . . 2264, 47
Milin toifes. Milin..... 1793,82 Papa-ourcou . . . 1 828,39 Ouango-taffin ...22086, 29 Tchoulapou. .... 1952, 58 Hivicatfou. ... I 575,09 Chitchitchoco.... 1824, 22 Moulmoul....22006,09 Yoalata ou Goayama 2243,75 Ilinal...... 1941,77 Dolomboc ou Siça-pongo 2098, 52 Nabouço. . . I715,98. Amoula ..... 1790, 38 Zagroum, ..... $1813,8 \mathrm{z}$ 


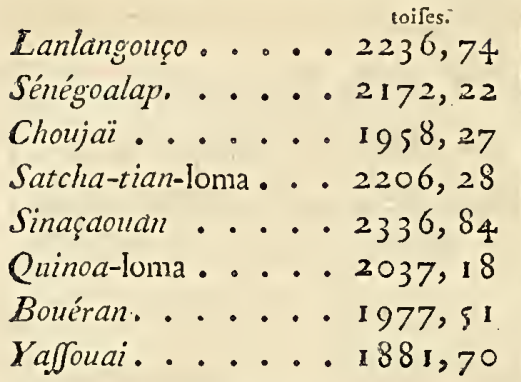

Coines: Cahouapata ....1819,97

Borma...... 1614,06 Pongin ..... 1482,69

Pillatchiquir. . . I 1728,94 Ailpa-roupachica ... I 587,75 Ouctoua-tarqui, terme

nord dela fecondeBafẹ. I 365,34 Chinan, terme fud . 1411,37.

HAUt EUR du Sol de quelques lieux de la Province de Quito.

Sol de Quito, grande place ........ 1462 toiles:

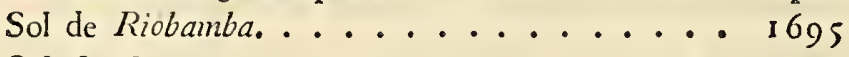

Sol de Cuenca.........................

HAUT EU R des montagnes les plus remarquables de la Province de Quito, dont les fonmets font couverts de neige, \& dont la plufpart ont été ou font actuellement Volcans.

On a défigné ta pofition des deux premières montagnes, parce qu'elle n'eft pas comprife dans la carte des Triangles, ni dans le profil de la Planche II.

Cota-catché, à 33000 toifes au nord de Quito..... soiles:

Cayambé-ourcou, fous l'E'quateur même, 34000 toifes à l'Eft de Quito .............. 3030

Pitchincha, Volcan en I 539 , I $577 \&$ I 660. Son fommet

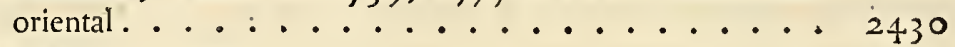

Anti-fana, Volcan en I $590 \ldots 3020$

El Coraçon, la plus grande hauteur connue où l'on ait monté . 2470

Sinchoulagoa, Volcan en 1660 , communiquant avec Pit-

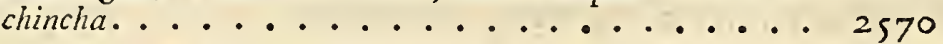

Iliniça, préfumé Volcan ........... 2717

Koto-pacfi, Volcan en 1533, 1742 \& $1744 \ldots . . .2950$

Chimbo-raşo, Volcan. (on ignore l'époque de fon éruption). 3220

Cargavi-raço, Volcan écroulé en 1698 . . . . 2450

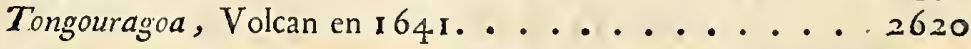

El Altar; l'une des montagnes appelées Coillanès . . . . 2730

Snngä, Volcan continuellement enflammé depuis $1728 \ldots \ldots .2680$ 


\section{A R T I C L E XV.}

Explication de la Colonne VIII: De la réduction des angles obfervés en differens plans, à l'horizon.

DEUX chânes de hautes montagnes, difpofées à peu près parallèlement, à quelques lieues de dîftance l'une de l'autre, nous ont fourni la plufpart des points d'appui de nos Triangles; c'étoit tantôt fur le fommet, tantôt fur le penchant de ces montagnes, \& quelquefois dans le vallon qui les féparoit, que nous placions nos Signaux; felon que la nature du terrein, \& la meilleure difpofition des Triangles l'exigeoit. II y a eu quelquefois jufquà 900 ou r 000 toiles de différence de hauteur entre deux Signaux voifms.

Pour déduire de ces Triangles, obfervés dans des plans fi diverfement inclinés, la longueur de la Méridienne; il a fallu commencer par réduire chaque Triangle à un plan horizontal, $\&$ les rapporter tous à un même niveau. Cette réduction peut fe faire par la Trigonométrie rectiligne, \& par la Trigonométrie fphérique: je me fuis fervi de celle-ci, quii n’a pas befoin pour cette opération de confidérer l'amplitude de l'arc itinéraire entre l'obfervateur \& le point obfervé; \& qui, par cette raifon, procède dans le calcul des angles réduits à l'horizon, d'une manière plus limple, \& plus indépendante de toute fuppofition anticipée de la figure de la Terre.

Rapporter à un plan horizontal, l'angle obfervé entre deux wbjets, dans un plan incliné; c'eft la même chofe que chercher l'angle que comprendroient deux plans verticaux, qu'on feroit paffer par ces deux objets, \& par le zénith 
de l'obfervateur. Or dans le Triangle fphérique que forment ces trois points, les trois côtés font donnés; l'un entre les deux objets, par l'obfervation de l'angle de pofition; les deux autres, par la diftance des mêmes objets au zénith; c'eft-àdire, par le complément de la hauteur obfervée de chaque objet. Par ces trois côtés connus, on aura de quoi conclurre l'angle au zénith, qui eft le même que l'angle horizontal cherché. C'eft de cette méthode que je me fuis fervi pour la réduction de tous les angles qui compofent la Colonne VIII de la Table, dans laquelle chaque angle réduit à I'horizon eft placé vis-à - vis de l'angle obfervé qui lui répond dans la feconde Colonne. Tous les ééémens de cette réduction fe trouvent auffi fur la même ligne dans les Colonnes précédentes.

Par exemple: on a vî que ilangle $O$ (Col. I, Triang. I), au Signal d'Oyambaro (Col. II), a été obfervé de $63^{\mathrm{d}} 48^{\prime}$ I $6^{\prime \prime}$ (Col. Ill, fig. 3), - $3^{\prime \prime}($ Col. IV ), entre les points $P \& C$ $(C o l . V)$, ceeft-à-dire, entre les Signaux de Pamba-marca \& de Carabourou, diftans l'un de l'autre de $9022^{\mathrm{t}}, 96$. Outre cela, on voit $(C o l . V I)$ que le point $P$, toûjours vû de $O$, a parı élevé de $4^{\mathrm{d}} 20^{\prime} 29^{\prime \prime}$ fur l'horizon, avec le Quart-de-cercle $a$; \& que le point $C$ a paru abaiffé de I $^{\mathrm{d}}$ I I $^{\prime} 53^{\prime \prime}$ avec les Quarts-de-cercle $a \& c$ : d'où il fuit que les diftances apparentes des points $P \& C$ au zénith d'Oyambaro font de $85^{\mathrm{d}}$ $39^{\prime} 3 I^{\prime \prime}, \&$ de $9^{I^{d}}$ I I $^{\prime} 53^{\prime \prime}$. Or ces 'deux diftances au zénith, \& P'arc obfervé entre les deux points $P \& C$, font les trois côtés connus d'un Triangle fphérique, dont la réfolution fera trouver l'angle au zénith de $63^{\mathrm{d}} 3^{6}{ }^{\prime} \mathrm{o}^{\prime \prime}$ (Col. VIII.) Cet angie eft vifiblement le même que l'angle $O$ réduit à 
Thorizon; puifque celui-ci eft la fection horizontale de deux plans verticaux qui pafferoient par l'œil de l'obfervateur, \& par les deux points obfervés $P \& C$.

La fomme des trois angles réduits s'ett trouvée ordinairement plus grande que I 80 degrés, d'une, de deux, ou de trois fecondes: on en domnera la raifon dans l'explication de la Colonne fuivante. On a retranché cet excès proportionnellement fur les trois angles, en rédui fant les fommes à deux droits.

\section{A R T I C L E XVI.}

Explication de la Colonne IX: Longueur des côtés horizontaux, réduits au niveau de Carabourou.

LA neuvième Colonne de la Table repréfente la longueur des côtés de tous les Triangles, réduits au niveau de Carabourou, le plus bas de nos Signaux. Voici le procédé que jai fuivi pour cette réduction.

Mes angles réduits à l'horizon me fourniffoient une nouvelle fuite de Triangles horizontaux, qui pouvoient être rapportés à un niveau quelconque. La longueur des côtés, proportionnels dans tous ces Triangles, dépendoit de celle quion attribueroit au premier côté. J'ai pris pour ce premier côté, \& pour fondement du calcul de cette nouvelle fuite de Triangles, ta Bafe conclue de la mefure actuelle, \& déjà réduite (art. I) au niveau de Carabourou; \& j'en ai déduit la longueur de tous les côtés horizontaux pour ce même niveau, telle qu'elie eût été conclue des angles obfervés, fi tout le terrein de la Méridienne eût été parfaitement uni, \& à la haureur du terme feptentrional de notre Bafe. De cette manière, 
jai été difpenfé de réduire chaque côté au niveau de l'une de fes deux extrémités, \& de rapporter enfuite à une même hauteur tous ces côtés réduits, un à un, à différens niveaux.

J'ai choifı le niveau de Carabourou, par préférence à celui de tout autre Signal; parce que ce lieu étoit Ja plus baffè de toutes nos ftations, \& en même temps un des deux termes de notre première Bafe. Une fimple opération fuffit pour réduiire Ja tongueur du degré, prife à la hauteur de Caraboltrou, à fa vraie longueur, au niveau de la furface de la mer.

J'ai fuppofé dans mon calcul, que les trois angles d'un Triangle rectiligne réduits à l'horizon, formoient un autre Triangle rectifigne; ce qui n'eft pas vrai en rigueur mathématique. II faut en dire la raifon, \& faire voir qu'il n'y a point d'erreur fenfible à craindre des fuites de cette fuppofition.

Réduire un angle à l'horizon, c'eft le réduire à un plan horizontal tangent à la furface de la Terre dans le lieu de l'obfervation. Trois angles obfervés en trois points d'une fphère; \& réduits chacun à lewr horizon, ou à̀ leur plan tangent, appartiennent à trois plans différens; ils ne peuvent donc formes: un Triangle rectiligne, qui eft effentiellement dans un feul plan.

Mais les fommets de ces angles, les trois points par lefquels les trois plans touchent ta fphère, font dans la furface de la fphère, \& y forment un Triangle Sphérique, dont une des propriétés. eft que la fomme de fes trois angles eft néceffairement plus grande que deux droits; auffi dans mes calculs In fomme des trois angles réduits a $-\mathrm{t}$ - elle prefque toûjours. excédé 180 degrés. Cet excès a été rarement à trois fecondes; quantité qui pourroit être négligée, puifquon n'ẹn peut guère répondre, dans la mefure d'un angle avec un inftrument de 
trois pieds de rayon; cependant au lieu de n'en tenir aucun compte, elle a toûjours été répartie fur les trois angles, dans la réduction qui a été faite de leur fonme totale à deux angles droits.

Par cette réduction; on a en quelque forte fubftitué au Triangle fphérique, formé par trois arcs de grand cercle, le Triangle rectiligne formé par les trois cordes de ces mêmes arcs; \& au lieu de réfoudre les Triangles réduits à l'horizon comme curvilignes, en prenant pour premier côté connu Ia Bafe réduite en arc (art. I); on a pris pour premier côté, la corde de cette même Bafe, \& on en a conclu par la Trigonométrie rectiligne les côtés fuivans; c'eft-à-dire, les cordes des autres arcs \& non les arcs mêmes. En procédant ainfi on a néceffairement fuppofé que les cordes des petits arcs. avoient entr'elles le même rapport que les arcs; mais on peut voir facilement combien cette fuppofition, comme celle de piendre les cordes pour les arcs, tire peu à conféquence dans le cas prélent. Pour cela, il fuffit de remarquer que le plus long côté de nos Triangles n'eft que de 2 I 000 toifes; que réduit en arc de grand cercle, il ne vaut que 22 minutes. quelques fecondes, \& qu'il n'a qu'un pied de longueur plus: que fa corde.

La Trigonométrie 'Sphérique a paru plus commode pour réduire à Phorizon les angles de pofition obfervés; mais il y: eût eu beaucoup plus de travail, \& il n'y auroit rien eu à gagner du côté de l'exactitude, à fe fervir de ce moyen pour chercher: la valeur des côtés des Triangles réduits, en les confidérant comme des arcs de cercles: je les ai donc pris fans frupule. pour deș lignes droites Les faufles fuppofitions ne font a: 
craindre dans les calculs mathématiques, que lor'qu'elles ' $s^{\prime} y^{\prime}$ gliffent faus quion s'en aperçoive, ou lorfqu'on les admet fans en prévoir les conféquences: employées à propos, elles fervent à faciliter les calculs, fans induire en erreur.

Telle eft la fuppofition de la fphéricité de la Terre, fur laquelle tous les calculs par la Trigonométrie fphérique font fondés. Cette même fuppofition ne peut manquer de revenir fouvent dans cet Ouvrage. Mais fi un Triangle fphérique de 20000 toifes de côté peut, comme on vient de voir, être pris fans conféquence dans le cas préfent 'pour un Triangle rectiligne formé par fes trois cordes; à plus forte raifon un petit arc d'un fphéroïde très-peu aplati, tel qu’eft la Terre; fe confond-il avec l'arc correfpondant de Ia fphère infcrite ou circonfcrite.

\section{A R T I C L E XVII.}

Explication de la Colonne X: Direction des côtés des Triangles par irapport à la ligne Méridienne.

LA longueur connue de tous les côtés des Triangles de la Méridienne, ni même la longueur totale de la chaine qu'ils forment, ne penvent fervir à conclurre la longueur de la Méridienne, fi l'on ne connoît la pofition de tous les Triangles par rapport à cette ligne; c'eft-à-dire', la déclinaifon de leurs côtés, ou l'angle qu'ils font avec la ligne nord \& fud. Les angles réduits à l'horizon, domnent la direction refpective d'un. côté par rapport à l'autre : il fuffiroit donc en rigueur d'avoir par obfervation la déclinaifon d'un des côtés par rapport aux 
régions du monde, pour conclurre celles cie tous les autres.

Nous avons commencé par bien nous affurer de li déclimaîfon de notre première Bafe à l'égard de la Méridienne, en obfervant à plufieurs reprifes à Oyambaro, terme auftual de cette Bafe, l'angle compris entre le foleil levant où couchant, \& les Signaux de Tanlagoa \& de Pamba-marca, les plus voifins de la Bafe; \& par le moyen réfultat de trois ou quatre obfervations différentes, nous avons conclu que la ligne tirée d'Oyambaro à Carabourou, c'eft-à-dire, du terme auftral au terme boréal de la Bafe d'Yarouqui, déclinoit du nord à l'oueft de $19^{d} 25^{\prime} \&$ quelques fecondes.

Cette direction une fois fixée fuffit, comme on voit, pour donner celle de tous les côtés des Triangles, par la fimple addition ou fouftraction des angles horizontaux, compris entre ce premier côté \& les fuivans fucceffivement. C'eft Ia lifte de toutes ces directions, ainfi conclues par rapport à Ia première obfervée, qui compofe la neuvième Colonne. Mais comme les petites erreurs, quil n'eft pas poffible de prévenir dans la mefure des angles, pourroient, en s'accumulant, caufer après une longue fuite de Triangles, une erreur confidérable dans la direction des côtés; il étoit à propos de vérifier de temps en temps, par de nouvelles obfervations aftronomiques, la pofition de quelques côtés de Triangles par rapport à la Méridienne. C'eft à quoi nous avons eu grande attention. Nous avons profité des occafions les plus favorables, pour obferver dans le cours de notre travail l'angle entre le Soleil levant ou couchánt, \& divers côtés de Triangles: \& nous avons eu la fatisfaction de trouver que l'oblervation répondoit au calcul, quelquefois fans différence fenfible, 


\section{MESURE DES TROIS PREMTIERS}

- quelquefois à une minute près, tantôt en plus, tantôt en moins? ce qui n'eft de nulfe confidération en pareille rencontre, furtout fil'on fait attention que cette différence eft la fomme des erreurs qui peuvent avoir été commifes, d'une part dans une longue fuite d'angles de pofition obfervés, \& de l'autre dans une obfervation d'azimuth, très-délicate par elle-même, trèscompliquée par le nombre d'élémens qui y entrent, laquelle exige le concours de deux obfervateurs, \& enfin qui n’a pî le plus fouvent fe faire quà la hâte, fans Pendule, avec beaucoup d'incommodité \& de froid, fur le fommet d'une montagne, d'où l'on étoit toûjours preffé de partir.

Nous avons fait enfemble, ou féparément, 18 ou 20 de ces obfervations d'azinuth dans la longueur de la Méridienne: Pour ce qui me regarde, j'en ai obfervé douze, foit feul, foit avec $M$. Bougucr, quelquefois deux ou trois dans la même ftation; \& alors j’ai pris un milieu. J'ai calculé toutes ces obfervations plufieurs fois; je n'ai pas fait d'ufage de deux ou trois faites avec précipitation, ou lorfque le foleil étoit fort-ondulant, par une réfraction irrégulière. Toutes les autres font employées dans la Table: on trouvera leur réfultat au deffous de celui que donne la fuite des angles hórizontaux.

Lorfqu'on veut comparer la direction obfervée d'un côté dè Triangle, à la direction conclue; on eft ordinairement obligé de faire une réduction, à caufe de la convergence des Méridiens. Cette réduction, quoique moins néceffaire aux environs de l'E'quateur, où les Méridiens font fenfiblement parallèles, n'a cependant pas été négligée. J'ajoûte qu'elle ne devoit pas lềtre; puifque fur la longueur des trois degrés que nous avons mefurés la direction d'un côté de Triangle, conclue par l'addition fucceffivg 
fucceflive des angles horizontaux, ne laiffe pas de différer de près de deux minutes de la direction vraie de ce même côté, lorfqu'on a égard à la convergence des Méridiens.

$P$ repréfente le Pole Sud; $Q$ Quito; $Q P$ le Méridien de cette ville, auquel on veut rapporter la direction obfervée d'un côté $O S$ d'un Triangle quelconque. $O P$ eft le Méridien de Planche I, l'Oblervateur; $T O P$ la tangente de ce même Méridien, Iaquelle en repréfente la direction au point $O$. Par la fuite des Triangles horizontaux, qu'on a confidérés comme dans un même plan, on a trouvé la valeur de l'angle $S O R$, entre le côté $S O \&$ la ligne $O R$ parallè̀e à $Q P, M e ́ r i d i e n$ de $Q$ uito , \& qu'on a prife pour le Méridien du point $O$. Mais l'angle véritable, que forme le côté $S O$ avec le Méridien du point $O$, eft l'angle $S O T$, compris entre la tangente $O T \&$ la ligne $O R$ parallèle à $Q P$. C'eft cet angle quiil a fallu chercher à chaque obfervation d'azimuth; pour pouvoir comparer la direction oblervée par l'azimuth, à la direction conclue par liaddition fucceffive des angles horizontaux, depuis la première direction obfervée.

\section{A R T I C L E XVIII.}

Explication des Colonnes XI \& XII de la Table:

$$
\begin{gathered}
\text { Diftances entre les Méridiens \& les Parallèles } \\
\text { des Signaux. }
\end{gathered}
$$

LA Iongueur \& Ia direction d'un côté quelconque de Triangle étant connues, on peut, de l'une de fes extrémités, mener une perpendiculaire, de l'autre une parallè̀e à la 
Méridienne, \& former ainfı un Triangle rectangle, dont ce côté fera l'hypothénufe, \& dont on connoîtra les trois angles. II fera donc aifé de conclurre la longueur des deux autres côtés qui comprennent l'angle droit.

Ce font cés côtés, ou les diftances entre les Parallèles \& les Méridiens des Signaux de chaque Triangle; ou autrement . ce font les différences en latitude \& en longitude entre les Signaux, réduites en toifes, qui rempliffent les Colonnes XI \& XII de la Table.

Des différentes combinaifons des nombres des Colonnes XI \& XII, qui peuvent donner la diftance entre les Signaux de Cotchefqui \& de Tarqui, tant en latitude qu'en longitude, Ja plis fimple fe fera par l'addition \& la fouftraction des nombres marqués dans ces Colonnes en plus petits caractères.

\section{A R T I C LE X IX.}

Détermination des points des Triangles de la Méridienne à l'égard de Quito.

Our t o étant non feulement le lieu le plus confidérable dans le pays où nous avons opéré, mais une des plus grandes villes de l'Amérique méridionale, cette ville fe trouvant fituée vers le milieu de l'efpace occupé par nos Triangles d'occident en orient; fa latitude ayant d'ailleurs été fixée à

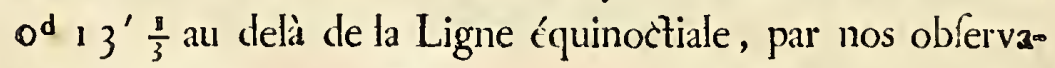
tions des Solftices de Décembre 1736 \& Juin 1737; \& F. longitude pouvant l'être exactement par un grand nombre d'imumerfons \& d'émerfions obfervées des fatellites de Jupites: 
rout a concouru pour me déterminer à rapporter nos mefures au Méridien de Quito. J'ai choifi, par préférence, celui de la Tour de l'Églife des Religieux de la Mercy, par la raifon que ce point, d'ailleurs voifm de la grande Place \& du centre de la ville (Voyez le plan de Quito), a été déterminé par obfervation, \& lié immédiatement à nos Triangles.

C'eft par cette raifon que je joins ici une Table, où l'on trouvera la pofition de tous les Signaux à l'égard du Méridien de la Tour de la Mercy de Quito, \& de la perpendiculaire à ce Méridien. Cette perpendiculaire eft fr voifine de l'Équateur, qu'elle. ne diffère pas fenfiblement d'un Parallèle à ce cercle. La Table eft divifée en deux parties, l'une pour les Signaux occidentaux, l'autre pour les orientaux; l'une $\&$ l'autrè donnent la même diftance entre les Signaux extrêmes de la Méridienne, Cotchefqui \& Tarqui: cette Table eft formée par de fimples additions ou fouftractions des nombres des Colonnes XI \& XII de la Table des Triangles.

Pour lier Quitoà nos Triangles, j'avois obfervé avec M. Bougucr divers angles à Pitchincha, à la maifon de Quito, qui a fervi d'obfervatoire pour l'obliquité de l'E'cliptique, \& fur une petite montagne voifine de la ville; mais ce n'eft qu'en s'enfonçant dans un labyrinthe de calculs qu'on peut déduire de ces angles la pofition de Quito. J'ai trouvé beaucoup plus court \& plus fimple de me fervir d'un Triangle de M. Godin, lequel fe ternine à la Tour même de la Mcrcy de Quito , \& dont j’ai obfervé deux angles. Cela m’a engagé à réfoudre trois Triangles de plus, \& à les ajoûter à ma Table : ce font ceux de la dernière feuille; ils font, comme les auxiliaires, diftingués par des lignes ponctuées. 
68 MESURE DES TROIS PREMIERS

$T A B L E$ des diftances des Signaux à la Méridienne, \& à la réduites au niveau de Carabourou,

Par les côtés occidentaux des Triangles.

Diftances à la Perpenáiculaire de Quito.

Diftances à la Méridienne.

Quito (Tour de la Mercy de)

toires.

roifes.

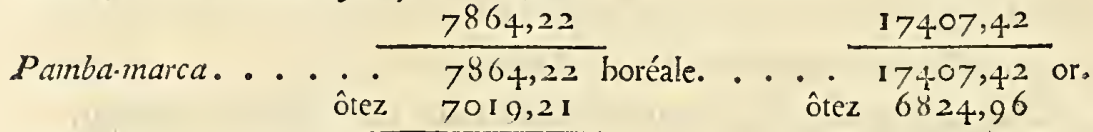

Oyambaro...... 845,01 bor. ....

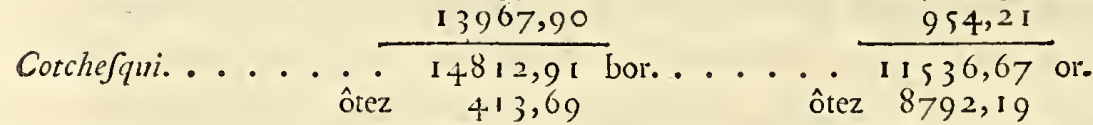

Tanlagoa. ...... $1+399,22$ hor. .... 2744,48 or. ôtez I I 645,24 à fouftraire de 5015,14

Pitchincha ..... 2753,48 bor. . . . 2270,66 cce. à fouftraire de 20366,10

El Coraçon . .... 17612,12 auftrale. ... $\frac{5+17,73}{7688,39}$ occ. 18853,11

$3+81,55$

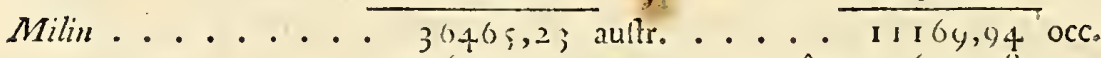

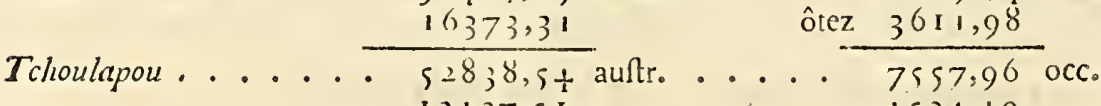

Chitchitchoco ......... $\frac{13127,51}{95966,05 \text { auftr. ... }}$

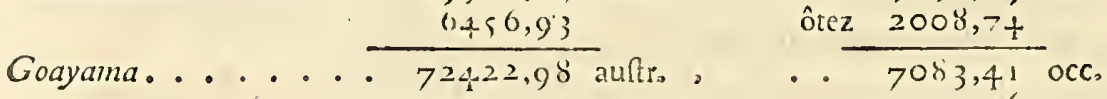

Dolomboc. . . . . . . . . $\frac{121+1,14}{8+564,12 \text { auftr. . }}$

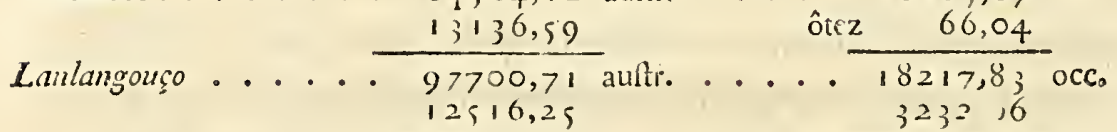

Choujaï......

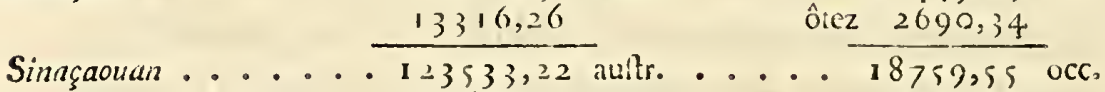

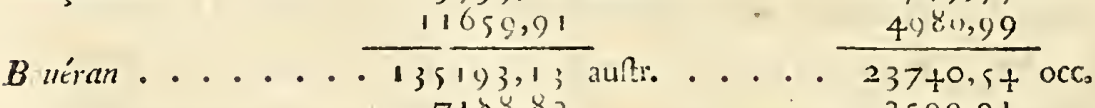

Cahourapata. .......... $\frac{7188,82}{26340,45 \text { ccc. }}$

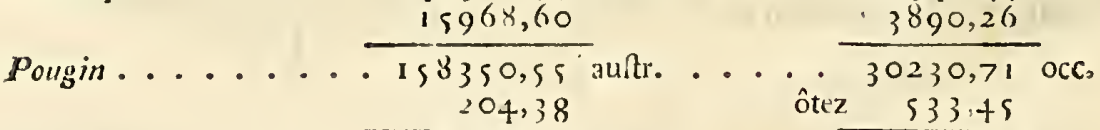

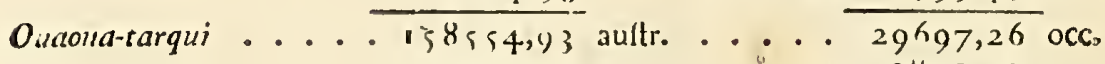

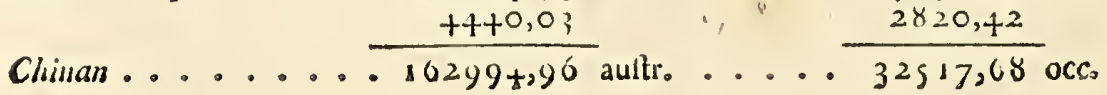


Perpendiculaire fur la Méridienne de la Tour de la Mercy de Quito, le plus bas de tous les Signaux.

Par les côtés orientaux des Triangles.

Diftances à la Perpendiculaire de Quito.

Quito ( Tour de la Mercy de)

Pamba-marca ..... à foultraire d
Difances à la Méridieme.

toires.

0,00

7864,22 boréale.... I 7407,42 orientale.

ôtez 10578,08

Scliangailli ..... 6841,62 aultrale. ... 6829,34 or.

Pouca-ouaícou. ........ ôtez $\frac{18815,05}{25656,67 \text { auftr. ... }}$ signal fur Koto-pacfi. $\quad 3433,82$ a fouftraire de 3526,15

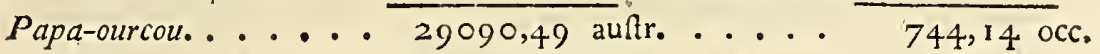

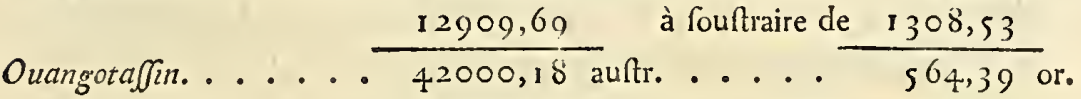

Hivicatfou ......... ôtez $\frac{13721,57}{55721,75 \text { auft. ... }}$

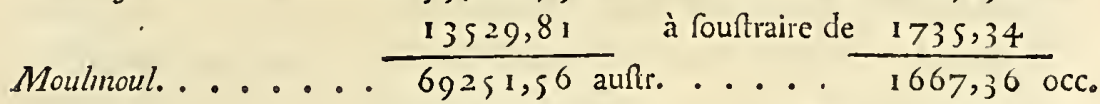

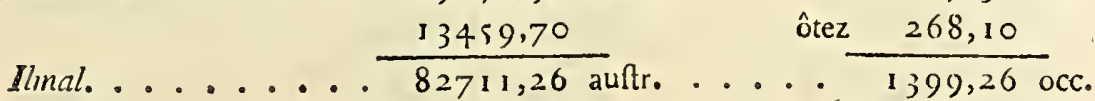

Zagroum............ $\frac{12908,67}{95619,93 \text { auft. ... }}$

Sénégóalap........... $\frac{10240,11}{105860,04 \text { auft. ... }}$

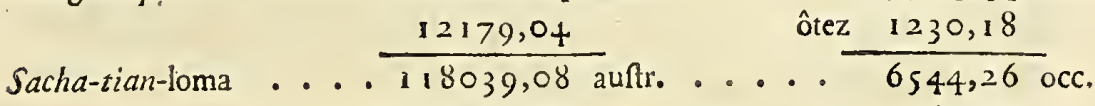

Quinoa-lona........... $\frac{10739,78}{128778,80 \text { auftr. ... }}$

$\frac{11613,74}{140392,70}+\frac{4260,56}{12467,48}$

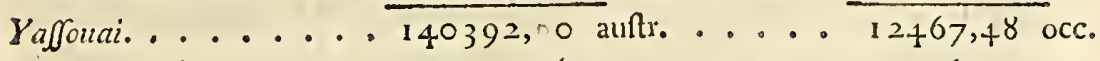

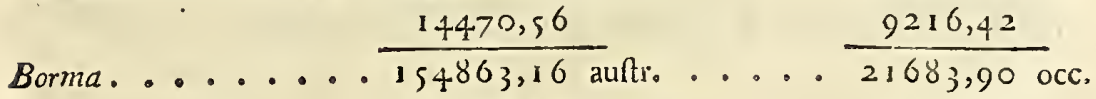

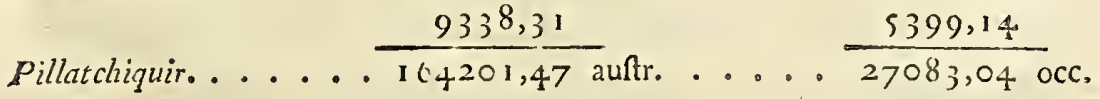

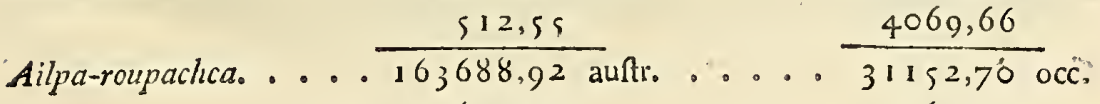

Chinan......... $\frac{693,95}{162994,97} \quad \frac{1364,92}{32517,62 \text { ocs. }}$

L iij. 
En ajoûtant les diftances des Signaux de Cotchefqui \& de Chinan à la Perpendiculaire de Quito, tirées de la Table précédente, on aura la longueur calculée de la Méridiemne, entre les deux Signaux fitués à fes deux extrémités, \& voiíns des obfervatoires de Cotche fqui $\&$ de Tarqui, où ont été faites les obfervations aftronomiques de l'amplitude de l'arc du Méridien.

Diftance feptentrionale de la Perpendiculaire au Méridien de la Tour de la Mercy de Quito, au Signal de Cotchefqui, tirée de la Table précédente, (côtés occidentaux des Triangles $) \ldots \ldots \ldots \ldots \ldots$ I 48 I 2 ,9 I

Diftance auftrale de la même Perpendiculaire, au Signal de Chinan près Tarqui, tircé de la Table précédente, (côtés orientaux des Triangles ). . . . . 162994,96

Somme ou diftance parallèle à la Méridienne entre les Signaux de Cotchefqui \& de Tarqui .......... 177807,87

Il y a plufieurs réductions à faire à cette mefure, pour en tirer la longueur de l’arc du Méridien, intercepté entre les obfervatoires de Cotchefqui \& de Tarqui. Cette difcuffion fera le fujet des articles fuivans.

II faut d'abord examiner fi cette diftance, conclue par un calcul fondé fur la mefure actuelle de notre première Bafe dans ta plaine d'Yarouqui, s'accorde avec la mefure actuelle de notre feconde Bafe dans la plaine de Tarqui. 


\section{A R T I C.LE X X. \\ Mefure de la Bafe de Tarqui.}

LA plaine de Tarqui, à cinq lieues de Cuenca, vers le Sud, à l'extrémité auftrale de nos trois degrés mefurés du Méridien, fembloit nous inviter à la mefure actuelle d'une nouvelle Bale, pour vérifier par fon moyen, après une fuite de Triangles longue de 80 lieues, la longueur calculée de leurs côtés. C'eft le jugement que j’avois porté de cette plaine au premier afpect, en la traverfant plus de deux ans auparavant. lors de mon voyage à Lima en 1737 ; \& dès ce temps je l'avois indiquée à $\mathrm{M}^{\text {rs }}$ Godin \& Bouguer, comme propre à cet ufage. Cependant, quoique très-unie, elle ne laiffe pas d'aroir quarante-huit toifes de pente fur deux lieues; mais ce n'eft que le tiers de la pente de la Bafe d'Yarouqui, comme on peut le voir par la comparaifon des deux profils, que repréfente la planche $I$.

A Tarqui, la pente n'eft fenfible que vers l'extrémité nord Planche I, fig. $1 \& 2$ 。 de. la plaine, où elle eft de 34 toifes fur 660 : il y a même en un endroit un faut ou talus très-roide de plufieurs toifes de haut; mais le refte de la plaine eft fort uni, fur-tout le milieu, qui forme une prairie où ferpente une petite rivière. Le terrein $y$ a $f i$ peu de pente, qu'il y a plufieurs flaques d'eau dormante, entrautres une de plus de 250 toifes de longueur dans les temps les plus fecs, \& que nous traverfâmes dans toute fa longueur en mefurant notre Bafe. Il n'y avoit alors guère plus d'un pied d'eau; \& on n'enfonçoit pas jufqu'au genou dans l'elzdroit le plus profond. Nous fûmes dilpenfés, dans ce trajet, de faire ufage du niveau; les perches, avec lefquuelles nous. 
opérions, flottoient fur l'eau; des piquets, ou les rofeaux mêmes du marais, nous fervoient à les affujétii:

Nous mefurâmes la Bafe de Tarqui au mois d'Août I 739 ; M. Bouguer d'un côté, fecondé de Don Antoine de Ulloa, \& moi de l'autre, aidé de M. Verguin. Nous avions, de part \& d'autre, une large règle de fer, fur laquelle étoit marquée exactement la longueur de la Toife de Paris, prife avec un compas à verge fur la Toife de fer que nous avions apportée de France. Par la vérification qui fut faite entre M. Bouguler, M. Verguin \& moi, de la règle qui m'étoit échûe en partage, elle fe trouva plus fongue que celle qui avoit fervi à M. Bouguer d'une quantité que nous évaluâmes, par différens moyens, \& en prenant le milieu de nos eftimes, à $\frac{1}{27}$ de ligne; ce qui avoit dû me faire compter une ligne de moins que lui, fur chaque 27 toifes; ou I pied 3 pouces 9 lignes fur la longueur totale de Ia Bafe: en effet, je la trouvai plus longue que M. Bouguer de I pied 5 pouces 5 lignes; ce qui ne diffère du nombre précédent que d'un pouce huit lignes par excès. Par une autre comparaifon de nos Toifes de fer; faite quelques jours après, entre M. Bonguter, Don Antoine de Ulloa \& moi, ma mefure particulière de la Bafe, au lieu d'être plus longue de I pouce 8 lignes que celle de M. Bouguer, fe trouva de deux pouces quatre lignes plus courte; \& en prenant le milieu des deux comparaifons, nos deux mefures actuelles ne différoient que de 4 lignes.

Outre les angles obfervés de hauteur \& de dépreffion des différens points, où linclinaifon du terrein changeoit fenfiblement, je:ss foin, en mefurant cette Bafe, de tenir une note de la hauteur des à-plombs, quand il falloit haufer te niveau des 
des perches qui fervoient à mefurer"; ce qui m'a donné deux moyens au lieu d'un, pour tracer le profil que repréfente la figure.

Je prends la mefure actuelle de la Bafe de Tarqui par Planche I, échelons horizontaux à différens niveaux, \& moyenne entre celle de M. Bouguer \& la mienne, de . . . 5259t,20

De Ouaoua-tarqui, terme nord de cette Bafe, au point $U$, on a obfervé la hauteur apparente du point $X$ à Chinan, terme fud de la même Bafe, de..................... od $27^{\prime} 40^{\prime \prime}$

De $X$, terme fud, on a obfervé Ia dépreffion $\operatorname{de} U$, terme nord. ............................ 3230

La différence des deux angles eft ....... 450

L'angle au centre de la Terre, entre les deux verticales aux extrémités d'un arc de $\$ 259$ toifes, eft de. .

Différence.........................

Demi-différence $=$ réfraction pour clraque angle $*$.

De $U$, terme nord, Ie point $A$, éloigné de 200 toifes, a paru bas de ...........

Dù point $G$, ̀̀ I 759 toifes du terme fud $X$, ce point a paru haut de................ I Ix 30

Du même point $G$ de dépreffion apparente de $U$, terme nord. ........................ ro 56

Par ces angles \& par les différences de niveaux obfẹvées, j’ai concla l'abaiffement des points fuivans au deffous de $X$, terme fud
$U \ldots . .44^{\text {zoifes }} \mathrm{s}^{\text {pieds. }}$
A.... $48 \quad 2$
G... $37 \quad 3$
H.... $39 \quad 3$

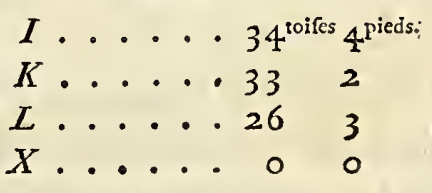

De toutes ces Données, \& par un calcul femblable à celui que jai indiqué (art. l.) pour la mefure de la Bafe d'Yarou$q u i, \ddot{j}$ ai conclu qu'il falloit appliquer les équations fuivantes à la mefure actuelle de la Bafe de Tarqui.

* Voyez art. XIII. 
$I^{\circ} \mathrm{O}^{\mathrm{t}, 062}$ à y ajoûter, pour réduire la mefure actuelle par échelons à la ligne horizontale au niveanu de Chinan, terme auftral de la $\mathrm{Bafe}$, ou à l'arc concentrique à la Terre, lequel on fuppofe paffer à la hauteur de ce terme.

$2^{\circ} \mathrm{O}^{\mathrm{t}}, \mathrm{O}$ I 3 à ôtér de cet arc, pour le réduire à fa corde.

$3^{\circ} \mathrm{O}^{\mathrm{t}}, \mathrm{I} 65$ à ajoûter à la longueur de la corde, pour convertir cette longueur en celle, de la ligne inclinée qui mefure la diftance d'un terme à l'autre de la Bafe.

$4^{\circ} 0^{i}, 30$ à fouftraire, pour réduire la corde de l'arc, paffant par Chinan, au niveau de Carabourou, plus bas que Chinan de 185 toifes. On aura donc

Mefure actuelle de la Bafe de Tarqui, par marches à différenś niveaux............... $5259^{\mathrm{t}, 200}$

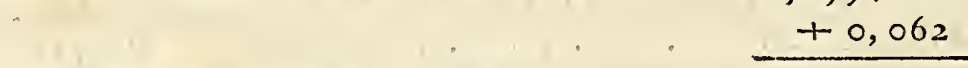

Réduite à l'arc au niveau de Chinan. . . . . 5259,262

A la corde du nême arc......... $\frac{-0,013}{5259,249}$

A la ligne droite d'un terme à l'autre. $\therefore \therefore \therefore \frac{+0,165}{5259,414}$

Corde de l'arc au niveau de Chinan ..... 5259,249

Réduite au niveau de Carabourou....... $\frac{-0,300}{5258,949}$

La Bafe de Tarqui, réduite au niveáu de Carabourou, comme on y a réduit tous les autres côtés de Triangles, fera donc de 5258 t,949, ou de près de 5259 toilfes.

Les deux Termes extrêmes de cette Bafe répondent au centre de deux meules de moulin que j’y ai fait placer; \& fur lefquelles j’ai gravé moi-même leur diftance mutuelle en toifes, fuivant la mefure actuelle, ainf que la direction de la Bafe par rapport aux régions du monde. J'ai eu foim de 


\section{DEGRÉS DU MERIDIEN.}

faire écorner ces deux pierres, pour qu'on ne fût pas tenté dé lés employer quelque jour à leur première déftination. J'ai pris la même précaution à l'égard de celles qui font placées au centre des fondemens de deux Pyramides que j’ai fait conftruire pour fervir de Termes à notre première Bafe à Yarouqui , \& dont jaurai occafion de parler ailleurs.

II refte à examiner fi la toife qui a fervi à mefurer la Bafe de Tarqui, eft précifément la même que celle qui a mefuré la Bafe d'Yarouqui, ou de combien elle en diffère.

\section{A R T I C L E XXI.}

Expériences fur les changemens de longueur d'une Toife de fer, expofée à différens degrés de chaleur.

$\mathrm{O}_{\mathrm{N}}$ fait que les métaux, en paffant du froid au chaud, ou d'une moindre chaleur à une plus grande, fe ditatent fenfiblement. Chacun de nous a cherché à reconnoître l'effet que cette caufe avoit pû produire fur les inftrumens qu'il a employés. II eft ici queftion de la Toife qui a fervi à toutes nos mefures; avant que de rendre compte de mes expériences, je crois devoir dire un mot de cette Toife.

Nous avions emporté avec nous en 1735 , une règle de fer poli, de dix-fépt lignes de Jargeur fur quatre lignes \& dèmie d'épaiffeur. M. Godin, aidé d'un Artifte habile, avoit mis toute fon attention à ajufter la longueur de cette règle fur celle de la Toife étalon, qui a été fixée en I 668 au pied de l'efcalier du grand Châtelet de Paris. Je prévis que cet ancien étalon, fait affez groffièrement, \& d'ailleurs expofé aux chocs, aux injures de l'air, à la rouille, au contact de toutes les mefures qui y: font 
76 MESURE DES TROIS PREMIERS

préfentées, \& à la malignité de tout mal-intentionné, ne leroit. guère propre à vérifier dans la fuite la Toife qui alloit fervir à Ia mefure de la Terre, \& devenir l'original auquel les autres. devoient être comparées. Il me parut donc très-néceffaire, en emportant une Toife bien vérifiée, d'en laiflè à Paris une autre de même matière \& de même forme, à laquelle on pût avoir recours s'il arrivoit quelqu'accident à la nôtre pendant su fi long voyage. Je me chargeai d'office du foin d'en faire faire une toute pareille. Cette feconde Toife fut conftruite par le même ouvrier, \& avec les mêmes précautions que la première. Les deux Toifes furent comparées enfemble dans une de nos affemblées, \& I'une des deux refta en dépôt à l'Académie : c'eft la même qui a été depuis portée en Lapponie par M. de Maupertuis, \& qui a été employée à toutes les opérations des Académiciens envoyés au Cercle Polaire. Celle que nous emportâmes, \& qui nous a toûjours fervi dans Je voyage, eft reftée à Quito, entre les mains de M. Godin, \& fera vrai-femblablement bien-tôt en France*; mais celle du Nord eft revenue, \& c'eft avec elle que j’ai fait, depuis mon retour , les expériences que je vais rapporter.

J'avois déjà fait à Quito plufieurs effais, tant fur notre Toife, que fur d'autres barres de fer, en les expofant alternativement au froid que produit le contact de la neige, \& à la plus grande chaleur du Soleil. J'en ai fait d'autres à Paris depuis mon retour, en fuivant le même procédé. Je fupprime ici le détail des uns \& des autres. Les variétés que j’ai rencontrées, \& celles qui fe trouvent dans les réfultats de

* On a nouvelle certaine du départ de $M^{\text {rs }}$ Godin \& de Jufleu le cadet, Lina pour Buenos aires par terre au mois d'Août 1748 . 
tous ceux qui ont fait les mêmes recherches, ont achevé de me convaincre qu'il y avoit peu de fuccès à efpérer, en enployant les méthodes ordinaires pour s'affurer d'une auff petite quantité que celle dont il eft ici queftion. En effet, quelle que foit l'attention de l'Obfervateur, \& même quelqu'adreffe qu'on lui fuppofe à tirer parti de la loupe, du compas, \&c。 Jes petites erreurs auxquelles, de l'aveu des plus habiles $\mathrm{Ar}$ tiftes, on eft encore expofé en opérant de cette forte, feront toûjours, dans le cas préfent, une partie confidérable de la quantité qu'on fe propoferoit de découvrir. J'ai donc cru que le moyen le plus fûr \& le plus décifif, pour déterminer avec précifion de combien s'alongeoit une barre de fer expofée à un certain degré de chaleur, étoit celui que j’avois déjà employé heureufement pour trouver la différence de longueur entre le Pendule à fecondes fous l'E'quateur, \& le Pendule à fecondes fous le Parallèle de Paris. Il n'étoit queftion, pour cela, que de faire de la Toife même un Pendule, \& de déduire, par le calcul, fon alongement, du moindre nombre de fes ofcillations dans un temps donné.

Pour cet effet, j’ai adapté à l'une des extrémités de la Toife de fer qui a fait le voyage du Nord, une fufpenfion à couteau femblable à celle de mon Pendule d'expérience que je décrirai ailleurs; \& encore plus parfaite. J'ai fufpendu de la même manière une autre Toife pareille dans une chambre voifine; en forte, néanmoins, qu'en ouvrant la porte de communication, je pouvois, d'un certain point, les voir ofciller toutes deux du même coup d'œil : j'ai rendu leurs vibrations ifochrones: j’ai enfuite échauffé avec un poële le lieu où j’avois renfermé la Toife du voyage du Nord : j’ai obfervé combiexs 
d'ofcillations la Toife expofée à l'air libre faifoit en un temps domné; \& fur ce fondement, j’ai déterminé la diftance de fon centre d'ofcillation à l'axe de fa fufpenfion, de $582^{\text {lig., }}, 56$.

J'ai remarqué enfuite combien cette Toife anticipoit, ou accéléroit dans fes vibrations, fur l'autre Toife, depuis que celle -ci ofcilloit dans un air où le Thermomètre de $M$. de Reaumur étoie monté de 13 degrés jufquà 5 s au déffus de la congélation: Par le nombre d'ofcillations dont la Toife échauffée tardoit fur la Toife expofée à l'air libre, j’ai conclu combien le centre d'ofcillation de la première avoit baiffé par la chaleü, \& combien la Toife totale sétoit alongée. Ce n’eft pas ici le lieu de rapporter tous les détails de trois expériencés, qui ont été répétées en trois jours différens, \& par divers degrés de chaleur. Il fuffit, quant à préfent, de dire, quaucune n’a duré moins de fix heures; \& qu'en prenaint un milieu entre les petites différences, \& ayant égard à toutes les circonftances, j’a trouvé que la chaleur, qui faifoit monter le Thermomètre de M. de Reauninur de dix parties, \& qui, par conféquent, dilatoit la liqueur de la centième partie du volume quelle occupe lors de la congélation de l'eau, faifoit alonger une Toife de fer, femblable à celle qui a fervi à nos opérations, de $\mathrm{o}^{\mathrm{lig}}$, , I I $\frac{1}{2} *$ : en forte que, fuppofant que les degrés d'extenfion, caufés par ta chaleur dans le fer, croiffent dans łe même rapport que ceux de la dilatation de l'efprit de vin (ce qui, dans les petites quantités, eft affez conforme à l'expérience) l'alongement de notre Toifé, qui répond à un degré du Thermomètre, fera de o tigs; o I I 5 , c'eft-à -dire; de plus de $\frac{1}{100}$, ou plus exactement, de $\frac{1}{87}$ de ligne.

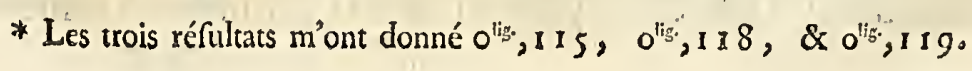


Ce réfultat s'éloigne beaucoup de celui des expériences que M. le Commandeur Don George Juan \& M. Godin ont faites à Quito fur la dilatation des métaux; mais il s'accorde aflez bien avec celles qu'ils firent dans le même temps fur leur condenfation ${ }^{2}$.

La conclufion qu'on tire de ces expériences dans cet ouvrage eft, que l'extenfion du fer, caufée par les augmentations de chaleur depuis le degré marqué i 3 fur le Thermomètre de $M$. de Reaumur, \& au deflus, eft plus que double de la contraction caufée par le froid, qui fait baiffer la liqueur dans ce même inftrument au deffous du même degré I 3 , jufqu'au terme de Ia glace, \& quelques degrés plus bas. On ne peut contefter des expériences plufreurs fois répétées, \& qui portent le caractère de la plus fcrupuleufe exactitude, telle qu'on étoit en droit de l'attendre, en pareil cas, d'obfervateurs auffi éclairés \& auffi exercés: mais je crois avoir un moyen de concilier des réfultats qui paroiflent $f_{1}$ peu conformes aux miens ${ }^{b}, \&$ de les faire convenir

a Obferv. aftrononic. y phyfic. Madrid 1748 , lib. IV, pag. 96 y 97.

${ }^{\mathrm{b}}$ Les Thernomètres 'de métal, appliqués à certaines horloges, ont une marche fenfiblement uniforme, \& analogue, au moins dans les petites variations, à celle des Thermomètres d'efprit de vin \& de mercure, tant en montant qu'en defcendant. L'expérience prouve donc, ainfı que le raifonnement, que lorfque le Thermomètre de liqueur monte de dix degrés, une barre de métal ne s'alonge que d'une quantité à peu près égale à celle dont elle s'accourcit lorfque le. Thermomètre baiffe de dix degrés. $\mathrm{Si}$ donc pour les expériences rapportées dans l'Ouvrage déjà cité, la Toife de fer s'eft plus alongée au Soleil à proportion, que le Thermomètre n’a monté, il faut que la Toife ait été expofée à un plus grand degré de chaleur que, le Thermomètre; \& c'eft auffi ce qui a dû arriver, lorfqu'on a couché horizontalement la Toife de fer fur un appui de pierre, de brique, ou même fur la terre déjà échauffée par les rayons du Soleil, reçûs prefque perpendiculairement à Quito. Il eft clair que la Toife 
avec mes expériences, fans fuppofer: gratuitement que la ditatation des métaux \& leur condenfation fuivent des loix totalement différentes; ce qui préfente au moins l’apparence d'une contradiction.

\section{A R T I C L E XXII. \\ Comparaifon de la longueur de la Toife lors de la mefure des deux Bafes.}

Pendant notre féjour dans la Province de Quito, nous avons remarqué que la chaleur augmentoit ou diminuoit, généralement parlant, dans la raifon du plus ou du moins d'élévation du fol au deffus du niveau de la Mer.

Le niveau d'Oyambaro, terme auftral de notre première Bafe, près de Quito, eft inférieur au niveau de Ouaoua-tarqui, terme feptentrional de notre feconde Bafe, près de Cuenca, d'environ treize toifes*: mais comme le terrein de la première Bafe a beaucoup plus de pente que celui de la feconde,

prenoit alors le plus grand degré de chaleur qui lui pût être communiqué par les rayons directs, \& par les objets voifins qui en étoient pénétrés; au lieu que le Thermomètre, \& le mur vertical auquel il étoit attaché, recevoient d'autant plus obliquement les rayons du Soleil, que ceux-ci tomboient plus à plomb : le Thermomètre a donc dû moins nonter à proportion, que la Règle de fer n’a dû s'étendre. Il n'en étoit pas de même dans l'expérience inverfe, lorfque le Thermomètre \& la Toife paffoient de l'air tempéré d'une chambre, dans la neige, dont on les couvroit tous deux également, \& dont ils recevoient le même degré de froid : les degrés de condenfation du métal \& de la liqueur devoient alors être à peu près proportionnels; auffi le réfultat de cette expérience approche - $t-i l$ beaucoup de celui des niennes.

* Voyez pages 55 \& 56 . 
le milieu de celle-ci eft plus élevé d'environ cent toifes que le milieu de la première. Il eft vrai qu'une fi petite différence de hauteur pourroit à peine produire un effet fenfible, fi quelques autres circonftances locales ne s'y joignoient. Quoi qu'il en foit, la température de l'air dans la plaine d'Yarouqui, où nous mefurâmes notre première Bafe, eft, pour l'ordinaire, plus chaude que dans la plaine de Tarqui, où nous mefurâmes in feconde.

Cela pofé, il femble que la Toife de fer, à laquelle nous rapportions nos mefures, auroit dû fe contracter, dı moins um peu, à Tarqui ; \& par conféquent, que nous aurions dû compter un plus grand nombre de toifes, en mefurant cette plaine, que fí notre Toife de fer eût confervé la même extenfion qu’à Yaronqui. Je fuis néanmoins porté à croire qu'elle s'eft pluftôt alongée que racourcie dans le temps de la mefure de notre feconde Bafe à Tarqui; \& voici les raifons fur lefquelles je me fonde.

Quand je dis que la plaine d'Yarouqui eft fenfiblement plus chaude que celle de Tarqui; cela doit s'entendre de la partie inférieure de la plaine d'Yarouqui, du côté où elle s'approche de Carabourou, terrein aride \& fablonneux, \& le plus chaud de tout le canton.

C'eft en partant de ce lieu, où étoit fixé le terme feptentrional de la Bafe, que nous commençâmes, M. Bouguer \& moi, à en mefurer la longueur, en montant vers Oyambaro, d'où M. Godin avoit commencé fa mefure en defcendant vers Carabourou. Il fe paffa plufienrs jours fans que nous pûfflons, M. Bouguer \& moi, comparer les perches de bois que nous pofrons fur le terrein, à la Toife de fer qui avoit fervi à 
82 MESURE DES TROIS PREMIERS

les étalonner. Cette opération s'étoit faite précédemment ì Oyambaro, terme aufral de la Bafe, plus élevé de 126 toifes que Carabourou, terme boréal, \& fitué au pied d'une montagne, dans une tempériture d'air affez froide; jufques- łà que nous y avóns vû plufieurs fois le matin, le Thermomètre ne marquer que trois ou quatre degrés au deflus du terme de la glace; c'eft-à-dire, près de vingt degrés moins qu'il ne marquoit ordinairement l'après-midi à Carabourou Nous étions à peu près au tiers de notre mefure en montant, lorfqu'il nous arriva d'Oyambaro une Règle de fer de cinq pieds, laquelle, ainfi que nos perches, y avoit été ajuftée fur la Toife apportée de France. Nous comparâmes les jours fuivans les perches à cette Règle : cela fe faifoit le matin avant que de nous mettre au travail, \& dans le temps le plus froid de la journće. Comme nous approchions chaque jour d'Oyanbaro, bien - tôt après nous allâmes nous y ctablir; \& ce fut en ce lieu mème que fe firent déformais les comparaifons à la Toire originale. Nous effuyâmes alors des vents, de Ia pluie, \& un temps afiez froid, tandis que la difficulté dus terrein, qui, vers cette extrémité de la Bafe, nous obligeoit à prendre des à-plombs prefque à chaque pas, retardoit encore notre marche. On peut donc ftatuer que la Toife, à laquelle ont été rapportées nos mefures prifes fur le terrrein, lors de nos opérations de la Bafe d'Yarouqui, eft, à très-peu près, la Toife du climat d'Oyambaro, c'eft-à-dire, celle d'un lieu où la hauteur moyenne du Thermomè̀re différoit très-peu, \& peut - être par défaut, de celle des caves de l'Obfervatoire de Paris, laquelle répond dans le Thermomètre de $M$. de Reaumur, à 1 od $\frac{1}{2}$ au deffius de la congélation. 
A Tarqui, nous n'avions point de Thermonètre; mais le temps fut très-beau pendant toute la durée de la mefure de cette feconde Bafe: nous opérâmes dans la faifon la plus chaude de l'année, \& nous étions fouvent en fueur. Nous faifions porter avec nous une Toife étalonnée de fer brut, à laquelle nous comparions tous les jours nos perches fur le terrein même, à l’ombre à la vérité; mais en plein jour, \& quelquefois à l'heure de la plus grande chaleur. Enfin, le jour que nous paffâmes le marais, dont j’ai parté plus haut, nous fmîmes notre journée par mefurer plus d'un quart de lieue les pieds dans l'enu, ou dans un terrein où nos perches, imbibées d'eau, dûrent, comme l'expérience le fait voir, s'alonger confidérablement; \& la nuit nous ayant furpris dans cette occupation, ce ne fut que le lendemain que nosıs pûmes les comparer à Ia Mefure de fer: or il eft vifible qu'elles devoient alors avoir perdu, en fe féchant, une partie de leur extenfín.

Non feulement les faits que je viens d'alléguer me font très-préfens; je les trouve même détaillés fur mon Journa! elobfervations. La circonftance feule d'avoir mefuré l'efpace de 600 toifes, dans l'eau jufqu’à mi-jambes, fans reffentir ni incommodité, ni la plus légère impreffion de froid, fuffit pour faire juger, par comparaifon à d'autres expériences, que le Thermomètre devoit être alors à 20 degrés, \& peutêtre plus, au deflus du terme de la glace: d'où je conclus, que la hauteur moyenne du Thermomètre, pendant la mefure de notre Bafe à Tarqui, étoit au moins de i 6 à I 7 degrıés, c'eft-ì-dire, plus grande de 6 à 7 degrés qu'à Oyambrtro; que par conféquent la Toile de fer, \& les perches de bois dont cette Toife a fervi à fixer la longueur dans. la plaine de 


\section{MESURE DES TROIS PREMIERS}

Tarqui, étoient plus longues que celles qui nous avoient fervi dans Ia plaine d'Yarouqui; \& qu'enfin cet alongement, en nous faifant compter moins de mefures, a dû nous faire trouver la Bafe de Tarqui plus courte, que fi la Toife n'eût fouffert aucune variatioñ depuis la mefure de la première Bafe.

L'équation que cette obfervation me fournit, tendroit à rapprocher la valeur conclue, de celle qui a réfulté de ma mefure actuelle de cette même Bafe; mais je me contenterai de n'appliquer aucune équation, ni en plus, ni en moins; pour la diverfité de la température des deux terreins. En effet, quoi quili en foit de la difcuffion précédente, \& fans entrer dans un détail, peut-être trop fcrupuleux, il eft certain, \& on ne peut nier, I ${ }^{\circ}$ Que nous n’ayons éprouvé, en mefurant la Bafe d'Yarouqui, un moindre degré de chateur que l'ordinaire, vû la faifon pluvieufe alors, \& notre plus long féjour dans le voifinage d'Oyambaro. $2^{\circ}$ Que le temps fec \& chaud qu'il fit lorfque nous mefurâmes à Tarqui la feconde Bafe, n'ait adouci le petit froid qu'on y reffent ordinairement : d'où il s'enfuit généralement, que ces deux températures, déjà affez peu différentes par elles-mêmes, doivent au moins s'être approchées, fi elles n'ont pas même empiété, pour ainfi dire; l'une fur l'autre; \& quainfı on peut, fans inconvénient, les fuppofer égales. Enfin, foit qu'on prenne un terme moyen entre les plus grands degrés de chaud \& de froid que nous avons éprouvés dans les plaines d'Yarouqui \& de Tarqui, \& qui font à peu près $2 \& 26$ degrés au deffus du terme de Ia glace dans le Theimomètre de M. de Reaumur; foit qu'on s'en tienne au milieu entre I $0 \frac{1}{2} \& 16 \frac{1}{2}$ degrés, qui marquent les deux températures moyennes que je viens d'affigner aux 
terreins des deux Bafes, pour le temps où nous les avons mefurées, on aura à très-peu près le degré I 3 au deffus de o; \& c'eft précifément celui que le Thermomètre de $M$. de Reaumur marquoit à Paris en I 735, lorfque notre Toife de fer fut étalonnée fur celle du Châtelet par M. Godin.

Ce degré de chaleur peut être pris pour celui de la température moyenne de l'air en France, \& me difpenfe par conféquent de toute réduction fur la longueur de notre mefure, pour la variation de la Toife par la différence des climats de Paris \& de Quito.

\section{A R T I C L E XXIII.}

Comparaifon de la mefure actuelle de la Bafe de Tarqui à fa longueur calculée.

LA mefure actuelle de la Bafe de Tarqui, réduite au niveau de Carabourou, a été trouvée de $525.8 \mathrm{t}, 949$, c'eft-à-dire, de près de 52.59 toifes; \& par une fuite de Triangles, dont le premier côté eft la Bafe d'Yarouqui, fa valeur a été conclue de $5260^{t}, 03$. Cette différence d'une toife \& quelques pouces, entre la mefure actuelle \& le réfultat du calcul, deviendroit moindre par l'équation qui convient à la diverfe température des terreins des deux Bafes, conformément aux remarques de l'Article précédent: mais comme ces remarques, toutes fondées qu'elles font, n’ont été faites qu'après coup, \& qu'on pourroit imaginer que je n'en ferois ufage que parce qu'elles favoriferoient l'accord de notre mefure actuelle de la Bafe de Tarqui avec la valeur de cette même Bafe, conclue par le calcul de mes Triangles, je m'abftiendrai d'employer l'équation qu'elles. pourroient me fournir.

$\mathrm{N}$ iij 
J'aurois auffi pû réduire la différence, d'une toife à̀ une demi-toife, en employant le calcul d'une autre fuite d'angles, dans lequel j’ai fubftitué à quelques-uns de nos Tṛiangles Planche II, principaux les Triangles auxiliaires, repréfentés par des lignes fig. I. ponctuées. Mais ayant fait réflexion, que nous avions trois mefures différentes des mêmes Triangles, indépendantes l'une de l'autre, exécutées avec différens inftrumens, \& par divers Obfervateurs; enfin n'ayant pas jugé que mon fyftème de Triangles auxiliaires, où il n'y en avoit que fept de nouveaux, fût affez différent du premier pour mériter le nom de nouvelle Suite de Triangles, j’ai fupprimé dans cet Ouvrage le calcul que $j$ 'en avois fait, \& jabandomne encore l'avantage que j'en pourrois tirer, pour rapprocher mon réfultat de la mefure actuelle de la Bafe de Tarqui. A quoi bon, en effet, chercher à concilier cette différence; puifque fi nous avons été fouvent d'accord dans la toife, \& quelquefois dans le pied; fur une longueur de is à 20 mille toifes, conclue par des obfervations faites avec différens Quarts - de-cercle; il faut avouer quil nous eft auffi quelquefois arrivé, malgré toutes nos précautions, de trouver par les différentes mefures d'une même Suite de Triangles, des différences de deux, ou de près de trois toifes fur une pareille longueur, comme il s'en trouve, diuns le cas préfent, une d'une toife fur 5260 toifes, entre Ia mefure actuelle $\&$ la mefure conclue?

Je ne ferai donc point de vains efforts pour diffmuler une légère erreur, de laquelle il n'eft pas poffible de répondre fur un aufí grand nombre d'opérations combinées. Quand le caicul s'accorderoit parfaitement avec la melure actuelle de la feconde Bafe, tout ce qu'on feroit en droit d'en conclurre; 
c'eft que les erreurs fe feroient probablement compenfées. Après une fuite de 32 Triangles, qui mefurent une diftance de quatre-vingts lieues, une toife feule de différence fur le dernier côté eft peut-être plus propre à fervir de preuve de l'exacitude des opérations, que de raifon pour donter de leur jufteffè.

Avant que de paffer outre, il ne fera pas hors de propos d'examiner quel changement cette différence d'une toife fur la Bafe de Tarqui doit apporter à la longueur totale de la Mónridienne.

\section{A R T I C L E XXIV.}

$S_{i}$ toute erreur d'obfervation, qui fera trouver trop. long le dernier cốté conchu des Triangles de la Méridienne, doit aulfi néceffairement faire trouver trop longue la Méridienne calculíe.

C

ET T e queftion ne pent être décidée que par la confio dération des élémens qui entrent dans la détermination des deux quantités dont on fe propofe d'examiner la relation ; quantités, qui font dans le cas préfent, la Bafe de Tarqui, \& Ia Iongueur conclue de la Méridienne.

Quant à la recherche de la longueur de la Bafe de Tarqui, ainfı que de celle de chaque côté de Triangle de la Méridienne, on y a toûjours procédé par une analogie de cette efpèce. Le finus de l'angle oppofé au côté précédemment connu, eft à ce côté; comme le finus de l'angle oppofé au côté cherché, eft à ce dernier côté. Il s'enfuit déjà de là, que chaque côté peut être exprimé par une fraction qui ait pour numérateur le produit de la première Bafe mefurée à Yarouqui, par les funus de tous, 
les angles oppofés aux côtés fuccefivement conclus; \& pour dénominateur, les produits des finus de tous les angles oppofés aux côtés qui ont fervi à conclurre. On en peut encore tirer cette conféquence, que la longueur de la Bafe de Tarqui dépend de la grandeur des finus des angles oppofés à tous les côtés qu'on a conclus dans le calcul des Triangles précédens, \& de la petiteffe des finus des angles oppofés à ceux d'où on a conclu.

Quant à la longueur de la Méridienne, elle a été déduite de l'addition de fes différentes portions; \& chacune de ces portions l'a été d'une analogie différente de la précédente, \& où l'on a confidéré cette portion comme côté d'un Triangle rectangle fictice, dont le côté conclu du Triangle obfervé étoit l'hypothénufe.

Tout ceci, tant ce qui regarde les côtés conclus des Triangles obfervés, que ce qui a rapport au calcul des portions correfpondantes de la Méridienne, deviendra plus clair par un exemple.

Planche I, Je choifis le Triangle XVII de la Méridienne. Je fuppofe fig. 6 .

d'abord que dans ce Triangle repréfenté par $D I Z$, le côté $D I$ ait été conclu par la réfolution des Triangles précédens: je veux en déduire,

$I^{\circ} \mathrm{La}$ longueur du côté $I Z, 2^{\circ}$ Lạ longueur de la portion correfpondante $\zeta \zeta$ de la Méridienne.

Pour trouver la valeur de $I Z$, je fais cette analogie fin. $Z: D I$ $::$ fin. $D: I Z$; d'où je tire $I Z=\frac{D I \times \text { fin. } D}{\sin Z \text {. }}$. Or au lieu de $D I$ qui fe trouve dans cette expreffion, on peut fubftituer $f_{a}$ valeur tirée d'une femblable analogie, qui, dans le Triangle précédent, a fervi à conclurre $D$ 1: \& ainf 


\section{DEGRÉS DU MERIDIEN.}

ainf fucceffivement en remontant de Triangle en Triangle jufqu'à $C O$, première Bafe mefurće à Yarolqui (Triang. 1). Subftituant donc de cette forte toutes les valeurs trouvées des côtés précédemment conclus, \& nommant $a, b, c, d, e, \delta c$. les finus des angles oppofés aux côtés conclus, $\alpha, \beta, \gamma, \delta, \varepsilon, \&$ c. fes finus des angles oppofés aux côtés qui ont fervi à conclurre, on aura à la fin $I Z=\frac{c O \times a \times b \times c \times d \times e^{i} \& c .}{\alpha \times \beta \times 2 \times d \times \varepsilon}$.

De même, fr au lieu de la valeur du côté $I Z$ on cherchoit celle du dernier côté des Triangles de la Méridienne, c'eft-à-dire, de la Bafe de Tarqui (Triang. XXXII), on auroit

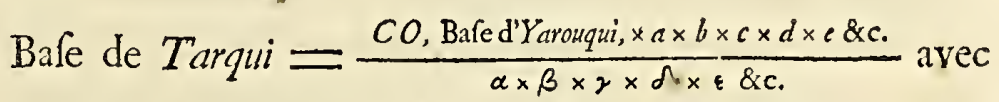
33 termes au numérateur, \& un de moins au dénominateur.

Quant à la portion correfpondante $\iota \zeta$ de la Méridienne. c'eft-à-dire, à la portion comprife entre les perpendiculaires $I_{l}, Z \zeta$, tirées des points $I \& Z$ fur la Méridienne, on la trouvera en tirant par le point $I$ une parallèle $I Z$ à la Méridienne, \& en formant le Triangle rectangle $I Z_{Z}$, dont le côté $I Z$ connu par l'analogie précédente, fera l'hypothénufe; ce qui donnera cette autre analogie, fill, total : $Z I::$ fin. $I Z_{Z}$ $: I_{Z}=1 \zeta$, d'où l'on tirera $\iota \zeta=\frac{Z I \times \text { fini' } I Z_{\tau}}{\text { fir. total }_{\text {. }}}$.

II s'enfuit de là, que la longueur conclue d'une portion quelconque $\zeta \zeta$ de Ia Méridiemne, laquelle corrếpond à un côté de Triangle obfervé, dépend tout à $\mathrm{l}$ fois, de la grandeur de ce côté, \& de la grandeur du finus de l'angle $I Z Z$. complément de l'angle que fait ce même côté avec la Méri: diemne. 
Il entre donc dans la détermination de la longueur des portions de la Méridienne, telles que $\zeta_{9}$, un nouvel élément qui n'a contribué en rien à la détermination des côtés des Triangles obfervés; \& c'eft l'angle $Z$ I Z que le côté $I Z$ fait avec Ia Méridienne. S’il étoit poffible de fuppofer cet angle exempí d'erreur, fous prétexte que nous avons fouvent vérifié, par des obfervations d'azimuth, la direction du côté $I Z$, par rapport aux régions du monde, dès-lors l'autre angle aigu du Triangle rectangle, feint fur ce même côté, feroit aufli exempt d'erreur; le côté $I Z$ de ce même Triangle rectangle, ou la portion correfpondante $\iota_{\zeta}$ de la Méridienme, laquelle lui eft égale, ne pourroit plus varier que proportionnellement à $I Z$; \& par conféquent l'alongement de chaque portion de la Méridiemne ne dépendroit plus que des mêmes caufes d'où s'enfuit l'alongement du côté correfpondant des Triangles obfervés. Mais cette fuppofition renfermeroit ine forte de contradiction, en ce que toute erreur dans les angles des. Triangles obfervés, doit, à moins d'une compenfation nullement vrai-femblable, en entrainer une dans l'angle que les côtés de ces Triangles font avec la Méridienne; puifqu'on ne découvre la valeur de ce dernier angle, par exemple, de l'angle $\mathrm{Z}$ I $Z$, qu'en faifant à l'angle de la première Bafe avec la Méridienne, des additions \& des fouftractions fucceffives d'autres angles obfervés.

La grandeur de chaque côté de Triangle, \& celle dé chaque portion de Méridienne, correfpondante à un côté, dépendent donc de deux fortes d'élémens; les uns particuliers à l'une de ces grandeur's exclufivement; les autres communs à toutes les deux, mais qui fe combinent de manières 
très-différentes dans la détermination de ces mêmes grandeurs. A moins donc que de parcourir toutes les fuppofitions poffbles, tant fur le nombre \& la difpofition des Triangles, que fur la diverfe combinaifon des erreurs d'obfervation dans les angles, \& d'en examiner tous les réfultats; on ne fauroit être en état de conclurre en général; que l'excès de longueur de la dernière Bafe calculée, doive entran̂ner un excès dans le calcul de la longueur de la Méridienne.

Mais , fr, reftreignant la queftion à notre Méridienne précifément, on a égard aux circonftances particulières de notre mefure, telles que la difpofition de nos Triangles, dont Ia fuite s'écarte peu de la direction de la Méridienne, leur forme oxigone, aflez approchante, pour lordinaire, de l'équilatérale \&c; ces reftrictions feront qu'on aura beaucoup moins de cas à examiner, pour fe décider fur la queftion propofée. Enfin l'accord de nos différentes Suites de Triangles, \& des différentes mefures d'une même Suite par divers obfervateurs \& avec divers inftumens, donnera aux erreurs d'obfervation des limites étroites, qui augmenteront beaucoup la probabilité des conclufions qu'on aura tirées.

Après d'affez longues recherches, auxquelles j’ai appliqué Ia théorie de M. Cotes *, je me fuis convaincu, que dans le plus grand nombre de cas, pris abftraitement, \& fur-tout dans le plus grand nombre de ceux qui font applicables ̀̀ nos Triangles, l'alongement qu'une erreur dans l'obfervation de quelque angle, produit dans un côté conclu par le calcuĺ, emporte néceffairement l'alongement de la portion correfpondante de la Méridienne calculée; d'où il s'enfuit que dans'

* De ajfimatione errorum in mixtâ mathefi.

\section{$O$ ij}


Ia totalité de ces mêmes cas, il entraine cet alongement avec une très-grande probabilité.

La forme que je me fuis prefcrite dans cet ouvrage ne me permet pas d'entrer ici dans le long détail d'une difcuffion géométrique qui n'a rien d'attrayant; \& je crois que le lecteur me faura d'autant plus de gré de la lui épargner, que lorfqu'on veut, dans le cas préfent, rendre générales les propofitions démontrées, \& éviter les équivoques, l'énoncé des théorèmes devient fouvent prefqu'auffi long \& quelquefois auffi difficile à entendre que la démonftration même.

Je me bornerai donc à la confidération fuivante. II n'eft pas à préfumer, que l'excès de longueur d'une toife, trouvé par le calcul fur la mefure actuelle de la Bafe de Tarqui, provienne des feules erreurs commifes dans l'obfervation des angles du dernier Triangle, ou même dans l'obfervation des angles des feuls Triangles qui précèdent immédiatement le dernier. It faudroit pour cela fuppofer dans ces angles des erreurs trop confidérables, fur des angles ou, par les précautions dont on a rendu compte, ce feroit beaucoup, pour l'ordinaire, que de fuppofer r o fecondes d'erreur. II y a donc toute apparence que la plufpart des côtés calculés des Triangles antérieurs, qui ont fervi à conclurie la Bafe de Tarqui, ont péché plus ou moins en excès, c'eft-à-dire, ont été conclus trop grands. Mais nous venons de voir que l'alongement d'un côté de Triangles a dû produire le plus fouvent l'alongement de la portion correfpondante de la Méridienne : Ia plufpart des portions de la Méridienne doivent donc très-vrai-femblablement avoir été conclues trop longues, \& à bien plus forte raifon cela doit-il être de da 


\section{DEGRÉS DU MERIDIEN。}

Méridienne totale qui en eft la fomme.

Je conclus, en répondant directement à la queftion que je me fuis propofée; que toute erreur d'obfervation qui feroit trouver par le calcul le dernier côté d'une fuite de Triangles plus long qu'il ne l'eft réellement, ne fera pas néceffairement, \& dans tous les cas, attribuer trop de longueur à une ligne qui traverferoit cette fuite de Triangles, comme la Méridienne fait ici ; mais que dans le cas dont il eft queftion, il y a un trèshaut degré de probabilité, ce qui fait prefque une certitude morale, que la même caufe d'erreur qui a fait trouver la Bafe de Tarqui plus longue par le calcul que par la mefure actuelle, nous a fait auffi conclurre notre Méridienne plus longue qu'elle' ne l'étoit en effet. Il ne rête plus qu'à évaluer cet excès.

\section{A R T I C L E XXV.}

De combien une différence d'une toife fur la Bafe de Tarqui doit changer la longueur de la Méridienne. LL y a un nombre infini de fuppofitions d'erreurs poffibles qui feroient toutes trouver par le calcul la Bale de Tarqui trop longue d'une toife.

Comme il n'y a point de raifon abfolument décifive pous préférer l'une de ces fuppofitions à l'autre, il eft à propos de prendre un milieu entr'elles: \& ce milieu fe peut chercher par différentes voies.

On pourroit fuppofer, mais gratuitement \& fans aucune vrai-femblance, que tous les angles qui précèdent le Triangle fur la Bafe de Tarqui, font exempts d'erreur, \& que l'erreux" d'une toife, ou de $\frac{1}{5000}$, qu'on a trouvé de trop dans Ie 
calcul de cette $\mathrm{Ba}$ e, n'a été commife que dans les angies de ce derniel Triangle. Il eft clair, qu'en ce cas, Ia Méridienne calculée ne feroit alongée par cette eireur que dans fa portion correfpondante à la Bafe de Tarqui, \& d'une quantité plus ou moins grande, fuivant l'angle de cette Bafe avec la Méridienne; de forte que lalongement total de la Méridienne, ou l'excès de fa longueur calculée fur fa longueur vẹritable feroit d'une toife, fi la Bafe de Tarqui étoit dirigée du Nord au Sud, ou parallèlement à la Méridiemne; qu'il fe réduiroit à o, fi la direction de la Bafe coupoit la Méridienne à angles droits, \& qu'il eft d'environ cinq pieds, parce que cette Bafe décline en effet de la Méridienne de 32 degrés 25 minutes.

On pourroit également fuppofer, que toute l'erreur procède des angles du premier Triangle fur la première Bafe; que le premier côté conclu a été trouvé trop long de $\frac{1}{5000}$ \& que tous les angles fuivans ont été bien obfervés. Dans cette fuppofition, tous les côtés auroient été àlongés, ainf que le premier côté \& dans le même rapport : \& le dernier qui eft la Bafe de Tarqui, ayant environ 5000 toifes, le calcul lui eût donné une toife de trop; par conféquent 35 à 36 toifes de trop à la Méridienne, c'eft-à-dire à peu près $\frac{1}{5000}$ de fa longueur totale, Iaquelle diffère peu de 180000 toiles.

Ces deux fuppofitions s'éloignent également de la vrai-femblance, \& par des chemins directement oppolés. On peut donc les regarder comme deux cas extrêmes, \& comme des efpèces de limites. Si on prend le milieu des deux rélutats. entre une toife \& 36 , on aura environ i 8 toifes pour l'excès de la Méridienne calculée fur la véritable.

Effayons une autre fuppofition. Que l'erreur ait commencé 


\section{DEGRÉS DU MERIDIEN.}

dès les premiers Triangles: qu'elle ait été d'abord très-petite: qu'elle fe foit enfuite accrue infenfiblement toûjours dans le même fens, jufqu'à produire un peu plus d'une toife fur le dernier côté, long de 5260 toifes, \& qui eft ici la Bafe de Tapqui ; la quantité dont le calcul feroit trouver en ce cas la Méridienne totale trop longue, feroit à peu près égale à la fomme d'une progreffion arithmétique croiffante, de 36 termes; dont le premier feroit prefque $=0$, \& dont le dernier feroit de près d'une toife. Or fi l'on fait la fomme des termes de cette progreffion, on la trouvera égale à près de I 8 toifes; c'eft-àdire, au nombre déjà trouvé en prenant un milieu entre les deux fuppofitions précédentes; \& en effet, la dernière de ces fuppofitions ne pouvoit manquer de donner la moitié du réfultat de celle qui a fait trouver 36 toifes pour l'alongement total de 1 a Méridienne; le cas de la première pouvant être reprélenté par un parallélogranıme, qui, fur une longueur donnée, auroit pour hauteur une toife, \& celui de la feconde, par un Triangle de même Bafe \& de même hauteur, \& par conféquent moitié du parallélogramme.

\section{A.R T I C L E XXVI.}

Autres manières de trouver l'équation de la longueur de la Méridienne pour une toife de différence fur la longueur de la Bafe.

D an s la vûe de faire de notre Bafe de Tarqui l'ufage naturel auquel elle étoit deftinée; \& pour donner à cette Bafe, qui avoit été mefurée avec le même foin que celle d'Yarouqui, \& fus un terrein plus favorable, autant de part quià la pre: 


\section{MESURE DES TROIS PREMIERS}

mière dans la détermination de la longueur du degré; voicí le plan que javois fuivi, en faifant mes premiers calculs de la Méridienne, en 1739 \& 1740 . Je pris pour fondement de ce calcul la Bafe mefurée à Yarouqui, $(T r .1), \&$ je réfolus tous Planche II, les Triangles fuivans vers le Sud, jufquau Triangle XIV , \& au fig. I.

côté terminé par les Signaux de Moulmoul \& d'Ygoalata, lequel eft fitué affez exactement au milieu de la longueur de notre arc de trois degrés. Je commençai enfuite un nouveau calcul, en partant de la Bafe mefurée de Tarqui à l'extrémité fud de Ia Méridienne; \& prenant la mefure actuelle de cette Bafe pour premier côté, je réfolus, en remontant vers le Nord, tous les Triangles jufqu'au mêne côté de Moulmoul à Ygoalata, déjà conclu par la Bafe d'Yarouqui. Je trouvai alors ce côté plus court d'environ une toife que par le premier calcul; ce qui provenoit de la même caufe qui me fait trouver aujourd'hui la Bafe de Tarqui plus longue que la mefure actuelle. Enfin je pris pour Ia vraie longueur de ce côté, la longueur moyenne entre les deux qui avoient été conclues par les deux différentes Bafes, ne voyant pas plus de raifon de déférer à l'une qu’à l'autre.

Ce procédé me donne un moyen de corriger les côtés intermédiaires des Triangles des deux moitiés de la Méridienne. Je prends pour exemple fa moitié feptentrionale depuis Moulmoul jufques à Cotchefqui. Je regarde la diftance corrigée de Moulmoul à Yoagolata comme une troifième Bafe mefurée réellement: je calcule fur la longueur de cette nouvelle Bafe, les Triangles qui s'y appuient fucceffivement, en remontant vers le Nord, jufquà celui qui fe trouve à moyenne diftance entre Moulmoul \& Cotchcfqui, au quart de Ja Méridienne du côté du Nord, par exemple, jufquiau côté auftral du Triangle VIII, entre 
les Signaux de Milin \& de Papa-ourcou: je compare la longueur de ce côté, troúvée par ce nouveau calcul, à celle qui avoit été trouvée en premier lieu en partant de la Bafe d'Yarouqui, \& qui étoit un peu moindre: je prends le milieu des deux longueurs conclues pour la vraie; \& je regarde cette diftance, ainf corrigée, comme une quatrième Bafe. Celle-ci me fert de même à corriger un autre côté à moyenne diftance entre celui-ci \& la Bafe d'Yarouqui; \& ainfi de fuite, je corrige fucceffivement tous les côtés des Triangles, \& à proportion les parties correfpondantes de la Méridienne; je prends la fomme de celle-ci, pour avoir la longueur totale coírigée, \& je trouve encore la Méridienne plus courte de I 8 toifes que par le premier calcul. Ce procédé, quoique très-différent en apparence des précédens, me donne précifément le même réfultat; \& il doit le domner, puifqu'il renferme tacitement la fuppofition précédente d'une erreur accrue uniformément \& du même fens.

Enfin une autre manière fort fimple de confidérer ta chofe, \& de faire pareillement fervir ta mefure àctuelle de ta Bafe de Tarqui à la correction de la longueur de la Méridienne, trouvée par le premier calcul fait fur Ia Bafe d'Yarouqui, ce feroit de faire un calcul rétrograde, dont la Bafe de Tarqui calculée feroit le fondement; puis de déduire de ce nouveau calcul la longueur de la Méridienne, de la même manière qu'on l'avoit d'abord déduite de la mefure actuelle de la Bafe d'Yarouqui; \& de prendre enfin pour longueur vraie de la Méridienne la longueur-moyenne entre les deux qu'on auroit conclues par ces deux différentes voies. On voit que par ce procédé, les deux mefures des deux Bafes auroient également part 
à la détermination de la longueur de la Méridienne $\&$ à celle de la longueur du degré; mais il n'eft pas même néceffaire pour cela de faire effectivement ce nouveau calcul; on peut s'en épargner l'embarras, \& cependant en trouver le réfultat par les confidérations fuivantes.

Le ealcul fait fur la mefure actuelle de la première Bafe; qui eft celle d'Yarouqui, a fait conclurre la longueur de la feconde Bafe, c'eft-à-dire, de la Bafe de Tarqui, d'une toife de plus fur 50oo, qu'on ne l'a trouvée par la mefure actuelle; je néglige ici les fractions : \& par le même calcul on a conclu Ia longueur totale de la Mćridienne, comme on a vî art. XVIII, de près de 177808 toifes. Si donc on refaifoit le calcul, en rétrogradant de la feconde Bafe conclue à la première, il eft évident qu'on retrouveroit la vraie valeur de la première Bafe, \& la même longueur de la Mériclienne: \& fi dans cettè nouvelle fupputation on prenoit pour fondement, non la feconde $B a f e$ conclue, mais $f_{a}$ vraie longueur, telle que la donne la mefure actuetle, c'eft-à-dire, une longueur moindre d'une toife fur 5000, que celle qu'on lui avoit attribuée; il n’eft pas moins clair qu'on trouveroit tous les côtés de Triangles plus courts proportionnellement, ou de $\frac{1}{5000}$ plus courts que par le calcul précédent. Il en feroit de même de toutes les parties correfpondantes de la Méridienne, \& par conféquent de la longueur totale de cette Ligne. Et comme fa cinq-millième partie de 177808 toifes eft environ 36 toifes, la longueur totale de la Méridienne feroit donc par ce nouveau calcul de 177808 toifes - 36 .

Si l'on prend enfuite le milieu des deux longueurs, l'une de 177808 toifes, conclue par le calcul fait fur la mefure 
actuelle de la première Bafe, l'autre de $177808^{t}-36$, qu'on trouveroit en refaifant le calcul fur la mefure actuelle de la feconde Bare, on aura pour mefuremoyenne 1778 o 8 - I 8; c'eft-à-dire, r 8 toifes de moins que ce qu'on avoit trouvé par le premier calcul.

Nous revenons donc toûjour's à une même conclufion : \& en effet les fuppofitions précédentes, \& ce nouveau procédé, ne diffèrent point dans le fond; \& ce ne font qu'autant de divers afpects du même objet. Cette manière de le confidérer eft peut-être la plus fimple de toutes; mais elle ne s’eft préfentée à moi que la dernière.

J'ai fuppofé une toile entière d'erreur fur la Bafe de Targui, conclue par la fuite du calcul de mes Triangles, depuis la Bafe d'Yarouqui, quoique j’euffe, comme je l'ąi remarqué, différens moyens de réduire cette quantité à une beaucoup moindre. J'ai regardé cette différence d'une toife comme le produit d'une fuite d'erreurs toûjours croiffantes, \& dans le même fens; fuppofition qui pafle les bornes de la vrai-femblance. Cependant tout ce qui s'enfuivroit de là, c'eft qu'il y auroit près de I 8 toifes, oụ $\frac{1}{10000}$, à retrancher de la lorgueur totale de notre arc du Méridien de trois degrés fept minutes, c'eft-à-dire, moins de fix toifes par degré : or qu'eft-ce que fix toifes, quand on confidère que chaque feconde d'erreur fur l'amplitude de l'arc, quantité plus petite que celle dont aucune induftrie humaine ait pû jufqu'ici répondre en pareil cas, produit néceffairement une erreur de feize toifes?

La différence de frx toifes par degré, qui réfulte de l'examen précédent, ne fert donc qu’à prouver combien les erreurs qu'on peut commettre dans la mefure géodéfque d'une Méridienne 
100 MESURE DES TROIS PREMIERS

tirent peu à conféquence, fur-tout quand on opère avec autant de précautions que nous en avons apportées; \& combien il feroit à defurer quill en fût de même des erreurs auxquelles on eft expofé dans la miefure aftronomique : c’eft-là une réflexion qui fe préfente fans cefle, \& elle a été faite par tous ceux qui ont un peu médité fur cette matière. Au refte, je n'ai employé dans mes Tables de Triangles que le calcul direet fait fur la mefure de la Bafe d'Y arouqui; afin que toutes les parties de ce calcul euflent une dépendance mutuelle, \& que l'on pût voir ce que les petites erreurs accumulées dans une fuite de 32 Triangles produiroient de différence fur le dernier côté. Je n'ai regardé le calcul fait par les deux Bafes, que comme un moyen naturel 'de corriger la longueur de la Méridienne, \& j’en ai fupprinzé tout le détail pour éviter la prolixité.

Je me fuis un peu étendu fur cette équation à la mefure de la Méridienne, par deux raifons; la première, que la chofe appartenoit très-directement à mon fujet, puifqu’il étoit queftion du changement qu'il y avoit à faire à la première valeur du degré conclu de mes opérations; la feconde, que j’ai eu lieu de croire que je ne ferois pas prévenu par $\mathrm{M}$. Bouguer fur ce point, comme je pourrai l'avoir été fur beaucoup d'autres; ayant jugé par ce qu'il a publié l’année dernière dans les Mémoires de l'Académie I 744 (page 287) qu'il ne difcutoit point cette queftion, \& ne faifoit point de correction à la longueur de fa Méridienne conclue : \& en effet il pourvoit d'autant plus s'en difpenfer, quill n'a trouvé entre fon calcul \& la mefure actuelle de la longueur de notre feconde Bafe que deux ou trois pieds de différence; au lieu que par le mien j’ai trouvé une toife, \& que j’ai voulu voir ce qui 
s'enfuivroit, en laiffant fubfufter entièrement cette différence, quoique j’euffe pû la diminuer de moitié.

\section{A R T I C L E XX VII.}

Détermination de la longueur de l'arc compris entre les deux Obfervatoires, au Nord \& au Sud de la Méridienne.

$\mathrm{P}_{\mathrm{A} \text { R la Table des diftances des Signaux à la Méridiemne }}$ \& à fa Perpendiculaire, qui fe croifent au centre de la Tour de la Mercy de Quito, on a trouvé (article XIX) que Ia fomme des diftances des Signaux de Cotche/qui \& de Chinan à cette Perpendiculaire, étoit de $177^{800} 7^{\mathrm{t}}, 87$ : cette diftance étant réduite au niveau de Carabourou. Telle eft la longueur de la Méridiemne, trouvée par le calcul; mais il y. a plufieurs corrections à y faire, \& plufieurs équations à y. appliquer, pour la réduire à la longueur vraie de l'arc du Méridien, compris entre les Parallèles à l'Équateur, qui paffent par les obfervatoires de Cotchefqui \& de Tarqui, puifque ces obfervatoires étoient fitués à quelque diftance des Signaux:

Premièrement, il y a I 8 toifes à retrancher de la longueur totale, pour la correction expliquée dans Pârticle précédent.

Secondement, il faut ajoûter 25 toifes, pour réduire le point du Signal de Cotchefqui au centre de l'obfervatoire de même nom; favoir, I $\circ \frac{1}{2}$ toifes, pour la quantité dont le lieu où répondoit le centre de notre Secteur à Cotchefqui en I 74\%, étoit plus feptentrional que le Signal, \& $14 \frac{1}{2}$ toifes, dont le Secteur de M. Bouguer étoit encore plus reculé vers le Nord. lors des obfervations fimultanées à la fin de $1742, \&$ al

$\mathrm{P} i i j$ 


\section{IO2 MESURE DES TROIS PREMIERS}

commencement de 1743 , qui font celles dont je tirerai la valeur du degré.

Troifièmement, le Signal de Chinan, terme aufral de la Bafe de Tarqui, étoit plus fud que le lieu où nous obfervâmes à Tarqui, de 8,6 toifes \& demie; ainfr que nous l'avons déterminé géométriquement avec beaucoup de précifion, par un Triangle formé exprès, dont un côté étoit une portion mênıe de la Bafe de Tarqui, actuellement nefurée : il faut donc fouftraire cette quantité de la diftance précédemment trouvée.

Enfin, il y a encore environ huit toifes à retrancher, pour Ia quantité dont la Perpendiculaire tirće de l'obfervatoire de Tarqui fur le Méridien de Quito, sécarte du Parallèle de Tarqui fur la diftance de 3 I 344 toifes, dont cet obfervatoire eft éloigné de çe Méridien vers l'occident. Cette dernière correction eft la feule qui ait befoin d'être expliquée.

Planche I,

Soit $P Q p$ le Méridien de Quito : foit $Q$ la Tour de la fig. 7 .

Mercy, par où je fais paffer le Méridien de cette ville : foit $C$ notre obfervatoire feptentrional Cotchefqui, \& $P C p$ fon Méridien : foit $T$ notre obfervatoire auftral Tarqui, \& $P T p$. le Méridien de cet obfervatoire. Cotche fqui, lieu de nos obfervations feptentrionales, n'étant éloigné de l'Équateur $E A$ que de deux minutes, la Perpendiculaire $C H$, tirée de Cotchefqui fur le Méridien de Quito, fe confondra avec le Parallèle de Cotchefqui; mais Tarqui étant éloigné de 3 degrés 5 minutes de l'E'quateur, la Perpendiculaire $T I$, tirée de l'obfervatoire $T$ fur le Méridien $P Q I$, sécarte du Parallèle $T K R$, de la quantité $I K$, qui eft l'excès de l'hypothénufe $T p$ du Triangle fphérique $p$ T I fur le côté $p$ I du même Triangle, dans lequel on connoît trois chofes: l'hypothénufe $T p$, complément de ET latitude 
de Tarqui; l'angle droit $T$ I p oppofé à thypothénufe; \& le côté $T 1$, ou la Perpendiculaire tirée de $T$, obfervatoire de $T a r-$ qui, fur la Méridienne de Quito, laquelle eft de 3 I 344 toifes*, \& peut être prife pour un arc de grand cercle, ou même de l'Équateur à caufe de fa proxinnité. On peut auffi réduire $F I$ en degrés \& minutes, fur le Parallèle de fa latitude $3^{\text {d }} s^{\prime}$; ce qui donnera. l'angle $T p I$ au Pole, \& un nouveau moyen de réfoudre le Triangle. Enfin on trouvera que $K l$, ou $T p-p I$ eft égal à $\&$ toifes, qu'il faut retrancher, comme nous l'avons dit, de la longueur de l'arc mefuré, lequel, toute réduction faite, fera de 17695 o toifes.

Voici le calcul de toutes ces différentes équations.

Somme des diftances calculées des Signaux de Cotchefqui \& de Chinant, à. Ia Perpendiculaire au Méridien de la Tour de la Mercy de Quito, trouvée art. XIX \& \& réduite au niveau du plus bas Signal.... $17780.7^{\mathrm{t}}, \varepsilon_{7}$

E'quation fouftractive de $\frac{-1}{x \circ \overline{0}} \overline{0}$, expliquée articles

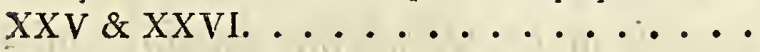

Donc, diftance corrigée des Signaux de Cotchefqui \& de Chinan, mefurée parallèlement à la Méridienne..

Ajoûtez pour la quantité, dont le centre du Secleur en 1740 à Cotchefqui étoit plus nord que le Signal...

Plus, pour la quantiré, dont à la fin de 1742 \& au commencement de 1743 , lors des obfervations fimultanées, Ie Secteur étoit plus' nord qu'en 1740 ...

Retranchez, à caufe que le Signal de Chinan étoit

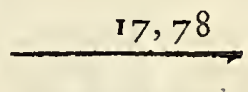

177790,09

$$
10,56
$$

$$
\frac{14,50}{177^{815}, 15}
$$

$$
\frac{856,71}{176958 i, 44}
$$

* Le Signal de Chinan, terme auftral de la Bafe de Tarqui, eft, par la i re Table art. XVIII, $32517^{t}, 68$ à l'occident du Méridien de Quito : d'où ôtant $1173^{t}, 67$, dont l'obfervatoire de Tarqui eft plus oriental que le Signal de Chinan par le calcul mentionné ( page précéd.), il refte 31344 toifes pour la différence des Triangles du Méridien de Quito \& de l'obfervatoire de Tarqui. 
IO4 MESURE DES TROIS PREMIERS DECRÉS, \&O

Somme des diftances des deux obfervatoires de Cotchefqui \& de Tarqui à la Perpendiculaire au Méridien de Quito . . . . . . . . . . 1 176958t,44

Otez $K I$, écart de $T I$, Perpendiculaire tirée de I'obfervatoire de Tarqui à la Méridienne de Quito. ou quantité dont $T I$ s'écarte fur une diftance de 3 I 344 toifes du Parallèle $T K R$ de Tarqui ... 7,97

Diftance des Parallèles des deux obfervatoires de Cotchefqui \& de Tarqui, réduite au niveau de Carabourou, 1.226 toifes au deffus du niveau de la ner. . 1 176950,47

II refteroit à réduire cette diftance au niveau de la mer: mais comme cette réduction n'eft importante que pour ia valeır du degré, je remets à la faire après la déterminationi de l'amplitude de l'arc mefuré du Méridien, ce qui fera le fujet de la Seconde Partie; \& je me contente de tirer de tout ce qui a été expofé dans la première, la conclufion fü-: vante.

La longueur totale de la Méridienne, mefurée géométriquement, \&' réduite au niveau du plus bas de nos Signaux, 1226 toifes au deffus du niveau de la mer, eft donc, toute réduction faite, de 17695 o toifes.

Fin de Ia première Partie. 


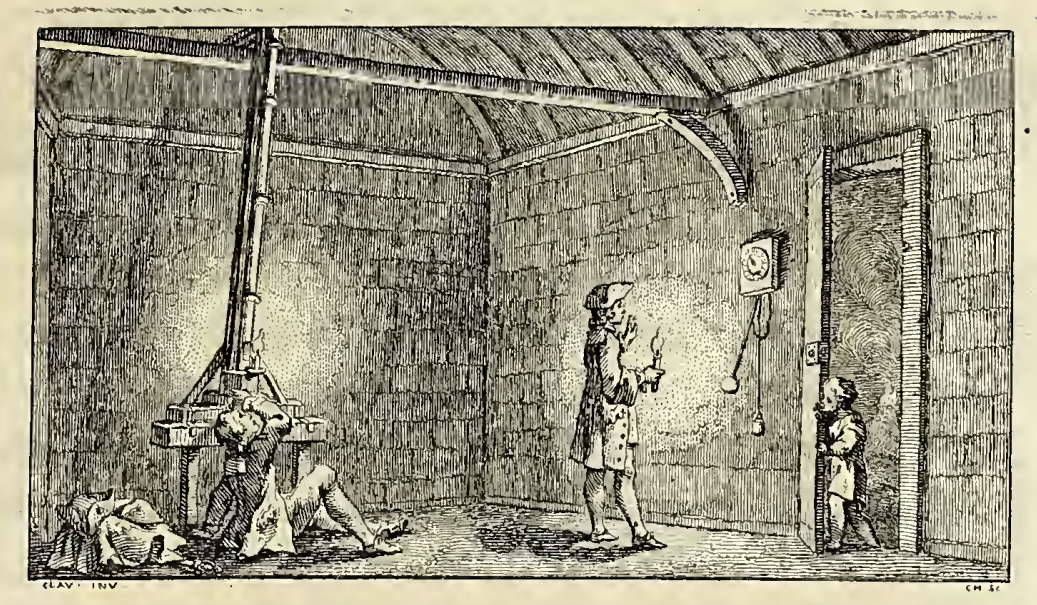

\section{E S U R E}

D E S

TROIS PREMIERS DEGRE'S

DU ME'RIDIEN .

$A U$ DELA DE L'E'QUATEUR.

\section{SECONDE PARTIE.}

MESURE ASTRONOMIQUE

$D E L A R C$ DU ME'RIDIEN, O U

DETERMINATION DE LAVALEUR DE L'ARCCE'LESTE

Qui répond à la Mefure géométrique.

P R Ès avoir déterminé, par des mefures actuelles \& par
le fecours de la Trigonométrie, la longueur d'un Arc du Méridien terreftre, il refte à connoître l'amplitude de cẹt 
Arc; c'eft-à - dire, quelle portion il eft de la circonférence de la Terre, ou combien il contient de degrés, de minutes, \& de fecondes.

L'Aftronomie feule nous en fournit les moyens, \& le plus fimple eft de faire aux deux extrémités de l'Arc, dont la longueur eft déjà connue par les mefures trigonométriques, lobfervation de la diftance de quelqu'étoile au zénith. Il eft évident que la différence des deux diftances obfervées, ou leur fomme, fi l'étoile eft entre les deux zéniths, fera la valeur de l'Arc du Méridien, compris entre les deux obfervatoires.

C'eft ainf que nous avons déterminé l'åmplitude de notre Arc, par un grand nombre d'obfervations réitérées à Tarqui, à Cotchefqui, \& même à Quito. Cette feconde Partie eft deftinée à rendre compte de ces obfervations, \& à en tirer les conféquences, quant à la valeur du clegré du Méridien.

\section{A R T I C L. E I.}

De l'ancien Secteur apporté de France; des changemens. qui $y$ furent faits pour le rendre propre aux nouvelles obfervations.

AU mois de Mai 1739, dans le temps que nous étions prêts de terminer notre Mefure géométrique, à laquelle les trois Académiciens avoient travaillé conjointement \& d'un commun accord, M. Godin déclara qu'il étoit réfolu de faire à part fon obfervation aftronomique, avec un nouvel inftrument d'un plus grand rayon quie celui que nous avions apporté 
de France, \& qui nous avoit fervi en $1736 \& 1737$ à l'obfervation de l'obliquité de I'Écliptique.

Nous reftâmes, M. Bouguer \& moi, en poffeffion de l'ancien Secteur de I 2 pieds de rayon, lequel nous parut d'une grandeur fuffiante pour déterminer l'amplitude de l'arc du Méridien dont nous avions mefuré la łongueur. Nous convîmmes feulement de faire à cet inftrument les changemens néceffaires \& convenables, pour corriger les défauts que nous avions remarqués dans fa conftruction \& fon ufage, en obfervant les Solftices.

II étoit à propos de commencer par fupprimer le limbe de 30 degrés, qui déformais n'étoit plus qu'un poids inutile, $\&$ de lui fubftituer un nouveau limbe qui pût contenir 4 ̀े $\varsigma$ degrés; cet arc étant fuffifant pour mefurer les diftances au zénith des étoiles que nous nous propofions d'obferver.

Notre première attention fe porta enfuite à éviter de tracer fur ce limbe des divifions en degrés \& en minutes, opération toûjours fujette à une grande incertitude, lors même qu'elle eft pratiquée par l'Artifte le plus habile. C'eft à quoi nous réufîmes, guidés par les réflexions fuivantes. Au lieu du grand appareil de cercles, de lignes \& de points, qu'exige $1 a$ graduation ordinaire d'un inftrument d'Aftronomie, nous n'avions befoin dans le cas préfent, où nous ne cherchions qu'une diftance au zénith, que d'un feul arc terminé par deux points. La diftance verticale de l'étoile que nous étions convenus d'obferver étoit déjà à peu près connue par les Quarts-decercle ordinaires, \& par la feule carte de nos Triangles : ainf nous pouvions, parmi les arcs un peu plus grands ou un peu plus petits que celui qui mefuroit la diftance de l'étoile au 
zénith, choifrr l'arc dont la corde feroit une partie aliquote 'du rayon. Quant à la petite quantité en plus ou en moins, dont cet arc différeroit de la vraie diftance verticale cherchée, le Micromètre nous donnoit un moyen facile de la mefurer. Tel eft l'efprit de la méthode qui nous a mis en état de nous paffer d'une divifion en degrés \& minutes; \& même de fuppléer avec avantage à cette graduation. Je détaillerai ailleurs le procédé \& l'opération.

Ce ne fut qu'à Cuenca, vers la fin d'Août 1739 , \& après avoir terminé notre mefure de la Bafe de Tarqui, que nours penfâmes férieufement à la conftruction du nouveau Secteur, en mettant en exécution les différentes idées qui s'é toient prélentées, \& qui depuis plufieurs mois faifoient le fujet ordinaire de nos converfations dans les intervalles de notre travail, pour la mefure des angles de la Méridienne. Il fut d'abord queftion de changer l'ancienne fufpenfion de cet inftrument. En 1736 \& 1737 , lors de l'obfervation de l'obliquité de l'Écliptique, il n'étoit porté que par un genou monté fur un pied de Quart-de-cercle ordinaire, \& qui n'avoit aucune proportion avec la grandeur d'un rayon de 12 pieds. Il y avoit fur cela deux partis à prendre: l'un de rendre le Secteur mobile fur le pivot d'un axe vertical de douze pieds, $\&$ cet axe ne pouvoit guère être que de bois dans un pays où le fer eft précieux : l'autre, de fufpendre l'inftrument par le centre même de l'arc, de la manière qui fera expliquée dans l'article fuivant. Je fus d'avis de domner la préférence à ce dernier moyen, comme le plus facile; peut-être n'eft-il pas le plus fûr : quoi qu'il en foit, il fut adopté pour lors.

M. Bonguer fe chargea de conduire dans l'exécution le 
freur Hugo, notre Horloger, très-capable déjà par lui-même de conftruire, \& même d'imaginer un nouvel inftrument. J'affiftai dans les commencemens deux ou trois fois à fon travail; je hhâardai même quelques avis: mais ayant jugé qu'en pareil cas la multitude des confeils pouvoit être plus préjudiciable qu'utile, je réfolus dès ce moment de m'abftenir d'en donner; \& je me trouvai bien-tôt après dans limpoffibilité de partager ce foin avec $M$. Bouguer. Nous nous vîmes alors expofés à un danger plus preffant que celui de nous tromper de quelques fecondes. Non feulement nous courûmes tous rifque de la vie dans l'émeute populaire du 29 Août 1739, dont la Relation a été publicé*; mais les auteurs du tumulte cherchant à fe juftifier, attaquèrent notre honneur, \& je me trouvai obligé de le défendre. Les procédures judiciaires en divers. Tribunaux, les feules lettres à l'Audience Royale de Quito, au Gouverneur de la Province, au Viceroi, \& celles qu'il me fallut écrire dans le même temps en France, ne m'euffent pas laiffé affez de temps pour fuivre de près la conftuction de notre inftrument, ni les préparatifs de notre obfervation aftronomique; mais rien n’étoit moins néceffaire, puifque M. Bouguer s'en étoit chargé, \& que je m’en rapportois plus à lui qu’à moi - même.

Il refte à donner la defcription de notre nouveau Secteur.

La planche III le repréfente en perfpective tout monté, \& Plancheili: tel que je le deffnai d’après nature, dans le temps de nos premières obfervations à Tarqui en I 739. Je prêtai dans le même temps mon deffein à M. Verguin, qui en fit une copie poux M. Bouguer.

* Lettre fur l'émeute populaire excitée à Cuenca au Pérou contre les Acam. démiciens, \&c. Paris, MI, DCC XLVI. 


\section{A R T I C L E II.}

\section{Defcription du Secteur.}

Notres Secteur, dans fa nouvelle conftuction, n'étoit plus compofé que de trois pièces principales; d'un limbe de cuivre; d'un rayon formé d'une barre ou règle de fer qui joignoit le limbe au centre, \& d'une pièce qui portoit le centre \& fe terminoit en un fegment de fphère ou portion de boule par laquelle l'inftrument étoit fufpendu.

$A B$ eft une règle longue de deux pieds, large d'un pouce $\&$ demi, \& de deux à trois lignes d'épaiffeur. Cette règle étoit de cuivre, \& appliquée avec des cloux de même matière, rivés fur une bande de fer $a b$, garnie par derrière d'une règle de chan a b. On s'étoit difpenfé de donner au limbe une courbure circulaire; mais fa largeur verticale étoit fuffifante pour contenir la courbure d'un arc de cercle de feptà huit degrés. Au milieu de la bande de fer $a b$, qui foûtenoit le limbe, étoit attachée avec des tenons $\&$ des vis l'extrémité inférieure $C$ d'une règle plate de fer $C D$, large de trois pouces, épaifle de denx lignes, \& longue de douze pieds. Cette règle formoit le rayon du Secteur, \& fervoit à lier le limbe avec le centre de l'inftrument: elle eft repréfentée brifée dans Ia figure, pour éviter de donner à la planche trois fois autant de hauteur qu'elle en a, comme l'en̂t exigé fans cela, la proportion des parties du deffein. La règle $C D$ étoit de deux pièces, lefquelles, au milieu de la longueur du rayon, fe recouvroient l'une l'autre de quelques pouces, \& s'uniffoient par le moyen de plufreurs tenons \& clavettes chaflées à force: cette 
même barre $C D$ avoit auffi une règle de chan $J J J$ qui lui étoit adoflée dans toute fa longueur, pour la contenir \& l'empêcher de s'arquer.

L'extrémité fupérieure du même sayon s'élargiffoit vers $D$, \& recevoit fur fa face antérieure, aplatie \& limée en retraite, une pièce de cuivre $E F G$, avec laquelle elle étoit aflemblée par trois fortes vis e e e. Un peu plus haut, la pièce de cuivre étoit percée en $I$ d'un trou difpofé pour recevoir un cylindre de même matière, fait au tour, \& qui fervoit de centre à l'inftrument. Ce centre ne différoit en rien de celui d'un Quart-decercle ordinaire. La même pièce de cuivre $E F$ fe prolongeoit en $G, \&$ prenoit une forme conique qui fe terminoit par le haut en un fegment de fphère, ou portion de boule, Jaquelle étoit embraffée par un carcan ou collier $K$, pratiqué à l'extrémité d'une potence de fonte $K H$, avec fon fupport $L$ : le tout fermement arrêté à une poutre de l'obfervatoire. La partie fupérieure du carcan $K$ étoit évafée, pour recevoir \& Iaiffer rouler librement la demi-boule qui y étoit engagée. Cette demi-boule, qui faifoit les fonctions du genou dans les planchettes d'Arpenteur, fervoit de point de fufpenfron à l'inftument; \& par fon moyen il pouvoit facilement fe tourner \& s'incliner en tout fens.

La bande de fer $a b$, fur laquelle le limbe $A B$ étoit rivé, portoit à fa partie inférieuse deux pièces faillantes $M M$, ou tenons plats qui fervoient à retenir le Secłeur dazs la fituation qu'on vouloit Jui donner. Ces deux tenons étoient reçûs dans les fentes ou coulifies de deux taffeaux de fer $m m$, enchaffés dans une pièce de bois $O O$, \& pouvoient y être mûs parallèlement au plan du limbe par le moyen de deux vis $n n$, 


\section{2 MESURE DES TROIS PREMIERS}

qui agiffoient en fens contraire; \& qui, en conduifant doü cement l'un ou l'autre des deux tenons $M$, faifoient mouvoir le Secteur fur fon centre dans le plan du limbe, \& donnoient toute la facilité poffible de changer l'inclinaifon de l'inftrument, d'une auffi petite quantité qu'on vouloit.

La pièce de bois $O O$, à laquelle étoient fixés les deux tafleaux à couliffes $m m$, étoit couchée fur un banc folide $Q Q$; dont les pieds étoient enfoncés en terre de quatre pieds de pro: fondeur; aux deux bouts de ce banc étoient arrêtés deux cranpons de fer $R R$ en forme de double équerre ou d'étriers, fous lefquels paffoit la pièce qui portoit les barres des taffeaux. Ces étriers étoient garnis chacun de trois vis, une en avant $S$, une en arrière $s$, \& l'autre en deffus $t$ : les premières fervoient à mou: voir doucement la pièce de bois, parallèlement à elle-même; en avant \& en arrière, fuivant qu'on en avoit befoin pour: caler l'inftrument. Les vis $t$ t d'au deffus de chaque étrier fervoient à la comprimer fur le banc, \& à la fixer dans la fituation qu'on lui avoit une fois donnée : les deux premières vis $\mathcal{S}$, deftinées principalement à la faire changer de direction; concouroient auffi à l'affujétir dans celle où on vouloit la fixer.

La lunette $X Y$ étoit embraffée par des fourchettes de fer rivées fur le rayon $C D$. $W$ repréfente le Micromètre adapté à la lunette pour mefurer les minutes \& fecondes, dont Ia diftance de l'aftre au zénith étoit, ou moindre, ou plus grande que la moitié de l'arc * a w tracé fur le limbe. Enfin $P$ défigne le poids fufpendu librement par un cheveu ou un fil de Pite. $I E D C$ au centre $I$ de l'inftrument.

* On expliquera dans l'article fuivant pourquoi l'arc tracé fur le limbe śtoit double de la diftance de l'aftre au zénith. 


\section{A R T I C L E III.}

De lObjervatoire de Tarqui. Détermination de la valeur des parties du Micromètre. Préparatifs communs à toutes nos obfervations de l'amplitude de l'Arc.

LE 29 Septembre 1739, je me rendis de Cuenca à Tarqui, où $M$. Boutguer \& moi choisîmes le lieu qui nous parut le plus propre pour faire notre obfervation aftronomique. C'étoit une grande pièce au raiz-de-chauffée, nouvellement bâtie, \& deftinée à faire ta chapelle d'une maifon de campagne à cinq lieues au Sud de Cuenca, \& à un quart de lieue du terme auftral de la feconde Bafe que nous venions de meGurer.

Le lendemain 30 , nous examinâmes la valeur des parties du Micromètre de la lunette de douze pieds qui devoit être appliquée au Secteur. Pour cet effet nous nous tranfportâmes à une extrémité de la Bafe déjà mefurée; \& ayant fait placer à l'autre extrémité deux mires, éloignées l'une de l'autre de 80 pieds, fur une ligne qui faifoit avec ta Bafe un angle droit, \& qui, par conféquent, à la diftance où nous étions de '5260 toifes, étoit. le finus d'un angle de $8^{\prime} 43^{\prime \prime} \frac{2}{3}$, nous trouvâmes que cet angle répondoit à y 196 parties du Micromètre, felon l'eftime de $M$. Bouguer, \& à I 193 felon ta mienne. Nous prîmes le milieu entre ces deux nombres, \& nous conclûmes que mille parties étoient la corde d'un angle de $7^{\prime}$ I $8^{\prime \prime} 24^{\prime \prime \prime}$. Sur ce fondement, je fis une Table de la 
valeur des parties du Micromètre, \& elle m'a fervi depuis pour toutes les obfervations où la même lunette a été employée, tant cette année, que les fuivantes. On voit par cette Table, qu'une partie du Micromètre répondoit à 26 tierces; c'eft-à-dire. quil falloit prefque trois parties pour faire une feconde; $\&$ cette quantité étoit, comme on vient de le voir , toute la différence que nous avions trouvée fur un angle de près de neuf minutes, M. Bouguer \& moi, avec des yeux fort diverfement conformés.

Le premier Ođtobre, on commença à difpofer la charpente qui foûtenoit le toit de notre Obfervatoire, pour recevoir les pièces de fer \& de fonte deftinées à fufpendre le Secteur. Je laiffai M. Bougucr à Targui occupé de ces préparatifs, \& jallai le 2 à $C$ uenca, pour les affaires dont j’ai parlé, \& pour faire finir le limbe de notre Secteur. Le fieur Hugo le porta le 4 à Tarqui, où jarrivai le 6 à midi, \& où je trouvai J'inftrument monté. Tout étoit prêt de notre part pour l'obfervation, lorfque le Ciel fe couvrit de nuages, \& fe déroba pendant un affez long temps à nos regards.

L'arc que M. Bouguer avoit tracé s'étoit trouvé, vû l'examen qu'il en avoit fait après l'avoir terminé par deux points, plus grand que la dix-huitième partie du rayon, d'une petite quantité que $M$. Bonguer avoit évaluée.

Le 8, aidé de M. Verguin, je remefurai ce même arc, \& je vérifiai le rapport de fa corde au rayon; non que jeuffe le moindre fcrupule fur la jufteffe d'une opération où M. Bous guer avoit donné tous fes foins, \& dans laquelle même il avoit été fecondé par M. Verguin, le fieur Hugo, \& un autre aide adroit \& intelligent; mais parce que me trouvant chargé de ba 
même commiffion que M. Bouguer, je me crus obligé de m'afút rer par moi-même de tous les faits dont j’étois refponfable, pour en pouvoir dépofer comme témoin oculaire. Je trouvai l'excès du rayon fur la corde de l'arc, répétée dix-huit fois, le même, à $\frac{1}{12}$ de ligne près, que $M$. Bonguer l'avoit eftimé, \& nous prîmes un milieu entre nos deux déterminations. Les jours fuivans, nous travaillâmes à régler la Pendule par des hauteurs correfpondantes, \& à déterminer, puis à vérifier, par plufieurs obfervations, une Méridienne qui étoit marquée par un filet de cheveux noués bout à bout, tendu d'un mur de l'Obfervatoire ¿̀ l'autre, dans une longueur de plus de 20 pieds.

Les deux extrémités de ce fil, chargées chacune d'un poids fuffifant pour tendre le cheveu, portoient fur deux crampons de fer, où l'on avoit fait un trait de lime, qui fervoit de repaire pour placer le fil toûjours au même endroit: le cheveu ainf tendu dans l'alignement de la Méridienne, fervoit à diriger le limbe du Secteur dans le plan du Méridien; il fuffíoit pour cela de rendre le limbe parallèle au fil. Cette opération fe faifoit par le moyen des vis de régie $s, S$, qui fervoient à changer Ia direction du limbe; \& le cheveu le rafoit à une fi petite diftance, qu'ón pouvoit, à la vûe feule, juger du parallélíme. Cependant, pour nous en affurer avec plus de précifron, nous nous fervions d'une échelle de lignes parallèles très-fines, tirées ł̀ un quart de ligne de diftance les unes des autres, \& tracées fur le dos d'une carte à jouer. On préfentoit le côté de cette carte alternativement aux deux extrémités du limbe, immédiatement au deffous du cheveu; \& on examinoit à laquelle des lignes tracées il répondoit. On ne pouvoit guère fe tromper d'une demi-divifion, ou de $\frac{I^{\prime}}{4}$, ce qui, vûla longueur du limbe de 


\section{MESURE DES TROIS PREMIERS}

25 pouces, nous affuroit de fa direction, au moins à $I \frac{1}{2}$ près.

Comme la manière de tracer l'arc fur le limbe, \& de le vérifier, fut à peu près la même dans cette première obfervation, \& dans les autres poftérieures; que le procédé en eft fort fimple, \& quill n'exige point de divifions du limbe de l'inftrument en degrés \& en minutes: je crois qu'il eft à propos de l'expofer ici avec quelque détail, \& de mettre ainf le Lecteur en état de juger de l'exactitude que nous pouvions nous en promettre.

\section{A R T I C L E IV.}

De l'Arc tracé fur le Secteur. Manière d'obferver la diftance d'une E'toile au Zénith, fans le fecours des divifions ordinaires.

L'Ét orl e $\varepsilon$ d'Orion, de la feconde grandeur, \& qui nous avoit déjà fervi à la vérification du Secteur par le renverfement, dans le temps de nos obfervations des folftices, nous parut la plus propre pour la mefure de l'amplitude de l'arc du Méridien.

Comme la longueur mefurée de cet arc de trois degrés étoit prefque toute au defà de l'Équateur, notre étoile, qui avoir I 24 ' de déclinaifon auftrale, fe trouvoit répondre vers le milieu de l'arc, \& à peu près à égale diftance des zéniths de fes deux points extrêmes.

Nous favions déjà auffi, foit par la mefure de nos Triangles depuis les lieux dont la latitude nous étoit connue, foit par des obfervations faites avec des Quarts-de -cercle ordinaires, que l'étoile devoit être éloignée du zénith de Tarqui d’environ 
$I^{d} 4 I^{\prime} ; \&$ par conféquent que lorfque la lumette feroit pointée

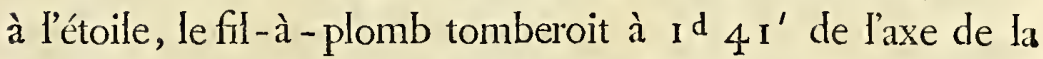
Iunette, \& qu'il tomberoit à pareille diftance du côté oppofé lorfqu'on retourneroit l'inftrument pour la vérification. Nous n'avions donc befoin, pour obferver Ia diftance de l'étoile au zénith dans les deux fituations du Secteur, que d'un arc de $3^{d} 22^{\prime}$, c'eft-à-dire, double du précédent. Or en ouvrant les Tables des finus, on voit. quil ne manque que is fecondes à cet arc pour que fa corde foit précifément la dixfeptième partie du rayon ; \& ce petit excès pouvoit facilement fe mefurer avec le Micromètre. Ainf toute la difficulté fe réduifoit à trouver le moȳen de tracer exactement fur le limbe de notre Secteur un arc de $3^{d} 22^{\prime}$ i $5^{\prime \prime}$, ou pluftôt la corde de cet arc; c'eft-à-dire, une ligne égale à la dixfeptième partie du rayon *

Ceux qui ne fe font pas contentés d'opérel fur le papier , n'ignorent pas combien il eft difficile de divifer trés-exactement une ligne donnée en un certain nombre de parties fans aucun refte: ils favent qu'on ne peut fe flatter d'y réufir que par un tâtonnement long \& pénible; \& d'autant plus long \& plus difficile, que le nombre des parties de la divifron eft plus grand. Heureufement nous n'étions pas aftreints à faire le rayon de notre inftrument précifément d'une certaine longueur, \& la largeur de notre limbe permettoit de donner à ce rayon un pouce de plus ou de moins. C'eft-là ce qui lève toute la difficulté de l'opération; au lieu de tâtonner longtemps pour trouver, par la divifion d'un xayon domné, la

* Nous nous fervîmes, pour les premières obfervations, de la I $8^{e}$ partie du rayon, \& enfuite de la $17^{c}$ : on en verra bien-tôt la raifon. 


\section{MESURE DES TROIS PREMIERS}

valeur d'une corde qui en foit une certaine partie aliquote, on peut procéder fùrement, en prenant pour corde une grandeur approchée de celle qu'on cherche, \& en la multipliant le nombre de fois requis. Voici le détail de la manière dont nous avons toûjours fait cette opération.

On ouvroit un compas à arc-de-cercle, ou, en termes d'Horlogerie, un compas d'arrêt, fait exprès pour cet ufage, d'une quantité telle, qu'étant portée dix-fept fois fur le rayon depuis le centre, elle dût, à la dernière fois, tomber à quelque point de la furface du limbe. Par exemple, dans le cas dont il eft ici queftion, on ouvroit le compas de 8 pouces 6 lignes; qui, multipliés par 17 , font I 2 pieds 6 lignes. Cette ouverture de compas une fois déterminée, on la rendoit invariable par le moyen d'une vis deftinée à cet effet.

On portoit cette même ouverture de compas dix-lept fois, Je long d'une foie tendue fur une règle de bois de plus de douze pieds de long, bien dreffée \& couchée horizontalement; \& afun que les pointes du compas n'enfonçaffent pas dans le bois, la règle étoit garnie de petites plaques de métal d'égale épaiffeur, pofées aux diftances convenables, pour recevoir ces pointes. Cette opération ayant été répétée jufqu'à ce qu'on fe fût bien affuré du point où tomboit le compas après le dixfeptième intervalle parcouru, fa diftance comprife entre les deux points extrêmes des dix-fept intervalles, déterminoit la la longueur précile du rayon du Secteur, \& on mettoit à part le compas d'arrêt ouvert de la $17^{\mathrm{e}}$ partie de cette longueur; alors on élevoit la règle verticalement contre un mur, \& avec un compas à verge de douze pieds, d'un Lois lèger \& bien fec, on prenoit exactement entre les deux pointes de ce compas 
dans cette fituation verticale, la diftance des deux points extrêmes marqués fur la règle. Tout cela, vû la hauteur de l'inftrument, qui étoit de douze pieds, exigeoit, comme on voit, l'appareil d'une échelle aflez haute, \& le concours de deux perfonnes intelligentes. La mefure du rayon étant bien prife fur la règle entre les deux pointes du compas à verge, dont l'une pouvoit recevoir un mouvement très-doux par le moyen d'une vis, un des deux opérants tranfportoit ce compas toûjours dans la même fituation verticale, \& pofoit une de fes pointes fur le centre du Secteur tout monté, en foutenant le compas; tandis que le fecond promenoit légèrement l'autre pointe fur le limbe, \& traçoit un arc de cercle. Enfin on limitoit cet arc par deux points, qu'on marquoit à égale diftance de part \& d'autre du rayon avec l'ouverture du compas d'arrêt, laquelle étoit reftée en dépôt exprès pour cela.

Tout ceci étant bien exécuté, il eft évident que fi l'on place le Secteur dans le plan du Méridien, \& $\mathrm{f}_{\mathrm{I}}$ on l'incline en forte que le fil-à-plomb tombe fur un des points extrêmes de l'arc tracé, que je fuppole ici de $3^{d} 22^{\prime}$ I $5^{\prime \prime}$, la lunette fe trouvera dirigée à $I^{d} 47^{\prime} 7^{\prime \prime} \frac{1}{2}$ du zénith; c'eft-à-dire, à une diftance égale à la moitié de l'arc; \& que par conféquent l'étoile paffera dans la lunette, \& ne paroîtra éloignée du fil horizontal que de la petite quantité dont fa diftance au zénith fẹra ou plus petite ou plus grande que la moitié de l'arc tracé: quantité qui dans l'un \& dans l'autre cas peut fe mefurer exactement par le Micromètre.

J'ai fuppofé, pour plus de fimplicité, que l'axe optique de la lunette répondoit précifément au milieu de l'arc; mais cette circontance n'eft pas néceflaire, pourvû guion retourne l'inf- 
trument, comme nous l'avons toûjours pratiqué : car alors Ia diftance au zénith eft autant augmentée dans une des lituations du Secteur, qu'elle eft diminuée dans l'autre; \& ta moitié de la Somme des deux diftances eft la vraie.

Nous avons quelquefois obfervé la diftance de la mêne étoile au zénith fur deux différens arcs, tracés par la méthode précédente; ce qui faifoit l'effet de deux inftrumens différens. A Tarqui, par exemple, nous oblervâmes les premiers jours avec un arc de $3^{d}$ I I' $5^{\prime \prime} \frac{1}{5}$, dont la corde étoit à peu près la dix-huitième partie du rayon, \& qui avoit été tracé par M. Bouguler d'abord en arrivant, avant que de s'être bien affuré de la latitude du lieu. Pour faire paffer l'étoile plus près du centre de la lunette, nous traçâmes depuis un nouvel arc de $3^{d} 22^{\prime}$ I $5^{\prime \prime}$, dont la corde étoit exactement la dix-feptième partie du rayon.

C'eft ainfr qu'en traçant à chaque différente diftance au zénith que nous obfervions, un nouvel arc dont la corde étoit fousmultiple du rayon, nous avons fuppléé par un moyen fort fimple, au défaut d'un inftrument auffi parfait que le Secteur de M. Graham, duquel les Académiciens, qui ont fait le voyage de Lapponie, ont eu l'avantage de fe fervir. D'ailleurs, la divifion en degrés \& minutes, dans laquelle la probabilité des erreurs croît à proportion de la difficulté, \& du nombre des opérations, nous étoit inutile. Nous n'avions befoin que d'un feul arc à chaque fois, \& notre manière de le tracer portoit avec elle fa vérification. J'ignore fi ce moyen de fe fervir d'une partie aliquote du rayon, pour tenir lieu de graduation fur un inftrument, a été pratiquée en d'autres occafrons. C'eft à M. Godin que jen ai oü parler le premier, avant 
notre départ de France. M. Cafini de Thuryl'a aufi propofée en I736. L'ufage de cette méthode femble d'abord borné à la mefure des arcs dont les cordes font fous-multiples du rayon; Mém. de l'Académie, 1736, page mais il peut s'étendre beaucoup plus loin à l'aide du Micromètre, dont l'application aux inftrumens aftronomiques eft dûe à $M$. Ie Chevalier de Louville, \& eft, fans contredit, une des plus utiles inventions de l'Aftronomie moderne.

\section{A R T I C L E V.}

Des differentes obfervations aftronomiques faites dans la Province de Quito, pour déterminer l'amplitude de l'arc du Méridien.

JE ne rappellerai point ici l'hiftoire de nos obfervations aftronomiques, deftinées à conclurre l'amplitude de l'arc du Méridien, ni les motifs qui nous ont engagés à les répéter à diverfes reprifes, en différens temps, \& en différens lieux. Je les ai fuffifamment expliqués dans l'Introduction hiftorique, qui eft à la tête de cet ouvrage.

Je ne deftine cet article qu'à expofer l'ordre, le temps \& le lieu de ce grand nombre d'obfervations dont je domnerai le détail, le réfultat \& la critique dans les articles fuivans. Je ne fais ici que les mettre fommairement fous les yeux du Lecteur, pour prévenir les équivoques, \& la confufion que la multiplicité de ces obfervations pourroit occafionner.

\section{Premières obfervations à Quito en 1737.}

L'étoile \& d'Orion, qui n'étoit éloiginée du zénith de Quito que de $I^{\mathrm{d}} \mathrm{I}_{\mathrm{O}}$, nous ayant paru propre à vérifier l'erreur de 


\section{MESURE DES TROIS PREMIERS}

la pofition de la lunette de notre Secteur, après l'obfervation des deux folftices de Décembre 1736 \& Juin 1737 , nous obfervâmes, M. Godin, M. Bonguer \& moi, en Janvier \& Juillet 1737 , la diftance de cette étoile au zénith. Nous n'avions pas alors pour but de faire fervir ces obfervations à la détermination de l'amplitude de l'arc du Méridien ; cependant comme elles peuvent être employées à cet ufage, je crois devoir les rapporter ainf que les autres. Quant à leur détail, je n'en ferai qu'un article avec les autres obfervations faites ì Quito en I 740, I 74I \& I 742 .

\section{Premières obfervations au Sud de la Méridienne, i Tarqui, en 1739 .}

Nouis commençâmes le r 8 Octobre 1739 nos premières obfervations pour l'amplitude de l'arc du Méridien, au Sud de Ia Méridienne, à Tarqui, cinq lieues au delà de Cuenca, par $3^{\mathrm{d}} 5^{\prime}$ de latitude auftrale : elles durèrent jufqu'au I 3 Janvier 1740. Dans cet intervalle de temps, nous fimes trois Suites d'obfervations indépendantes l'une de l'autre. J'entends ici par Suite, un nombre fuivi d'obfervations de la même étoile, faites dans les deux frtuations du Secteur, dirigé alternativement au Nord \& au Sud; \& j’appelle Suites indépendantes, celles qui font diftingućes l'une de l'autre, par quelque changement, ou fait ou furvenu à l'inftrument, dans l'intervalle des deux Suites; foit en démoñtant le Secteur, foit en traçant un nouve arc, foit en changeant Ia fituation de la lunette, ou celle de l'objectif.

Outre l'étoile $\varepsilon$ d'Orion, nous obfervâmes alor's \& ordinairement depuis, deux autres étoiles, $\theta$ d'Antinoiis, \& a du Verfeam: 
mais comme $\varepsilon$ d'Orion eft celle que nous avons fuivie le plus conftaniment, que c'elt celle dont nous avons un plus grand nombre d'obfervations, \& la feule qui nous en ait fourni aux deux extrémités de l'arc de correfpondantes \& fimultanées, dont nous fommes convenus de déduire la valeur du degré du Méridien; je ne donnerai le détail que de celles de cette étoile.

\section{Premières obfervations au Nord de la Méridienne, à Cotchefqui, en $174^{\circ}$.}

De Tarqui nous paffâmes, M. Bougutur \& moi, à l'extrémité auftrale de la Méridienne, en un lieu appelé Cotchefqui. Nous commençâmes à y obferver le r 9 Février i 740 , \& nous cefiâmes le 25 Avril fuivant.

\section{Secondes obfervations à Quito en 1740 \& 174 I.}

M. Bouguer fit remonter à Quito le même Secteur qui nous avoit fervi aux deux extrémités de l'arc du Méridien, \& obferva en fon particulier à Quito, la diftance de la même étoile, ou des trois étoiles au zénith, au mois de Sept. 1740.

If recommença \& termina une nouvelle Suite d'obfervations au mois d'Octobre fuivant; je n'ai eu communication que du réfultat de cette Suite, non plus que de celui de Ia précédente.

J'obfervai auffi en mon particulier dans le même lieu, après avoir remefuré l'arc \& fa corde, la diftance de l'étoile $\varepsilon$ d'Orion au zénith, au mois de Novembre, \& jufquau 30 Décembre de la même année. Je ne pûs apercevoir les deux autres étoiles, qui paffoient de jour: les mauvais temps, les 
124 MESURE DES TROIS PREMIERS pluies, \& d'autres obftacles traverérent beaucoup cette obfervation, qui ne fut pas même terminée.

Le 3 o Décembre, $M$. Bouguer changea la lunette de longueur, pour l'accommoder à fa vûe, \& recommença une nouvelle Suite d'obfervations qui dura jufqu'au 2 Février $174 \mathrm{r}$.

II s'en faut I 4 à I 5 minutes que Quito ne foit à l'extrémité feptentrionale de l'arc du Méridien dont nous avons mefuré la longueur; mais la diftance entre le Parallèle de la Jatitude de Quito, \& celui de Cotchefqui, terme nord de notre Méridienne, étant connue en toiles, les obfervations de Quito peuvent être rapportées à celles qui ont été faites au Nord de la Méridienne.

Je ne mets point en ligne de compte les obfervations que je fis à Quito, pendant une grande partie de l'année i 74 I, des trois mêmes étoiles, \& de plufieurs autres, avec une lunette fixe de i 5 pieds. Le but de ces obfervations étoit feulement d'examiner les changemens de diftance au zénith, \& non les diftances abfolues, que je n'euffe pû conclurre qu'en retournant ła lunette : ce qui n'étoit pas poffible dans le cas préfent, puifqu'elle étoit fellée dans un mur avec deux bras de fer.

\section{Secondes obfervations au Sud de la Méridienne, à Tarqui, en I 74 I.}

Pendant que jobfervois à Quito les variations apparentes de ces étoiles, fuivant nos conventions, M. Bouguer retourna Tarqui au mois de Février I $74 \mathrm{x}, \&$ y fit, à différentes re. prifes, un grand nombre d'obfervations de leurs diftances au zénith : elles compofent cinq Suites différentes. Il commença le s Mars, \& funit d'obferver le 4 Décembre I 74 I. 
Troifièmes \& dernières objervations à Quito en 1742.

Je me préparai à la répétition que jallois faire, après M. Bouguer, à Tarqui, au Sud de la Méridienne, de nos anciennes obfervations de 1739 , par celles que je fis à Quito en $174^{2}$, avant mon départ pour Tarqui, de nos trois étoiles, depuis le 5 Mai jufqu'au 3 I Juillet. \& d'Orion paffoit au Méridien en plein jour; je ne perdis cette étoile de vûe, que lorfqu'elle paffa à une heure \& demie après midi, \& je la revis à dix heures \& demie dumatin, quand elle eut devancé le Soleil. Je ne ceffai d'obferver que lorfquiil me fallut démonter le Secteur pour l'envoyer à Tarqui.

Troifièmes \& dernières obfervations au Sud de la Méridienne, à Tarqui, on $174^{2}$ \& 1743 , correfpondantes à celles de Cotchefqui, \& fimultanées.

Je me rendis à Tarqui à la fin de Septembre 1742. Ce ne fut quà la fin de Novembre que je pûs commencer à y obferver utilement; je continuai mes obfervations jufques en Mars \& Avril 1743.

Secondes \& dernières obfervations au Nord de la Méridienne, à Cotchefqui, en 1742 \& 1743 , correfpondantes à celles de Tarqui, \& fimultanées.

Pendant que jobfervois feul à Tarqui en I 742, M. Bouguer répétoit au Nord de la Méridienne, avec un nouveau Secteur, les obfervations que nous y avions faites enfemble en 1740 avec celui qui me fervoit actuellement à Țarquio M. Bouguer avoit commencé à obferver à Cotchefqui dès le mois d'Août 1742 , \& il finit au mois de Janvier 1743 : 


\section{MESURE DES TROIS PREMIERS}

Dans cet intervalle de temps, nous eûmes plufieurs obfervations correfpondantes de la même étoile, faites les mêmes nuits \& à la même heure aux deux extrémités de l’arc.

Ce fera uniquement de ces dernières obfervations, faites dans le même temps aux deux bouts de la Méridienne, que je tirerai, comme nous en fommes convenus, M. Bouguter \& moi, la valeur du degré du Méridien; mais ce ne fera qu'après avoir examiné le degré de confiance que mérite chacune de nos diverfes Suites d'obfervations, \& avoir expofé les raifons que nous avons eues de rejeter les anciennes, faites fucceffivement aux deux extrémités de l'arc (fur-tout celles de Tarqui de I739) pour nous en tenir aux obfervations fimultanées de I 742 \& I 743 . Je ferai voir aufli que toutes les autres, à l'exception de celles de Tarqui en I 739, ne diffèrent guère dans leur réfultat, de celui des obfervations fimultanées, qui méritent la préférence à tous égards.

Pour mettre le Lecteur à portée de comparer facilement nos diverfes obfervations, $\ddot{j}$ en donnerai autant de Tables, que nous avons fait d'obfervations différentes; \& je réduirai chacune en particulier au temps des obfervations fimultanées, en prenant pour leur époque le premier Janvier i 743 .

Cette réduction fervira à faire mieux juger de la jufteffe des obfervations, par leur plus ou moins de conformité. On verra, par exemple, que telle obfervation qui paroiffoit différer de 8 fecondes d'une autre de la même Suite, \& firite deux mois auparavant, en diffère à peine d'une feconde après Ia réduction; \& réciproquement, que celles qui paroiffoient le mieux s'accorder, font quelquefois celles qui, réduites à la même époque, diffèrent le plus entr'elles. 
J'emploie pour cette réduction trois équations différentes.

La premịère, pour corriger le changement que caufe dans Ia hauteur de l'étoile, la préceffion des Equinoxes, qui eft l'effet de Ia révolution de l'axe de la Terre autour des Poles de l'Écliptique. Cette équation eft connue depuis plufieurs fiècles, \& tous les Aftronomes font d'accord, à très-peu près, fur fa quantité; fur-tout pour de courts intervalles de temps: je l'ai fuppofée d'un degré en 72 ans.

La feconde équation, qui eft celle qu'exige l'aberration de Ia lumière, eft dûe aux obfervations délicates, \& aux fubtiles recherches de $M$. Bradley, dont l'ingénieufe théorie, expofée dans les Tranfactions philofophiques, ann. I $728,100^{\circ} 406$, traitée par M. Manfredi dans les Commentaires de l'Inftitut de Boulogne en r 730, \& étendue par M. Clairaut dans les Mémoires de I'Académie de 1737 , eft aujourd'hui adoptée par tous les Aftronomes.

La troifième équation eft celle qui réfulte de la nutation de l'axe de la Terre; c'eft encore une découverte de $M$. Bractley, mais plus récente que la précédente. L'une \& l'autre ont été également confirmées par les obfervations de M. Ie Monnier, faites avec le Secteur de M. Graham, \& rapportées dans. łes Mémoires de l'Académie de r 745 . On peut voir l'expofé de cette nouvelle théorie dans l'extrait du Mémoire de M. Bradley par M. l'Abbé de la Caille, publié dans Jes Mémoires de Trévoux du mois d'Octobre r 748.

En réduifant, comme je l'ai fait, toutes les obfervations jour par jour, je ne laiffe aucun doute au Lecteur, qui a Lous les yeux les élémens du calcul, \& qui peut les vérifies: aifément, ainf que les conféquences que j’en tire. 
28 MESURE DES TROIS PREMIERS

La diftance apparente de l'étoile au zénith fe conclud en ajoûtant au demi-arc, qui a fervi à l'obfervation, la quantité moyenne obfervée avec le Micromètre, dans les deux fituations inverfes du Secteur. Si cette quantité eft négative, il faut la fouftraire du demi-arc, au lieu de l'y ajoûter.

\section{A R T I C L E V I.}

Premières obfervations à Tarqui, extrémité auftrale de la Méridienne, en Novembre \& Décembre 1739. \& Janvier 1740 .

JE ne puis rendre un compte plus exact de nos premières obfervations à Tarqui, ni mettre dans un plus grand jour les attentions avec lefquelles nous procédâmes dès ce premier travail, qu'en domnant ici la copie du procès verbal même que $M$. Bouguer dreffa de ces obfervations, \& quili fit certifier par un Notaire à Cuenca, auffi-tôt qu'elles furent terminées, au mois de Janvier 1740. On en jugera mieux combien de femblables opérations font délicates, \& combien il eft diffcile, en pareil cas, d'éviter l'erreur; puifque, malgré toutes nos précautions, nous ne pûmes nous en garantir dans ce premier effai.

\section{Procis verbal des obfervations faites ì Tarqui en 1739 .}

C'eft M. Bouguer qui parle.

"Nous trouvant obligés, $M$. de la Condamine \& moi, de " faire à part les obfervations aftronomiques, qui doivent ap» prendre en parties de la circonférence de la Terre, la valeur » de l'arc du Méridien, dont nous avons déjà mefuré la valeur 
en toifes; nous nous déterminâmes à faire la première de ces « obfervations dans une maifon de campagne appartenante à « N. ...... fituée dans un des enfoncemens de Ia « plaine de Tarqui, dans laquelle nous avons mefuré la Bafe « dont nous avons déjà communiqué la longueur actuelle, de « même que la.fituation, à M. Godin, en lui faifant part des « angles de tous les Triangles qui fervent à la lier avec les « autres ftations de la Méridienne. L'endroit de cette maifon, “ que nous avons choifi, eft éloigné de notre Bafe de cinq " cens trente toifes \& demie du côté de l'orient, fur une per- « pendiculaire qui rencontre la Bale à treize cens cinquante- “ trois toifes de fon extrémité auftrale. Cet endroit eft une Salle « fermée, dans laquelle nous avons fait faire un retranchement ๙ avec des nattes, afin d'être encore plus difpenlés de mettre « un garde-filet au fil-à-plomb, ou de faire defcendre, comme « nous le faifions quelquefois à Quito à l'obfervation de l'obli- « quité de l'Écliptique, le plomb dans un vafe plein d'eau. “ L'inftrument, qui eft formé de diverfes règles de fer, \& “ qui a douze pieds de rayon, fe trouva entièrement monté «. dès le commencement d'Octobre dernier. Nous avions véri- « fié, M. de la Condamine \& moi, en nous fervant de toute la « longueur de notre Bafe, la valeur des parties du Micromètre, “ que j’avois déjà examinée en particulier, en comparant le jeu «. de ce Micromètre avec la longueur d'environ onze pieds « onze pouces du foyer de l'objectif. Nous avions difpofé la « lunette parallèlement au rayon, en l'ajuftant fur un objet éloi- « gné, auquel on vifoit par le limbe \& par le centre; nous « nous étions auff affurés que les foies du Micromètre étoient “ perpendiculaires au limbe; puifqu'elles convenoient avec un « 
130 MESURE DES TROIS PREMIERS

is fil-à-plomb fufpendu à une affez grande diftance, pendant s que l'inftrument étoit couché, \& le limbe mis de niveau. „Enfin nous avions marqué avec le plus grand foin, \& en „ prenant pour corde la dix-huitième partie du rayon, avec une " petite fraction que nous difcutâmes fcrupuleufement, un arc » de $3^{\mathrm{d}}$ I I' I $\frac{1}{5}$ ", vers le milieu duquel répondoit la lunette; \& " nous avions outre cela une Pendule déjà réglée par des hau„ teurs correfpondantes que chacun de nous avoit prifes, \& que »M. Verguin a enfuite principalement continué de prendre. "Ainf tout ce qui dépendoit de nous étoit entièrement dif". pofé, \& il ne nous manquoit plus qu'un ciel favorable pour »l'obfervation.

》Diverfes confidérations, qu'il eft inutile de rapporter ici, " nous avoient invité à nous fervir de l'étoile de la feconde " grandeur, qui eft au milieu de la ceinture d'Orion, \& que »Bayer a défignée par ع. Mais le temps, qui de jour ne nous " accordoit quà peine quelques hauteurs pour régler la Pen" dule, nons étoit encore plus contraire de nuit; \& pendant "plus d'un mois, nous n'avons fait autre chofe que recomoître " les changemens qu'il falloit faire à la direction de l'inftrument, \& " nous affurer enfuite, qu'il étoit exactement dans le plan du Mé" ridien. Nous remarquâmes auffi quelque défaut de folidité dans " le Micromètre, à quoi il nous fallut remédier; ce qui nous fit " perdre quelques obfervations dont nous étions contens.

„ Nous dirigeâmes l'inftument par le moyen d'une Méri„ dienne, tracée avec exactitude, \& indiquée par un affemblage " de cheveux, long de plus de dix-huit pieds, \& tendu, quand " nous l'avons voulu, d'un côte de l'obfervatoire à l'autre fur » deux petits crampons attachés aux murailles oppof́es : cette 


\section{DEGRES DU MÉRIDIEN:}

précaution nous a valu la facilité d'examiner chaque jour fi " le limbe, qui a environ 25 pouces de longueur, étoit exac- " tement parallèle à la Méridienne, en mefurant fcrupuleufe- “ ment la diftance de l'un à l'autre avec une échelle divifée en “ très-petites parties; \& nous avons pû, lorfque nous avons * tourné \& retourné l'inftrument, le remettre infailliblement « dans la même direction à moins d'une demi-minute près. “ J'avois auffr reconnu le 27 \& le 29 d'Octobre, en comparant « par la Trigonométrie fphérique, l'inftant auquel j’avois obfervé “ du côté de l'orjent des hauteurs de $\theta$ d'Antinoiis \& de \& " d'Orion, avec l'inftant que ces étoiles paflèrent par le fil ver- “ tical de la lunette, que ces paffages fe firent au temps même « de la médiation; \& M. de la Condamine trouva la même chole « par des hauteurs correfpondantes, quil réuffit à obtenir la “ nuit du I o au I I Novembre.

L'étoile que nous avions choifie étant éloignée du zénith “ de notre obfervatoire de Tarqui vers le Septentrion, d'envi- * ron I degré $40 \frac{1}{2}$ minŭtes, notre manière d'opérer devant nous “ fournir immédiatement le double de cette diftance, l'arc de a $3^{\text {d I I }}$ I I I I $^{\prime \prime}$ fe trouvoit trop petit. Le Micromètre nous a fourni “ le furplus de chaque côté pendant que nous avons fait tomber “ fucceffivement le fil-à-plomb fur les deux termes de l'arc. “ Voici ces excès tels que nous les avons obtenus, $M$. de la “ Condamine \& moi: nous étions convenus d'obferver alterna- “ tivement, \& nous avons cependant prefque toûjours eu le “ loifir de regarder l'un \& l'autre dans la lunette à chaque ob- “ fervation. 2 
132 MESURE DES TROIS PREMIERS

Premieres obfervations de la difance de l'étoile $\varepsilon$ d'Orion ar zénith de l'obfervatoire de Tarqui, faites par le moyen 'un arc de $3^{d} I I^{\prime} I^{\prime \prime} \frac{x}{5}$.

Le limbe de l'inftrument étant tourné vers l'orient.

Le 12 Novembre $1739 \ldots . . .1055$ parties microms. additives.

Le 13 .Nov. ........... 1054

Le limbe étant tourné vers l'occident, \& l'inftrument calé Sur l'autre tèrme de l'arc.

Le Is Novenbre. . . . . . . 318 parties additives.

Le 19 Nov............... 314

Le 27 Nov. . . . . . . . 318

“Selon ces obfervations, l'arc marqué fur le limbe étoî̊ „ trop petit d'un peu plus de I 37 I parties du Micromètre, s qui valent I $\mathrm{O}^{\prime}$ I"; \& par conféquent la diftance "apparente » de l'étoile $\varepsilon$ d'Orion au zénith, étoit au mois de Novembre „ dernier, de $\mathrm{I}^{\mathrm{d}} 4 \mathrm{O}^{\prime} 3 \mathrm{I}^{\prime \prime}$.

\Cette détermination étant achevée, nous voulûmes pouffer » la certitude plus loin, \& que les quantités fournies par le „ Micromètre devinflent fouftractives, d'additives qu'elles étoient, » ou que la Iunette fût pointée au deflous de l'étoile, au lieus s d'être pointée en deffus; afun que les nouvelles obfervations „ fuffent abfolument indépendantes des premières. Nous mar" quâmes pour cela fur le limbe un intervalle, dont la corde » étant exactement la dix-feptième partie du rayon, étoit de $\Rightarrow 3^{d} 22^{\prime}$ I $5^{\prime \prime}$. Cet arc étant trop grand, \& les quantités que " devoit fournir le Micromètre, négatives, les petites erreurs, 2. s'il y en avoit, \& qui pouvoient venir, ou du Micromètre, » ou de la difpofition. de la lunette, devoient néceffairement s fe trouver en fens contraires, \& fe manifefter par conféquent s mieux. Nous craignions, en opérant trop fervilement de las 
mème manière, de faire naître dans nos réfultats une confor- a mité qui nous trompât; au lieu que nous n’étions pas fâchés, “ \& nous nous le propofions même, d'y apercevoir, s'il le « falloit, des différences qui pûfeent nous inftruire.

Secondes obfervations, faites par le moyen d'un arc

$$
\text { de } 3^{d} 22^{\prime} I 5^{\prime \prime} \text {. }
$$

Le limbe de l'inftrument étant tourné vers l'occident.

Le 8 Décembre $1739 \ldots \ldots 65 \frac{5}{2}$ parties microm. négatives.

Le $9 \ldots \ldots \ldots 65 \frac{5}{2}$

Le $12 \ldots \ldots \ldots 64 \frac{1}{2}$

Le limbe étant tourné vers l'orient, \& l'inftrument calé fur l'autre extrémité de l'arc.

Le ${ }_{3}$ Décembre matin ..... 72 parties microm. négatives。

Le 13 Décembre foir. . . . . . 72

$$
\text { Le limbe retoumé yers l'occident. }
$$

Le 14 Décembre. . . . . . 70 parties microm. négatives.

Ces fecondes obfervations nous apprennent que le nouvel « arc étoit trop grand de 138 parties du Micromètre, qui va- " lent $I^{\prime} \frac{I}{2}$, \& que la diftance de l'étoile au zénith eft de $I^{d}$ * $40^{\prime} 37^{\prime \prime}$; \& fi on prend le milieu entre les deux réfultats pré- “ cédens, il vient $\mathrm{r}^{\mathrm{d}} 4 \mathrm{O}^{\prime} 34^{\prime \prime}$ pour la diftance apparente dont “ il s'agit.

Mais cette différence de 6 fecondes, qui fe trouve dans “ nos deux conclufions, \& dont une partie doit être attribuée à " des erreurs qui ne font erteurs que parce que nous n'en favons pas " préeifément la caufe, peut venir auffi un peu de quelque paral- " laxe que nous avons remarqué dans les fils du Micromètre; “ \& qui nous avoit obligé de mettre un diaphragnie proche de l'cil. “ Quoique l'incertitude que caufât ce défaut, torfqu'on prend. a. 


\section{I3 MESURE DES TROIS PREMIERS}

"le milieu entre les deux déterminations, ne fût que de 3 ", " \& qu'il n'en tombât que le tiers (une feconde) fur la gran„ deur du degré terreftre, nous avons cru que nous ne devions " pas, dans une circonftance fr importante, négliger de le cor» riger, puifque nous le comnoiffions. Le quinze Décembre, en " touchant feulement au porte-objectif, jaccourcis la lunette > d'environ une ligne; \& l'obfervation faite le lendemain miayant » appris, par une moindre parallaxe qu'avoient encore les fils, "mais en fens contraire, que javois produit un trop grand »-accourciffement, je travaillai le $\mathbf{~} 7$ à en détruire une partie: la " lunette, de cette forte, n'a pas été racourcie d'une ligne, \& " ce changement n'a pî en apporter aucun de fenfible dans la " valeur des parties du Micromètre, comme il eft facile de s s'en convaincre, fur-tout à l'égard de la petite quantité que " nous avions à mefurer. Mais foit qu'en touchant à la lunette, » je lui eufle donné quelque facilité à fe déranger, ou foit que "l'inftrument eût reçû quelque coup entre les obfervations, "nous avons enfuite eu le chagrin de ne pas profiter de plu"freurs belles nuits quili a fait pendant le refte du mois de "Décembre. Il feroit inutile de faire ici le détail de tous les " accidens qui nous font arrivés. C'eft principalement dans cette » rencontre que nous avons éprouvé combien étoit prudente » la réfolution que nous avions prife dès les commencemens, ¿ de faire des obfervations indépendantes les unes des autres, " pour voir fi elles donnoient le même réfultat; de changer " même l'état de l'inftrument, \& de le tourner plufieurs fois " dans le cours des obfervations. Cette attention, qui devient " plus néceffaire à mefure que l'inftrument eft plus grand, \& " qu'il eft formé d'un plus grand nombre de pièces, devoit 
mous apprendre fi le nôtre, qui a une brifure au milieu du “ rayon, \& dont les deux parties font jointes par plufieurs vis “ \& plufieurs clavettes, fouffroit quelque dérangement, malgré “ ce que nous avions fait pour le rendre folide, \& les précau- \& tions prefque fuperftitieufes avec lefquelles nous le touchions. « Enfin après l'avoir examiné une dernière fois depuis le haut “ jufqu'au bas le 29 Décembre, \& nos foupçons ne pouvant “ tomber que fur la lunette, quoiqu'elle füt arrêtée en trois en- “ droits, nous lui fimes diverfes ligatures avec du fil de fer affez « fort; ce qui nous a valu les obfervations fuivantes.

Troifiemes obfervations, faites par le moyen de lars

$$
\text { de } 3^{d} 22^{\prime} I 5^{\prime \prime}
$$

Le limbe vers l'occident.

Le 30 Décembre $1739 \ldots \ldots 75 \frac{1}{2}$ parties microm. négatives.

Le limbe tourné vers l'orient.

Le 2 Janvier $1740 \ldots \ldots . . .83$ parties micron. négatives.

Le limbe retourné vers l'occident.

Le 6 Janvier $1740 \ldots \ldots . \ldots 60$ parties négatives.

Le 9 Janv.. . . . . . . . . . 62

Il fuit de ces obfervations, que l'arc de $3^{\mathrm{d}} 22^{\prime}$ I $5^{\prime \prime}$ eft et trop grand de I 49 parties, qui valent $I^{\prime} \varsigma^{\prime \prime}$; \& Ia diftance « de l'étoile au zénith eft donc de ${ }^{\mathrm{d}} 4 \mathrm{O}^{\prime} 35^{\prime \prime}$. Cette troifième “ détermination tient le milieu entre les deux, I $^{\mathrm{d}} 4 \mathrm{O}^{\prime} 3^{\prime \prime}$ \& a I $40^{\prime} 37^{\prime \prime}$, que nous avions déjà trouvées en même temps « qu'elle eft plus voifme de la feconde : auffr regardions-nous les « deux premières comme deux épèces de limites; nous ne a doutions pas que fi l'une péchoit en excès, l'autre ne le fit en defaut, \& nous devions, outre cela, ạioûter plus dẹ foi os 


\section{I36 MESURE DES TROIS PREMIERS}

» à la feconde, puifque le Micromètre n'elt jamais plus exact " que lorfqu'il mefure de plus petites quantités. Enfin on peut " maintenant, fans que cela apporte guère plus d'une demi-fe" conde de différence fur la grandeur du degré terreftre, prendre " le milieu entre les trois réfultats, ou le prendre.feulement entre " les deux dernièrs. Ce fecond parti nous paroiffant préférable, "nous nous arrêtons à I ${ }^{\mathrm{d}} 4 \mathrm{O}^{\prime} 36^{\prime \prime}$ pour la diftance apparente dont "l'étoile \& d'Orion eft éloignée du zénith de notre obfervatoire de

"Tarqui, du côté du Septentrion, à la fin de I 73.9; \& ajoûtant

" une feconde pour la réfraction, il nous vient I ${ }^{\mathrm{d}} 4 \mathrm{O}^{\prime} 37^{\prime \prime}$ pour

" la diftance vraie. FA I T à Tarqui, le dix Janvier mil fept cens quarante. Signé BOU GU ER»; \& enfuite eft écrit:

" Je certifie la vérité des faits contenus dans le rapport pré" cédent. Je n'ai pû affifter à quelques-unes des opérations pré» paratoires, dont il eft fait mention au commencement de » cet écrit; mais j’en ai eu connoiflance dans le temps, \& de " plus, $\ddot{j}$ ai remefuré le 8 Octobre dernier la valeur du premier " arc, tracé en mon abfence, de $3^{\mathrm{d}} \mathbf{I}_{\mathbf{I}} \mathbf{I}^{\prime}$, en comparant la " corde à la lorgueur du rayon, \& nos mefures fe font accor" dées dans la demi-feconde. Nous répétâmes encore cette véri„ fication enfemble avant que de marquer le nouvel arc, dix" feptième partie du rayon, dont nous nous fommes fervis dans " nos deux dernières Suites d'obfervations. La nuit du I o au I I „Novembre, j’eus plufreurs hauteurs correfpondantes de l'étoile ๖ $\varepsilon$ d'Orion, qui nous affurèrent qu'elle paffoit par le fil vertical " de la lunette à une feconde près de l'heure viaie de fia média"tion : le refte a été fait enfemble, \& de concert, à Tarqui, "le onze Janvier I 740 . La fanté de M. Bouguer l'ayant obligé " de prefler fon départ, \& nos obfervations étant ainfi terminées, 
DEGRÉS DU MERIDIEN.

jai fouhaité d'avoir, avant que de démonter l'infurument, en- ce core une diftance de l'étoile $\varepsilon$ d'Orion au zénith, en retoumant « le limbe une feconde fois du côté de l'orient, pour fervir de c confrrmation à celle du 2 Janvier, la feule que nous avions dans ex cette fituation du limbe pendant le cours de nos troifièmes obfer- a vations. Le I 3 Janvier au foir, aidé de M. Verguin , jobfervai “ la diftance au zénith de la même étoile $\varepsilon$, de 95 parties du Mi- “ cromètre négatives, au lieu de 83 que nous avions trouvées le 2 “ Janvier. En prenant le milieu de cette dernière obfervation, « dont jai été content, \& de celle du 2 Janv. dont nous le fûmes “ aufi, \& qui diffèrent entr'elles de cinq fecondes, la diftance de «. l'étoile au zénith , tirée de nos troifièmes obfervations, fe trou- « vera diminuée d'une feconde un quart. Cette diftance fera, à un « quart de feconde près, moyenne entre celle qui réfulte de nos « premières \& fecondes obfervations, \& elle ne changera le réful- « tat ci-deffus adopté, que d'environ une demi-feconde. Avant « que de démonter l'inftrument, nous avons repris, M. Verguin ce $\&$ moi, la mefure du rayon, \& celle de la corde de l'arc, que « nous avions trouvée être exactement la dix-feptième partie du a rayon : cette corde, prife avec l'ouverture d'un compas à arc « à plufieurs reprifes, \& portée dix - fept fois d'un bout à l'autre c du rayon, excédoit à la dernière fois le rayon de $\frac{28}{100}$ de ligne, “ ou les $\frac{4}{5}$ d'une partie égale de mon compas de proportion. FAIT a à Tarquit; le I 5 J.er matin I 740 . Signé LA CON DAMINE; ce \&. plus bas eft encore écrit:

Je foufligné, certifie la vérité de tous les faits rapportés ci- ec đeflus, tant pour avoir affinté à tout, que pour y avoir aidé. J'ai « auffi eu le loifr de regarder à la lunette dans prefque toutes “ les obfervations, \& j’ai vû l'étoile fuivre le fil du Micromètre. « FA I T à Tarqui, te I 5 J.er I 74 o. Signé VERGUIN.». 


\section{A R T I C L E VII. \\ Table d'obfervations de l'E'toile \& d'Orion,}

Faites en commun à Tarqui en 1739 , réduites au premier Janvier 1743.

Première Suite d'obfervations, faites par le moyen d'un Arc de $3^{\mathrm{d}} \mathrm{I}^{\prime} \mathbf{1}^{\prime \prime}, 2$, dont la corde étoit de $4^{\prime \prime}$ plus grande que la $18^{e}$ partie du rayon.

\begin{tabular}{|c|c|c|c|c|c|c|c|c|}
\hline $\begin{array}{c}\text { Situation } \\
\text { du } \\
\text { Secteur. }\end{array}$ & $\begin{array}{c}\text { D A T E } \\
\text { des } \\
\text { Obfervations. }\end{array}$ & $\begin{array}{l}\text { QuAN TITE'S } \\
\text { obfervées avec } \\
\text { le Micromètre }\end{array}$ & $\begin{array}{c}\text { E'QUA } \\
\text { Précerfion } \\
\text { des } \\
\text { E'quinoxes }\end{array}$ & \begin{tabular}{|c|} 
Aberration \\
de ha \\
Lumiere.
\end{tabular} & $\begin{array}{l}\text { Nutation } \\
\text { de l'Axe } \\
\text { terreltre. }\end{array}$ & $\begin{array}{c}\text { RE'DUCTION } \\
\text { aU I Janvier } \\
\text { ' } 743 .\end{array}$ & $\begin{array}{c}\text { Quantite's } \\
\text { moyennes. }\end{array}$ & L T A T. \\
\hline $\begin{array}{l}\text { Le limbe } \\
\text { tourné } \\
\text { à l'orient. } \\
\text { àloccident. }\end{array}$ & $\begin{array}{l}12 \mathrm{~N} \\
13\end{array}$ & 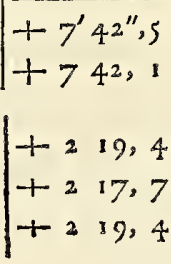 & $\begin{array}{r}+9,9 \\
+9.9 \\
+9.8\end{array}$ & $\begin{array}{l}\left|\begin{array}{l}-6,9 \\
-6,8\end{array}\right| \\
\left|\begin{array}{l}6,6 \\
-6,1 \\
-5,1\end{array}\right|\end{array}$ & $\begin{array}{l}+8,7 \\
+8,7 \\
+8.7\end{array}$ & 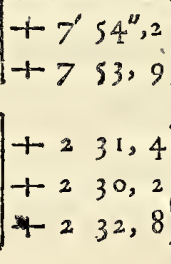 & 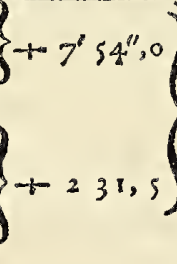 & 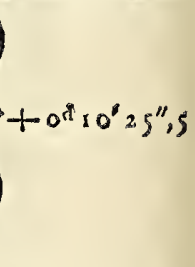 \\
\hline
\end{tabular}

Arc du Secteur. ................... 3 1 1 1, 2

Double diftance obfervée au Zénith $\ldots \ldots 3^{\mathrm{d}} 21^{\prime} 26^{\prime \prime}, 7$

Premicr Réfultat. Diftance apparente de $€$ d'Orion au Zénith de Tarqui du côté du Nord,

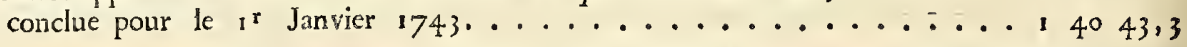

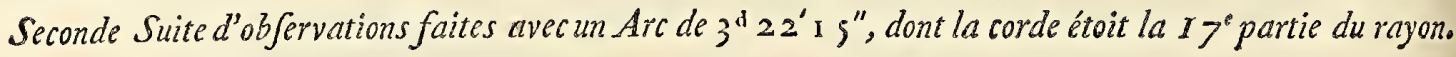

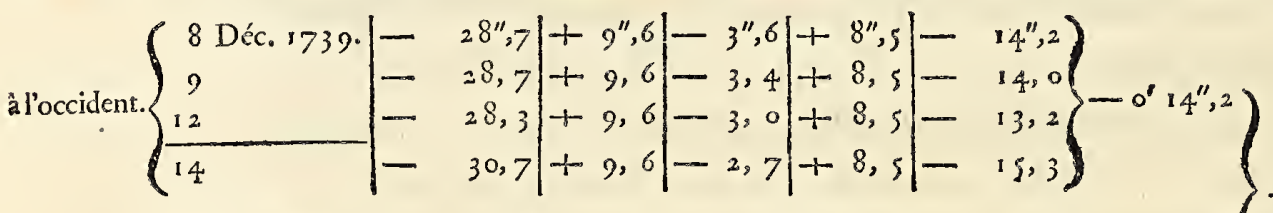

2 lorient. $\left.\left\{\begin{array}{ll}13 & \text { matin: } \\ 13 & \text { foir. }\end{array} \mid-\begin{array}{l}31,6|+9,6|-2,9|+8,5|-16,4 \\ 31,6|+9,6|-3,9|+8,5|-16,4\end{array}\right\}-16,4\right\}$

Arc du Secteur.................... 22215

Double diftance obfervée au Zénith ..... 3 Second Réfultat. Diftance apparente de $\varepsilon$ d'Orion au Zénith de Tarqui du côté du Nord,

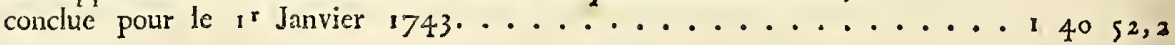

Troifieme Suite d'objervations avec le même Arc, après avoir changé la fituation de l'Objectifo

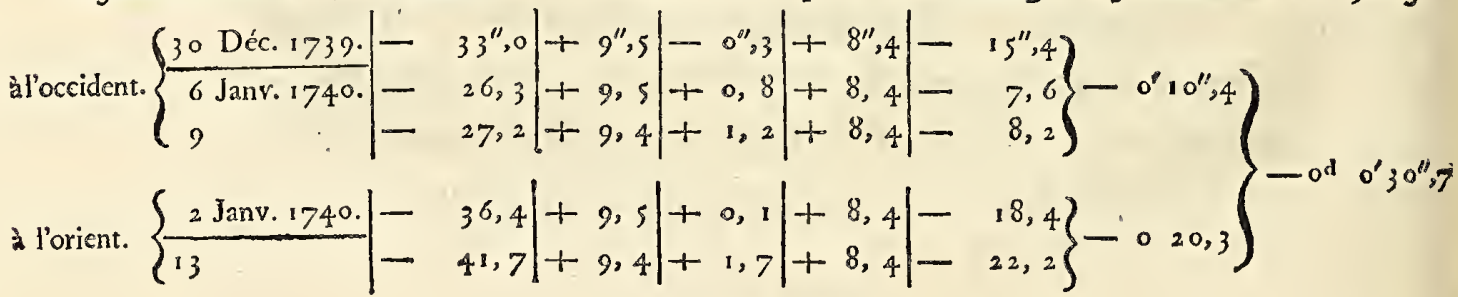

Arc du Secteur ....................... 222 is

Double diftance obfervéc au Zénith .....

Troifieme Réfuliat. Difance apparente de \& d'Orion au Zénith de Tarqui du côté du Nord,

conclue pour le $x^{x}$ Janvier $7443 \ldots \ldots$................... 42 , 
Remarques fur les obfervations de la Table précédente.

Dans la Table qui précède, chaque trait horizontal qui coupe la colonne des dates des obfervations, fert à indiquer que le Secteur a été retouné dans l'intervalle des deux obfervations féparées par le trait. Le refte de la Table parte affez aux yeux, \& n'a pas befoin d'explication.

On voit par cette Table, que les obfervations faites en I 739 à Tarqui, étant réduites à l'époque du premier Janvier 1743 , le premier des trois réfultats diffère des deux fuivans de 9"; au lieu que la différence n'eft réputée que de 6" dans le procès verbal, qui a été drefié dans un temps où Ies loix de l'aberration de Ia lumière nous étoient inconnues.

On voit auffi que le fecond \& le troifième réfultats, qui paroiffoient, fuivant le même procès verbal, différer entr'eux de deux ou de trois fecondes, felon qu'on tenoit compte ou non de l'obfervation du 13 Janvier I 740, s'accordent à moins d'une feconde, depuis que les équations ont été appliquées.

En général, les équations employées pour la réduction de ces obfervations, au premier Janvier 1743 , les rapprochent de quelques fecondes du réfultat de nos obfervations fimultanées, à l'époque defquelles je les ai réduites. Mais la diftance de l'étoile au zénith, tirée de celles que j'examine ici, eft, toute déduction faite, encore trop grande de 27 à 28 fecondes par le premier réfultat, \& de 18 par le fecond; en forte que l'erreur moyenne eft de $22^{\prime \prime} \frac{1}{2}$ au moins.

L'état d'imperfection où étoit alors notre Secteur; fa facilité à fe déranger quand on le retournoit, de quoi nous nous fommes plufieurs fois convaincus depuis; les diver's défauts 
que nous y remarquâmes dès-lors, \& que j'examinerai plus en détail; la différence de 9 fecondes entre le premier réfultat \& les deux derniers; celle de 7 fecondes entre l'obfervation du 3 o Décembre 1739 \& celle du 6 Janvier 1740 , employées dans le demier réfultat: enfin, \& plus que tout le refte, la diftance de la même étoile au zénith, trouvée conftamment de 20 \& tant de fecondes plus grande par M. Bouguer pendant fept à huit mois en I 74I, en obfervant fur différens arcs; \& par moi pendant autant de temps en I 742 \& I 743 , avec un nouvel arc \& un nouveau rayon, \& en prenant les nouvelles précautions qui feront expliquées; tout cela eft plus que fuffifant pour nous affurer que l'erreur eft certainement dans les obfervations de I 739. Mais il refte à en démêler 1 a fource. Les erreurs, dont il eft permis d'ignorer la caufe. doivent varier en plus \& en moins: fi celle dont il s'agit ici étoit de cette efpèce, il n'y a nulle vrai-femblance que cette erreur eût été conftante pendant le cours de plufreurs mois; \& que trois différentes Suites d'obfervations, indépendantes l'une de l'autre, faites fur différens arcs avec une lunette dont l'objectif a été changé de place, \& avec un inftument tourné \& retourné plufieurs fois en fens contraire, fe fuffent accordées à donner une diftance au zénith inégale à la vérité, mais toûjours confidérablement moindre qu'elle ne parût en 1739 . Le hafard, que je prends ici pour une combinaifon inconnue de caufes variables, n'admet point une fi grande uniformité; ou, pour parler plus exactement, ce feroit-là un cas unique entre une infinité de cas très-peu vrai-femblables. Cependant, puifqui il feroit poffible, on pourroit le fuppofer réel, s'il ne s'agiffoit que d'une très-petite quantité; mais 20 fecondes \& plus en font. 
une trop confidérable, pour n'être que la fomme de ces petites erreurs dont les obfervateurs les plus attentifs ne peuvent quelquefois fe garantir. C'eft ce qui m'a engagé à examiner fcrupuleufement les différentes caufes qui ont pû nuire à Ia jufteffe de ces obfervations, \& à évaluer les effets de ces caufes. Je parle ici de celles qui ne fe prélentent pas au premier coup d'œil, \& auxquelles ont fait ordinairement peu d'attention. Je vais rendre compte de cet examen, que je terminerai en propolant ce que je crois le plus vraifemblable \& le plus propre à expliquer pourquoi l'erreur de nos premières obfervations en 1739 , nous avoit fait conftamment trouver la diftance de l'étoile au zénith, plus petite que la véritable.

\section{A R T I C L E VIII.}

'Examen des différentés caufes qui peuvent mire à la juftefe des obfervations.

Des effets du froid o du chaud sur notre Secteur.

J'A I remarqué dans la defcription du Secteur (art. II), que fon limbe étoit formé d'une règle de cuivre, attachée avec des clous de même métal, rivés fur une bande de fer, laquelle étoit foûtenue d'une règle de chan pareillement de fer; ainf, quoique ta matière propre du limbe funt plus fufceptible de dilatation \& de condenfation que la bande de fer qui lui fervoit d'appui, ce dernier métal réfintant à l'effort du cuivre, ne lui permettoit pas de fe dilater, ni de fe contracter plus que le fer même. On peut donc regarder notre Secteur, dont 


\section{I42 MESURE DES TROIS PREMIERS}

le limbe ne faifoit qu'une très-petite partie, comme s'il étoit tout d'une même matière: or on voit que dans ce cas, toutes fes parties fe dilateroient \& fe condenferoient proportionnellement; \& par conféquent, qu’il ne changeroit pas de figure, par les alternatives du chaud \& du froid.

Mais quand on fuppoferoit que le limbe de cuivre auroit eu la liberté de prendre toute l'extenfion que la chaleur pouvoit lui donner, il n'en réfulteroit encore qu'une très-petite variation dans l'arc qui a fervi aux obfervations : car le rayon \& les autres parties de l'inftument, qui font de fer, s'alongeant en même temps que le cuivre, \& par la même caufe, le changement de figure qui furviendroit à l'inftrument, ne feroit cauré que par l'excès de la dilatation du cuivre qui forme le limbe, fur celle du fer qui lui eft adoffé.

J’ai trouvé par les expériences dont j’ai déjà parlé, que l'alongement du fer qui répondoit à une différence de dix degrés de chaleur, indiquée par le Thermomètre. de $M$. de Reaumur (c'elt-à-dire, à une augmentation de la centiène partie du volume quioccupe la liqueur lors de Ja congélation ), étoit de o, ligne o r 2 fur la longueur d'une toife.

Si, d'après ces expériences, $\&$ en fuppofant que le rapport de la dilatation du fer à celle du cuivre eft comme 8 à I I , on prend la peine de calculer de combien la partie de notre limbe de cuivre, qui répondoit à l'arc de I degré $\frac{2}{3}$, a dû s'alonger plus que le fer, \& quelle différence cet alongement a dû produire fur l'amplitude de cet arc; on trouvera que la différence répond à peine à une demi-feconde pour dix degrés de variation dans le Thermomètre : ce qui, comme on voit, n'eft. qu'une quantité imperceptible. 
Que fera-ce fi l'on fait attention que dans les lieux frais \& bas, où nous avons toûjours fait nos obfervations, \& dont nous avions foin d'interdire l'accès à l'air extérieur, le Thermomètre varioit à peine du tiers de la quantité fuppofée? On voit bien que quand même on admettroit un plus grand rapport entre les dilatations du fer \& du cuivre, les variations n’iroient jamais qu'à une petite fraction de feconde; \& par conféquent il eft démontré que l'action du froid \& du chaud fur notre Secteur n'a pû caufer que des changemens fort au deflous de ceux que les bornes de nos fens nous permettent d'apercevoir.

Cette température toûjours à peu près égale de nos obfervatoires, \& les précautions que nous avons toûjours eues de ne découvrir l'ouverture du toit, qui répondoit à la lunette, qu'au moment de l'obfervation, doivent autant nous raffurer fur la crainte du relẩchement des foies pofées au foyer du Micromètre, que fur les effets de la dilatation du limbe par la chaleur. D'ailleurs, la fituation du Secteur approchoit fi fort de la verticale, que la projection de la courbure, produite par le poids des fils relâchés, ne pouvoit jamais différer fenfiblement de la ligne droite.

\section{A R T I C L E IX.}

Suite de lexamen des différentes caufes \& $c$. De la flexion de l'Inftrument dans le plan du Limbe.

JE confonds ici la flexion de l'inftument avec celle de la lunette, \& elles ne doivent pas être diftinguées dans notie 


\section{MESURE DES TROIS PREMIERS}

Secteur, non plus que dans celui de M. Graham, qui a fervi aux obfervations du Nord. Quelque différent que fût le nôtre dinns fa conftruction, de celui-ci, ils avoient tous deux cela de commun, que la lunette, dans l'un \& dans l'autre, étoit précifément de la même longueur que le rayon de l'inftrument, \& pouvoit être prife pour le rayon même. Celle de notre Secteur étant, comme je l'ai expliqué (article 11), appliquée le long de la barre de fer qui formoit le rayon, participoit à tous fes mouvemens. C'eft du moins ce que je fuppofe quant à préfent ; \& il ne s'agit dans cet article que d'examiner combien ta barre de fer, qui formoit le rayon du Secteur, lequel étoit adoffé à la tunette, \& que je ne dítingue pas ici de la lunette même, a dû fléchir \& fe courber par fon propre poids pendant nos obfervations, \& de combien cette courbure a pû changer l'amplitude de l'arc obfervé fur le limbe.

Je cherche d'abord fi l'erreur qu'a pû caufer cette flexion a dû augmenter ou diminuer la diftance apparente de l'étoile au zénith. II s'agit de la flexion dans lé plan du limbe, ou dans le plan du Méridien, ce qui revient ici au même.

Planche I, Il faut remarquer que de la manière dont notre inftruGg. 8 . ment étoit fufpendu, fes deux extrémités étoient appuyces, l'une fur le colier $K$ de fufpenfion, l'autre fur l'une des vis de régie $n, n ; \&$ par conféquent la convexité de la courbure devoit être en deffous, au contraire de ce qui feroit arrivé $f_{i}$ le Secteur en̂t été fufpendu par fon centre de gravité, vers le milieu de la longueur de fon rayon, comme lors de notre obfervation de l'obliquité de l'Écliptique en 1736 \& 1737. On voit bien que les efiets de la courbure doivent être totalement oppofés dans ces deux cas. 
La Figure repréfente le rayon vifuel dirigé à l'aftre dont on obferve la diftance au zénith; \& pour éviter la confufion, I'on n'y a pas diftingué la lunette du rayon $C A$, fur lequel elle eft appliquée. Si ce rayon vient à fléchir par fon propre poids, fuivant la courbe $C E A$; le limbe, ou pluftôt la ligne $A B$ qui le repréfente ici, \& qui fait un angle droit avec le rayon $C A$, deviendra dautant plus oblique à l'égard du fil-à-plomb $C P$, que la courbure $C E A$ fera fuppofée plus grande. Cette même ligne fera, par exemple, tranfportée par la flexion du rayon, de $A B$ en $A b$. Le fil-à-plomb coupera donc le limbe du Secteur en $b$, à une plus grande diftance de $A$ que dans fa première fituation $A B$; \& par conféquent il marquera un plus grand nombre de degrés fur le limbe, quoique l'angle $A C B$, entre le rayon vifuel $A C \&$ le fil-ì-plomb $C b$, demeure le même.

Pour ne pas embrouiller la Figure, on a auff fuppofé que le point $A$ reftoit le même après la flexion du rayon. Quoique cette flexion doive le rapprocher du point $C$, c'eft d'une quantité qui ne fauroit mériter qu'on y fafle la moindre attention, puifqu'elle ne feroit que de $\frac{1}{850}$ de ligne, dans le cas où la flèche $D E$ de la courbure feroit d'une ligne entière.

La courbure du rayon, par la nature de la fufpenfion de notre Secteur, devoit donc augmenter la diftance apparente au zénith: ainf la flexibilité de l'inftrument, moins folide dans les premières obfervations qu'il ne l'a été depuis, n'a nullement contribué en 1739 à nous faire trouver la diftance de l'étoile au zénith de Tarqui moins grande que la véritable. C'eft une réflexion que je communiquai à M. Bouguer en lui écrivant de Tarqui à Quito en 1742, \& en lui propofant mes conjectures, fur le défaut de nos anciennes obfervations, 
Mais en quelque fens que fe trouvât lierreur caufée par la flexion du rayon, elle ne pouvoit jamais être d'une dangereufe conféquence, vû la conftruction particulière de notre Secteur, dans lequel la lunette étoit égale au rayon, \& l'objectif répondoit au centre.

Pour m'affurer qu’on pouvoit négliger cette erreur , \& avant que d'avoir tenté aucune expérience fur la flexion des barreg de métal, j’avois fait une fuppofition forcée \& hors de la vrai-femblance, \& cherché quelle feroit l'erreur dans ce cas. J'avois fuppofé qu'en écartant la lunette de la ligne verticale d'un degré \& demi, ce qui étoit l'inclinaifon moyenne qui convenoit à nos obfervations, la flexion du rayon, caufée par fon propre poids, lui feroit prendre une courbure $C E A$ telle que $E A$ fit un angle de ro minutes avec la fituation. primitive du rayon $C A$; ce qui donneroit $2 \frac{1}{2}$ lignes à la flèche $D E ; \&$ javois trouvé dans cette fuppofition, que la diftance apparente au zénith feroit augmentée de moins d'une demifeconde : réfultat très-conforme à ce que M. de Maupcrtuis* a remarqué fur ce fujet.

Je jugeois que la flexion du rayon étoit beaucoup moindre que je ne l'avois fuppofée; mais pour ne rien admettre gratuitement, j’ai, depuis ce temps-là, mefuré la courbure d'une barre de fer plate, pofée horizontalement, \& foûtenue par fes deux extrémités, en forte que fa longueur entre jes deux appuis étoit de douze pieds : j’ai fait enfuite couper cette barre ; \& jar. mefuré la courbure d'une portion longue de fix pieds que j’en avois retranchée. J'ai conclu des mefures actuelles, doublées par le renverfemient de la barre, que la flèche de l'arc de cette

* Figure de la Terre déterminée \&c. Difcours \& c. page 67. 


\section{DEGRÉS DÚ MERTDIEN.}

dernière courbure étoit environ la dix-feptième partie de l'autre Hèche; ce qui approche beaucoup du rapport des quatrièmes puiffances des longueurs que les flexions doivent fuivre; comme M. Daniel Bernoulli l'a déduit de plufreurs expériences ingénieufes, \& l'a démontré dans la Pièce qui a remporté le prix de l'A cadémie en r 743 . J'ai fait d'autres épreuves fur la même barre, en la pofant tantôt à plat fur fa plus grande largeur, tantôt de chan fur fa plus étroite dimenfion; \& jai trouvé que les flèches des différens arcs de courbure d'une même barre, pofée fur fa face la plus large ou la plus étroite, fuivoient aflez exactement la raifon inverfe des quarrés de ta dimenfion qui fe trouvoit pofée verticalement dans chaque expérience.

Les mefures de notre Secteur étant connues, on peut conclurre des expériences précédentes la courbure qua dû contracter fon rayon.

La barre qui formoit le rayon de notre Secteur ayant trois pouces de large fur douze pieds de long, il a réfuité de toutes mes expériences, à quelques variétés près, qui ne peuvent manquer de fe rencontrer dans le phyfique, que la flèche de ta courbure que la barre devoit prendre dans une pofition horizontale de chan, n'étoit que d'environ une ligne : mais en approchant cette barre de la fituation verticale, le poids qui caufe fa courbure agit par un levier plus court; \& ce levier fe raccourcit comme le finus del'angle d'inclinaifon avec la verticale. Donc en fuppofant que la courbure diminue dans le même rapport, celle du Secteur, dirigé à I $\frac{1}{2}$ degré du zénith, ne fera plus que la 38 e partie de celle qu'il avoit étant pofé horizontalement. La flèche de la courbure horizontale étoit de I ligne; elle ne fera plus que de $\frac{r}{38}$ de ligne, c'eft-à-dire, prefque la centième partie de 


\section{MESURE DES TROIS PREMIERS}

ce que je favois fuppofée, en domnant $2 \frac{1}{2}$ lignes de lorigueur à $D E$. L'erreur, qui eût été en ce cas d'une demi-feconde, \& par conféquent inperceptible, fera donc déformais cent fois trop petite pour être aperçûe. J'ai donc pû̉ négliger fans fcrupule dans le calcul précédent le poids de la lunette, ainfi que celui de la règle de chan adoffée au rayon du Secteur; \& cela quand on voudroit fuppofer Ia flexion du tout, double ou triple, ou même décuple de celle que prendroit la barre toute feule.

If s'enfuit de tout ceci, que ni la flexion du rayon provenante de fon propre poids, ni celle d'une lunette de même longueur, appliquée fur le rayon, \& faifant pour ainfi dire corps avec lui, n'ont pû caufer aucune erreur fenfible dans nos obfervations.

J'ai épargné au Lecteur le détail des calculs précédens, \& de plufieurs autres quil m’a fallu faire, à quoi il n'y a de mérite que le courage néceffaire pour en furmonter l'ennui.

\section{A R T I C L E X.}

Continuation du même Jujet.

De la flexion du rayon dans le plan perpendiculaire à celui de l'inftrument; ơ du parallélifme de la lunette à ce même plan.

Tou ce qui a été dit jufqu’ici de la flexion du rayon, ne regarde que celle qui peut fe faire dans le plan du Secteur.

Quant à la flexion dans le fens perpendiculairé à ce même plan, elle ne pourroit être d'une conféquence dangereufe, qu'autant qu'elle attéreroit la figure de l'inftrument, en changeant Ia pofition du centre à l'égard de l'arc; \& c'eft ce qui n’a pâ 
arriver dans notre Secteur: car fuppofant, contre toute vraifemblance, que la barre de fer $D C$, qui portoit la lunette, fe Planclie II.

fût courbée en avant ou en arrière, malgié la règle de chan $J J$ qui foûtenoit cette barre, la courbure, quelque confidérable qu'elle eût pû être, n'eût pas changé le vrai rayon du Secteur, c'eft-ì-dire, la diftance du centre $I$ à l'arc $\alpha \omega$, tracé fur le limbe; puifque cet arc a toûjours été décrit, l'inftrument étant déjà monté, \& fufpendu verticalement, dans la même fituation où il a été maintenu depuis, laquelle a été conftatée à chaque obfervation, en examinant fi le fil-à-plomb pendant librement du centre rafoit le limbe fans y toucher.

La pofition du centre à l'égard de l'arc ne pouvant varier; Ja lunette, parallèle ou non au plan du Secteur, confervera toûjours la même fituation à l'égard de ce plan, pourvâ qu'elle y foit fermement attachée. Mais ce n'eft point affez pour un Obfervateur exact d'être affuré que l'angle de fa lunette, avec le plan de fon Secteur, eft conftant, \& qu'il ne peut changer d'une obfervation à l'autre: il faut réduire cet angle à zéro, \& rendre la lunette exactement parallèle au plan du Secteur; ou tout au moins il faut pouvoir mefurer cet angle avec précifion, pour être en état de calculer de combien le défaut de parallédifme de la lunette, peut changer la diftance apparente de l'étoile au zénith.

La vérification au zénith par la demi-révolution du Secteur fur fon axe, eft une opération difficile \& peu ufitée. Elle n'a guère été employée jufqu'ici, quà reconnô̂tre de combien la lunette étoit écartée, dans le plan du Secteur, du commencement de la graduation; ce qui eft ordinairement le point le plus important à vérifier dans les obfervations. 


\section{ISO MESURE DES TROIS PREMIERS}

Mais ce moyen imaginé par M. Picard, peut fervir à reconnoître de combien la lunette s'écarte du plan du limbe, tout auffbien qu'il fert à vérifier, $f_{1}$, dans le plan du limbe, elle s'éloigne du commencement des divifions. Je fuppofe la Méridienne tracée, le limbe du Secteur bien arrêté dans le plan du Méridien, la Junette pointée à la hauteur où doit paffer l'étoile, l'inftrument bien calé. Tout étant ainfi difpofé, on attendra l'heure de la médiation qu'on aura calculée d'avance. Si la lunette fait un angle avec le plan du Secteur, \& par conféquent avec le Méridien, l'étoile paffera dans la lunette pluftôt ou plus tard que l'heure du calcul; pluftôt, fi la lunette eft pointée trop à l'orient; plus tard, fi elle incline vers l'occident. Suppofé que ce foit de quatre fecondes, onen pourra conclurre, dans le pays où nous éions, que l'angle de la lunette, avec le plan du Secteur, eft d'une minute. Cet angle fera phus ou moins grand, à proportion du nombre de fecondes dont le paffage de l'étoile dans ta lunette avancera ou retardera fur theure de la médiation.

Pour une plus grande fûreté, on retournera l'inftrument en fens contraire : l'étoile, comme on le voit, doit paffer au fil vertical de la lunette, dans une des fituations du Secteur, autant de fecondes avant l'heure de fa médiation, que de fecondes après, dans la fituation inverfe. S'il y avoit quelque différence, ce feroit une preuve d'erreur dans l'heure calculée, ou dans la direction du plan du Secteur. On a des moyens de vériffer l'une \& l'autre.

L'angle de la lunette, avec le plan dúlimbe, étant une fois connu, on aura tout ce quili faut pour calculer de combiein on a pû être trompé fur la diftance apparente de l'étoile au zénith, \& juger par-là fi l'erreur mérite confidération. En ce cas, il fera plus commode de corriger la déviation même 
de la lunette, en touchant à l'objectif, \& en l'approchant ou en l'éloignant du plan de l'inftrument, de la petite quantité néceflaire pour rendre l'axe optique de la lunette parallèle au plan du Secteur. Cette quantité, toûjours proportionnelle à la longueur du rayon, fera aifée à trouver. Dans notre Secteur de douze pieds'de rayon, elle étoit d'une ligne, pour 8 fecondes de différence dans l'heure de la médiation.

Si l'on avoit commencé à obferver, avant que d'avoir une Méridienne tracée, il feroit encore poflible de mefurer l'angle de l'axe de là lunette avec le limbe, pourvû quion eût l'heure de la médiation de l'étoile avec beaucoup de précifion; comme, par exemple, fi l'on s'en étoit afluré par plufieurs hauteurs correfpondantes de la même étoile. Voici en ce cas conme on pourroit y réuffir. On dirigeroit d'abord la lunette à l'antre, en forte qu'il paflầt au centre des fils à l'infant précis de la médiation : par-là on feroit fûr que l'axe optique de la lunette feroit exactement dans le plan du Méridien : enfuite deux fils-àplomb attachés aux deux extrémités de la lunette, pourroient fervir à rendre ce plan fenfible, \& à reconnoître s'il eft parallèle à celui du Secteur. Mais plus l'antre eeft voifin du zénith, moins cette méthode eft praticable.

Si notre Secteur n'eût pas été dirigé exactement dans le plan du Méridien lors de nos ob́ervations de I 739, ou fi l'étoile n'eût pas été obfervée à l'heure précife de la médiation, nous euffions trouvé, par cela feul, une fauffe diftance au zénith; mais on a vû dans le procès verbal ci-deflus, avec quel fcrupule le concours de ces deux circonftances a été obfervé. Leur omiffion n'a donc pû avoir aucune part au défaut de ces obfervations; \& je puis, par conféquent, me difpenfer d'évaluer la 


\section{IS2 MESURE DES TROIS PREMIERS}

quantité d'une erreur que nous n'avons point commife.

Les réflexions précédentes, \& d'autres du même genre, n’ont dû fe préfenter \& fe développer qu’à mefure que les circonftances, les difficultés, \& la répétition fréquente des obfervations, y ont donné lieu : ainfi il n'eft pas étomnant que nos premiers effais fe foient reffentis du peu d'expérience que nous avions tous alors dans une forte d'obfervation rare \& peu familière aux Aftronomes les plus exercés. Au refte, l'application nouvelle que je viens de faire, de la méthode de M. Picard pour vérifier l'inftrument au zénith par le renverfement, a dû fe préfenter tôt ou tard à ceux qui, comme nous, ont été dans le cas de faire un grand nombre d'obfervations de diftances d'étoiles au zénith. Ainfr quoique cet Aftronome, \& ceux qui ont obfervé dans les mêmes circonftances que nous, n'aient pas prefcrit explicitement la vérification du parallélifme de la lunette au plan du Secteur, Iaquelle fé déduit des mêmes principes que la vérification ordinaire; je me garderois bien d'en conclurre qu'ils ne l'ont pas employcee, \& qu'ils n'ont pris aucune précaution contre les erreurs de l'inclinaifon de la lunette fur le plan de l'inftrument.

\section{A R T I C L E X I.}

\section{Continuation du même fujet.}

De la caufe qui a pî augmenter la diffance apparente de l'étoile au zénith, à Tarcqui en 1739.

J'omers ici l'examen de plufieurs caufes d'erreur, différentes des précédentes, \& le détail des attentions que nous avons ẹues pour nous en garantir; parce qu'il en fera fait mention 
dans le procès verbal des obfervations faites à Cotchefqui en I 740 , \& dans l'article où je rendrai compte des nouvelles précautions que j’ai prifes en obfervant à Tarqui en I 7428 \& 743 . II fuffit de remarquer ici que toutes ces fources d'erreur, tant celles qui ont fait le fujet des articles précédens, que celles qui me reftent à examiner, étoient également propres à augmenter \& à diminuer la diftance apparente de l'étoile au zénith ; outre que les variations qu'elles pouvoient occafionner dans cette diftance n'auroient produit que quelques fecondes de plus ou de moins. On ne peut donc imputer à aucune de ces caufes le défaut de nos premières obfervations, par lefquelles la diftance de l'étoile au zénith de Tarqui nous parut conftanment de 19 à 27 fecondes plus petite en'I 739 qu'en $174 \mathrm{r}$, 1742 \& 1743 . Tâchons de développer le principe de cette erreur conftante dans le même fens : c'eft en partant de faits certains, que je vais effayer de remonter à fa fource.

Si jamais on a pû regarder une obfervation comme trèsexacte; c'eft, fans contredit, celle de la diftance de l'étoile $\varepsilon$ d'Orion all zénith de Tarqui, à laquelle nous nous fommes arrêtés, \& dont je vais bien-tôt rendre compte. Cette détermination, fruit de près de deux années de travail, eft conforme au réfultat de plufieurs Suites d'obfervations indépendantes l'une de l'autre, faites à diverfes reprifes pendant le cours de l'année I 74 I par M. Bonguer: je l'ai trouvée la même à la fin del'année fuivante, \& pendant plufieurs mois en 1743 , avec le Secteur nouvellement reconftruit, \& par un nouvel arc; tellement que la différence entre le réfultat de M. Bouguer \& le mien, eft à peine de deux fecondes, lorfqu'on a égard à toutes les équations connues. Nous avions, lui \& moi, redoublé de foins z 


\section{IS4 MESURE DES TROIS PREMIERS}

d'attentions \& de fcrupules dans ces dernières obfervations; \& Ia folidité de l'inftrument étoit alors à toute épreuve. Tant d'uniformité dans des circonftances fi différentes, \& un fi grand nombre de confrimations, ne laiffent plus lieu $\grave{a}$ aucun doute. Nous pouvons donc regarder la diftance de $\varepsilon$ d'Orion au zénith de Tarqui, oblervée en I 74 I, I 742 \& I 743, comme Ia véritable. Ceci pofé, voici comme je raifonne.

Planche I, Nous n'avons pû trouver en 1739 la diftance de cette même étoile au zénith, conftamment plus petite que la vraie, que parce que l'arc $A \omega$, tracé fur le limbe, \& terminé par le fil-à-plomb $C P$, cet arc, que nous prenions pour mefure de Ia diftance verticale obfervée, étoit plus petit que l'arc qui la mefuroit réellement. Or pour que cela foit arrivé, il faut néceffairement que le rayon vifuel $A c_{*}$, dirigé de l' nil à l'étoile, ait été tranfporté (par quelque caufe que ce puiffe être) de $A C$ en $A$ c. En ce cas, \& non autrement, l'angle apparent $A C P$, entre le rayon $C A$ du Secteur \& le fil-à-plomb $C P$, aura été plus petit que l'angle vrai entre le rayon vifuel $A c$, dinigé à l'étoile, \& Ia ligne verticale $c p$; alors quoique la vraie diftance de l'étoile au zénith fût mefurée par l'arc $A o$, on aura pris l'arc $A \omega$ pour fa mefure, \& par conféquent on aura jugé la diftance au zénith trop petite. Pour qu'on ait commis Ia même erreur dans les deux fituations de l'infrument, il faut que tout ce que je viens de fuppofer foit encore arrivé Iorfque le Secteur ayant été retourné en fens contraire, \& le point $A$ reftan au même lieu, le point $\alpha$ s'eft trouvé répondre au point $\omega$, \& réciproquement.

Jufqu’ici je n'ai tiré que des conféquences néceffaires \& évidentes; il me refte à chercher ce qui a pû tranfporter 
conftamment le rayon vifuel de $A C$ en $A c$ dans les deux fituations inverfes du Secteur.

En examinant (art. $I X \& X$ ) les effets de la flexion de notre inftrument en différens plans, je n'ai pas diftingué la Iunette du rayon, fur lequel elle étoit appliquée ; \& j’ai comparé à cet égard notre Secteur à celui de M. Graham, dans lequel il n'y a d'autre rayon que Ia lunette même qui porte le limbe; ce qui fait que la lunette \& le rayon peuvent rigoureufement y être pris l'un pour l'autre. Il eft vrai que nous avons pû fuppofer la même chofe de notre inftrument, à l'égard de nos dernières obfervations en 1741,1742 \& 1743 ; parce qualors les fourchettes de fer $\mathrm{ZZ}$ fervant de fupport à Ja lu- Planche III. nette, avoient été raccourcies de moitié au moins, parce quon en avoit multiplié le nombre, \& qu'enfin la lunette avoit été affermie fur le rayon avec de nouvelles précautions dont il fera parté plus bas. On a pú auffi confondre la lunette avec le rayon dans le nouveau Secteur de huit pieds de rayon, qui fervit à Cotchefqui en I 742 à M. Bouguer, vû l'attention exprefle qu'il apporta fur ce point, dont nous avions reconnu toute l'importance. Mais il faut avouer qu'il n'en étoit pas de même dans le temps de nos premières obfervations à Tarqui en 1739 : alors la lunette, dans une longueur de douze pieds, n'étoit foutenue que par trois fourchettes affez foibles, \& dont la diftance au corps de l'inftrument étoit de fept pouces; ce qui faifoit un long levier, à l'extrémité duquel le poids de la lunette pouvoit agir fenfiblement. Voyons quel a dû en être l'effet.

Ces fourchettes ou fupports étant perpendiculaires au plan du Secteur, c'étoit fur-tout dans le fens de leur direction que devoit s'exercer l'action du levier; \&, à cet égard, elle devoit 


\section{IS6 MESURE DES TROIS PREMIERS}

fe réduire à approcher la lunette du plan du Secteur, toûjours parallèlement à efle-même, \& plus ou moins près, felon que le poids de la lunette ent fait plier plus ou moins les fourchettes: comme deux régles paralléles s'approchent d'autant plus, que les traverfes qui les joignent deviennent plus obliques. Or on voit que ce mouvement de la lunette ne pouvoit rien changer à la diftance apparente de l'étoile au zénith, pourvî qu'il fe fit parallèlement au plan du Secteur; comme il ne nous eft pas permis d'en douter, puifque l'étoile paffoit en effet à l'heure calculée de fa médiation, dans les deux fituations inverfes de l'inftrument, ainfi que le procès verbal en fait foi.

Ce n'eft donc pas la flexion dans le plan perpendiculaire au limbe; \& c'eft feulement la flexion dans le plan du timbe, Planche I, qui a pû tranfporter le rayon vifuel de $A C$ en $A c$ : tranfport fig. 9 . quỉl eft néceflaire d’admettre pour expliquer comment la diftance au zénith a pû être trouvée conftamment trop petite. Voyons ce que les fupports trop longs de la lunette ont pû opérer en ce fens. Quion fuppofe que la fourchette fupérieure étoit plus foible que les autres: il fe fera fait néceffairement un pli au tuyau de la lunette vers le point $E$, à quoi le poids de la monture de l'objectif aura pû contribuer*. Cette fuppofition n'a rien de contraire à la vrai-femblance; $\&$ fi on l'admet, tout s'explicquera très-naturellement. $C \alpha \omega$ repréfente le plan du Secteur; $C$ eft le centre; a $\omega$ eft l'arc tracé fur le limbe; $C A$, le rayon fur lequel la lunette étoit appliquée, à 7 pouces de

* Cette monture étoit compofée d'un bout de tuyau de cuivre, de trois plaques de même métal, dont deux affez épaiffes, \& de quatre vis: le tout enté à l'extrémité d'un tube d'une fimple feuille de fer blanc, de deux pouces de dianietre. 
diftance, dans un plan parallèle au plan du Secteur \& perpendiculaire à celui de la Figure; ce qui empêche d'y pouvoir repréfenter les trois fupports qui répondoient aux points $I, 2,3$, du rayon $C A$. Le pli que nous fuppofons, qui fe fera fait à la lunette vers $E$ par Ia foibleffe du fupport 3, \& peut-être par le poids de la monture de l'objectif, aura tranporté l'extrémité de la lunette de $C$ en $c$ : la même chofe fera arrivée lorfque l'inftrument aura été retourné fur fon axe $C A, \&$ que le point $a \&$ le point $\omega$ auront mutueflement changé de place: le tout fạs que le centre $C$ du Secteur, d'où pend le fil-à-plomb, ait changé de fituation. Dans l'une \& dans l'autre pofition du Secteur, l'angle apparent $A C p$, compris entre le rayon $A C$ $\&$ le fil-à-plomb $C P$, qui battoit fur le limbe au point $\omega$ ou au point $\alpha$, aura été plus petit que l'angle véritable $A c p$, compris entre le rayon vifuel, tranfporté par la flexion de la lunette de $A C$ en $A c$, \& la verticale $c p$ parallèle à $C P$. Cette caufe agiffant confamment, \& tendant également dans les deux fituations de l'inftrument, à augmenter la diftance apparente de l'étoile au zénith, doit avoir produit une erreur conftante, \& du même fens: laquelle fe combinant avec d'autres petites erreurs variables, aura paru tantôt plus, tantôt moins grande; mais toûjours dans le fens où agiffoit la caufe principale \& dominante. Toute erreur femblable, \& procédant d'une courbure de la lunette par fon poids, ne peut être reconnue par le renverfement*: c'eft ce que $M$. de Maupcrtuis a déjà remarqué \& expliqué. Cette opération ne peut fervir qu’à vérifier la poftion d'une lunette fuppofée inflexible.

11 faut avouer que l'ćtoile que nous obfervions ne paffant:

* Figure de la Terre déterminée \&c. Difcours \&c. page 67 \& 68. 
I 8 MESURE DES TROIS PREMIERS

guère qu'à I $\frac{2}{3}$ degré du zénith à Tarqui, il n'eft pas aifé de concevoir que la lunette, dans une fituation qui différoit fı peu de la verticale, ait pû s'arquer par fon propre poids, en y ajoûtant même celui de la monture de lobjectif; mais j'avoue que je ne vois point d'autre manière d'expliquer le tranfport réel \& conftant du rayon vifuel, de $A C$ en $A c$. $M$. Bonguer l'aura peut-être fait plus heureufement. Quoi qu'il en foit, il eft certain que depuis que les fourchettes ont été raccourcies, que leur nombre a été augmenté, \& que la lunette a été affermie \& aflurée par plufieurs liens dans toute fa longueur fur le rayon du Secteur; la diftance au zénith a été trouvée conftamment plus grande qu'auparavarit, \& toûjours fenfiblement la même à chaque obfervation.

Au refte, comme une demi-ligne répondoit fur le limbe de notre Secteur à une minute de degré, il fuffit que le bout objectif de la lúnette ait varié d'un fixième de ligne, pour avoir produit' une erreur conftante de 20 fecondes dans la diftance au zénith.

\section{A R T I C L E XII.}

Premières obfervations faites à Cotchefqui, extrémité Septentrionale de la Méridienne; en Février, Mars \& Avril $174^{\circ}$.

A usst-tost que nous eûmes terminé notre travail à une extrémité de la Méridienne, nous nous hâtâmes de paffer à l'autre, pour mettre le moindre intervalle pofible entre nos obfervations aux deux bouts de l'arc mefuré. J'obfervois encore à Tarqui le I 3 Janvier I 740 ; \& dès le I 2 Février fuivant, te 


\section{DEGRÉS, DU MERIDIEN.}

Seçeur étoit prêt pour l'obfervation, à plus de quatre-vingts lieues de diftance; quoiqu'il eût fallu le porter à bras, par des chemins difficiles, \& que M. Bouguer eût été obligé de s'arrêter plufreurs jours à Quito, pour faire faire à cet inftrument plufreurs réparations qui avoient été jugées néceflaires. Les réponfes qu'il me fallut faire à mon arrivée en cette ville, aux lettres que je reçûs du Viceroi \& c. fur les affaires dont j'ai parlé, m'y retinrent deux ou trois jours après le départ de $\mathbf{M}$. Bouguti. Je l'allai joindre à frx lieues de Quito, fur la montagne de Mohanda, où nous étions convenus de terminer notre mefure du Méridien du côté du Nord. Je le trouvai tout établi, \& l'inftrument déjà monté à Cotchefqui, maifon de campagne où il s'étoit arrêté, \& qui eft frtuée à la vûe de notre première Bafe \& de nos premiers Triangles, avec lefquels il étoit aifé de lier notre nouvel obfervatoire. Je vérifiai le i 8 Ia grandeur de l'arc tracé par M. Bouguer. Les jours fuivans, nous commençâmes à obferver.

Au lieu de m'étendre fur ces obfervations, je ferai beaucoup mieux de tranfcrire ici le détail qu’en a domné M. Bouguer dans un procès verbal qu'il a rédigé à Cotche qui même, \& fait certifier \& légalifer à Quito par trois Notaires, comme le précédent l'avoit été à Cuenca par le Corps de Ville.

EXTRAIT du Procés verbal des Obfervations faites a Cotchefqui en $174^{\circ}$.

$J$ 'ai cru devoir fupprimer dans cet extrait quelques détails étrangers alux obfervations qui font la matière du procès verbal.

«.... On avoit (c'eft $M$. Bouguer qui parle) affez reconnu a Tarqui lorfqu'on démonta (l'ingtrument), que fes afiemblages ne * 
I6O MESURE DES TROIS PREMIERS

" pouvoient fouffir aucun changement $(a)$; \& que s'il y avoit " quelque dérangement à craindre, il ne pouvoit venir que de la „ lunetie, appliquée à une trop grande diftance du plan du „Secteur. Cependant je fis non feulement raccourcir les four" chettes qui foutenoient cette lunette, les réduifant à $3 \stackrel{1}{2}$ pouces, "d'environ 7 qu'elles avoient; mais je fis fortifier la brifure du " milieu du rayon du Secteur par deux nouvelles bandes de „fer, arrêtées chacune par plufieur's vis; \& je fis outre cela " Couder à Cotchefqui le Micromètre même à la lunette, après "avoir déjà mis de fortes ligatures de fil de fer. Il n'eft pas "befoin d'infifter fur toutes les autres précautions que j’ai prifes " enfuite avec le même foin que je l'avois fait à l'autre extré"mité, foit pour rendre la Jinette parallèle au plan de l'inf" trument, foit pour rendre les foies du Micromètre perpen„ diculaires au limbe, foit pour les mettre exactement au foyer " de l'objectif pour les objets céleftes, foit enfin pour tracer „ fur le limbe un arc dont la corde fût une partie aliquote „ exacte du rayon. Ayant reconnu dès le i 4 Février, par une "détermination un peu anticipée (b), mais qui s'eft trouvée " pluntôt confirmée que corrigée par le Triangle que nous avons "formé depuis, \& dont $M$. de la Condanine \& moi venons "actuellement de mefurer les angles, que notre obfervatoire " étoit à un peu moins de 425 toifes plus au Nord que le - Signal de Tanlagoa, je favois très-exactement notre latitude,

(a) Si nous l'avons cru alors, nous nous fommes bien défabufés depuis fur cet article.

(b) Par une autre détermination plus directe \& faite plus à loifir, nous trouvâmes depuis, chacun de notre côté, feulement 414 ou 4 I 5 toifes. Voyez Table I'des Triangles, Ti, XXXIII, Col. XI. 
\& que le double de la diftance de.l'étoile $\varepsilon$ d'Orion à notre « zénith, que les obfervations nous fourniroient immédiatement, « feroit d'environ $2^{\mathrm{d}} 52^{\prime}$ : c'eft pourquoi je me determinai à o donner pour corde à l'arc la $20^{\circ}$ partie du rayon; ce qui c rendoit cet arc de $2^{\mathrm{d}} \mathrm{S}^{\prime} \mathrm{I}^{\prime} 54 \frac{1}{4}^{\prime \prime}$, \& le Micromètre devoit fup- * pléer au refte. Pendant que toutes ces chofes fe faifoient, on " travailloit à obtenir une Méridienne dans notre obfervatoire “ en réglant une Pendule par des hauteurs correfpondantes, que “ M. Verguin s'étoit chargé de prendre, \& qu'il a auffi toûjours “ prifes depuis. Cette Méridienne, indiquée comme à Tarqui “ par un long affemblage de cheveux, fut vérifiée encore le i 7 “ Février par des hauteurs prifes le matin, defquelles je me fervis “ pour conftater l'état de la Pendule; \& tout étant difpofé le “ même jour..... Nous convinmmes, $M$. de la Condamine \& « moi, d'obferver alternativement. Voici nos obfervations. “

Premières obfervations de la diffance de l'étoile \&d'Orion au zénith de Cotchefqui, faites par le moyen d'inn arc de $2^{d} S I^{\prime} S 44^{\frac{1}{4}}$.

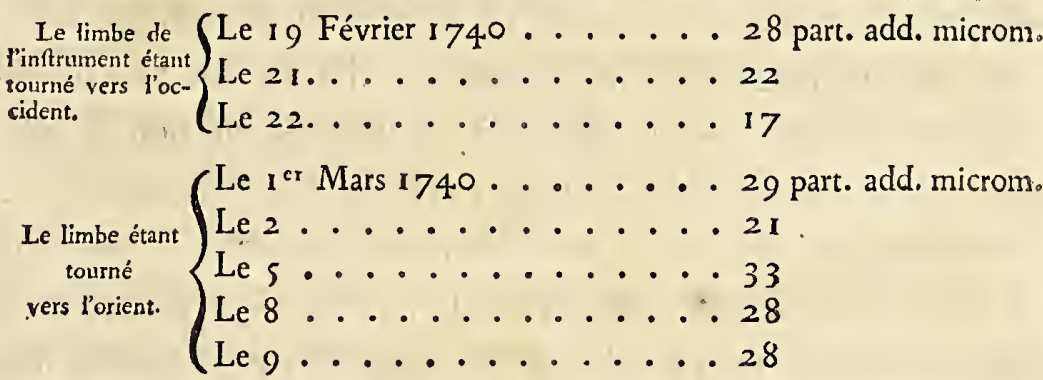

Nous retournâmes enfuite l'inftrument, en préfentant le ar limbe vers l'occident, dans l'intention de joindre quelques nou- “ velfes obfervations à celles des 19, 2 I \& 22 Février, \& de “ nous démontrer à nous - mêmes quiil n'y avoit eu aucun déran- os gement; mais un vent très-fort đqui s'éleva la nuit du 9 au o 
I62 MESURE DES TROIS PREMIERS

is I o Mars, \& qui continua jufqu'au I I matin, renverfa non " feulement le cuir qui fermoit l'ouverture du toit de l'obfer"vatoire, en rompant diverfes cordes, mais un fecond cuir qui " étoit beauçoup au deffous; \& qui étant appliqué fur un chaffis, " couvroit immédiatement l'inftrument : ce vent fit, pour ainfr " dire , pleuvoir les tuiles; nous en trouvâmes divers éclats tout " autour de l'inftrument, avec" des fragmens de chaux ou de " mortier; \& on ne pût pas empêcher le r o matin, pendant " que nous étions tous occupés à réparer ces défordres caufés " pendant la nuit, \& à tâcher de les prévenir pour une autre " fois, qu'une tuile entière ne tombât en notre préfence, non " pas fur le Secteur, mais fur le banc qui l'arrête par en bas; " \& qui pouvoit lui tranfmettre une partie du coup: je ne fais " pas même s'il ne fit que du vent; car une muraille, dans un " autre endroit de la maifon, s'écroula pendant la nuit; deux 2s pendules, que nous avions dans l'obfervatoire, fe dérangèrent, „\& la révolution diurne de l'une, réglée fur le temps moyen, * changea de près d'uné minute. Tout cela nous fit craindre quel" que changement dans l'inftument, \& il y en eut un effecti» vement qui fut tel, que quoiqu'il n'intéreffât en rien Ia foli2o dité ou la fermeté de fes parties, comme je le reconnus par " l'examen que j'en fis, \& par la manière dont le moindre coup " d'ongle faifoit frémir tout le Secteur, l'étoile qui paffoit tôn» jours au deffus du fil fixe du Micromètre, paffa enfuite en » deffous, lorfque le limbe fut tourné vers l'occident. Ainfr » nous fûmes obligés de tirer un premier réfultat des huit obfer"vations que nous avions: elles nous apprirent que notre arc " de $2^{\mathrm{d}} \mathrm{S} \mathrm{I}^{\prime} S 4 \frac{\mathrm{I}}{4}^{\prime \prime}$ étoit trop petit de 5 o parties du Micromètre, s qui valent prefque $22^{\prime \prime}$, le double de la difance apparente 
DEGRES DU 'MERIDIEN. I63

de l'étoile $\varepsilon$ d'Orion au zénith étoit donc de $2^{d} 52^{\prime}$ I 6 ", \& " cette diftance de $\mathrm{I}^{\mathrm{d}} 26^{\prime} 8^{\prime \prime}$, qu'il faut augmenter de I" pour la " réfraction; le temps nous a enfuite été extrêmement contraire, " jufque-tà quon a eu beaucoup de peine à régler la Pendule. *

Secondes obfervations.

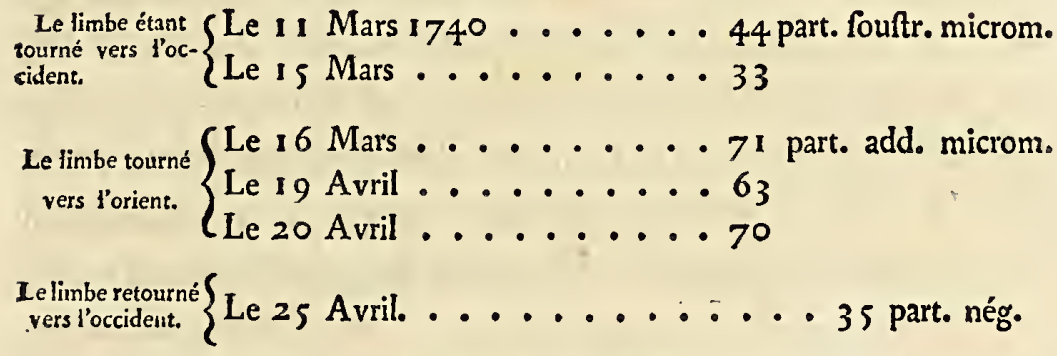

Nous trouvons par les fecondes obfervations, quil ne fau- is 'droit ajoûter que 3 I parties, qui valent, à très-peu près, I $3 \frac{1}{2}$ “ fecondes, à notre arc de $2^{\text {d }} S$ I' $^{\prime} 54 \frac{1}{4}^{\prime \prime}$ : il ne viendroit donc a que I ${ }^{d} 6^{\prime} 4^{\prime \prime}$ pour la diftance apparente de l'étoile au zénith, * \& I I $^{\prime} 6^{\prime} 5^{\prime \prime}$. pour la diftance vraie. Plufieurs raifons nous “ obligeroient, ce femble, à déférer un peu plus à ce fecond * réfultat qu'au premier; outre que toutes les obfervations qui “ nous le fourniffent ont été faites de jour, fans qu'il ait été “ néceffaire d'éclairer les fils, la détermination eft plus complète, « l'inftrument ayant été tourné \& retourné, \& nous fommes “ fûrs qu'il n'y a point eu de dérangement dans l'intervalle. Mais “ ayant mefuré le 26 d'Avril la corde de l'arc, \& l'ayant com- “ parée au rayon en la multipliant vingt fois, nous trouvâmes “ qu'elle formoit une quantité plus grande d'environ $\frac{9}{10}$ de ligne. as Nous ne démontâmes l'inftrument que le lendemain au foir, “ après avoir remefuré fon rayon, \& avoir examiné fi fon centre "s n'avoit pas changé; \& reconnu auff que toutes les parties es 
164 MESURE DES TROIS PREMIERS

* avoient toute la folidité poffible. Lorfque nous eûmes le limbe " entre les mains, nous eümes plus de commodité à bien me" furer la corde, \& nous trouvâmes encore que rép cée 20 fois, " elle formoit une quantité plus grande que le rayon; non pas. n de $\frac{9}{10}$ ligne, mais d'environ $\frac{8}{11}$ ligne. Lorfque je limitai l'arc " le 17 Février, je le fis le matin, quand la chaleur étoit à " peu près la même qu'au temps de l'obfervation, qui fe fai" foit un peu après fept heures du foir : au lieu que les compa2 raifons des $26 \& 27$ Avril fè firént dans le temps de la journée » où le limbe, qui étoit fait de cuivre rouge, avoit dû acquerir " par la chaleur, quoique toûjours à l'ombre, le plus d'exten" fion qu'il fe pouvoit, par rápport à l'affemblage des barres " de fer qui formoient le rayon : ainft cette extenfion peut „avoir eu quelque part à la différence que nous avons trouvée. „Il faut néanmoins que le dérangement que fouffrit l'inftruss ment le ro Mars, y ait beaucoup plus contribué : car à peine "Ia chaleur devoit - elle caufer une différence d'un fixième ou „ d'un feptième de ligne fur la longueur totale; mais il n'importe „ que le changement vienne de l'une ou de l'autre caufe : nous „2 l'avons remarqué à peu près vers le temps où fe faifoient alors "les obfervations, \& il faut donc y avoir égard; car tous nos „ examens réitérés tendent à le confirmer unanimement \& fans s) variation fenfible. L'arc étant trop grand d'environ $\frac{4}{110}$ ligne, „il doit valoir, vû les dimenfions de notre Secteur, environ - $4 \frac{1}{2}$ " plus que nous ne l'avions fuppofé, \& c'eft un peu plus "de 2 fecondes à ajoûter au fecond réfultat; ce qui donne $\mathrm{I}^{\mathrm{d}}$ " $26^{\prime} 7^{\prime \prime}$ pour la diftance vraie de l'étoile au zénith. Comme „s cette feconde détermination ne diffère après cela que de 2 fe2 condes de la première I ${ }^{d} 26^{\prime} 9^{\prime \prime}$, il devient indifférent, ou de 
s'arrêter au milieu, ou de déférer un peu plus à la feconde. Nous prenons I d $26^{\prime} 8^{\prime \prime}$; \& telle étoit la diftance vraie du « côté du Sud de l'étoile $\varepsilon$ d'Orion au zénith de Cotchefqui, vers « le milieu du mois dernier.

En prenant le milieu entre les obfervations faites à Tar- « qui, ce qui me paroît le parti le plus fûr après y avoir mieux a penfé, \& vû auffi l'obfervation que $M$. de la Condamine «. obtint le I 3 Janvier, l'étoile étoit éloignée du zénith de a Tarqui, du côté du Nord, de $\mathrm{I}^{\mathrm{d}} 4 \mathrm{O}^{\prime} 35^{\prime \prime}$, \& tout l'arc de la a Méridienne, compris entre les obfervatoires de Tarqui \& de « Cotchefqui, eft de $3^{\text {d }} 6^{\prime} 43^{\prime \prime}$. II ne refte plus qu’à augmenter ce cette quantité du changement qu'a pû recevoir la déclinaifon " de l'étoile depuis le commencement de Janvier de cette année, a jufque vers le i $s$ de Mars : ce changement eft très-connu, \& c d'ailleurs la fituation de l'étoile le rend très-petit. II faut auffi $\propto$ avoir égard aux changemens qui peuvent naitre ou de la paral- « laxe de l'orbe annuel, ou de la nutation de l'axe même de la s Terre. Je propofai à M. Godin, étant à̀ Cuenca, de faire pointer "s fur l'étoile une lunette rendue fixe, \&. fcellée contre un mur, « pendant le cours de nos obfervations, afin de voir les varia- « tions auxquelles l'étoile feroit fujette: il me' répondit qu'il y “ avoit déjà penfé, qửil fe chargeoit de l'exécution.... C'eft « une chofe à examiner; mais comme on le peut faire par-tout, « \& que d'ailleurs il y a lieu de croire que tous ces change- a mens font à peine fenfibles, puifqu'ils ont échappé aux yeux ri de plufieurs Aftronomes auffi attentifs qu'habiles, nous avons is cru devoir terminer nos opérations an Pérou, of regarder lobjet as de notre miffion comme entièrement rempli. FA IT à Quito, auff- a tôt mon retour du Signal de Tanlagóa', le 6 Mai 1740 . ir Signé BoUGUER: Au deffous eft écrit n: 
Je certifie les faits contenus dans le préfent écrit, de la "plufpart defquels jai été témoin \& participant, les obferva"tions ayant été faites alternativement entre M. Bouguer \& moi. „Je n'ai pû me rendre que quelques jours après $M$. Bouguer à "Cotchefqui, ayant été retenu à Quito, \& obligé de vaquer, en " qualité d'exécuteur teftanıentaire, à des affaires qui intéref" foient In mémoire de feu M. Seniergues Chirurgien de la Com"pagnie, \& l'honneur de la Nation; ainf je n'ai pas affifté » aux difpofitions préliminaires qui ont précédé la monture de „l'inftrument \& de la lunette, perfuadé d'ailleurs que je ne pouw vois mieux faire que de m'en rapporter entièrement à̀ $M$. » Bouguer; mais j'arrivai à Cotchefqui avant la première obfer» vation, \& je crus que pour pouvoir dépofer des faits avec " connoiffance de caufe \& comme témoin oculaire, je devois "vérifier par moi-même la grandeur du rayon de notre Sec" teur, \& fon rapport à la corde de l'arc qui devoit mefurer Ia - diftance de l'étoile au zénith; ce que j’exécutai le r 8 Février » dernier. Je trouvai le rayon égal à vingt fois la corde, fans dif» férence fenfible de la mefure que M. Bouguer lui avoit pro》 curée, laquelle fut auffi vérifiée dans le même temps par „M. Verguin; ce qui fert de confrrmation à l'exactitude de cette „ première mefure. II n'eft pas moins certain (quelle qu'en puiffe " être la caufe) que le rayon s'eft trouvé plus court de près de » $\frac{3}{4}$ de ligne, quand nous le remefurâmes les $27 \& 28$ Avril » dernier après notre dernière obfervation. Nous avons cherché • à douter d'un fait auquel nous nous étions fi peu attendus; » ce qui a rendu nos examens plus fcrupuleux, \& nos doutes » n’ont cédé quà l'évidence. J'ofe même affurer que la quan* tité dont nous avons jugé le rayon raccourci, n’elt nullement exagérće, nọ plus quę la différence que je trouvai à 
Tarqui le I 5 Janvier dernier dans notre première mefure, « que j'eftimai alors de $\frac{28}{100}$ de ligne, \& dont j’ai fait mention « dans le rapport ou procès verbal de notre obfervation. Enfm « ce n'eft qu'après nous être vûs for'cés d'admettre ce change- « ment de longueur du rayon, que nous avons fait réflexion * qu'il rapprochoit nos deux déterminations. Quant à ce que « nous n'appliquons qu’à la dernière l'équation qui réfulte de “ cette variation, indépendamment des raifons rapportées par « M. Bouguer, qui portent à croire que le changement s'eft fait « (du moins la plus grande partie) tout-à-coup, \& entre les “ deux obfervations, il eft certain que quand on voudroit fup- a pofer qu'il s'eft fait fucceffrvement \& proportionnellement au « temps écoulé entre les deux obfervations du i 9 Février \& « du 25 Avril, ce qui femble ne pouvoir fe concilier avec Ia « folidité de l'affemblage de toutes les parties de l'inftrument, « que nous reconnûmes en le démontant : la première déter- “ mination ne feroit pas fenfiblement affectée du changement “ fuppofé fucceffif; au lieu que la dernière, tirée d'obfervations “ dont le cours a duré près de deux mois, fe trouveroit chargée * de prefque tout l'effet produit par le raccourciffement du rayon. « 'A Quito, le $6 \mathrm{Mai}$ 1740. Signé L A CONDAMINE. Ent- « fuite eft écrit ce qui fuit:

Je foufligné, certifie la vérité de tous les faits ci-deffus « rapportés, tant pour avoir affifté à tout dès le commence- * ment, que pour y avoir aidé. J'ai eu auffi le loifir de regarder “ à la lunette dans plufieurs obfervations. FAIT à Quito, le « 6 Mai 1740. Signé VERGUIN 


\section{A R T I C L E X III.}

\section{Table d'Obfervations de l'E'toile \& d'Orion, faites en commun à Cotchefqui on 1740 .}

Réduites au premier Janvier 1743 , 'G au nouvel ob fervatoire, $14 \frac{2}{2}$ toifes plus au Nord qu'en 1740. Première Suite d'Obfervations, dans lefquelles l'arc étoit de $2^{\mathrm{d}} 5 \mathrm{I}^{\prime} 54^{\prime \prime}, 2,2 \sigma^{\top}$ fa corde $\frac{\mathrm{x}}{2 \mathrm{o}} d u$ rayon.

\begin{tabular}{|c|c|c|c|c|c|c|}
\hline $\begin{array}{c}\text { Situation } \\
\text { du } \\
\text { Secteur. }\end{array}$ & $\begin{array}{c}\text { D A T E } \\
\text { des } \\
\text { Obfervations. }\end{array}$ & $\begin{array}{l}\text { QUANTITE'S } \\
\text { obfervées avec } \\
\text { le Micromètre }\end{array}$ & $\begin{array}{l}\text { E'QUA } \\
\text { Préceffion } \\
\text { des } \\
\text { E'quinoxes }\end{array}$ & $\begin{array}{c}\text { Aberration } \\
\text { de la } \\
\text { Lumière. }\end{array}$ & $\begin{array}{l}\text { Nutation } \\
\text { de l'Axe } \\
\text { terreltre. }\end{array}$ & $\begin{array}{l}\text { Obfervations } \\
\text { réduites au I } \\
\text { Janv. I } 743 .\end{array}$ \\
\hline $\begin{array}{l}\text { Ie limbe } \\
\text { tourné } \\
\text { ̀े 'orient. }\end{array}$ & $\begin{array}{l}9 \text { Fevr. } 1740 . \\
1 \\
2\end{array}$ & $\begin{array}{l}+12^{\prime \prime}, 3 \\
+\quad 9,6 \\
+\quad 7,4\end{array}$ & $\begin{array}{l}-9,0 \\
-9,0 \\
-9,0\end{array}$ & $\begin{array}{l}-5,8 \\
-5,9 \\
-5,9\end{array}$ & $\begin{array}{r}8^{\prime \prime}, 4 \\
-8,4 \\
-8,4\end{array}$ & $\begin{array}{l}-101,9 \\
-\quad 13,7 \\
-\quad 15,9\end{array}$ \\
\hline Poccident & $\begin{array}{l}1 \text { Mars } 1740 . \\
2 \\
5 \\
8 \\
9\end{array}$ & $\begin{array}{r}+12,7 \\
+\quad 9,2 \\
+14,5 \\
+12,3 \\
+12,3\end{array}$ & $\begin{array}{r}8,9 \\
-8,9 \\
-8,9 \\
-8,8 \\
-8,8\end{array}$ & $\begin{array}{r}-6,4 \\
-6,5 \\
-6,6 \\
-6,7 \\
-6,7\end{array} \mid$ & $\begin{array}{r}8,2 \\
-8,2 \\
-8,2 \\
-8,1 \\
-8,1\end{array}$ & $\begin{array}{r}-10,8 \\
-\quad 4,2 \\
-\quad 8,8 \\
-115 \\
-115\end{array}$ \\
\hline
\end{tabular}

Arc du Secteur....................... $51^{\prime} 54^{\prime \prime}, 2 \frac{2}{2}$

Double diftance au Zénith obfervée. .... 2d $51^{\prime} 29^{\prime \prime}, 3 \frac{1}{8}$ Diftance apparente au Zénith, du côté du Sud, obfervée en $1740 \ldots \ldots 2544,6 \frac{3}{4}$ Réduction à l'obfervatoire de $1743 \ldots \ldots+\ldots+0 . \ldots$ Premier Réfultat. Diftance appar. de \& d'Orion au Zénith de Cotchefqui vers le Sud, le 1 r Janv. $1743 \ldots$ I $\mathrm{d}_{2} 5^{\prime} 45^{\prime \prime}, 6$

Seconde Suite. L'arc plus grand que dans la précédente de $4 \frac{1}{2}^{\prime \prime}$ ¿ $\mathcal{T}$ par conféquent de $2^{\mathrm{d}} 5 \mathrm{I}^{\prime} 58^{\prime \prime}, 7$.

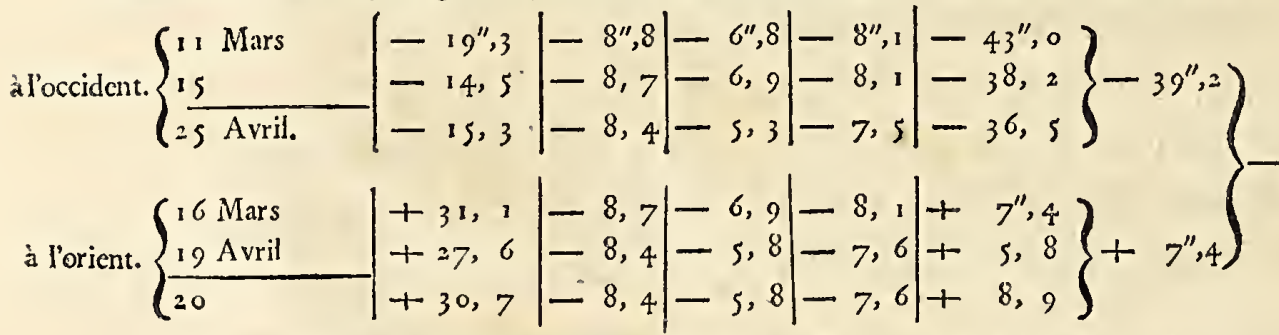

Arc du Sectcur, augmenté de 4 ", 5 par l'examen du 29 Avril. . . . . . 2 2d $51^{\prime} 58^{\prime \prime}, 7$

Double diftance au Zénith obfervée. ....2 5126,9

Diftance apparente au Zénith, du côté du Sud, obfervée en $1740 \ldots 12543,5$

Réduction à l'obfervatoire de $1743 \ldots \ldots+$

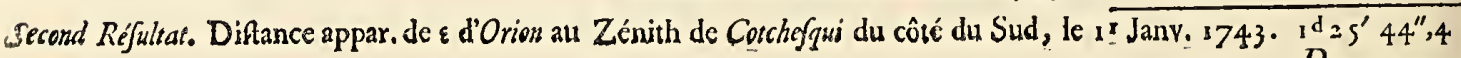


Remarques fur les objervations de la Table précédente.

Ces obfervations nous avoient donné d'abord i ${ }^{d} 26^{\prime} 7^{\prime \prime}$ pour la diftance apparente moyenne de l'étoile $\varepsilon$ d'Orion au zénith de Cotchefgui, en 1740 (p.I 64 \& I 6 5): il faut en re-

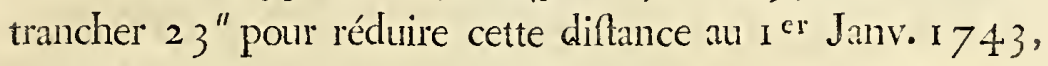
toutes les équations fe trouvant négatives; comme on le voit en détail par la Table précédente. On trouvera donc la diftance au zénith de Cotchefqui de $\mathrm{I}^{\mathrm{d}} 25^{\prime} 44^{\prime \prime}$ pour le premier Janvier I $743, \&$ de I $25^{\prime} 45^{\prime \prime}$ quatorze toifes \& demie plus au Nord, à l'endroit où $M$. Bouguer opéra, dans le temps de nos obfervations correfpondantes \& fimultanées.

On a vû par le procès verbal des obfervations faites à Cotchefqui en 1740 , que les fourchettes de fept pouces qui portoient la lunette avoient été accourcies de moitié depuis les premières obfervations de 1739 ì Tarqui : mais le nombre n'en avoit pas encore été augmenté; \& l'inconvénient, dont j’ai examiné les fuites dans l'article XI, pouvoit fubfifter en partie. II devoit feulement être fort diminué : auffi ne trouvâmes-nous en I 740, la diftance au zénith trop petite, à Cotchefqui, que de quelques fecondes; au lieu que nous l'avions trouvée en I 739 à Tarqui, de 19 \& de 27 fecondes moindre que la véritable.

Ce ne fut qu'en Septembre $174 \mathrm{I}$, Iorfque M. Bouguer multiplia le nombre des fupports de la lunette, que le Secteur acquit toute Ia folidité néceffaire. Du moins c'eft depuis cette époque, que toutes les obfervations faites à différentes xeprifes avec cet infrument, quoique par divers Obfervateurs \& fur différens arcs, ont commencé à domer conftamment une même diftance au zénith; ce qui a continué pendant le 
refte de $174 \mathrm{I}$, en $1742 \& 1743$, prefque fans variation, autre que celle qui réfulte de la fomme des équations pour la préceffion des équinoxes, \& l'aberration de la lumière. C'eft ce qui fera prouvé évidemment en fon lieu.

Quoi qu'il en foit, la diftance de l'étoile $\varepsilon$ d'Orion au zénith de Cotchefqui, obfervée en I $740, \&$ réduite au I er Janvier I 743 , ne différera que de 3 ou 4 fecondes de celle qui réfulte de nos obfervations fimultances; \& cette petite différence, diftribuée fur notre arc de 3 degrés 7 minutes, ne feroit guère que d'une feconde par degré.

De-là on pourroit être tenté de conclurre qu'il n'étoit pas néceffaire de répéter nos obfervations à Cotchefqui; mais après l'expérience d'une erreur de 23 fecondes à Tarqui, dans le moyen réfultat de trois Suites d'oblervations, que nous regardions comme exactes (Voy. Proc. verb. page I 35), nous étoit - il permis de préfumer qu’il n'y auroit rien à réformer à celui de Cotchefqui, tiré de deux Suites feulement d'obfervations, dont l'une même eft incomplète? Ce n'étoit qu'en les répétant avec de nouvelles précautions, que nous pouvions juger du degré de foi qu'elles méritoient; \& les raifons qu'il y avoit d'ailleurs (Voy. Introd. hiftoriq. à l'année I 742) pour obferver en même temps aux deux extrémités de l'arc du Méridien, étoient fi fortes, que nous n'euffions pas été excufables, fi nous euffions négligé de donner à notre détermination de la valeur du degré, un caractère fingulier d'authenticité, en prenant une précaution qui n'avoit jamais été employée, qui, de l'aveu même de M. Bonguer (Mém. de l'Acad. 1744 . pages 292 \& 293), tranche toutes les difficultés. 


\section{A R T I C L E X I V.}

Obfervations diverfes de l'étoile \& d'Orion, faites à Quito, che deux differens endroits; en $1737,1740,1741$ \& 1742 .

Réduites au premier Janvier 1.743, ¿ó à la Tour de la Mercy.

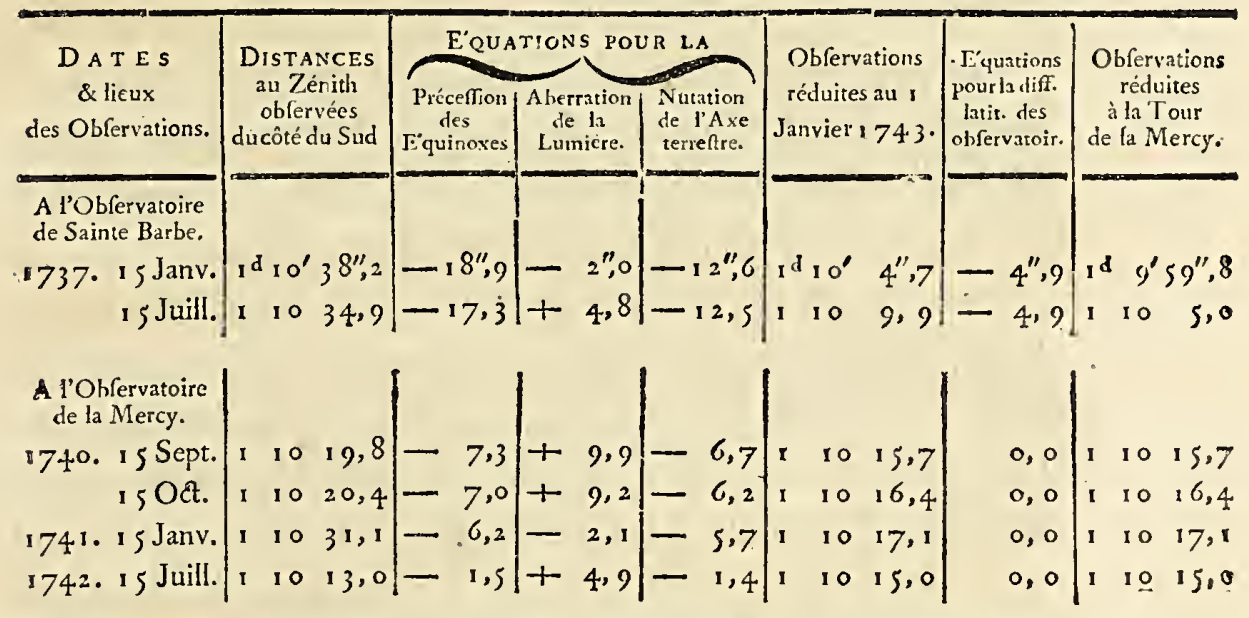

Remarques fur les obfervations de la Table précédente.

Je me fuis contenté de marquer dans la Table précédente, le réfultat de cinq différentes Suites d'obfervations, faites à Quito en différens temps \& par différens Obfervateurs. Il eut été trop long de rapporter en détail toutes les oblervations dont chaque Suite étoit compofée. Les dates ont été fixées dans la Table, à un jour à peu près également éloigné du commencement \& de lafin du temps où l'on a obfervé : par exemple, on a daté du is Janvier les obfervations faites dans le cours de Janvier; \& ainf des autres.

Voici quelques remarques fur chacun de ces réfultats en particulier; \& fur-tout à l'égard des deux premiers, qui dans 
172 MESURE DES TROYS PREMIERS

la Table font rapportés au i $s$ Janvier \& au is Juillet 1737 . L'un \& l'autre ont été tirés d'oblervations qui nous font communes, à M. Godin, M. Bouguer \& moi , \& qui nont pas été faites dans le deffein formel d'en conclurre la valeur du degré. Nous n'avions alors pour but que de vérifier la pofttion de la Iunctte du Secteur, après l'obfervation des Solftices de Décembre 17368 Juin I 737 ; mais comme nous obfervâmes pour cette vérification la diftance verticale de l'étoile \& d’Orion, la niême qui a toûjours été obfervée depuis, pour détermin:er l'amplitude de l'arc du Méridien, l'on peut faire fervir ces premières obfervations au même ufage.

Notre Secteur de douze pieds de rayon, avec lequel elles furent faites en 1737 , étoit en ce temps-là tel que nous lavions apportć de France à Quito, pour pouvoir obferver, fous l'Équateur même, le Soleil dans les deux Tropiques, \& en conclurre l'obliquité de l'Écliptique. Le limbe comprenoit alors un arc de 30 degrés, divifé en minutes. L'inftrument étoit fufpendu vers fon milieu par un genou cylindrique, comme un Quart-de-cercle ordinaire, \& porté fur un pied deftiné à un de ces inftrumens.

Quelque foin que nous puiffions dans le temps de ces premières obfervations pour faire répondre le fil-à-plomb précifément fur un point de la divifion, il n'étoit pas poflible de l'y maintenir long-temps. Il y a bien de l'apparence que le poids confidérable du total des pièces dont étoit alors formé le Secteur, faifoit quelquefois céder infenfiblement les vis de fon. pied, trop fo ble pour le corps de l'inftument. D'ailleurs, la nature de fa fufpenfion par un feul point, \& la grande furface que le limbe \& fes arc-boutans préfentoient au vent, 
auquel le Secteur étoit fort expofé, le rendoient fujề à une trépidation qui fe renouveloit \& augmentoit à la moindre agitation de l'air.

En effet, je ne crois pas que dans le temps dont je parle, nous ayons confommé une feule obfervation, fur un des points marqués fur le limbe, de minute en minute, defquels feuls nous avions compté faire ufage: \& nous n'avons pû nous paffer ${ }^{-}$du fecours des tranfverfales; auxquelles l'uuvier n'avoit pas apporté, à beaucoup près, la même attention qu'aux points.

Toutes ces raifons ont fouvent rendu notre eftime incertaine; \& elle l'eût été bien davantage, fr elle n'eût été faite à l'inftant même de l'obfervation. Tandis que M. Godin avoit l'œil à la lunette, \& tournoit l'index du Micromètre jufqu’à ce qu'il eût atteint l'étoile avec le fil mobile, nous nous relevions alternativement, M. Bonguer \& moi, pour eftimer Ia minute \& la feconde où répondoit le fil-à-plomb. Je n'ai donc pas balancé à préférer le nombre eftimé dans ce moment, à celui que nous avons quelquefois tous vî, une ou deux minutes après, \& qui différoit du premier de plufieurs fecondes, une fois entr'autres de 10 "très-évidemment.

Deux autres circonftances peuvent encore nous faire naittre quélque doute fur l'exacti ude des deux premiers réfultats de la Table. Premièrement, les obfervations fur Iefquelles ils font fondés ont été faites avec l'ancien Secteur de 30 degrés, fur des divifions tracées par l'ouvrier, \& defquelles nous ne pouvions pas répondre; au lieu que toutes les autres obfervations ont été faites fur des arcs tracés par nous - mêmes, \& dont nous connoiffions exactement la valeur. Secondement, le Secteur n'a jamuis été tourné qu'une fois pendant le cours 
de chacune de ces deux Suites d'obfervations; au lieu que dans toutes les autres, l'inftrument a non feulement été détourné, mais remis au moins une fois dans fa première fituation.

Si à toutes ces caufes d'erreur, particulières aux deux réfultats de Janvier \& de Juillet 1737 , on joint celles qui font expofées dans les articles V III \& fuivans, \& celles qui nous reftent à examiner; au lieu d'être furpris que deux obfervations, qui ont précédé toutes les autres de plufieurs années, \& qui ont été faites avec un autre infrument, ne s'accordent pas parfaitement avec les fimultanées, auxquelles tout nous engage à donner la préférence; on s'étonnera peut - être avec raifon qu'elles ne s'en éloignent que de quelques fecondes.

Au refte le réfultat du is Juillet eft celui des deux de I 737, qui approche le plus cú réfultat des obfervations fimultanées. Orion paffoit alors de jour, \& l'on ne fut pas obligé d'éclairer les fils de foie* du foyer de la lunette: ce qui, tout le refte étant égal, doit vrai-femblablement avoir rendu cette demière ob́érvation plus fûre que la précédente; \& d'autant plus qu’au mois de Janvier, on éclairoit les fils du Micromètre d'une manière incommode, en approchant de l'objectif une bougie qu'on étoit obligé de placer prefque dans l'axe de la lunette; \& qui déroboit fouvent la vûe de l'étoile. D’ailleurs cette bougie, malgré l'enveloppe de papier dont on tâchoit de lui faire un abri, étoit expofée à l'action du vent, \& fa flamme agitée caufoit dans la lunette des réfractions irrégulières. Dans

* Au lieu de fil d'argent, notre Micromètre n'étoit garni que de fils de foic fimples, moins folides, mais plus commodes pour obferver de jour; en ce qu'étant beaucoup plus fins que ccux d'argent, ils ne cachent pas l'étoile par leur épaiffeur, même en plein jour. 
toutes les obfervations poftérieures, les fils ont été éclairćs plus commodément, par une ouverture faite au bas de la Iunette, proche de l'oculaire.

Je ne dois pas non plus oublier de remarquer, que les obfervations de la diftance d'E d'Orion au zénith de Quito, au mois de Janvier I 737 , furent notre coup d'effai en ce genre, \& qu'elles ont, pour ainfi dire, fervi de prélude à toutes celles de meme nature, qui nous ont depuis $f_{1}$ fort exercés jufques en 1743 . Au refte, je ne pûs affifter à celles dont il eft ici queftion que jufqu'au I 9 Janvier 1737 que je partis pour Lima* ; mais j'en revins à temps, pour prendre part aux obfervations du Solftice de Juin de la même année.

Il a fallu ajoûter près de $\mathrm{s}$ fecondes pour réduire les deux diftances de l'étoile au zénith, en Janvier \& Juin I 737, au même lieu que les quatre fuivantes, qui ont été obfervées en I 740, I 74 I \& I 742 : parce que l'endroit où ont été faites les premières obfervations, \& qui eft marqué $K$ fur le plan de Quito, eft plus feptentrional de 75 toifes que la Tour de In 'Mercy; \& que le centre de cette Tour n'eft que de deux toifes plus Nord que le point $L$, où nous avons toûjours obfervé depuis I 737. Au refte, cette équation de s", au lieu de rapprocher les réfultats des obfervations de 1737 de ceux des années fuivantes, les en éloigne davantage.

Les quatre derniers réfultats de la Table font tirés d'obfervations poftérieures au changement de forme \& de fufpenfron de notre Secteur, \& même aux obfervations de Tarqui \& de Cotchefqui en I 739 \& I740, rapportées dans les articles

* Pour y aller chercher des fecours qui pûfent nous mettre en état de continuer nos opérations. 


\section{IT MESURE DES TROIS PREMIERS}

précédens, \& que nous avons depuis abandonnées. Les trois réfultats qui ont pour date les is Sept. is Octobre $1744^{\circ}$ \& is Janvier 174I, font de M. Bouguler. Je ne rapporte point celui d'une obfervation que javois fort avancée au mois de Décembre 1740; parce qu’elle ne fut pas terminée. J'eus lieu de croire, par les varintions que je remarquai les derniers jours, que le Secteur avoit fouffert quelque dérangement; mais je ne pûs achever de m'en convaincre, M. Bouguer ayant touché à l'objectif, pour mettre la lunette à fon point, te jour que je devois retourner l'inftrument.

Le dernier réfultat, en date du is Juillet 1742 , eft de moi. J'avois commencé à obferver dès le mois de Mai; mais je perdis de vûe l'étoile dans les rayons du Soleil avant que d'avoir tourné le Secteur, \& je n’ai tiré la diftance au zénith employcé dans la Table, que des obfervations faites depuis que l'étoile eut recommencé à paroître. Celles-ci l'ont été en plein jour, \& fans qu'il fût befoin d'éclairer les fils. On voit par la Table que les quatre dernières diftances au zénith, réduites à une même époque, ne diffèrent pas entr'elles de $3^{\prime \prime}$; \& que les différences de 7, 8, \& i 8 fecondes, qui fe trouvent entre mon obfervation de Juillet 1742 , \& celles de M. Bouguer en 1740 \& $174 \mathrm{I}$, un an \& demi ou deux ans alparavant, s'évanouiffent prefque entièrement par la réduction à la même date.

L'accord de ces quatre réfultats, rend les deux précédens tirés des obfervations de Janvier \& Juin I 737, d'autant plus fufpects, que ceux-ci difièrent des autres de plus de dix fecondes. Une erreur dans la divifion de l'ancien limbe, de Isquelle nous ne pouvons répondre comme des arcs que nous 
avons depuis tracés nous-mêmes, pourroit caufer toute cetie différence. Quoi qu'il en foit, fi l'on prend un milieu entre les fix réfultats de la Table précédente, qui font tirés d'obfervations faites à Quito dans le cours d'environ fix ans, par divers Obfervateurs; on aura la diftance apparente de l'étoile $\varepsilon$ d'Orion au zénith de la Tour de Ia Mercy de Quito de I d I o' I I", 4 pour le premier Janvier I743; \& fi à cette quantité on ajoutte celle de I $S^{\prime} 4 I^{\prime \prime}$ pour la réduire à notre obfervatoire de Cotchefqui, lequel eft I 4839 toifes plus Nord que 1 a même Tour* ${ }^{*}$ on trouvera la diftance apparente de l'étoile au zénith, de I $25^{\prime} 52^{\prime \prime}$, toute réduction faite; ce qui ne diffère guère, comme on le verra bien - tôt, du réfultat des dernières obfervations de M. Bouguer à Cotchefqui à la fin de 1742.

Ce n'eft qu'à force de faire fucceffivement des changemens à notre Secteur pour le rendre plus folide, \& à force de prendre les nouvelles précautions qu'une expérience continuelle nous fuggéroit de jour en jour, que noš obfervations parvinrent par degrés à acquerir cette uniformité, que nous avions en vain defirée dans les anciennes.

C'eft de quoi fourniffent une nouvelle preuve les obfervations même de la Table fuivante, dont les trois dernières Sụites, poftérieures au mois de Septembre I 74 I , donnent les premiers réfultats, fur lefquels nous pouvons faire fond.

* Diftance du Signal de Cotche fqui à la perpendiculaire à la Tour de Ia Mercy de Quito, réduite au niveau de Carabourou, 148 I 2', 9 I (Part. I, art. XVIII, $p .68$.); plus '25t,06, dont l'obfervatoire de Cotcliefqui étoit plus Nord que le Signal en 1742 ( $p .103)$ : plus I toife pour l'excès de la hauteur moyenne du terrein entre Cotchefqui \& Quito, au deffus de Carabourou, évalué à 250 toiles (Part. I, art, XIY,p. 55 \& $5 \sigma$ ). 


\section{A R T I C L E X V.}

\section{Table des Obfervations de l'étoile \& d'Orion, faites à Tarqui en $I 74 I$, par $M$. Bouguer,}

Sur un arc de 3"2 2" 22": dont la corde étoit de 7 "plus grande que la $17^{\circ}$ partic du rayon.

Riduites au premicr Janvior 1743 .

Premiere Suite d'Obfervations.

\begin{tabular}{|c|c|c|c|c|c|c|c|c|}
\hline $\begin{array}{c}\text { Situation } \\
d \text { du } \\
\text { Secteur. }\end{array}$ & $\begin{array}{c}\text { D A T E } \\
\text { des } \\
\text { Obfervations. }\end{array}$ & $\begin{array}{l}\text { Quantite's } \\
\text { obfervées avec } \\
\text { le Micromètre. }\end{array}$ & $\overbrace{\begin{array}{l}\text { Préceffion } \\
\text { des } \\
\text { E'quinoxes }\end{array}}^{\text {E'QUA }}$ & ATIONS POL & $\begin{array}{l}\text { Nutarion } \\
\text { de l'Axe } \\
\text { terreftre. }\end{array}$ & $\begin{array}{c}\text { RE'DUCTION } \\
\text { au \& Janvier } \\
\text { ' } 743 .\end{array}$ & MILIEUX. & RE'SULTATS \\
\hline $\begin{array}{l}\text { 1.e limbe } \\
\text { tourné. } \\
\text { à l'orient. }\end{array}$ & 5 Mars 1741. & $-2^{\prime} 20^{\prime \prime}, 3$ & $+s^{\prime \prime}, 7$ & $+6^{\prime \prime}, 6 \mid$ & $+5^{\prime \prime}, 3$ & - 2'02".7 & & \\
\hline
\end{tabular}

Double diftance au Zénith, obfervée ..... $3^{\mathrm{d} 22^{\prime}} 53^{\prime \prime}, 3$

Premier Réfultat. Dift. appar. de \& d'Orion au Zénith de Tarqui vers le Nord, au , cr Janv. $1743 \ldots \ldots$ I 4126,6

On fait plufreurs changemens au Seateur. Seconde Suite d'Objervations.

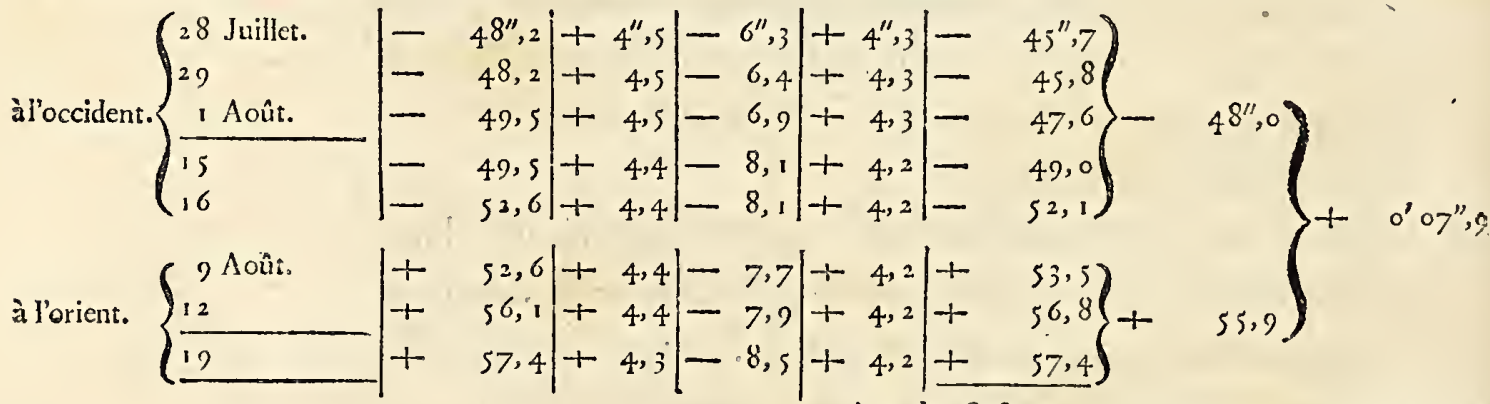

Arc du SeAeur............ 32222 ,

Double diftance au Zénith, obfervée. . . . . 3 $3^{d} 2^{\prime} 29^{\prime \prime}, 9$

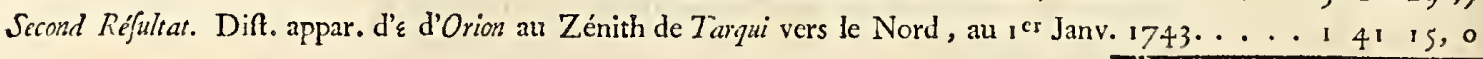

On remet de nouvelles foies au Micromètre. Troificme Suite d'Obfervations.

à P'orient. $\left\{12\right.$ Sept. $\left.\left|-23^{\prime \prime}, 2\right|+4^{\prime \prime}, 1\left|-9^{\prime \prime}, 8\right|+3^{\prime \prime}, 6 \mid-25^{\prime \prime}, 3\right\}$

al'occident. $\left\{13 \quad|+21,5|+4,1|-9,8|+3,6 \mid+2_{3,6}\right\} \cdots \cdots-\quad 1 ", 7$

A près avoir dćtourné une feconde fois l'Inftrument, M. Rouguer reconriôit par olfervation \& par mefure actuelle ๆqu'il s'eit dérangè.

Arcdu Sccteur .......... 32222,0

Jouble diftance au Zénith, obfervée. . . . . . 3 $3^{d} 2^{\prime} 20^{\prime \prime}, 3$

Troifieme Réfultat. Dift. appar. d'є d'Orion au Zénith de Tarqui vers le Nord, au $1^{6 r}$ Janv, $1743 \ldots \ldots$. . 41 10, 
On démonte le Secheur, on rive fes vis, \& on larfermit pal de nouvesux liens de fil de fer.

Quatrième fuite d'Obfervations.

\begin{tabular}{|c|c|c|c|c|c|c|c|c|}
\hline $\begin{array}{c}\text { Situation } \\
\text { du } \\
\text { Secteur. }\end{array}$ & $\begin{array}{c}\text { D A } \mathrm{T} E \\
\text { des } \\
\text { Obfervations. }\end{array}$ & $\begin{array}{l}\text { QuANTITE'S } \\
\text { obfervées avec } \\
\text { le Micromètre. }\end{array}$ & $\begin{array}{l}\text { Prćceffion } \\
\text { des } \\
\text { E'quinoxes }\end{array}$ & $\begin{array}{c}\text { Aberration } \\
\text { de li } \\
\text { Lumicre. }\end{array}$ & $\begin{array}{l}\text { UR LA } \\
\text { Nunaticn } \\
\text { de l'Axe } \\
\text { terreftre. }\end{array}$ & $\begin{array}{c}\text { RE'DUCTION } \\
\text { au I Janvier } \\
\text { I } 743 .\end{array}$ & $M \perp L I E \cup X$. & RESULTATS. \\
\hline $\begin{array}{l}\text { Le limbe } \\
\text { tourné } \\
\text { à l'orient. }\end{array}$ & 9 Oet, $174 \mathrm{~s}$ & $\begin{array}{l}+\quad 46^{\prime \prime}, 9 \\
+\quad 49,1\end{array}$ & $\begin{array}{l}+31,9 \\
+\quad 3,9\end{array}$ & $\begin{array}{r}-9^{\prime \prime}, 6 \\
-\quad 9,5\end{array}$ & $\begin{array}{l}+311,0 \\
+\quad 3,0\end{array}$ & $\begin{array}{l}+\quad 44^{\prime \prime}, \\
+\quad 46,\end{array}$ & 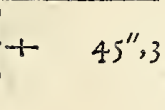 & . \\
\hline à l'occident. & 27 & $\begin{array}{r}-43,8 \\
-\quad 40,8\end{array}$ & $\begin{array}{l}+3,7 \\
+\quad 3,7\end{array}$ & $\begin{array}{r}8,4 \\
-\quad 8,4\end{array}$ & $\begin{array}{l}+\quad 2,9 \\
+\quad 2,9\end{array}$ & - & & \\
\hline
\end{tabular}

Le Limbe ayant été retourné une feconde fois yers

Orient, une obfervation de $a$ du Verfeau, au défaut

Arc du Secteur ....... 32222,0 d'\& d'Orion, prouve que le Secteur n'a point varié.

Double diftance au Zénith, obfervée ....... $3^{d} 22^{\prime} 23^{\prime \prime}, 2$

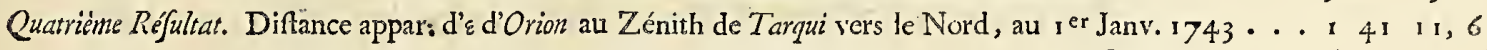

$M_{i}$ Bouguer démonte de nouveau le Secteur, il ajoûte un fupport \& de nouveatxx liens à Is Lunette.

\section{Cinquième Suite d'Obfervations.}

al'occident. $\left\{\begin{array}{l}18 \text { Nov. } \\ \frac{19}{22} \\ 23\end{array} \mid \begin{array}{l}+ \\ + \\ +\end{array}\right.$

ลे l'orient. $\left\{\begin{array}{l}20 \text { Nov. } \\ 21\end{array}\right.$

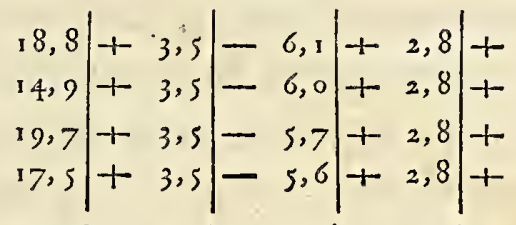

$\underset{\infty}{\infty}$

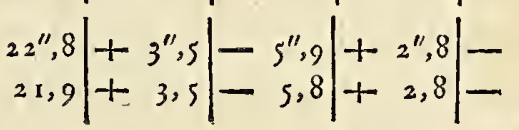

Arc du Secteur........... 32222

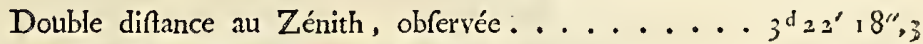

Cinquieme Réfultat, Diftance appar. d'є d'Orion au Zénith de Tarqui vers le Nord, au tar Janv. $1743 \ldots$. 1 4.1 9, 2

On fait de nouveaux changemens à Ia Lunette, on ôte le diaphräme (ou pinnule de l'oculaire), \& on le felle de nouveas.

Sixieme fuite d'Objervations.

à loccident. $\left\{\frac{2 \text { Déc. }}{4} \mid+\right.$

à l'orient. $\{3$ Déc.

$1-$

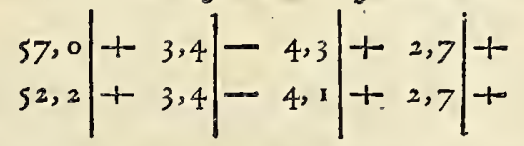

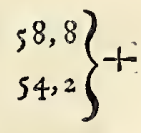

$\left.52^{\prime \prime}, 5\right\} \rightarrow$

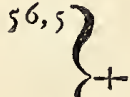

$\left.52^{\prime \prime}, 5\right)$

Arc du Secteur .......... 3 2222

Double diftance au Zénith ........ $3^{\mathrm{d}} 22^{\prime} 26^{\prime \prime}$

Siwieme Réfultat. Difance apparente d'E d'Orion au Zénith de Tarqui vers le Nord, au 1 er Janvier $1743 \ldots \ldots \ldots \ldots \ldots \ldots \ldots \ldots \ldots \ldots$ 
Remarques fur les objervations de la Table précédente.

Toutes ces obfervations mont été communiquées par $M$. Bouguer dans fa lettre de Tarqui du 5 Décembre I $74^{-1}$; je n’ai fait que leur donner la forme d'une Table, en les réduifant, comme toutes les autres, au premier Janvier I 743 .

Cette Table comprend fix Suites d'obfervations, \& fix réfultats indépendans l'um de l'autre, y ayant toûjours eu entre Ia Suite précédente \& la fuivante quelque changement fait à linftrument, foit pour le rendre plus folide, foit pour varier les procédés des obfervations. Du refte, elles ont toutes été faites fur le même arc.

L'intention de M. Bouguer avoit été de prendre pour corde Ia dix - feptième partie du rayon; mais apparemment qu'ers pefant, pour marquer les points extrêmes de l'arc, fur la tête du compas d'arrêt, ouvert de la quantité quion avoit portée dix-fept fois fur le rayon (Voyez Part. II, art. IV, page $x$
I ), les pointes firent reffort, \& s'écartèrent en gliffänt un peu au dehors de Parc. Quoi qu'il en foit, après que les rebarbes furent ufées, la diftance des deux points fe trouva trop grande, d'une quantité qui fut évaluée par M. Bouguer à 7 ": ce qui fit que l'arc au lieu de $3^{\mathrm{d}} 22^{\prime}$ i $5^{\prime \prime}$ qu'il avoit eu deffein de lui donner, fe trouva de $3^{\mathrm{d}} 22^{\prime} 22^{\prime \prime}$; tel quili a été employé dans la Table.

Le premier réfultat des \&\& I 7 Mars I 74I, saccordoit en apparence avec les fuivans, avant qu'on y eût appliqué les équations. Depuis les corrections \& la réduction à une même époque, il diffère des autres de I 4 à I 5 "; \& indépendamment de cette confidération, il ne mérite aucune foi; 
non feulement parce que l'infrument n'a pas été retourné une feconde fois, comme nous l'avons obfervé invariablement, depuis que nous eûmes remarqué la facilité qu'il avoit à fe déranger; mais encore, parce que fon dérangement en cette occafion, qui n'avoit d'abord été que foupçonné, fut depuis confrrmé \& prouvé évidemment; $M$. Bongutuer ayant reconnu, par plufieurs obfervations immédiatement fuivantes, quiil fe vit obligé d'abandonner, que le Secteur changeoit. d'état en le retournant. Je n'ai donc rapporté ce premier réfultat que pour n'en omettre aucun de ceux que nous nous fommes communiqués, \& dont le défaut n'avoit pas d'abord été manifeftement reconmu.

Le fecond \& le troifième, l'un du commencement d'Août, l'autre de la mi-Septembre, n'ont guère plus d'autorité que le précédent, \& par une raifon femblable. Le 3 o Septembre, M. Bouguer ayant retourné l'inftument une feconde fois, ne retrouva plus le même nombre qu'il avoit obfervé dans la première fituation de l'inftument ; \& de plus, il vérifia par mefure actuelle, que le Secteur s'étoit encore dérangé dans l'opération du retoumement; ce qui doit au moins rendre fufpect le réfultat précédent du mois d'Août; quelque conforme qu'il foit d'ailleurs, à ceux qui ont été tirés des obfervations faites depuis que le Secteur eut acquis toute la folidité requife.

Il faut avouer que jufqu'au temps dont je parle, nous n'avions réuffr à mettre notre Secteur à l'abri de pareilles variations, ni à Tarqui, ni à Cotchefqui, ni à Quito; \& que par' conféquent nous ne pouvons compter fûrement fur aucunes. des obfervations antérieures; mais le dérangement reconnu le. 30 Septembre 74 I dans l'affemblage des parties du Secteur, 


\section{MESURE DES TROIS PREMIERS}

fut le dernier de cette nature. Ce jour-là même, M. Bouguer travailla efficacement à remédier une fois pour toutes, at Ia facilité qu'avoit eue jufque - là cet inftrunıent à changer de figure; il le fit démonter, il en fit refferrer, \& enfuite river toutes les vis; il fit fortifiel' tout l'enfemble par des liens de fil de fer, \& par une traverfe horizontale; il ajoûta un fupport à la lumette. \& y mit nutre cela de nouveaux liens tenant lieu de fupports; pour ne faire de la lunette qu'un feul corps, avec le rayon qui la foutenoit. II recueillit bien-tôt le fruit de fes peines. Depuis ce temps, quoiqu'il ait encore dé-. monté le Secteur, \& fait entre les oblervations poftérieures divers changemens, qui faifoient varier l'axe optique de la lunette \& le nombre des parties du Micromètre; l'affemblage total du Secteur a paru inébranlable. Auff les trois réfultats fuivans s'accordent-ils, fans que l'application des équations, qui nous étoient alors inconnues, change rien à cet accord.

La moyenne diftance au zénith, tirée de ces trois réfultats, eft Id $4 \mathrm{I}^{\prime}$ I I $\frac{\mathbf{I}^{\prime \prime}}{4}$. Si on y joint le fecond, que je n'ai exclus, que parce que l'inftrument s'étoit dérangé après le troifième, la diftance moyenne au zénith diminuera feulement de $\frac{1}{4}$ de feconde. Enfin quelle que foit celle qu'on adopte, elle fera tonijours la même, à une feconde près, que ce que je trouvai un an après, "pendant un cours d'obfervations dè fix mois; que je fis au même lieu : après avoir nouvellement reconftruit le Secteur, qui, dans l'intervalle, avoit été tranfporté à Quito, \& étoit revenu à Tarqui; après avoir tracé fur le limbe un nouvel arc ; \& pour ainfi dire, après avoir formé un inftrument tout nouvean; indépendamment des nouvelles attentions que j'apportai d'ailleurs, dont je vais bien-tôt rendre compte. 


\section{A R T I C L E X V I.}

Dernières obfervations, faites à Cotchefqui, au Nord de la Mériditnne, correfpondantes à celles qui ont été faites en même temps, à l'extrémité Sud.

TA B L E des diftances de l'E'toile \& d'Orion au Zénith de Cotchefqui, obfervées par $M$. Bouguer à la fin de 1742 , \& réduites au $I^{e r}$ Janv. 1743 .

Première Suite d'Obfervations, faites fur un arc de $2^{d} .5 \mathrm{I}^{\prime} 54^{\prime \prime}, 3$, dont la corde étoit $\frac{1}{20}$ du vayon.

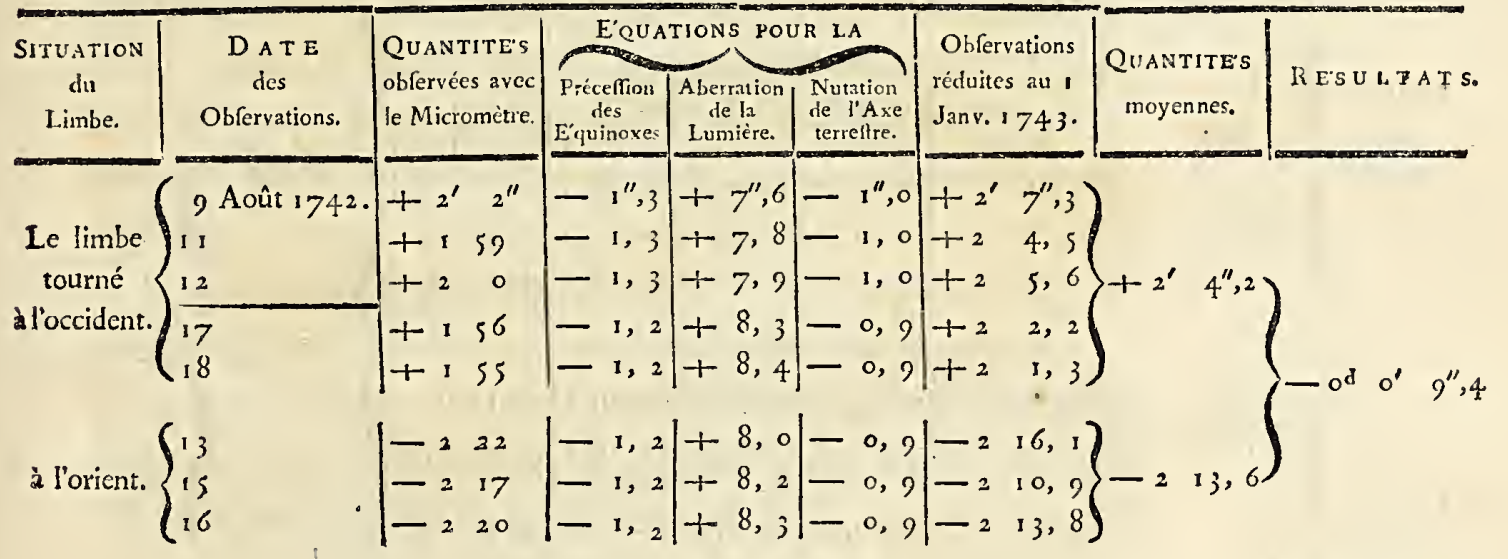

Arc du Secteur. . . . . . . . . . . 2d $51^{\prime} 54^{\prime \prime}, 3$

Double diffance d' $\varepsilon$ au Zénith, obfervée . . . . . 2 51 44, 9

Prenier Réfultat. Dift. appar. d'E d'Orion au Zénith de Cotchefqui, vers le Sud, au I $^{\mathrm{cr}} \mathrm{Janv} .1743 \ldots \ldots$ I $25 \quad 52,4$ M. Bouguer démonte l'Inftrument, change l'objectif de place, refferre les vis, \& affermit avec des fills de fer les fupports de la lunette.

\section{Seconde Suite d'Obfervations.}

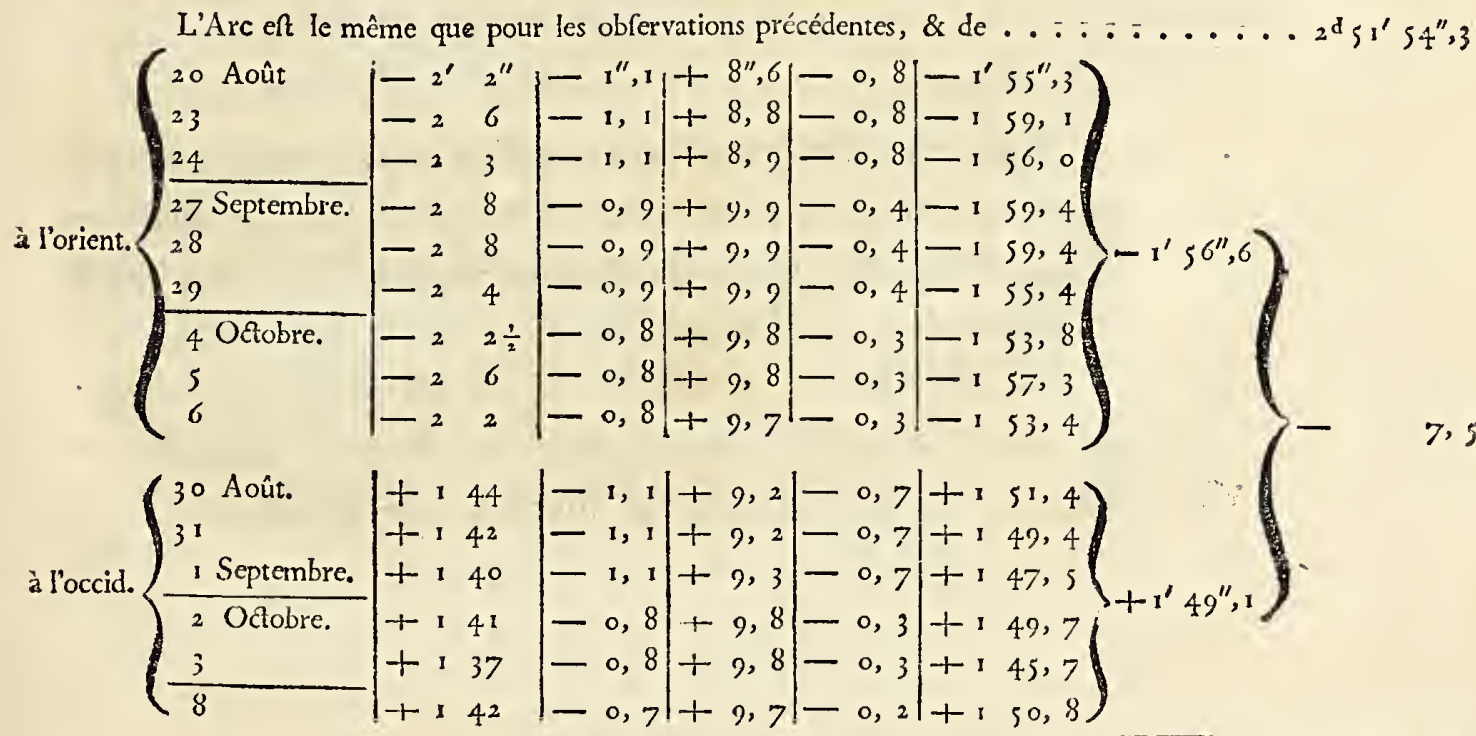

Double diftance d' $\_$au zénith, obfervée......... 2546,8

Second Refultat. Dift. appar. de \& d'Orion au Zénith de Cotchefqui, vers le Sud, au ${ }_{1}$ cr Janv. $1743 \ldots \ldots$. 2553,4 
184 MESURE DES TROIS PREMIERS

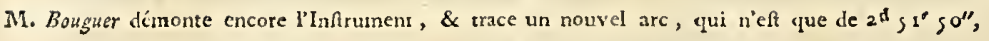

Troifieme Suite d'Obfervations.

\begin{tabular}{|c|c|c|c|c|c|c|c|c|}
\hline $\begin{array}{c}\text { Situation } \\
\text { du } \\
\text { Limbe. }\end{array}$ & $\begin{array}{c}\text { D A T E } \\
\text { des. } \\
\text { Obfervations. }\end{array}$ & $\begin{array}{l}\text { QUANTITE'S } \\
\text { obfervées avec } \\
\text { le Micromètre. }\end{array}$ & $\begin{array}{c}\text { E'QUA } \\
\text { Préceflion } \\
\text { des } \\
\text { E'quinoxes. }\end{array}$ & $\begin{array}{c}\text { A berration } \\
\text { de la } \\
\text { Lumière. }\end{array}$ & $\begin{array}{l}\text { UR LA } \\
\begin{array}{l}\text { Nutation } \\
\text { de l'Axe } \\
\text { terreftre. }\end{array}\end{array}$ & $\begin{array}{c}\text { Obfervations } \\
\text { réduites au } 1 \\
\text { Janv. } 1743 .\end{array}$ & $\begin{array}{l}\text { QUANTITES } \\
\text { moyennes. }\end{array}$ & RESULTATS。 \\
\hline $\begin{array}{c}\text { Le limbe } \\
\text { vers } \\
\text { J'occident. }\end{array}$ & $\begin{array}{l}22 \text { OEt. } 1742 . \\
26 \\
27 \\
29 \\
29 \text { Novi. } \\
30 \\
17 \text { Déc. } \\
29 \\
31 \\
23 \text { O\&. } \\
2 \text { Déc. } \\
5 \\
6 \\
8 \\
9 \\
\text { I Janv. 1743. } \\
2\end{array}$ & 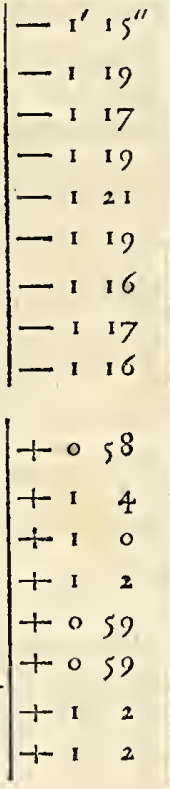 & \begin{tabular}{|l}
$\mid \begin{array}{l}0,6 \\
-0,6 \\
-0,6 \\
-0,6 \\
-0,3 \\
-0,2 \\
-0,1 \\
-0,0 \\
-0,0\end{array}$ \\
$-0,6$ \\
$-0,2$ \\
$-0,2$ \\
$-0,2$ \\
$-0,2$ \\
$-0,2$ \\
$-0,0$ \\
$-0,0$
\end{tabular} & $\left|\begin{array}{l}+8,, 8 \\
+8,5 \\
+8,4 \\
+8,3 \\
+4,8 \\
+4,7 \\
+2,2 \\
+0,5 \\
+0,2\end{array}\right|$ & $\left|\begin{array}{cc}-0,2 \\
-0,1 \\
-0,1 \\
-0,1 \\
-0,1 \\
-0,1 \\
-0,0 \\
+0,0\end{array}\right|$ & $\mid \begin{array}{ll}-1^{\prime} & 7^{\prime \prime}, 0 \\
-1 & 11,3 \\
-1 & 9,4 \\
-1 & 11,5 \\
-1 & 16,6 \\
-1 & 14,6 \\
-1 & 14,0 \\
-1 & 16,5 \\
-1 & 15,8\end{array}$ & . & 9, \\
\hline
\end{tabular}

Arc da Sccterr. ............................... $5 a^{\prime} 50^{\prime \prime}, 0$

Double diftance obfervée d' $\varepsilon$ au Zénith ........... 2 51 41 , o

Troifieme Réfultat. Diftance apparente d's au Zénith de Cotchefqui, vers te Sud ... I 25 50, 5

Remarques fur les obfervations de la Table précédente.

Nous voici enfin parvenus aux obfervations correfpondantes, qui furent faites en même temps aux deux extrémités de notre arc du Méridien, \& defquelles nous devons tirer l'amplitude de cet arc, \& la valeur du degré.

La Table précédente contient celles que faifoit $M$. Bonguer au Nord de la Méridienne, tandis que j’en faifois d’autres au Sul. Les premières ont duré depuis le 9 Août 1742 , jufqửau 2 Janvier 
Janvier I 743 : elles ont été faites à Cotchçqui avec un nouveau Secteur, conftuit à Quito tout exprès par le freur Hugo notre Horloger, fous les yeux \& fous Ia direction de M. Bougucr. Le sayon de ce nouvel inftrument étoit de huit pieds : il portoit une lunette de même longueur, qui y étoit appliquée très-folidement, \& prefque immédiatement. Sa fufpenfion étoit différente de celle de l'ancien Secteur: celui - ci étoit mobile fur un pivot prefque vertical. M. Bouguer, qui en a feul fait ufage, en aura fans doute donné la defcription \& le deffein.

Quoique M. Bouguer eut cherché à éviter, dans la confruction de ce nouveau Secteur, les défauts que nous avions remarqués dans l'ancien, \& dont nous n'avions que trop éprouvé jufqualors les conféquences; il procéda avec les mêmes précautions que s'il eût eu les mêmes obftacles à furmonter. Il démonta \& remonta plufieurs fois le nouvel inftrument, \& changea l'objectif de place; en forte que fes dernières obfervations à Cotchefqui en I 742 compofent trois Suites différentes, \& donment trois réfultats tout à fait indépendans l'un de l'autre. Outre cela, l'arc qui a fervi aux deux premières Suites, n'eft pas le même que celui fur lequel ont été faites les obfervations, qui ont fourni le troifième réfultat. Le premier arc avoit pour corde précifément la $200^{\mathrm{e}}$ partie du rayon; c'eft-à-dire, [qu'il étoit de $2^{\mathrm{d}} 5 \mathrm{I}^{\prime} 54 \frac{1}{3}^{\prime \prime}$. En marquant les points qui devoient terminer le fecond arc, il fe trouva plus court que le précédent d'une petite quantité, qui fut évaluée à plus de 4 fecondes, \& par conféquent l'arc n’étoit plus que de $2^{d} 5 I^{\prime} 5 o^{\prime \prime}$.

La difance apparente de l'étoile au zénith, tirée de la $\mathrm{Cc}$ 


\section{MESURE DES TROIS PREMIERS}

première Suite d'obfervations, eft de I $2 S^{\prime} S 2^{\prime \prime}, 4$ : elle eft de I $25^{\prime} 53^{\prime \prime}, 4$ par la feconde, \& feulement de Id $25^{\prime}$ so", 5 par la troifième. Ces différences font fi légères, furtout fi l'on confidère que les obfervations de chaque réfultat ont été faites, pour ainfi dire, avec différens inftrumens, qu'il y a peu d'erreur à craindre en prenant un milieu. Cependant M. Bouguer, en me les communiquant les unes \& les autres, me marquoit qu'il n'avoit pas cru devoir ajoûter une entière foi aux premières obfervations, \& qu'il étoit tenté de les rejeter à caufe des divers obftacles qu'il avoit eu à vaincre: en effet, cétoit un inftrument tout nouveau qu'il perfectionnoit à mefure yu'il en faifoit ufage; \& par cette raifon, les dernières obfervations, qui font aufli les plus uniformes, femblent mériter la préférence. Si donc on s'en tient, fuivant nos conventions, à celles qui ont été faites en nème temps, aux deux extrémités de l'arc; c'ent au demier rélultat $\mathrm{I}^{\mathrm{d}} 2 \mathrm{~S}^{\prime}$ 5o" qu'il faudra s'arrêter: encore faudroit-il ne le tirer que des obfervations faites depuis le 29 Novembre, que les correfpondantes ont commencé de ma part à Tarqui. La diftance apparente au zénith de Cotchefqui feroit en ce cas moindre de près d'une feconde, c'eft-à-dire, de $\mathrm{I}^{\mathrm{d}} 25^{\prime} 49^{\prime \prime}, 2$; \& il $\mathrm{y}$ en auroit encore prefque une autre à retrancher, fi on ne prenoit le nilieu des obfervations de M. Bouguer à Cotchefqui, qu’à compter du 8 Décembre; qui eft, comme je l'expliquerai en fon lieu, l'époque oà j’ai commencé à être fûr des miennes à I arqui. Ceci polé, la diftance apparente de l'étoile $\varepsilon$ d'Orion au zénith de Cotchefqui, tirée des obfervations de M. Bouguer, correfpondantes aux miennes de Tarqui, feroit de $1^{d}{ }^{\prime} 5^{\prime} 48^{\prime \prime}, 3$, en réduilant tout au premier Janvier 1743 ; 
II me refte à rendre compte de ces obfervations. Je les fis feul à Tarqui pendant que M. Bouguer faifoit à Cotchefqui celles que je viens de rapporter. Mais avant que de domner la Table \& l'examen des miennes, je crois devoir parter des moyens nouveaux que j'employai pour perfectionner le Secteur, \& pour mieux réuffir dans ces obfervations qué dans les anciennes.

\section{A R T I C L E X VII.}

Des précautions particulieres que je pris dans les dernières obfervations que je fis à Tarqui $e n 1742$ do 5743 , en correfpondance de celles que $M$. Bouguer faifoit dans le même temps, à l'autre extrénité de la Méridicne.

Secteur raffermi. Sufpenfion perfectionnée. Limbe aplani.

Quorque dans le détail fuivant je courre le rifque de me rencontrer avec M. Bouguer, \& peut-être de redire ce quil aura dit mieux que moi; cependant comme it eft ici queftion d'obfervations que j’ai faites feul à une extrémité de la Méridienne, dans le même temps que M. Bouguer obfervoit à l'autre; \& que c'eft de ces obfervations contemporaines, dont quelques-unes font fimultanées, que nous fommes convenus de déduire l'amplitude de l'arc du Méridien ; je ne puis me difpenfer de rendre un compte détaillé des précautions nouvelles que je pris en mon particulier, pour affurer le fuccès de la partie de notre travail, de laquelle je me trouvois chargé: comme M. Bouguer l'aura fait à l'égard de celle qui le concernoit. Au refte jaurai fain de paffer fous filence ce qui eft $\mathrm{C} c$ ij 
commun à ces dernières obfervations \& aux précédentes, \& tout ce que j’ai déjà remarqué ailleurs, ou dont il eft fait mention dans les procès verbaux rapportés dans les articles VI \& XII.

J'ai déjà dit que j’avois fait tranfporter à bras, de Quito à Tarqui, dans une caiffe folide \& proportionnée, notre Secteur tout monté : tel quil venoit de me fervir à Quito, pour une obfervation que j'y avois faite immédiatement avant mon départ; uniquement dans la vûe de me préparer à celles que j'allois entreprendre à Tarqui. Malgré toutes mes précautions, je m’aperçus dès que je commençai à obferver, que dans un tran!port de 8 o lieues, \& par un pays de montagnes, l'affemblage des pièces qui compofoient l'inftrument, devoit avoir fouffert quelque dérangement; puifque l'axe optique de la lunette avoit fenfiblement changé de fituation. Je ne balançå pas à démonter le Secteur, à en raffermir toutes les parties, \& à le confruire tout de nouveau; mais avant que de le démonter, j’y fis une autre réparation.

J'avois remarqué dès le temps de nos premières obfervations, que l'hémifphère $G$, par lequel le Secteur étoit fufPlanche III. pendu (Voy. art. II), ne tournoit pas affez librement dans le carcaı $K$. Nous tâchions d'y fuppléer en foûlevant l'inftument, pour faciliter fon mouvement de rotation fur fon axe, lorfqu'on le voubit diriger dans le plan du Méridien, \& fur-tout lorfquon le retournoit pour la vérification; mais il étoit toûjours à craindre que la réfiftance caufée par le frottement, jointe à la longueur de r 2 pieds qu'avoit le rayon, n'occafionnât à la barre de fer $E C$, quelque torfion en fpirale, qui changeât la fituation du rayon par xapport à la lunette. Je 
crus même, en y regardant de près, remarquer à l'oil quelque chole de femblable, quand j’eflayois à deffein d'imprimer au Secteur le mouvement fur fon axe par le demi-globe de bronze $G$, qui formoit fa fufpenfion. Pour prévenir jufqu'au foupçon de cet accident, je paffai une journée entière, aidé de $M$. de Morainville, à ufer le demi-globe fur fon collier $K$ : d'abord avec un fable noir métallique très-dur, que l'aiman attire, \& qui eft commun dans le pays*, \& enfuite avec l'émerii. Nous parvînmes à diminuer les frottemens au point que, malgré le poids de l'inftrument, on le faifoit toumer librement fur fon axe, en le pouflant du bout du doigt, par en bas à l'extrémité du limbe. Cependant, pour une plus grande fureté, ce fut ton̂jours par le haut qu'on continua de le mettre en mouvement, toutes les fois quil fut queftion de le retourner.

Après cette opération, je démontai l'inftument, je fis faire de nouveaux écrous \& de nouvelles vis; je les fis ferrer à force, \& fans y mettre d'huile, fuivant l'avis de M. Bouguer: je les fis même river, pour qu'il ne fût pas poffible qu'elles fe lâchaffent ; jaffurai la boîte de cuivre \& les plaques qui contenoient \& aflujétifoient l'objectif, en les attachant fortement à l'extrémité fupérieure du rayon, vis-à-vis du centre de l'inftrument, avec des fils de fer qui ne permettoient pas à l'objectif le moindre jeu.

Je fis la même chofe à l'égard de la boîte du Micromètre à l'extrémité inférieure de la lunette, en uniflant l'un \&

* Il s'en trouve auffr dans piufieurs autres endroits de l'Amérique, \& notanment en Virginie. Voy, Petri Van Muffclenbroeck, Differt. de Magnete.

C c iij 


\section{$19 O$ MESURE DES TROIS PREMIERS}

l'autre à la barre qui formoit le rayon du Secteur, par des liens plus forts, \& en plus grand nombre que ceux qui avoient été précédenıment employés au même ufage. En même temps que je prenois de nouvelles précautions, je renchérifiois fur toutes les anciennes.

A l'égard des fourchettes ou fupports ZZ, qui, dès le temps de la conftruction du Secteur, m'avoient paru foibles \& d'une longueur exceffive, M. Bouguer ne m'avoit rien laiflé à faire. L'année précédente $\mathrm{r} 74 \mathrm{I}$, en obfervant feul à Tarqui, il en avoit augmenté le nombre, \& ils avoient déjà été raccourcis de moitié; en forte que la lunette étoit auffi près du corps de l'inftument que Ia largeur des règles de chan $J J J, A$ a,$B \mathrm{~b}$, \& celle de la boîte du Micromètre l'avoient pû permettre.

M. Bouguer avoit auffi ajoûté au Secteur en r 74 I une traverfe $d d$, formée d'une lame de fer plate, pofée parallèlement au limbe, \& entrelaffée entre le rayon $C D$, \& les arcsboutans ad, bd. Cette traverfe fubfiftoit, \& jen fis placer une feconde $c c$ à l'endroit où les deux parties du rayon fe joignoient. Celle-ci embraffoit la règle de chan $J J$ adoffée au rayon, \& Iui ćtoit attachée avec de fortes vis. Je liai enfuite, \& jaffermis toutes les parties du Secteur, en faifant paffer un gros fil de fer, tendu à force avec un étau à main, depuis les deux trous $\ddot{e} e$, pratiqués près du centre, jufqu'aux deux bouts $c c$ de ma nouvelłe traverfe. De là le même fil alloit s'attacher aux points extrêmes $d d$ de la traverfe inférieure; \& enfin aux points a $\& b$ de la règle de fer qui portoit le limbe. Ce fil de fer, ou pluftôt tous ces fils tendus dans le plan du limbe, rendoient l'enfemble du Secteur plus ferme \& plus infexible, fans prefque rien ajoûter à fon poids. 


\section{DEGRES DU MERIDIEN.}

'Avant que de remonter l'inftrument, je fis aufi planer le limbe, dont la furface étoit un peu convexe; \& en le remettant à fa place, j’eus foin de lui procurer une fituation bien verticale qu'il n'avoit jamais eue dans toutes nos obfervations antérieures; ce qui nous avoit toûjours obligés de tenir le cheveu fort détaché du limbe, parce que lorfquil touchoit au bord inférieur du limbe, il s'en falloit de plus d'une demi-ligne qu'il ne touchât à fon bord fupérieur.

Enfin je remontai le Secteur après avoir tracé un nouvel arc, dont la corde étoit exactement la dix-feptième partie du rayon.

\section{A R T I C L E XVIII. Continuation du même Jujet.}

Parallélifme de la Lunette au plan du Secteur. Remarques far le film à-plomb. Mouvement du Secteur dans le plan du Méridien. Inverfions alternatives de l'Inftrument.

J'Avors placé la lunette le plus parallèlement au rayon qu'il m'ivoit été poffible, par la méthode décrite dans le procès verbal rapporté (art. VI,p. I 29 ): mais on ne peut attendre de ce moyen qu'une approximation ; \& en effet, lorfque j’eus une Méridienne tracée, je reconnus par obfervation, que le plan du Secteur étant dirigé dans celui du Méridien, l'étoile paffoit au fil vertical de la lunette I I à I 2 fecondes avant l'heure de fa médiation conclue par des hauteurs correfpondantes. Pour n'affurer mieux du fait, je retournai le Secteur, en préfentant à l'occident la face du limbe, qui avoit jufque-tà regardé l'orient; \& je vis que l'étoile tardoit d'autant de 
192 MESURE DES TROIS PREMIERS

fecondes au fil vertical, qu'elle avoit avancé dans la fituation précédente. J'approchai le verre objectif du plan de l'inftrument; \& après quelque tâtonnement, qui me fit perdre plufieurs obfervations, je rćufls à faire paffer l'étoile au fil vertical à l'heure du calcul. Je n'infilte pas fur un point capital, \& qui fait la bafe de toutes les obfervations: l'attention fcrupuleufe de bien caler l'inftrument, en forte que le fil-àplomb, pendant librement du centre, rafe le limbe fans $y$ : toucher dans les deux fituations inverfes de l'inftrument. La réunion de toutes ces circonftances m'afluroit que l'axe optique de la lunette 'étoit bien parallèle au plan du Secteur. (Voy. art. $X$, pag. I so).

Nous avons manqué d'une commodité que les Obfervateurs fe font procurée depuis quelques années, fur-tout pour les grands inftrumens; je veux dire d'un fil d'argent pour furpendre le plomb. Ce fil eft prefqu'aufí fin qu'un cheveu, \& il eft plus rond \& plus égal. A fon défaut, nous nous fervions d'un fil de pite; après avoir reconnu, par expérience, qu'un filet compofé de cheveux noués bout à bout, \& chargé du poids de deux onces, étoit fujet à fe rompre fur une longueur de douze pieds. Nous ajoûtions au fil de pite un bout de cheveu à l'endroit qui répondoit vis - à - vis du limbe; mais ce cheveu tournant fur fon axe, \& fouvent inégal dans fa longueur, ne partageoit pas toûjours de la mêne manière le point qui terminoit l'arc tracé fur le limbe. Pour remédier à ce défaut, je prenois tous les jours le foin de caler l'inftument fur le point, une ou deux heures avant le pafiage de l'étoile; afin que le cheveu eût le temps de perdre fon mouvement de rotation: l'extrémité du cheveu noué avec le fil de pite débordoit 
le noud, \& formoit une efpèce d'index dont la directionz revenoit toûjours la même, lorique le fil-à-plomb \& le poids étoient dans un parfait repos; ce qui me fervoit de repaire pour leur procurer toûjours la même fituation. Je vérifiois uI moment avant l'obfervation fi l'inftrument étoit bien calé; \& auffr-tôt qu’elle étoit terminée, j’examinois de nouveau fi le cheveu répondoit au milieu du point. Quelquefois je trouvois une petite différence, caufée peut-être par l'action de la main fur la vis du Micromètre; quoique l'inftrument eût un point d'appui à fa partie inférieure, \& que j’euffe une grande attention à ne point appefantir la main en tournant l'index. Alors j'évaluois en tiers ou en quarts de la largeur du cheveu, ce qu'il s'en falloit que le point ne fût coupé en deux également, \& j’avois égard à la différence que j’avois remarquée. Le diamètre moyen des cheveux dont je me fervois m'étoit connu: je favois par l'expérience que jen avois faịte, qu'il falloit, à très-peu près, foixante largeurs d'un cheveu d'Indien pour couvrir une ćtendue de trois lignes; \& par conféquent que le cheveu dont je me fervois cachoit fur mon limbe un efpace de trois fecondes. D'ailleurs, les différences que j’avois à évaluer ne pafioient pas ordinairement une demi-largeur de cheveu: ainfi j’avois peu d'erreur à craindre dans cette correction.

Pour faire ceffer pluftôt les of cillations du fil-à-plomb , \& mieux juger quand if répondoit fur le point qui terminoit l'arc; nous avions prefque toûjours tenu, fors de nos obfervations précédentes, le poids plongé dans un vafe plein d'eau. La réfraction qui faifoit paroître ce poids plus élevé quil ne l'étoit réellement, nous avoit quelquefois fait juger quil pendoit

Dd 


\section{MESURE DES TROIS PREMIERS}

très-librement, quoiqu'en effet il touchât le fond du vale. Les cheveux en fe mouiliant font fujets à s'alonger à un tel point, quon ne fauroit être trop en garde contre cet accident. Mais un fait plus extraordinaire $\&$ bien plus propre à induire en erreur, c'eft que l'eau, quand elle a féjourné quelques jours; fe couvre quelquefois d'une pellicule grafle \& vifqueufe, qui empêche le plomb fufpendu au fil, d'ofciller en liberté; jufque-là qu'en tranfportant doucement le vafe, il m'eft arrivé de voir le poids en fuivre les mouvemens, \& s'écarter de la ligne verticale fans reprendre fon aplomb; comme s'il eût été plongé dans de la graiffe figée. J'ai foupçonné que le vafe, qui étoit de bois, ou pluftôt le vernis de Pafto dont il étoit enduit, contribuoit à cet effet fingulier. Quoi qu'il en foit, après m'être convaincu de ce fait par mes yeux, je réfolus, pour me délivrer de tout frupule, de fupprimer l'eau \& le vale, $\&$ de laiffer pendre le plomb librement en l'air ; 'en prenant d'ailleurs des mefures pour mettre le fil à l'abri du vent, qui eft un très-grand obftacle à la juftefle des obfervations, quand on ne réuffit pas à s'en garantir.

Planche IIr. On a vû dans la defcription du Secteur, que les deux vis de régie $u n$, portant fur les tenons $M M$, aux deux bouts du limbe, fervoient à le contenir dans une fituation fixe; \& qu'en preffant l'une des deux \& en lâchant l'autre, on inclinoit plus ou moins le Secteur dans le plan du limbe: je craignis donc de gêner l'inftrument en faifant agir à la fois les deux vis $n n$ fur les tenon's, comme nous avions fait dans nos premières obfervations de 1739 . Dans celles que je fis feul depuis, \& particulièrement dans celles dont il eft ici queftion, jeus toûjours fơn de lâcher Ia vis fupérieure, \& de ne 
faire ufage que de l'inférieure, fur laquelle l'inftrument repofoit par fon poids. II fuffifoit d'enfoncer cette vis, qui appuyoit fur un des tenons $M$ : elle repouffoit le limbe infenfiblement, jufquà ce que le fil-à-plomb battît fur l'extrémité de l'arc, au point $\alpha$ ou au point $\omega$, fuivant la fituation de l'inftument.

Je ne dois pas compter au nombre des précautions nouvellement prifes, lors de nos dernières obfervations, celle de ne nous être pas bornés à obferver la diflance au zénith dans deux différentes fituations du Secteur, \& de l'avoir toûjours remis une feconde fois dans $\mathfrak{f a}_{\mathrm{a}}$ première fituation, pour nous affurer que l'inftument n'avoit point varié dans le premier détour. Nous avions prefque toûjours fuivi celte pratique dans nos obfervations antérieures, comme on le peut voir par les Tables précédentes. Ce qui diftingue en ce point, les obfervations correfpondantes aux deux extrémités de l'arc, defquelles il s'agit maintenant, c'eft que nous y avons encore renchéri fur cette précaution; en retournant alternativement plufreurs fois les deux inftrumens en fens contraires, M. Bouguer de fon côté, \& moi du mien. La conformité que nous avons trouvée depuis ce temps-là, l'un \& l'autre, pendant plufieurs mois, entre nos diverfes obfervations, eft une preuve évidente que nos Secteurs ne fouffrirent, aucun ébranlement dans toutes ces différentes invęrfions: \& les dérangemens fréquens que nous avions tant de fois remarqués dans toutes nos obfervations précédentes, rendoient cette preuve bien néceffaire.

J'omets, pour éviter une exceffive longueur, le moyen d'éclairer les fils d'une manière toûjours uniforme, \& le détail de plufreurs autres attentions utiles, que j’ai eues \& que je ne vois décrites nulle part. Je me hâte de paffer au point le plus important. 


\section{A R T I C L E X IX.}

\section{Continuation du même fujet.}

Parallaxe des fils au foyer de la Lunette, differente pour divers. Objervateurs, êr variable pour le même en différens temps.

ON fait, au moins depuis le temps de M. Picard (Voy. $M e \int_{0}$ de la Terre de $M$. Picard, art. $V$ ), ce que c'eft que Ia parallaxe des fils ou des foies qui fe croifent à angles droits dans les lunettes des Quarts-de-cercle, \& autres inftumens aftronomiques. Si ces fils nie fe trouvent pas bien exactement placés au foyer de l'objectif; au lieu de les voir conme appliqués fur l'objet même, on apercevra un intervalle entre l'image de lobjet qui fe peint au foyer \& $\mathrm{x}$ le plan des fils; \& felon que l'oeil changera de fituation, cet intervalle paroitra plus. ou moins grand. C'eft-là , comme on voit, une vraie parallaxe, \& elle peut fe manifenter en deux fens différens, felon que les fils fe trouveront placés à l'égard de l'oil de l'obfelvateur; c'eft-à-dire, en deçà ou au delà de l’image. Si cette inage eft plus loin de l'oeil que les fils, l'oil en s'élevant ta verra s'élever, \& en s'abaiflant il la verra s'abaiffer; en un mot, elle paroîtla en ce cas fuivre les mouvemens de ł’cil. Le contraire aura lieu fi elle eft entre l'oeil \& les fils. Il n'eft pas befoin de figure pour concevoir que cela doit arriver ainf : un moment de réflexion fuffra au Lecteur attentif pour s'en convaincre.

Ceux qui ont manié des infrumens d'Aftronomie, du. moins ceux qui ont été dans le cas de placer des foies dans: 
une lunette, favent que le meilleur moyen dans Ia pratique pour s'affurer qu'elles font bien au foyer', c'eft d'avancer ou de reculer le réticule ou chaffis qui les porte, jufqu’à ce qu'on les voie fur l'objet, comme fr elles y étoient collées; \& cela quelque fituation qu'on donne à l'œil , foit en le portant en haut ou en bas, fỏit à droite ou à gauche : tout cecí eft connu. Mais voici quelque chofe qui, je penfe, n'avoit pas encore été remarqué. Suppolé que le fait pût être prévû par la théorie, il n'en eft pas moins vrai-femblable qu'il nous eût échappé, à M. Bouguer \& à moi, comme à tant d'autres Obfervateurs, fi la conformation de nos yeux eût été moins différente.

Pendant le cours de nos premières obfervations à Tarqui, en Décembre I 739: un jour que la lunette avoit été raccourcie en rapprochant l'objectif de l'oculaire; je repréfentois à M. Bouguer qu'il falloit que les fils du Micromètre ne fuflent pas encore bien au foyer de la lunette, puifqu'en changeant l'œil de place je voyois l'image en changer auff, \& fuivre en hauflant \& baiffant les mouvemens de mon oil ; ce que M. Verguin, qui étoit prélent, éprouvoit tout comme moi. M. Bouguer me furprit, en me répondant, que la parallaxe dont je me plaignois, fe faifoit pour lui en fens contraire; puifqu'il voyoit baifer l'image de l'objet, quand il hauffoit l'œil, \& réciproquement. Je ne me fouviens point, $\&$ je n’ai point écrit que nous ayions remarqué rien de plus fur ce fujet en 1739 .

Ce n'eft cependant pas tout. Cette parallaxe, déjä différente pour les différentes vîes, eft encore variable pour le même Obfervateur. J'ai long-temps ignoré que cette remarque, fur: Dd iij. 
laquelle je n'ai été prévenu par perfonne, eût le mérite de la nouveauté. Il falloit, pour la faire, le concours des circonflances où je me trouvois : obferver de fuite une même étoile avec unne longue lumette, dans un pays ou dans une faifon, où le temps fût fort variable d'un jour à l'autre, \& fouvent couvert, en telle forte néanmoins qu'on ne laiffât pas d'entrevoir l'étoile avec la lunette.

La première mention que je trouve de ce fait fur mon journal d'obfervations, eft du 27 Décembre I 740, quoique je m’en fuffe aperçû pluftôt. J'obfervois feul à Quito avec notre Secteur ordinaire; le Ciel étoit légèrement couvert de nuages clairs \& déliés, qui ne me déroboient pas la vûe des étoiles. Je reconnus avec la plus grande évidence, que la parallaxe des fils, qui la veille étoit très-confidérable par un temps clair \& ferein, avoit entièrement ceffé ce jour-là; en forte que l'étoile ne changeoit plus de fituation apparente, quoique mon oil changeât de place. Si c'eût été la première fois que je m’en fuffe aperçû, j’aurois pû attribuer cette différence à un changement paffager dans la difpofition de mon oil ; ce qui funt retombé dans le cas de la première remarque au fujet des deux différentes vûes: mais les preuves que j’avois déjà que cette caufe ne fuffifoit pas pour expliquer le fait, fe multiplièrent de jour en jour; \& l'année fuivante i 74 I , je m'affurai encore plus particulièrement dans le cours d'une longue Suite d'obfervations, que je fis à Quito avec une lunette de I 4 pieds, fcellée dans un mur, que la parallaxe des fils changeoit fouvent très-fenfiblement, \& non feulement du jour au lendemain, mais quelquefois d'un moment à lautre; fuivant les différens états de l'atmofphère, \& felon le plus ou le moins de lumière de l'étoile. Comme il en paffoit dans ma lunette un affez grand 
nombre de différentes grandeurs, dont quelques-unes paffoient à de courts intervalles l'une de l'autre, \& que jobfervai plufreurs mois de fuite; $\vec{j}$ eus tout le temps de bien vérifier le fait. Je remarquai conftamment que lor (que javois atteint une étoile de la cinquième ou frxième grandeur avec le fil mobile du Micromètre, elle ne me paroiffoit point fe détacher du fil, quoique je hauffafte \& baiflafle l'œil. Les fils de foie, les feuls dont nous nous fommes fervis, \& qui font beaucoup plus fins que ceux d'argent, font auff bien plus propres pour ces fortes d'obfervations.

Je remarquai encore que plus les étoiles étoient brillantes, plus leur image fe peignoit loin de mon ceil, \& au delà des fils du Micromètre; ce que je reconnoiffois, comme je l'ai déjà dit, parce qu'en hauffant \& baiffant l'œil, cette image paroiffoit en fuivre les mouvemens. C'eft fans doute par la même raifon que je ne remarquois point ordinairement de parallaxe fenfible, même à l'égard des étoiles de la feconde grandeur, quand elles pafioient de jour dans la lunette.

Je communiquai dans le temps à $M$. Bonguer ces différentes remarques, à mefure que je les faifois; je n'ai point fù s'il les avoit faites de fon côté: mais je fuis fi fur de ce que j’ai vî, que je ne puis douter qu'il n'ait vû les mêmes choles que moi.

Je réfume les faits que je viens d'expofer, \& j’en tire lęs conféquences immédiates. M. Bouguer voyoit quelquefois limage de l'étoile en-deçà des fils du Micromètre, à la même heure où je la voyois au delà. Donic nous voyions alors lui $\&$ moi, deux images différentes; ce qui fuppole dans l'objectif un: changement de foyer relatif aux différentes vîes. L'Obfervateus. 


\section{MESURE DES TROIS PREMIERS}

Prefbyte aperçoit celle des deux images qui eft la plus éloignée de fon œil, \& la plus voifme de l'objectif; le Myope a des apparences toutes oppofées. Si donc il n'y avoit point d'oculaire, la lunette feroit plus courte pour le prefbyte, \& plus longue pour le myope. Cependant la théorie nous enfeigne que l'Obfervateur myope ne peut voir diftinctement l'image peinte au foyer de la lunette, fans approcher l'oculaire de cette image, pour augmenter la divergence des rayons, qui, fans cette précaution, réunis trop tôt dans fon œil, y rendroient la vifion confufe: \& que le prefbyte au contraire doit éloigner l'oculaire de l'inage, pour rendre les rayons plus convergens; \&. hâter leur réunion fur la rétine. $\mathrm{Or}$ on ne peut approcher l'oculaire du foyer de la lunette fans la raccourcir', ni l'éloigner du même foyer fans la ralonger. La lınette garnie d'un oculaire doit donc être plus courte pour le myope \& plus longue pour le prefbyte : \& l'expérience y eft conforme.

Ainfi donc, la lunette s'accourcit pour le myope du côté de l'objectif, tandis qu'elle s'alonge.du côté de l'oculaire: \& réciproquement pour le prefbyte. Or ces deux variations relatives aux deux différentes vûes, croiffent en fens contraire avec Ia longueur des lunettes, mais la première dans un bien plus grand rapport que la feconde*. D'où il s'enfuit, que fi une lunette de grandeur ordinaire doit être raccourcie pour une vûe bafle, le contraire peut \& doit arriver dans une fort longue lunette. J'avoue que je n'en ai pas fait l'expérience.

Quant à ma dernière remarque fur la diverfité de parallaxe des fils pour un même Obfervateur en différens temps;

* Tandis que l'une croît comme la longueur du foyer de l'objectif, l'autre ne croît qu'en raifon fous-doublée, comme la longueur du foyer de l'oculaire.

jignore 
j̈ignore de quelle manière ces différences fe font manifeftées à M. Bouguer. Comme je n'ai point eu de communication de ce qu'il a lû fur ce fujet à l'Académie en mon abfence; $\&$ que j’ai crû devoir me priver de la lecture de fon livre, jufqu'à ce que le mien fût publié, je dois m’en tenir à ce que j’ai reconnu par ma feule expérience. Premièrement, je n'ai aperçû, comme je l'ai dit, aucune parallaxe fenfible dans les fils du Micromètre, lorfque le Ciel étoit légèrement couvert de petits nuages tranfparens : en fecond lieu, lorfque le temps étoit clair, \& les étoiles brillantes, j’ai toûjours vû l'image de l'étoile au dełà des fils; puifqu'elle m'a toûjours paru fuivre les mouvemens de mon œil. Enfin quoique jaie fouvent raccourci la lunette confidérablement, je n’ai jamais vû l'image en deçà deś fils, ni la parallaxe en fens contraire au mouvement de l'œil, comme M. Bouguer l'a vûe quelquefois; mais ce que j'ai vî fuffit, pour en conclurre que le même Obfervateur ne voit pas toûjours Ia même image, \& que le foyer de la lunette varie fuivant les différens états de l'atmofphère, les différens milieux que traverfent les rayons, \& le plus ou moins de lumière de l'objet.

Ce n'eft pas une chofe nouvelle que Ia multiplicité des images qui fe peignent au foyer d'une lunette: il y a longtemps qu’il eft démontré en Dioptrique, qu'un objectif, doni la courbure eft fphérique, ne réunit pas les rayons en un point; \& que plus Ia fphère fur laquelle le verre a été travaillé eft d'un grand rayon, le nombre de degrés étant fuppofé le même, plus le foyer occupe un efpace confidérable en tout fens. L'expérience confurme ici pleinement la théorie. 
Si on reçoit à traver's un objectif de is à i 6 pieds de foyer fur un papier banc \& dans une chambre obfure, l'image d'un objet éclairé, on reconnoîtra que le lieu où cette image fe projette diftinctement n'elt pas un plan mathématique, \& qu'on peut éloigner ou approcher un peu le papier de lobjectif, fans que l'image foit confufe. On fera même embarraffé à déterminer le point précis où elle eft la plus diftincte.

Mais une autre raifon, long-temps ignorée, contribue au même effet, \& beaucoup plus puiffamment que la précédente: c'eft la différente nature des rayons de lumière, découverte dûe, ainfı que tant d'autres, à $M$. Newton. Ce Philolophe a fait voir que les rayons, en traverfant une même furface, fe rompent fous différens angles; \& que felon leurs divers degrés de réfrangibilité, ils fe réuniffent, à des diftances inégales, en différens foyers, où ils forment autant d'images diverfement colorées. Cette expérience eft connue de tout le monde; mais il étoit naturel de penfer que c'étoit l'image la plus lumineufe, celle qui occupoit le milieu de la profondeur du foyer, celle enfin qui répondoit au point le plus éclairé, qui étoit toûjours aperçûe par les différens Obfervateurs, \& fur-tout par le même : au lieu qu'il eft déformais prouvé \& confirmé par l'expérience, que le même jour \& dảns le même inftant, deux Obfervateurs voient dans la même lunette deux images différentes; \& que le même Obfervateur, en différens jours \& à différentes heures, ne voit pas toûjours la même image.

Avant la découverte de la diverfe réfrangibilité des rayons de lumière, Defcartes, \& plufieurs autres Phyficiens après lui, avoient cherché les moyens de donner aux objectifs une figure différente de la fphérique, \& propre à procurer la réunion 
des rayons en un point. C'eft prefque à ce feul but qu'ont tendu tous leurs efforts, comme à l'unique moyen de perfectionner les lunettes; jufqu'à ce qu'il ait été prouvé qu'on ne. remédieroit par-là quà la moindre partie de la diffufion du foyer; puifque celle qui eft caufée par la diverfe réfrangibilité des rayons de lumière eft incomparablement plus grande que celle qui eft produite par la fphéricité du verre*. Depuis ce temps, on a prefque regardé comme fans remède l'imper- de 5449 a a $V_{0 y}$. Compleat fection des lunettes, \& M. Nowton même parut avoir aban- ${ }_{b y} y$, of oprticks donné quelques idées qu'il avoit eues pour corriger le défaut n.0340.

de la courbure fphérique : du moins il tourna fes vûes vers les Télefcopes catoptriques, dont il a tiré un fi grand parti. Les chofes en étoient demeurées là, lorfque le favant M. Euler, * Mćmoires de confidérant que les rayons qui entrent dans l'aily fouffrent quatre l'Acadénie de réfractions, en a conclu qu'il doit être. poffble d'arranger telle-page 279 .

ment quatre furfaces réfringentes, que les foyers de toutes. fortes de rayons convinffent dans un feul point, à quelque diftance que fe trouvât l'objet. Partant de ce principe, il a réfolu le problème; en donnant les dimenfions d'un objectif tel, que les rayons les plus dịverfement réfrangibles, après avoir traverfé une lentille d'eau contenue entre deux verres menifques femblables, de courbures fphériques, \& après avoir fouffert quatre réfractions, fe réuniffent en un feul point.

Je reviens à mon fujet. Parmi une foule d'images, placées fur l'axe optique d'une lunette ordinaire; à différentes diftances de fon objectif; la plus forte, la plus lumineufe, celle en un mot qui occupe le milieu de l'efpace qui les renferme toutes, comment n'eft-elle pas toûjours la première, \&.même la feule aperçûe, foit par différens Obfervateurs, foit par le Ee ij 


\section{MESURE DES TROIS PREMIERS}

même? Effayons de répondre à cette difficulté.

Quoique, géométriquement parlant, l'image qui occupe le centre du foyer doive être la plus vive \& la plus brillante de toutes; cependant comme la dégradation de lumière d'une image à l'autre fe fait par degrés infenfibles, il y a autour du foyer phyfique, un certain efpace, dans lequel toutes les images font prefque également propres à être aperçûes. Suppolons que cet efpace occupe un pouce, ou la cinquième partie de la profondeur du foyer fur l'axe d'une lunette de douze pieds, dans laquelle les foyers des différens rayons sétendent fur une longueur de plus de cinq pouces, comme il fuit de ce * Mćmoires de que M. Newton, \& tout récemment M. Euler*, ont démontré. l'Acadénie de Berlin, 1747 , page $2>6$.

Entre toutes les images prefque également propres à être aperçûes, fur cette longueur fuppofée d'un pouce; chaque Obfervateur doit voir plus diftinctement, à l'aide de l'oculaire qui fert ì les groffir, celle qui fe trouve fituée à la diftance la plus convezable à la conformation de fes yeux: ainfi, celui qui a la vûe baffe verra une image plus voif́ne de fon oil ; \& celui qui a la vûe longue verra une image plus éloignée. Et fi le chaffis qui porte les foies fe trouve placé entre ces deux images, la paralJaxe des fils aura lieu néceffairement en fens contraire pour les deux Obfervateurs; comme cela nous eft effectivement arrivé à $M$. Bouguer \& à moi.

It refteroit à expliquer pourquoi l'image de l'étoile, laquelle d'un temps clair \& ferein me paroiffoit toûjours au delà des fils du Micromètre, venoit fe placer fur ces fils dans un temps légèrement couvert; \& par conféquent pourquoi le foyer de l'objectif fembloit s'alonger pour moi en ce dernier cas. Je me contenterai de faire fur "cela les remarques fuivantes. 
Prémièrement, de ce que je voyois prefque toûjours l'image au delà des fils du Micromètre, il femble qu'on pourroit inférer que la lunette étoit trop courte pour ma vâe : cependant cette conféquence eft difficile à concilier avec deux faits certains. L'un, que j’avois tâché de donner à la lunette avec laquelle j’ai fait ces expériences, la longueur la plus convenable à mes yeux; en l'effayant fur plufieurs étoiles avant que de la faire fceller: l'autre, que depuis qu'elle étoit fixée, je l'avois raccourcie peu à peu, de plus d'un pouce, fans avoir pû faire ceffer la parallaxe, qui me faifoit toûjours voir l'étoile au delà des fils, quand le Ciel étoit clair.

Secondement, puifque cette paraliaxe, que je-n'avois pît anéantir en raccourciflant la lunette, devenoit nulle, ou tout-àfait infenfible par un temps légèrement couvert; il faut bien que I’image, qui d'un temps ferein fe peignoit au delà des fils, vînt, dans la nouvelle difpofition de l'atmofphère, fe projeter fur leur plan même. Elle s'approchoit donc alors de mon œil, \& s'éloignoit de l'objectif: ce qui femble prouver que, dans le premier cas, l’image étoit formée par les rayons le pluftôt réunis, \& dans le fecond par ceux du plus long foyer. Mais quelle pouvoit être la çaufe qui affoibliffoit ou interceptoit alternativement, tantôt certains rayons, \& tantôt d'autres, pour ne laiffer voir au même fpectateur que l'une ou l'autre image? L'expérience prouve que les rayons rouges font ceux qui ont Ie plus de facilité à pénétrer l'atmofphère. Le Soleil \& les autres aftres, vîs près de l'horizon quand l'air eft chargé de ,vapeurs, paroiffent teints de cette couleur. Les layons rouges. font auff les moins réfrangibles, \& par cette raifon ce font ceux qui fe réuniffent le plus loin du verre on ils font rompus. 
Ceci quadre à quelques circonftances des apparences optiquès que jai remarquées, mais non à toutes. On pourroit fuppofer que lorfqu'il n'y avoit point de parallaxe, les feuls rayons rouges avoient la force de percer les petits nuages dont le Ciel étoit alors couvert ; \& que leur foyer étant plus long, l'image atteignoit, en ce cas, le plan des fils qu'elle ne pouvoit atteindre quand ces rayons ne dominoient pas fur les autres. Mais il femble auffr que par la même raifon, l'image de l'étoile auroit dû alors paroître rougeâtre, comme il arrive aux aftres près de l'horizon, \& c'eft ce que je n'ai pas remarqué.

Je craindrois de trop donner à la conjecture, en entrant dans un plus grand détail, \& je laiffe à M. Bouguer le foin d’approfondir une matière, fur laquelle il a fur moi l'avantage d'avoir publié il y a plus de vingt ans* de favantes recherches. Il a encore, dans l'occafion préfente, celui de pouvoir fonder fes raifonnemens fur un plus grand nombre de faits que moi; outre fes propres expériences, je lui ai communiqué toutes les miennes. Pour moi, je fais feulement que M. Bouguer a quelquefois vû la parallaxe des fils du Micromètre, dans un fens contraire à cefui où nous la voyions, M. Verguin \& moi. Du refte je ne fais, au fujet de ces apparences optiques, que ce que j’en ai.vî par mes propres yeux, dans mes oblervations particulières. Je viens au point le plus effentiel, \& par lequel je terminerai cette longue differtation fur les nouvelles précautions prifes dans mes dernières obfervations. C'eft le moyen que j’ai employé pour éviter l'erreur de la parallaxe des fuls du Micromètre, qui jufque-là nous avoit été fi fatale.

* Effá fur la gradation de la Lumière, Paris, 17290 


\section{A R T I C L E X X.}

\section{Continuation du mêtme fujet.}

De la manière d'éviter la Parallaxe des fils au foyer de la Lunette.

Da s s toutes les obfervations qui ont précédé les fimultanées, nous n'avions pris, contre la parallaxe des fils, d'autres précautions que celle qui eft indiquée dan's le procès verbal des anciennes obfervations de 1739 à Tarqui (art. VI, page I 33), \& dans la Table des obfervations faites par $M$. Bouguer au niême lieu en 174 I ( art. $X V$, page 179 , après le $5^{\text {me }}$ Ré(ultat). Cette précaution confiftoit à placer toûjours l'œil au même point; \& pour y réulfir plus fûrement, nous appliquions au devant de l'oculaire un bout de tuyau de carton, percé d'une très-petite ouverture, qui fervoit de pinnule. Cet expédient remédieroit à tout $f_{1}$ la parallaxe des fils n'étoit pas variable: car fuppofant que la pinnule fût fituée obliquement à l'égard de l'axe de la lunette; il eft bien vrai que l'œil placé à la pinnule, rapporteroit l'image à un point du réticule, autre que celui où il la rapporteroit s'il la voyoit d'un point de l'axe même; \& que par conféquent la hauteur de l'aftre lui paroîtroit augmentée ou diminuée : mais ce feroit d'une quantité toûjours égale \& du même fens, tant que l'inftrument refteroit dans la même fituation; \& lorfqu'on le retourneroit, l'erreur feroit encore la même en fens contraire. Ainfi cette erreur fe confondroit avec celle qui réfulte du défaut de parallélifme de la lunette au rayon d'où on commence à compter les angles: ces deux ejreurs n'en feroient qu'une, \& la fomme des deux 
feroit reconnue par l'opération ordinaire du renverfement, quå fert à vérifier la pofition de la lunette. Mais puifqu'il eft certain que la parallaxe des fils eft variable, \& que la diftance de l'objectif à l'image qui fe peint à fon foyer n'eft pas toûjours la même, il s'enfuit que la précaution de la pinnule fixe n'eft pas fuffrante; \& il eft évident, par les loix de la projection, que Pœil fitué obliquement par rapport ì l'axe de Ia lunette, ne peut manquer, quoiqu'immobile, de rapporter à divers points du réticule, les différentes images qu'il voit en effet plus ou moins éloigncées, en divers jours \& à diverfes heures.

On peut concevoir les images qui fe peignent aux différens foyers de l'objectif, comme autant de tableaux rangés les uns au devant des autres le long de l'axe optique de la lunette: elles fe préfentent donc, à un œil fitué obliquement, fous le même point de vîe que feroient les décorations à couliffe d'un côté du théatre: d'où il s'enfuit que parmi toutes ces images, l'œil doit rapporter celle qui fera vifible pour lui, à différens points du réticule, felon quelle fera plus voifine ou plus éloignée. II n'y a qu'une feule pofition, où l'œil puiffe éviter cette erreur : ćêt celle où il feroit placé dans l'alignement même des centres de toutes les images; c'eft-à-dire; dans l'axe optique de la lunette. Alors tout cet axe fe projeteroit fur un feul point du réticule, ou du plan des fils du Micromètre; \& à quelque diftance de l'oeil que l'image funt tranfportée par la variation \&u foyer, le centre de cette

- image répondroit toûjours, fur le plan des fils, au point où ce plan eft rencontré par l'axe.

I eft vrai que fi l'image eft étendue, if n'y aura que fon point 
point central qui fera exempt de parallaxe; \& que les bords de l'image en fouffriront une plus ou moins grande, felon que l'image occupera plus ou moins d'efpace. Et par une conféquence néceflaire, fi le réticule fe trouve placé, comme il l'eft ordinairement, entre les deux foyers extrêmes des rayons inégalement réfrangibles, il arrivera que dans la même lunette, l'oil myope, placé en $O$, projetant \& mefurant fur le réticule $A B$, l’image $D G$ qu'il voit en deçà, la jugera plus grande qu'elle n'eft en effet, \& la fera égale à $\Delta \Gamma$; tandis que l'oil prefbyte, rapportant fur le même plan $A B$ l'inage $d g$ qu'il voit au delà, la jugera plus petite; \& la mefurant entre les fils, la trouvera égale à $\delta \gamma$.

C'eft vrai-femblablement pour cette raifon, que lorfque nous examinions èn r 739 la valeur des parties du Micromètre fur une longueur de $8 \mathrm{o}$ pieds (part. II, art. 111 , page 1 I 3), dont l'image occupoit environ $4 \frac{1}{2}$ lig. au foyer de la funette, \& foutendoit.un angle de près de 9 minutes, $M$. Bouguer trouva cette image égale à I 196 parties du Micromètre; au lieu que je n'en trouvois que 1 1 93. Cette différence, qui n'eft que de $\frac{x}{399^{\circ}}$ partie, \& qui fe trouva alors d'une feconde, devient tout-à-fait infenfible fur de plus petites quantités; mais fût-elle beaucoup plus grande, elle ne tireroit nullement à conféquence dans le cas préfent, où il eft queftion d'une étoile qui n'occupe qu'un point dans la lunette. Il eft donc certain que fi l'oil eft placé dans l'axe optique, à quelque diftance de l'oil que fe peigne l'image de l'étoile, elle fera toûjours vûe au même point du réticule; au lieu que fi l'œil voit l'axe obliquement, c'eft en vain qu'on rend fixe \& immobile la pinnule où il eft placé : il fuffit que l'image change de lieu fur l'axe en différens temps, 


\section{MESURE DES TROIS PREMIERS}

pour qu'il la voie changer de lieu fur le réticule, \& s'approcher ou s'éloigner de l'interfection des fils.

Quelque évidemment que cette conclufion fe déduife de mes propres expériences fur les variations de longueur du foyer de lobjectif, j’avone qu'elle ne s'étoit pas préfentée bien nettement à mon efprit, jufqu'au temps des demières obfervations que je fis feul à Tarqui à la fin de $1742 ; j ’ y$ fus alors conduit comme par degrés. Je vais rendre compte des circonftances qui concoururent à m'ćclairer, \& qui me frent enfun trouver un remède à la parallaxe des fils, plus efficace que ceux que nous avions employés jufqu'alors. C'eft par ce détail que j’acheverai d'informer le Lecteur, des précautions nouvelles que je pris dans mes dernières obfervations à Tarqui.

Après avoir recomnu, comme je l'ai dit, que l'inftument avoit fouffert quelque altération dans fon tranfport de Quito à Tarqui; après l'avoir démonté, raffermi, reconftuit, y avoir fait toutes les réparations \& nouvelles additions dont j’ai parlć, avoir épuifé toutes les précautions rapportées dans les articles XVII, XVIII, X IX \& XX; enfin après avoir domé à la lunette du Secteur une longueur telle que je n'apercevois dans les fils aucune parallaxe à l'égard de l'étoile, quand le brouillard étoit tranfparent, ce qui faifoit communément les plus beaux jours de Tarqui : jeflayai de rendre la parallaxe infenfible dans tous les cas, en rétréciffant l'ouverture de l'objectif de la lunette, par le moyen de plufieurs diaphragmes de carton inćgalement ouverts, que jy appliquois alternativement, \& que je fubftituois l'un à l'autre, fuivant que le Ciel étoit plus ou moins pur. Je réuffis par ce moyen à diminuer la grande fcintillation de l'étoile, qui me parut mieux terminće. La paral- 
laxe des fils étoit moindre auffr ; mais elle fubfiftoit encore, $\&$ toûjours du même fens : c'eft-à-dire, qu'en haufant l'œil, je voyois toûjours l'étoile s'élever, \& en l'abaiffant, s'abaiffer à l'égard du fil horizontal.

J'eus recours à ırotre expédient ordinaire : j’adaptai au devant de l'oculaire un bout de tuyau de carton percé d'un trèspetit trou. Comme j'étois feul à obferver, il me fut facile de rendre cette pinnule fixe, aufi-bien que l'oculaire même; ce qui n'eft pas poffible quand deux Obfervateurs, dont la vûe eft inégale, obfervent enfemble: puifqu'il faut alors alternativement approcher l'oculaire du foyer de l'objectif pour l'un, \& l'en éloigner pour l'autre. La pinnule une fois fixée, j'étois bien für que mon œil étoit toûjours placé au même point; mais je ne tardai pas à m'apercevoir que mes obfervations n'en étoient pas pour cela plus uniformes, ou pluftôt qu'elles ne l'étoient pas toûjours. J'obfervois quelquefois pendant deux ou trois jours la même diftance au zénith, à trèspeu près; je trouvois enfuite d'un jour à l'autre des différences de 6 ày fecondes, ou plus; quoique je n'euffe touché à linftrument dans l'intervalle, que pour faire répondre le filà-plomb très-exactement au même point : \& c'étoit lorfque le Ciel étoit chir \& que les étoiles étoient brillantes, que je remarquois les plus grandes différences.

Jamais je n'avois pû m'accoûtumer à regarder comme inévitables des variations auff confidérables, \& moins encore de plus grandes, que nous avions quelquefois éprouvées du jour au lendemain, dans le temps que nous obfervions enfemble ă Tarqui. M. Bougutuer \& moi, en I 739 . Je lui avois dèsJors témoignéé ma furprife, de voir qu'un inftrument de douze 

pieds de rayon nous donnât quelquefois des obfervations moins conformes entr'elles que n'eût fait un Quart-de-cercle de trois pieds, placé dans un lieu commode. M. Bouguter, dans le temps dont je parle, paroiffoit perfuadé qu'il n'étoit pas ponfible de parvenir à une plus grande exactitude; je lui avois expofé fur tout cela mes doutes \& mes fcrupules : il ne les avoit pas fait ceffer entièrement, mais ils avoient été fufpendus par fa préfence, par le concours de fes lumières, \& le poids de fon témoignage.

Privé de ces fecours, lorfque j’allai répéter feul à Tarqui nos anciennes obfervations en $174^{2}$, je fentis renaitre toutes mes inquiétudes; \& je rélolus de ne pas terminer mon travail, que je ne fuffe à quoi m'en tenir fur ces variations fubites fi étranges, \& qui me paroifioient toûjours fi incompatibles avec la grandeur de notre infrument.

Je me rendois à mon obfervatoire quelque temps avant I'heure de la médiation d'є d'Orion; \& je me préparois à cette obfervation par celle de plufieurs autres étoiles, qui paffoient dans la lunette quelques minutes auparavant. Une nuit que Ia lumière des étoiles étoit fort vive, je trouvai, en plaçant le curfeur du Micromètre fur une de celles qui précédoient $O$ rion; un nombre de parities affez différent de celui auquel je m’attendois en conféquence de mes obfervations précédentes. Je foupçonnai que le tuyau de carton qui portoit la pimnule s'étoit dérangé : jôtai tout cet attirail; \& au lieu d'aller, avec le fil mobile horizontal, à la rencontre de l'étorile $\varepsilon$ qni alloit paffer, ơ de la fuivre avec ce même fil, comme nous l'avions toûjours pratiqué; je plaçai d'avance l'index du Micromètre, fur le nombre de parties que javois obfervées plufieurs fois, les nuits où if n’y avoit point de parallaxe, \& $j$ attendis enfuite que l'étoile 
vint fe placer fur le fil ainfi difpofé. Mais comme il n'y avoit plus de pinnule, je m’aperçûs, auffr-tôt que l'étoile fut entrée dans la lunette, que la parallaxe étoit fi grande, qu'en hauffant \& baiffant l'œil, je tranfportois à mon gré l'étoile au deffus ou au deffous du fil, à une diftance de part \& d'autre plus que double de fon diamètre. Je me hâtai de replacer la pinnule, \& de l'arrêter, au point d'où mon seil voyoit l'étoile fuivre la route que je lui avois; pour ainf dire, tracée, par la pofition que j’avois donnée d'avance au fil mobile. J'eus foin d'affermir la pinnule en cet état, \& j'apportai une grande attention à ne la plus déranger. Depuis ce temps, quoique je repriffe notre pratique ordinaire, de ne pas laiffer le fil mobile au point où je l'avois conduit la veille, mais de l'amener à chaque fois fur l'étoile, en comptant les parties qui mefuroient fa diftance au fil fixe; j’en retrouvai toûjours, à trèspeu près, le même nombre; \& je ne remarquai plus, dans les obfervations faites à peu de jours d'intervalle, que ces petites différences, qui fe peuvent attribuer à la difficuité de bien juger fi le fil horizontal partage l'étoile en deux parties égales, fi le fil-à-plomb coupe bien également le point qui termine l'arc; ou à quelqu'autre caufe femblable.

Par tout ce qui a été expliqué précédemment, on doit voir que je m'étois garanti de l'erreur de la parallaxe, en fuivant le procédé que je viens de décrire; dont l'effet étoit dè me faire voir l'étoile toûjours au même point du réticule, foit qu'il n'y eût pas effectivement de parallaxe, comme lorfque l'image de l'ćtoile venoit fe peindre fur le plan des fils, foit qu'il y en eût, comme les nuits où l'image n'atteignant pas le plan des fils, reftoit au delà, \& fembloit obéir aux mouvemens 
de mon œil. Or je ne pouvois voir l'image répondre au même point du réticule, quoiqu'elle fût tantôt plus proche, \& tantôt plus loin de moi, quautant que mon oil étoit dans la ligne qui joignoit les centres des diverfes images; c'eft-à-dire, qu'autant qu'it étoit dans la direction de plufieurs points de l'axe, ou dans le prolongement de l'axe même, \& par conféquent à l'abri des erreurs de la parallaxe que je cherchois à éviter.

M. Bouguer, à qui je mandai que je croyois avoir un moyen de fauver la parallaxe, ne me répondit rien fur cela; fans doute il s'en étoit garanti, ou de la même manière que moi, ou par quelque moyen ćquivalent, \& peut-être meilleur. D'ailleurs, comme dans les obfervations correfpondantes aux miennes, qu'il faifoit alors à Cotchefqui, il fe fervoit d'un Secteur \& d'une lunette de 8 pieds, \& que la parallaxe dont il eft ici queftion crô̂t en même raifon que la longueur du foyer de l'objectif; M. Bouguer n'avoit à craindre avec fa lunette de 8 pieds, qu'une erreur qui n'étoit pas tout-à-fait les deux tiers* de cefle à laquelle j'étois expofé avec une lumette de I 2 pieds; \& c'eft ce qui a pû contribuer à le déterminer à employer un Secteur d'un plus court rayon. Au refte, fi M. Bouguer a employé le même expédient que moi, je protefte que je n’en ai, jurfqu'à ce moment, aucune connoiflance. J'ai raconté tout fimplement, comment mes différentes tentatives mont conduit

* La parallaxe eft ici l'effet d'une variacion paffagère dans le foyer de robjectif. Cette variation croît proportionnellement à la longueur de ce foyer, laquelle elt prife communément pour la longueur même de la lunette; quoiqu’à parler rigoureufement cette longueur foit égale à la fonme des foyers des deux verres, $\&$ que le foyer de l'oculaire croiffe, comne on l'a déjà remarqué, en moindre raifon que celui de l'objectif. De-là il s'enfuit que, dans une dunetze d'un tiers plus longue, la parallaxe croît d'un peu plas d'un tiers. 


\section{DEGRÉS DU MERIDIEN.}

ì un procédé qui m’a réuffr. Il eft aifé de voir que j’aurois pû l'expofer d'une manière plus propre à le faire valoir', \& à en relever le mérite.

Le Lecteur me pardonnera, en faveur des motifs que j’ai allégués, d'avoir tant infifté, contre ma première intention, fur les préparatifs de mes dernières obfervations. Je paffe à ceș obfervations mêmes.

\section{A R T I C L E X X I.}

Demières obfervations, faites à Tarqui, au Sud de la Méridienne, correfpondantes à celles qui ont été faites en même temps, à l'extrémité Nord.

TAB LE des diffances de l'E'toile $\varepsilon$ d'Orion all Zénith de Tarqui, que j'ai objervées en $I 742$ \& 1743 , réduites au premier Janvier $I 743$.

Premiere Suite d'Olfervations,

Faites fur un arc de $3^{\text {¿ } 22^{\prime}} 15^{\prime \prime}$, dont la corde étoit égale à la $17^{\text {me }}$ partie du rayon.

\begin{tabular}{|c|c|c|c|c|c|c|c|c|}
\hline $\begin{array}{c}\text { Situation } \\
\text { du } \\
\text { Secteur. }\end{array}$ & $\begin{array}{c}\text { D А т } \\
\text { des } \\
\text { Obfervations. }\end{array}$ & $\begin{array}{l}\text { Quantite's } \\
\text { obfervées avec } \\
\text { le Micromètre. }\end{array}$ & $\begin{array}{l}\text { E'QUA } \\
\text { Préceffion } \\
\text { des } \\
\text { Ëquinoxes. }\end{array}$ & $\left|\begin{array}{c}\text { Aberration } \\
\text { de la } \\
\text { Lumière. }\end{array}\right|$ & $\begin{array}{l}\text { Nuration } \\
\text { de litAxe } \\
\text { terreltre. }\end{array}$ & $\begin{array}{c}\text { Obfervations } \\
\text { réduites au I } \\
\text { Janv. I } 743 .\end{array}$ & $\begin{array}{c}\text { QUANTITE'S } \\
\text { moyennes. }\end{array}$ & RESULTATS, \\
\hline $\begin{array}{l}\text { Le limbe } \\
\text { vers } \\
\text { l'orient. }\end{array}$ & $\begin{array}{r}1742.29 \text { Nov. } \\
30 \\
\text { I Déc. }\end{array}$ & $\begin{array}{l}+47^{\prime \prime}, 5 \\
+49,5 \\
+\quad 47,5\end{array}$ & $\begin{array}{l}+0^{\prime \prime,} 3 \\
+0,2 \\
+0,2\end{array}$ & $\left|\begin{array}{r}4^{\prime \prime}, 8 \\
-4,7 \\
-4,6\end{array}\right|$ & $\begin{array}{l}+0,1 \\
+0,1 \\
+0,1\end{array}$ & $\begin{array}{l}+43^{\prime \prime}, 1 \\
+44,9 \\
+43,2\end{array}$ & 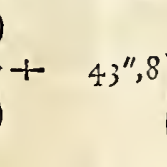 & \\
\hline $\begin{array}{c}\text { vers } \\
\text { Poccident. }\end{array}$ & $\begin{array}{l}2 \\
3\end{array}$ & $\begin{array}{l}-30,6 \\
-\quad 35,1\end{array}$ & $\begin{array}{r}+0,2 \\
+0,2\end{array}$ & $\begin{array}{r}4,4 \\
-4,3\end{array}$ & $\begin{array}{l}+0,1 \\
+0,1\end{array}$ & $\begin{array}{l}-34,8 \\
-\quad 39,1\end{array}$ & 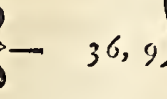 & \\
\hline
\end{tabular}

Arc du Secteur . ..... 322 15:

Double diftance au Zénith, obfervée. . . . . . $3^{\mathrm{d}} 22_{2}^{\prime} \mathrm{r}^{\prime \prime}, 9$ Premier Réfultat. Diflance apparente d'E d'Orion au Zénith de Tarqui, du côté du Nord, 


\section{MESURE DES TROIS PREMIERS}

\section{Seconde Suite d'Obfervations,}

Faites, conme les précédentes, fur un arc de $3^{\mathrm{d}} 15^{\prime 2} 22^{\prime \prime}$, dont la corde étoit égale à la $I 7^{\text {me }}$ partie du rayon.

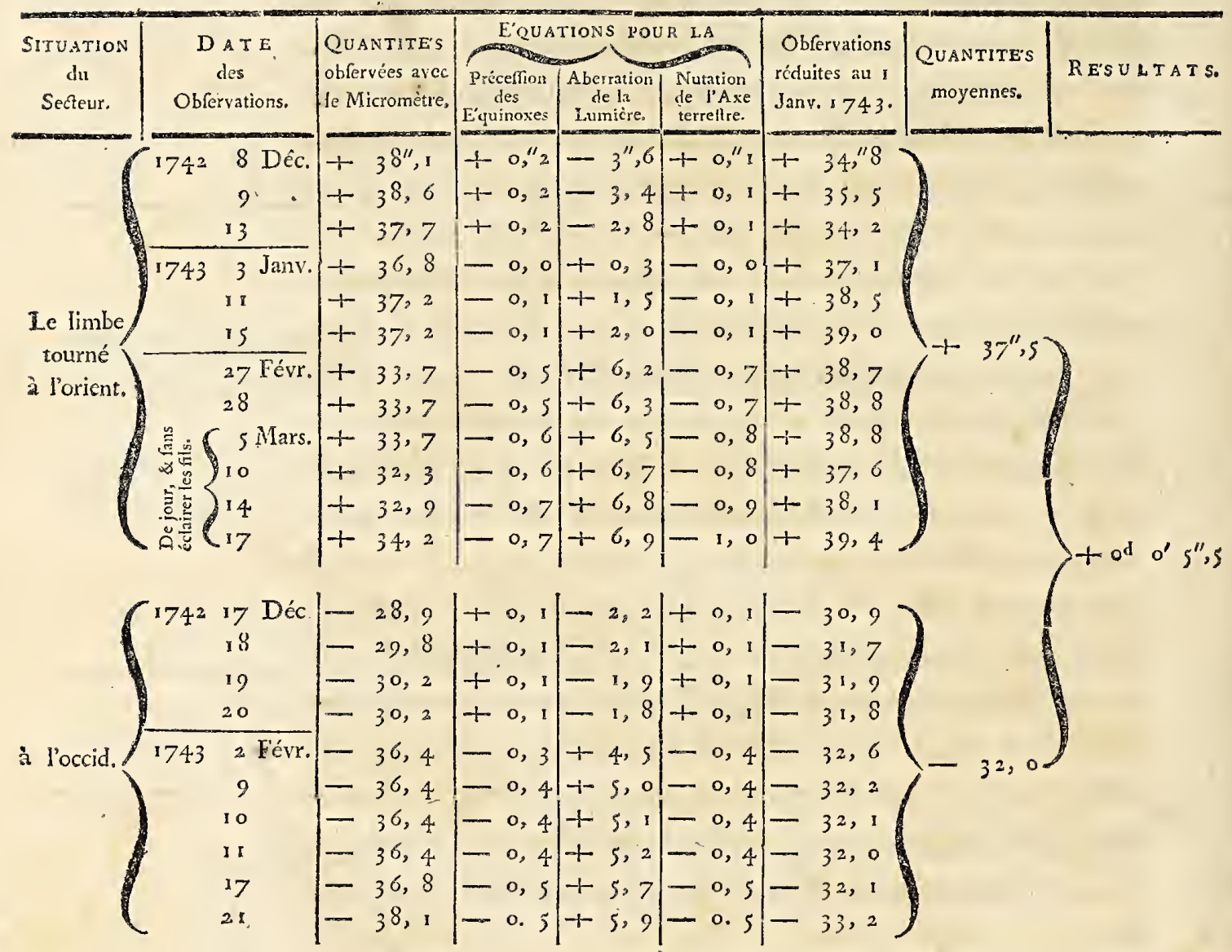

Arc du Sectetir............... 32215 ,

Double diftance au zénith, obfervée ........... $3^{\mathrm{d}} 22^{\prime} 2 \mathrm{o}^{\prime \prime}, 5$

Second \& dernier Réfultat. Di̊nance apparente de $\varepsilon$ d'Orion au Zénith de Tarqui, du côté

du Nord, réduite au a đanvier $1743 \ldots \ldots$ I $4110,2 \frac{1}{4}$ 


\section{Remarques fur les objervations de la Table précédente.}

J'étois à Tarqui dès le 20 Septembre I 742 , \& Ia première obfervation rapportée dans la Talle précédente n'elt que du 29 Novembre; ainf il fe pafia plus de deux mois avant que je pûfre avoir des obfervations fuivies. J'ai déjà parlé ailleurs des raifons qui m'en avoient empêché : l'inftrument avoit Introduction
hiforique, Sept. fouffert dans le tranfport de Quito à Tarqui; il me fallut faire venir des ouvriers de Cuenca, démonter le Secteur, en raffermir toutes les parties, y faire plufieurs, changemens \& de nouvelles réparations, le reconftruire, le remonter, mefurer le rayon, tracer un nouvel arc, dont la corde fut bien exactement partie aliquote du rayon, perfectionner à plufreurs reprifes le parallélifme de la lunette : tout cela me fit perdre un bon nombre d'obfervations, les précédentes devenant inutiles chaque fois que j'étois obligé de retoucher à l'objectif. La proximité du. Soleil aú zénith ne mavoit pas permis d'abord de tracer une Méridienne exacte: les tremblemens de terre, les arrêts fréquens de ma pendule, me caufèrent enfuite de nouveaux obftacles, dont le plus grand étoit le Ciel de Tarqui, prefque toûjours contraire aux obfervations aftronomiques. Nous ne l'avions que trop éprouvé en I 739, \& M. Bouguer en particulier en $\mathrm{r} 74 \mathrm{I}$, comme on en peut juger par les inter- vage 130 . valles de fes obfervations (Voy. la Table de l'art. $X V, p, I>8$ \& 179$)$. Enfin les différentes tentatives pour diminuer \& pour anéantir l'effet de la parallaxe des fils du Micromètre, avant que j’euffe trouvé le dernier expédient, dont j’ai donné le détail, me prirent feules un temps confrdérable, \& je reconnus que je ne devois compter parfaitement que fur les obfervations poftérieures. 


\section{MESURE DES TROIS PREMIERS}

Ce ne fut donc que le 29 Novembre que je commençai à obferver de fuite. Le 3 Décembre, je pûs tirer un premier réfultat de cing obfervations, dont trois avoient été faites, le limbe du Secteur étant tourné vers l'orient, \& deux, le limbe tourné vers l'occident. Je me hâtai de les communiquer à M. Bouguer, par un exprès que je lui dépêchai à Cotchefqui; mais ayant retourné l'inftument une feconde fois, le 8 , pour le remettre dans fa première fituation, le limbe vers l'orient, \& m'affurer par-là s'il n'avoit point varié dans le temps de la première inverfion, je fus extrêmement furpris de trouver la diftance apparente de l'étoile au zénith, moindre de s o fecondes, que je ne l'avois obfervée huit jours auparavant, dans la même pofition de l'inftrument. Je n'avois fait aucun changement volontaire au Secteur, \& fa folidité étoit à toute épreuve: je ne pûs donc m'empêcher de croire que quelqu'un y avoit touché à mon infú, \& de fortes raíons me confirmèrent dans ce foupçon. Mais ce qui ne me permit plus de douter du fait, c'eft qu'ayant pris depuis ce moment des mefures, pour qu'à l'avenir perfonne n'entrât dans l'obfervatoire, qu'en ma préfence, je ne remarquai plus aucune pareille variation dans la diftance de l'étoile au zénith, pendant plus de quatre mois que je continuai à obferver, toumant \& retournant alternativement le Secteur en fens contraire.

Ces cinq premières obfervations ne pouvant $f e$ lier avec celles qui les fuivirent, elles forment un rćfultat à part; mais comme celui - ci n'avoit pas été confirmé, ainfi que tous les autres, pas deux inverfions de l'inftrument; que d'ailleurs l'obfervation du 3 Déc. eft notée défectuéufe fur mon journal; que je n'avois pas encore réuffi à me garantir fùrement des variations de la 
parallaxe des fils; que ma Méridienne n'étoit pas encore bien vérifiée, \& qu'enfin au fecond détour de l'influment, javois trouvé dans la hauteur de l'étoile une différence de i o fecondes, dont la caure ne m'étoit pas évidemment connue; je regardai dès-lors ce premier réfultat comme fufpect, \& j’en juge encore de même. Au refte, puifque les cinq obfervations dont il eft tiré s'accordent paffablement entr'elles, \& qu'en les réduifant à la même époque que les fuivantes, ce premier réfultat ne diffère pas de l'autre d'une feconde, comme on le voit par la Table, il devient indifférent d'y avoir égard ou non; \& dans l'un \& l'autre cas, on tirera toujjours. les mêmes conféquences.

Mon dernier réfultat eft tiré de vingt-deux obfervations, faites depuis le 8 Déc. I 742 , jufqu'au I 7 Mars I 743 . De ces vingt-deux obfervations, douze ont été faites, le limbe du Secteur étant tourné à l'orient, à différentes reprifes : elles font entre-mêlées de dix autres, faites tandis que le limbe étoit tourné vers l'occident. M. Bouguer, qui partit de Quito pour revenir èn Europe à la fin de Févr. 1743, ne reçût à Quito que la communication de mes obfervations de Déc. I 742 , \& Janv. I 743 . Je continuai d'obferver à Tarqui en Février \& Mars, avant que d'avoir appris fon départ. Le dernier exprès que je lui dépêchai de Tarqui, \& qui lui portoit la fuite de mes obfervations, ne le trouva plus à Quito; mais jai fú depuis que mes lettres l'avoient atteint fur ta route de Carthagène, ou dans cette ville, avant fon embarquement pour l'Ife de Saint-Domingue.

On peut remarquer, que depuis le 17 Déc. I 742 , que je retournai le Secteur pour la première fois, jufquan 17 Mars Cuivant, l'inftument changea quatre fois de fituation; que dans' .

Gg ij 
cet intervalle de temps, qui comprend dix-neuf obfervations, la plus grande différence entre les diftances au zénith, obfervées \& réduites au premier Janvier I 743 , excède à peine deux fecondes; \& que les quatre dernières obfervations, faites fans être obligé d'éclairer les fils, s'accordent dans la feconde, avec les précédentes, faites à la lumière d'une bougie, laquelle, comme je l'ai déjà dit (art. $X I V$, page I 74 ), peut caufer des réfractions irrégulières, fil l'on n’ufe pas de grandes précautions.

Quili me foit permis de remarquer encore, qu'alors nous n'étions pas initruits de la manière de calculer-l'effet de l'aberration de la lumière; ce qui nous met entièrement à l'abri du foupçon de nous être fait illufion à nous-mêmes, en eftimant fur le limbe ou fur le cadran du Micromètre, les quantités apparentes, de la manière la plus propre à favorifer l'accord de nos obfervations. Avant qu'elles fuffent réduites à une même époque, il y avoit entre quelques-unes des miennes, comme entre celles des I 7 Déc. \& 2 I Févr. (Voy.Tab. préc.) des différences apparentes, de plus de 9 fecondes; au lieu qu'elles fe font prefque entièrement évanouies, depuis que mes obfervations, telles que je les avois communiquées dans le temps à $\mathbf{M}$. Bouguer, ont été corrigées pour l'aberration de la lumière, $\&$ pour la nutation de l'axe terreftre, par des théories qui nous étoient alors inconnues.

Si je faifois un choix entre mes obfervations de Tarqui, je tirerois la diftance de l'étoile au zénith, des dix-neuf dernières obfervations de la Table précédente; \& cela par les raifons que j’ai déjà infinuées: je trouverois alors cette diftance de $\mathrm{I}^{\mathrm{d}} \mathrm{L}_{\mathrm{I}} \mathrm{I}^{\mathrm{l}}$. I $\mathrm{O}^{\prime \prime}, 7$ vers le Nord; mais comme le fecond réfultat de la Table, tiré des vingt-deux dernières obfervations, eft $\mathrm{I}^{\mathrm{d}} 4 \mathrm{I}^{\prime} \mathrm{x}^{\prime \prime} 2 \frac{\mathrm{x}}{2}$, \& par conléquent ne diffère pas de cette. conclufion, d'une 
demi-feconde, il importe peu de faire ce choix ou de ne le pas faire. Soit donc qu'on corrige ou non le demier réfultat, on trouvera quili s'éloigne à peine d'une feconde de celui de

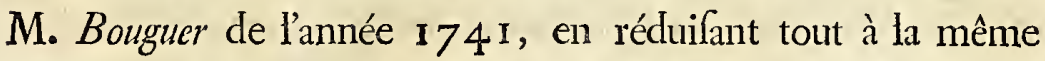
date (art. $X V$, page 182 ). Ainfi il paroît qu'il n'y a rien à defirer fur la précifion des obfervations faites à Tarqui par $M$. Bouguer en $174 \mathrm{I}$, \& par moi en 1742 \& I 743 .

Il ne me refte plus quà comparer ces dernières de $174^{2}$ \& 1743 à leurs correfpondantes \& fimultanées, faites à $C o t$ chefqui par M. Bouguer, \& rapportées article XVI; page r 83 \&. fuiv, pour tirer des unes \& des autres l'amplitude de l'arc du Méridien, intercepté entre les parallèles des deux obfervatoires.

\section{A R T I C L E XXII.}

Détermination de l'amplitude de l'arc du Méridien, compris entre les Paralleles de Cotchefqui

$$
\text { \& de Tarqui, }
$$

$P$ ar toutes les obfervations correfpondantes, faites en ces deux licux en 1742,1743 , \& réduites au premier Janvier $1>43$.

IA diftance apparente de l'étoile $\varepsilon$ d'Orion au zénith de Cotchefqui, réduite au premier Janvier 1743, a été conclue par les obfervations de M. Bouguer (art. XVI, page I 86), de

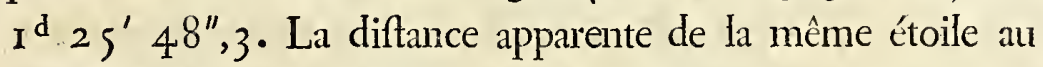
zénith de Tarqui, réduite à la même époque, a été trouvée par mes obfervations (article précédent), de I d 4 I' $^{\prime}$ I $0 ", 7 \bullet$ L'étoile étoit entre les zéniths des deux obfervateurs: c'eft - àdire; au Nord de Tarqui, \& au Sud de Cotchefqui; il faut denc ajoûter les deux diftances; pour avoir l'amplitude de l'arc 
du Méridien, compris entre les parallèles des deux obfervatoires, \& on aura $3^{\text {d }} 6^{\prime} 59^{\prime \prime}$. Mais les diftances obfervées n'étoient qu'apparentes : elles ont dû être diminuées chacune d'environ une feconde, par la réfraction qui faifoit paroître l'étoile plus près du zénith qu'elle n'étoit en effet; ainfi il y a encore 2 fec. à ajoûter à la fomme des deux diftances apparentes, - pour en conclurre l'amplitude vraie de l'arc; \& elle fera par conféquent de $3^{\mathrm{d}} 7^{\prime} \mathrm{I}^{\prime \prime}$. Tel eft le réfultat qu'on tirera en prenant un milieu entre toutes les obfervations faites de part \& d'autre; pendant plus de troís mois.

\section{A R T I C L E X X III.}

Autre détermination de l'amplitude de l'arc du Méridien, compris entre les Paralleles de Cotchefqui

$$
\text { \& de Tarqui, }
$$

Par les feules obfervations fimultanées, fans aucune réduction.

Pour réduire les obfervations faites à Cotchefqui \& à Tarqui pendant le cours de plufieurs mois, à la même époque, il a fallu avoir égard aux équations de la préceffron des E'quinoxes, de l'aberration de la lumière, \& de la nutation de l'axe de la terre. On ne peut guère aujourd'hui former de doutes raifonnables fur des théorịes reçûes de tous les Aftronomes, \& confirmées, d'un aveı unanime, par les plus modernes \& les plus fubtiles obfervations ( $a r t . V, p .127$ ); cependant $\mathrm{fr}$ on craignoit que la' multiplicité des élémens qui entrent dans ces. calculs, ou quelquautre variation, foit optique, foit réelle, dont les loix nous feroient inconnues, pût jeter quelqu'incerti- 
tude fur la conclufion précédente; les oblervations fimultanées proprement dites, celles qui ont été faites précíément les mêmes nuits, aux deux extrémités de l'arc du Méridien, nous fourniffent un moyen direct de conclure l'amplitude de cet arc, fans aucune réduction, \& indépendamment de toute hypothère. C'eft-là fur-tout ce que j'avois en vâe, lorfque je fis tant d'inftances à M. Bouguer (Introduction hiflorique, année 1742) pour l'engager à répéter au Nord de la Méridienne nos anciennes obfervations, dans le même temps que j’irois les répéter. au Sud; ce qui a été heureufement exécuté : mais il y a une attention à faire, pour employer avec fuccès cette nouvelle méthode de conclure l'amplitude de l'arc, fans aucune réduction.

En prenant, comme on a fait dans l'article précédent, un milieu entre un grand nombre d'obfervations, on court peu de rifque de fe tromper; \& quand même il y en auroit dans ce grand nombre quelques-unes de fenfiblement défectueufes, le moyen réfultat feroit à peine altéré : puifque l'excès, ou le défaut de celles-ci fe partageant entr'elles \& toutes les autres, changeroit peu le réfultat. Il en feroit de même fi on en avoit un grand nombre de fimultanées: on tireroit de chaque couple d'obfervations, faites les mêmes nuits dans les deux obfervatoires, autant de différentes amplitudes de l'arc cherché; \& alors l'amplitude moyenne entre toutes, différeioit néceffairement fort peu de la.véritable; mais comme nous n'avons qu'un petit nombre d'obfervations, faites les mênes nuits aux. deux extrémités de l'arc, il eft très-important de choifir celles qui ont le plus grand caractère d'exactitude.

On peut voir, en comparant la Table des dernières obfervations de M. Bouguer à Cotche (qui (art. XVI, pp. I 83 \& I 84), 
224 MESURE DES TROIS PREMIERS à celle de mes obfervations correfpondantes à Tarqui (art. $X X 1$, pp. 2 I $\&$ 2I 6 ); que nous n'en avons eu de fimuitanées que les nuits des 29 \& 30 Novembre, \& des 2, 8, 9. \& 17 Décembre dans les déux fituations inverfes de nos Secteurs.

Je ne ferai aucun ufage des obfervations du 29 \& du 30 Novembre, par tes raifons que j’ai expofćes dans l'article précédent, qui me les ont fait abandoṇner. Et une preuve évidente quili y a eu erreur, ces deux nuits-là, ou l'une des deux nuits, au moins dans l'une des deux obfervations fimultanées; c'eft ce que M. Bouguer \& moi, nous trouvâmes tous deux la nuit du 3 o Nov. la diftance de l'étoile au zénith, chacun d'environ deux fecondes plus grande que la veille; ce qui eft impoffible, puifque l'étoile qui étoit entre nos deux zéniths ne pouvoit s'éloigner de l'un des deux, fans s'approcher de l'autre.

J'ai pareillement lieu de me défier de l'exactitude des obfervations du 2 Déc. toute autre raifon à part, en ce qu'elles different de $4^{\prime \prime}$ de celles qui les ont précédées ou fuivies immédiatement le 3 \& le 5 . Je n'en tirerai donc aucune conféquence.

Il refte, parmi les obfervations faites les mêmes nuits à Tarqui \& à Cotchefqui, celles du 8, du $9 \&$ du I 7 Décembre; qui n'ont pas les mêmes fujets de reproche. Nous trouvâmes le 9, M. Bougụier \& moi, chacun de notre côté, les mêmes diftances au zćnith que la veille: nos obfervations du I 7 , 'faites dans une fituation contraire des deux inftrumens, s'accordent aufl avec celles qui les ont fuivies immédiatement. Ce font celles que je choifis : \& voici comme j'en tire l'amplitude de l'arc compris entre les parallèles de nos deux obfervatoires, indépendamment de toute ćquation; \& même, fans employer la diftance vraie de l'étoile au zénith de chaque lieu.

Observations 


\section{O BSERVATIONS SIMULTANEES}

\section{Aux deux extrémités de la Méridienne.}

\section{Amplitude de l'Arc célefte, compris entré les deux Zéniths.}

Les 8 \& 9 Décembre $1743: M$. Bouguer obferva la diftance de l'étoile $\varepsilon$ d'Orion au Zénith de Cotchefqui du côté du Sud; égale à la valeur du demi - arc tracé fur fon Secteur, de $1^{\mathrm{d}} \cdot 25^{\prime} 55^{\prime \prime}$, plus par le Micromètre 59". Donc de $1^{\mathrm{d}} 26^{\prime} 54^{\prime \prime}, 0$

Les mêmes nuits: j’obfervai la diftance de la mêne étoile au zénith de Tarqui, du côté du Nord; égale à la valeur du demi-arc de mon Secteur, $\left.1^{d} 4^{\prime}, 7^{\prime \prime}, 5\right\}$ plus 88 parties du Micromètre $=$ $+38,6\}$ $\left.\begin{array}{c}1^{\mathrm{d}} 26^{\prime} 54^{\prime \prime}, 0 \\ 4^{1} \\ 4^{6},\end{array}\right\}$

Sonme des deux diftances obfervées; égale à l'Amplitude apparente de l'arc du Méridien, compris entre les Parallèles des deux obfervatoires, \pm l'erreur de la pofition des lunettes des detux Secteurs. ........

Le 17 Décembre: les deux inftrumens étant retournés, $\&$ dans une fituation contraire à la précédente; $M$. Botrguer oḅferva, à Cotchefqui, la diftance de la même étoile au zénith, vers le Sud, égale au demi-arc de fon Secteur, de $I^{d} 2 \varsigma^{\prime} 55^{\prime \prime}$, moins par le Microniètre I' 1 6": en tout...

A Tarqui, la même nuit : j’obfervai la diftance de la mêne étoile au zénith vers le Nord, $\left.1^{d} 4^{I^{\prime}} 7^{\prime \prime}, 5\right\}$ moins $65 \frac{T}{2}$ parties du Micromètre $\left.=-28,7\right\}$

Somme des deux diftances obfervées, égale à l'Amplitude apparente de l'arc du Méridien, compris entre les deux zéniths, $\mp *$ f'erreur đ̄es Iunettes des deux Secteurs... $3^{\mathrm{d}} 5^{\prime} 17^{\prime \prime}, 8$

Double Amplitude de l'arc, l'erreur des deux Secteurs corrigée . . . 6 6 $13^{\prime} 57^{\prime \prime}, 9$

Vraie Anplitude de l'arc, fauf la réfraction......... $3658,9 \frac{2}{2}$

Sonme des deux réfractions qui ont diminué l'apparence de chácune des deux diftances au zénith . . . . . . . . . . . . 2

Amplitude vraie de l'arc, réfraction corrigée .........

* L'Inftrument étant retourné, l'erreur de la pofition de la lunette doit être en fens casso araire à la précédente, \& par conféquent de figne différent. 


\section{MESURE DES TROIS PREMIERS}

Donc, négligeant la fraction, l'amplitude vraie de l'arc; terminé par les cercles parallèles, qui paffent par les obfervatoires de Tarqui \& de Cotchefqui, eft de $3^{\mathrm{d}} 7^{\prime} \mathrm{I}^{\prime \prime}$, en là tirant uniquement des obfervations fumultanées; la même chofe précifément quion"a déjà trouvée par le réfultat moyen de toutes les obfervations correfpondantes, fondues enfemble, \& réduites à la même époque, en leur appliquant les équations.

Dans l'extrait de nos opérations, que M. Bouguer a donné dans les Mémoires de 1744 , il a conclu la même amplitude; \& probablement par une combinaifon de nos obfervations, différente de la mienne. Il a trouvé cette amplitude moindre d'une feconde par fes obfervations de l'étoile a du Verfeau, \& plus grande de deux fecondes par l'étoile $\theta$ d'Antinoiis. Comme il ne m'a point communiqué les obfervations qu'il a faites de ces deux étorles à Cotche fqui, je n’ai pû les comparer à celles que je fis à Tarqui dans le même temps : \& qui d'ailleurs font en petit nombre, parce qu'elles paffoient alors en plein jour; \& que n'étant que dela $2^{\mathrm{e}} \& 3^{\mathrm{c}}$ grandeur, je ne les apercevois que très-rarement. La petite quantité, dont l'arc conclu par ces deux étoiles, diffère en plus \& en moins de l'arc conclu par \& d'Orion, ne fert qu'à confirmer la première dćternination, d'autant plus que c'eft fur-tout aux obfervations d'є d'Orion que nous nous fommes attachés; \& que les correfpondantes \& fimultanées, faites en même temps aux deux extrémités de l'arc, font, de l'aveu de M. Bougucr, celles auxquelles toutes les circonftances nous obligent de donner la préférence. 


\section{A R T I C L E XXIV.}

Détermination de la longueur du degré du Méridien aux environs de l'Équateur.

Nous venons de trouver l'amplitude de l'arc compris entre les deux cercles parallèles qui paffent par les obfervatoires de Cotchefqui \& de Tarqui, de $3^{\mathrm{d}} 7^{\prime} \mathbf{1}^{\prime \prime}$, \& la diftance de ces deux mêmes parallèles a été trouvée (Part. I, art. $X X V I I, p .104$ ) de 176950 toifes. II n'y a plus qu'à comparer ce nombre de toifes à celui des degrés, minutes \& fecondes de l'arc correfpondant, pour en concluare la valeur du degré.

J'avois remarqué, dès le temps de mes premiers calculs à Quito, que par les différens choix, \& les diverfes.combinaifons des obfervations, on trouvoit l'amplitude de l'arc plus grande ou plus petite d'environ une feconde que $3^{d}{ }^{d} 7^{\prime} \mathrm{o}^{\prime \prime}$; ce qui m'engagea à calculer la valeur du degré fur ce nombre rond de minutes; qui tenoit le milieu entre les différentes déterminations*. En divifant 17695 o toifes, longueur de l'arc, par fon amplitude; fuppofée de $3^{\mathrm{d}} 7^{\prime} \mathrm{o}$ ", ou de 187 minutes, on trouvera à proportion, Ia longueur du degré de $56775^{t}, 42$. Si on ajoûte une feconde de plus au divifeur; c'eft-à-dire, fi on divife la même longueur de la mefure géométrique par $3^{\mathrm{d}} 7^{\prime} \mathrm{r}^{\prime \prime}$, amplitude de l'arc telle qu'elle a été conclue, tant de nos dernières obfervations correfpondantes, prifes toutes enfemble, que des.feules

* C'eft fur ce pied-là que j’ai fait le calcul dans l'extrait de mes obfervations, daté du Port de Jaën le 3 Juillet 1743 , envoyé en Europe pour être remis à l'Académie, fi je mourois en chemin, \& dont une copie eft reftée en dépôt à Quito. Voy. Introd. hiffor, Juillet ' 1743 . 
228 MESURE DES TROIS PREMIERS

obfervations fimultanées, on trouvera la longueur du degré de $5^{6} 677^{\circ} 20$; au lieu de $5677.5^{\mathrm{t}} 42$, qu'on avoit trouvées par le précédent calcul : ce qui fait voir qu'une feconde de plus dans l'amplitude de l'arc, ne diminue la longueur du degré que d'un peu plus de 5 toifes; \& qu'ainfı quelques fecondes de plus ou de moins ne la changeroient quà proportion.

T'elle eft la longueur du degré tiré de nos obfervations aftronomiques, communes à M. Bouguer \& à moi, \& de ma mefure particulière des Triangles; mais cette longueur eft celle du degré au niveau de Carabouron, le plus bas de nos Signaux, \& le terme feptentrional de notre première Bafe; \& ce Signal étoit élevé de $\mathbf{2} 26$ toifes (Part. l, art. XIV, page 52) au deffus de la furface de In mer.

If refte à réduire notre mefure à ce niveau, pour la pouvoir comparer à celle des degrés mefurés en France \& en Lapponie.

Il eft évident que le degré au niveau de Carabourou, eft plus grand que le degré au niveau de la mer, dans la même raifon que le rayon de la Terre, pris depuis fon centre jufqu'à Ia hauteur de Carabourou, eft plus grand que le rayon de la Terre, terminé par Ia furface de la Mer. Suppofons, comme je l'ai déjà fait en pareil cas (Voy. la Note, Part. I, art. 1, p.8). que le rayon de la Terre, près de l'Équateur, foit de 3268319. toilęs, au niveau de la mer; (les hypothèles les plus différentes fur la figure de la Terre n'apporteront point de différence fenfrble dans la conféquence que nous allons tirer de notre fuppofition, pour la réduction du degré). Ajoûtons r 226 toifes au rayon fuppofé, nous aurons le rayon au niveau de Carabourou, de 3269545 toifes, dont 1226 toifes eft à peu près Ia $2666 \frac{1}{2}$ partie. Il y a donc $\frac{\mathrm{r}}{2666 \frac{1}{2}}$ ì retrancher de la longueur 
du degré mefuré, \& rapporté au niveau de Carabourou; c'eftà-dịre, à peu près $21 \frac{1}{4}$ toifes à ôter de $56770^{t} 20$. Ainfi if reftera 56749 toifes, ou, en nombre rond, 56750 toifes, pour la longueur au niveau de la mer, d'un des premiers degrés du Méridien : je dis d'un des premiers degrés, parce que dans les hypothèfes les plus diverfes de la courbure de la Terre, Jes trois premiers degrés diffèrent à peine d'une toife.

\section{A R T I C L E XXV.}

De l'erreur poffible dans la détermination précédente de la valeur du degré du Méridien.

SI quelque erreur a pû fe gliffer dans la détermination précédente de la valeur du degré, elle provient néceffairement, ou du défaut de la mefure aftronomique de l'amplitude de l'arc du Méridien, ou du défaut de la mefure géodéfique de la tongueur du même arc.

Si on fe rappelle tout ce qui a été dit (Part. II, art. $V$, page.126, \& art. XVII, XVIII, XIX, XX, XXI) fur les obfervations par lefquelles l'amplitude a été conclue; leur nombre, leur choix, les précautions qui ont été prifes, l'accord de deux différens Obfervateurs, en différens temps, avec divers inftrumens, \& en variant les procédés; je crois qu’on m'accordera fans peine, qu'on peut raifonnablement fuppofer, quil n'y a pas plus de trois fecondes d'erreur à craindre fur l'amplitude obfervée de l'arc de 3 degrés 7 minutes. Cette erreur n'eft que poffible; fuppofons-la réelle, \& doublons-la encore, elle fera de fix fecondes fur un arc de $3^{\mathrm{d}} 7^{\prime}$; c'eft-à-dire,

Hh iij 
Examinons maintenani quelle erreur peut comporter ma mefure géodéfique. On a vû (Part. I, art. $X X V \& X X V I$, page 93 \& (uiv.) que $1 \mathrm{i}$ on en juge par la différence d'une toife, trouvée entre la longueur de la feconde Bafe conclue par le calcul, \& fa longueur actuellensent mefurée; toute l'erreur qu'on auroit à craindre, \& même avec très-peu de vrai-femblance, ne feroit que de i 8 töfes fur $17695^{\circ}$; ce qui ne revient pas à 6 toifes par degré. Je n’infifte pas fur ce que jai fait voir d'ailleurs (Part. I, art. XXIII, page 86); que j’aurois pû réduire cette différence à la moitić.

Servons-noús d'un nouveau moyen pour évaluer cette même erreur, en comparant ma mefure trigonométrique à celle des deux autres Académiciens.

Nous fommes d'accord, M. Bouguer \& moi, dans la feconde (art. $X X I 11$, page 226). fur l'amplitude de l'arc du Méridien, tirée de nos obfervations communes par diverfes combinaifons. Nous ne pouvons donc différer que fur la longueur du même arc, conclue par nos diverfes mefures d'angles. Mámoires ae M. Bouguer fait la longueur du degré au niveau de la mer l'Académie de 1744 , page de 56746 toifes; je lai trouvée, par ma mefure particulière, de 56749 toiles (art. précéd.): c'eft-à-dire, plus grande que lui Mémoires de de trois toifes. M. Bouguer ajoûte 7 toifes par degré pour l'équal'Acad. 1744 ' tion de la variation de la Toife, à laquelle j'ai cru ne devoir pas
page 297. -avoir égard, par les raifons que jai expolées (Part. I. art. XXII, page 80 \& fuiv.). Par cette dernière détermination, fon degré, au lieu d'être de 3 toiles plus petit que le mien, eft donc de 4 toifes plus grand; \& comme je me fuis arrêté au nombre rond de 56750 toifes, il s'enfuit, que toute correction faite, la 
melure trigonométrique de $M$. Bouguer ne differe de la mienne que de 3 toifes fur le degré, \& en excès.

Quant à la mefure géodéfique de M. Godin, non feulement elle n'a pas été exécutée avec les mêmes inftrumens que les deux autres, mais d'ailleurs fa Suite des Triangles eft différente de celle que $M$. Bouguer \& moi, nous avons, chacun, mefurée à part : outre que celle de $\mathrm{M}$. Godin contient quelques Triangles de plus vers le -Nord, \& quelques autres de moins vers le Sud (Voyez Partie 1 , article III, page .1 2). Cependant comme nos trois mefures ont un grand nombre de points communs, on peut comparer une grande portion de celle de M. Godin, à celle de M. Bouguer \& à la mienne. Pour éviter la longueur du calcul \& les réductions, je me contenterai de comparer la diftance des parallèles des Signaux du Coraçon \& de Boteran, fitués l'un à od $32^{\prime}$, \& l'autre à $2^{\mathrm{d}} 35^{\prime}$ de latitude auftrale : je la trouve toute calculée dans Pes Triangles, le livre d'obfervations déja cité, imprimé à Madrid en I 748 , page $2 I 3$. Si l'on ajoûte les diftances des parallèles des Signaux intermédiaires, fuivant le calcul qu'en a fait $M$. le Commandeur Don Georges Juan, qui a toûjours opéré fur le terrein conjointement avec M. Godin; on aura la diftance totale entre le Parallèle du Coraçon \& celui de Boueran, réduite au niveau de la mer, de 1 I 753 I $^{\mathrm{t}}, 08$. Or cette même diftance, au niveau de Carabourou, eft, par le calcul de mes Triangles (Part. I, art. $X I X$, page 68), de 135 I $93^{\mathrm{t}}, \mathrm{r} 3$,

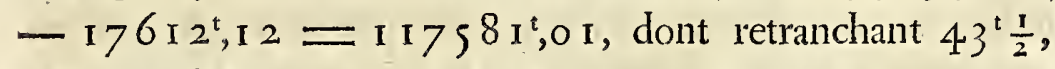
pour la réduire au niveau de la mer, il reftera i I $7537^{t}, 51$; c'eft-à-dire, environ 6 toifes de plus que par les Triangles de M. Godin, fur une étendue qui comprend plus de deux degrés. 


\section{MESURE DES TROIS PREMIERS}

Ma mefure trigonométrique n'excède donc celle de $M$. Gódin que de 3 toifes fur la longueur d'un degré. Nous venons de voir que la mienne eft plus courte que celle de $M$. Bouguer de la même quantité; elle tient donc précifément le milieu entre les mefures des deux autres Académiciens.

Cette différence entre trois mefures exécutées féparément \& avec divers inftrumens, n'eft pas la dix-neuf millième partie de la quantité mefurée. Faut-il d'autre preuve de la grande exactitude des trois opérations?

Puifque ma mefure géodéfique eft moyenne entre les deux autres, je ferois en dioit de la regarder comme exacte; mais fuppofons que Ia différence de trois toifes, en plus ou en moins, provienne d'une erreur qui foit toute entière de mon côté; cette erreur pourroit diminuer celle de 32 toifes par degré, que nous avons fuppofée dans la mefure aftronomique : car il eft auffr probable que cette feconde erreur fe trouve en fens contraire, que du même fens que la première; mais ne prenons point encore ici de milieu, \& fuppofons au contraire que les deux erreurs s'ajoùtent, au lieu de fe compenfer en partie.

Que réfultera - $t$ - il de toutes ces fuppofitions forcées, d'erreurs plus grandes que celles qu'on peut craindre avec quelque fondement? C'eft qu'il ne feroit pas phyfiquement impoffible que l'erreur, dans ma détermination de la valeur du degré du Méridien, montât d'une part à 32 toifes, \& de l'autre à 3 toifes, on en tout à 35 toifes; mais quil eft, fans comparaifon, plus vrai-femblable qu'elle eft beaucoup moindre; \& très-poffible qu'elle foit $f_{1}$ petite, qu'elle ne mérite aucune confidération. 
Jé pourrois encore déterminer la valeur du degré par mes feules obfervations particulières: en comparant la longueur d'un arc du Méridien, de I 62 I 28 toifes (a), tirée de la mefure de mes Triangles ; à l'amplitude du mêne arc, conclue de $2^{\mathrm{d}} \mathrm{SI}^{\prime} 2 \mathrm{~S}^{\prime \prime}(\mathrm{b})$, par les deux diftances de la même étoile au zénith, que j’ai obfervées à Quito en Juillet I 742, \& à Tarqui en Janv. I 743: \& de deux fecondes plus grande en corrigeant la réfraction. En ce cas je trouverois la longueur du degré, réduite au niveau de la mer, de 567 i 7 toifes, au lieu de 56749, ou 56750 ; c'eft-à -dire, moindre de 32 ou 33 toifes que celle qui réfulte de ma précédente détermination; mais comme je ne mets pas la dernière, en parallèle avec celle que jaa déduite de nos obfervations fimultanées aux deux extrémités de la Méridienne, je ne fais mention de celle qui mappartient en propre, que pour faire voir qu'elle s'accorde avec celle à laquelle je me fuis arrêté, avec une différence moindre

(a) Diftance du Signal de Clinan à la Perpendiculaire à la Tour de Ia Mercy de Quito (Part. I, art. XIX, pages 68 \& 69), 162995 toifes, dont il faut ôter, pour la réduire à la diftance des deux obfervatoires de Quito \& de Tarqui, premièrement 2 toifes (Part. II, art. XIV, p. 175), parce que le point $L$ du plan de Quito, où a été faite l'obfervation aftrononique, eft deux toifes plus auftral que la Tour; puis 857 toifes dont Cliinan eft plus aufral que l'obfervatoire de Tarqui (Part. I, art. XXVII, page 103), \& enfin dont il faut encore retrancher 8 toifes pour la convergence des Méridiens (Ibid. page 104.).

(b) Diftance apparente d' $\varepsilon$ d'Orion au zénith du point $L$, où j'ai obfervé feul à Quito, deux toifes plus au Sud que le centre $X$ de la Tour de la Mercy: I I I ${ }^{\prime}$ i $5^{\prime \prime}$ vers le Sud en la réduifant au I ${ }^{\circ}$ Janv. 743 (Part. Voyez le plat de Quits. $I I$, art. $X I V$, pages $\left.1>I \& \sigma^{\prime}>6\right)$. Diftance apparente de la même étoile au zénith de l'obfervatoire de Tarqui, $\mathrm{I}^{\mathrm{d}} 4 \mathrm{~L}^{\prime} \mathrm{I}^{\prime} \mathrm{I}^{\prime \prime}$ vers le Nord pour le niême temps (Part. II, art. XXI, page 2 I 6). Donc Somme des deur diftances au zénith, ou anplitude apparente de l'arc, $2^{\mathrm{d}} \varsigma^{\prime \prime} 2 \varsigma^{\prime \prime}$. 


\section{MESURE DES TROIS PREMIERS}

que celle que j’ai affignée aux limites des erreurs pofibles.

J'omets, par la même raifon, plufieurs obfervations du Soleil \& des mêmes étoiles, faites aux deux extrémités de ta Méridienne avec un Quart-de-cercle de trois pieds de rayon, defquelles je pourrois tirer une valeur du degré trèspeu différente de celle que je regarde comme la véritable.

Enfin je puis encore comparer la valeur de mon degré à celle que lui attribuent $M$ rs les deux Officiers Efpagnols, nos Compagnons de voyage. Leur détermination eft tirée de deux différentes mefures trigonométriques (Voy. Part. I, art. 1II, p. 13 ), dont ils comparent le réfultat moyen à l'amplitude d'un arc de $3 \frac{1}{2}$ degré, conclue de l'obfervation aftronomique qui Jeur eft commune avec M. Godin, à l'extrémité auftrale de leur ar'c, \& de celle qu'ils ont faite feuls à l'extrémité boréale de la Méridienne. Ils fixent la valeur du degré du Méridien au niveau de la mer à 56768 toifes (Objerv. aftronom, y phyfico Madrid, 1748 , page 295); ce qui ne diffère encore que de 2 I toifes en plus, de celle à laquelle je m'en fuis tenu : en forte qu'elle tient à peu près le milieu entre la mefüre de ces Meffieurs \& la précédente, tirée de mes feules obfervations.

Je crois avoir prouvé que la valeur de $5675^{\circ}$ toifes, que j’afligne au degré du Méridien proche de l'Équateur, eft trèsapprochante de la véritable. Cependant je me contenterai que I'on m'accorde; \& je ne penfe pas que ce foit me faire grace, qu'elle n'en differe pas de plus de 35 à 40 toifes. Dès-lors la queftion de la non fphéricité de la Terre, principal motif de notre voyage, eft décidée fans aucun doute, \& elle le feroit encore, comme on va le prouver, quand on donneroit à l'erreur poffuble, des limites beaucoup plus étendues. 


\section{A R T I C L E X X V I.}

De linégalité des degrés du Méridien, \& de ce qui ere réfulte, quant à la figure de la Terre.

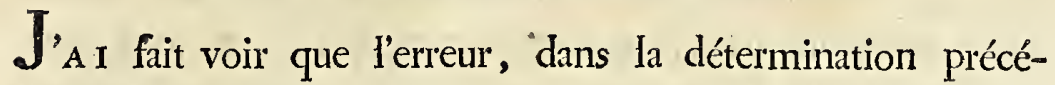
dente de la valeur du degré, ne pouvoit monter à 40 toifes, en faifant les fuppofitions les plus violentes \& tes moins vraifemblables; mais cette erreur fuit -elle beaucoup plus confrdérable, il feroit encore évident que la Terre n’eft pas fphérique, \& que l'axe qui la traverfe d'un Pole à l'autre eft plus court que le diamètre de fon E'quateur; ce qu'on exprime ordinairement, en difant que la Terre eft un fphérö̈de aplati vers les Poles, parce que cette propofition eft une conféquence néceffaire de la précédente.

Qu'on prenne la longueur du premier degré du Méridien, telle que je viens de l'établir; qu'on y ajoûte, ou qu'on en retranche $f \mathrm{l}$ l'on veut, 40 toifes, \& qu'on ta compare enfuite à quelle que ce foit des mefures des degrés du Méridien, exécutées en France. Sans entrer, quant à préfent, dans autcune difcuffion fur celle qui mérite la préférence, on trouvera toûjours que notre premier degré de latitude eft plus petit quïI n'eft en France, fous le parallèle de Paris, denviron 300 toifes.

D'un autre côté, fr on compare à celui-ci, le 66 e degré, mefuré par les Académiciens qui ont fait le voyage du Nord, on verra que ce dernier eft plus long de 300 à 400 toifes que celui de France. Les deux degrés extrêmes, l'un voifin de l'Equateur, l'autre qui coupe le cercle polaire, different donc de' 
700 toifes. Il fuffit que les Obfervateurs aient eu des yeux, pour que des différences auffi confidérables ne puiffent être attribuées à des erreurs d'obfervation.

II n'eft donc plus permis de douter que le degré du Méridien ne foit plus petit près l'Équateur que vers le Pole, \& de-là il s'enfuit néceffairement, que la Terre eft aplatie vers les Poles; \& rehauflée fous l'Équateur. Je n'infifterai pas fur les preuves d'une conféquence avouée de tous les Mathématiciens, \& que $M$. de Maupertuis, dans fon Difcours fur la mefure du degré au Cercle polaire (page 8), \& dans plufieurs autres ouvrages, à mife à la portée de tout le monde, en l'expofant de la manière la plus claire \& Ia plus fenfible; je me contenterai de faire le raifonnement fuivant en faveur de ceux à qui il n'eft befoin que de rappeler leurs idées fur cette matière. L'éloignement des étoiles fixes à la Terre eft fr prodigieux, que quelque diftance que parcourntt un Voyageur fur Ia Terre, il verroit toûjours les mêmes étoiles répondre à fon zénith, fi la furface de la Terre étoit abfolument plane: ce n'eft donc que fa courbure qui fait changer la ligne verticale de l'Obfervateur, \& varier la plus grande hauteur apparente d'une même étoile. Parcourir un degré du Méridien, c'eft faire affez de chemin vers le Nord ou vers le Sud, pour que l'étoile, qui répondoit à notre zénith, paroiffe s'abaiffer d'un degré. Ainfi plus la furface de la Terre fera plate, plus il y aura de chemin à faire fur le Méridien, pour apercevoir ce changement dans le zénith. Or les degrés du Méridien ont été trouvés, par toutes les mefures, plus longs vers le Pole que vers l'Équateur : il faut donc faire plus de chemin en approchant du Pole, que près de l'Équateur, pour parcourir 
un degré. La Terre eft donc moins courbe, \& approche donc plus d'un plan vers le Pole: donc Ja Terre eft un fphéroïde aplati.

On tire la même conféquence de la comparaifon de toutes les mefures du degré du Méridien, tant de fois répétées en France, en Lapponie \& au Pérou; ce qui décide, fans appel, Ia queftion qui partageoit les Savans depuis près d'un fiècle. Mais quelle eft la mefure de cet aplatiffement, \& dans quel rapport croiffent les degrés de latitude en approchant des Poles? C'eft ce que nous ignorons encore, \& ce qu'il n'eft peut-être pas poffible de favoir; au moins fans avoir un beaucoup plus grand nombre de degrés mefurés.

Toutes les théories de la figure de la Terre s'accordant à faire le Méridien elliptique, on a été fondé à croire que pour en déterminer la courbure, la mefure de deux degrés fuffifoit; \& quiil falloit feulement, pour rendre la détermination plus exacte, que les deux arcs mefurés fuffent à la plus grande diftance poffible entre l'Écquateur \& le Pole. Voilà quel a été le motif des deux grands voyages, entrepris pour la mefure de la Terre. On étoit d'accord fur la longueur moyenne du degré en France; je dis fur la longueur moyenne, car.1a différence des degrés voifms eft trop petite pour être reconnue immédiatement \& furement par les obfervations. If fembloit donc qu’il n'y eût plus qu’à comparer le degré moyen du Méridien en France, aux degrés qui devoient en différer le plus, foit par défaut, foit par excès. On a été chercher ces degrés fous l'E'quateur d'une part; \& de l'autre, le plus près du Polẹ qu'il a été poffible: \& quand même la différence de I'un , ou de l'un \& l'autre de ces deux degrés à celui de France auroit pû échapper aux obfervations, on jugeoit, avec raifon, 
qu'au moins la différence entre les deux degrés extrêmes, nè : pourroit manquer de fe manifefter, pour peu qu'elle funt notable. Elle s'eft manifeftée en effet, \& d'une manière trèsfenfible; non feulement entre les degrés extrèmes, mais encore de plus de 300 toifes, entre chaque degré, \& le degré moyen : comme il a déjà été remarqué. Chaque comparaifon qu'on peut faire, entre deux arcs mefurés à une grande diftance, fournit une nouvelle preuve de l'inégalité des degrés croiffants de l'E'quateur au Pole; \& par conféquent de l'excès du diamètre de l'Équateur fur l'axe du fphéroïde. Cependant il s'en faut beaucoup que toutes ces comparaifons donnent. par le calcul un même rapport d'inégalité entre l'axe de rotation \& le diamètre de l'E'quateur. Si nous n'avions aujourd'hui qu'une des deux mefures du degré du Méridien, ou au Pérou, ou en Lapponie, à comparer à cefle du degré moyen de France, on ne fe feroit peut-être pas avifé de douter que l'ellipfe, qui réfultoit de cette comparaifon, pût ne pas donner Ja vraic courbure du Méridien; mais les trois mefures des degrés de latitude en Lapponie, en France \& au Pérou, nous ont appris qu'on trouvoit autant de différentes elliples qu'on peut faire de différentes combinaifons des degrés mefurés; c'eft ce que nous allons bientôt prouver, en appliquant aux mefures, exécutćes fous le Cercle polaire, en France \& fous 1 'Équateur, les formules données par $M$. de Maupertuis (Mém. de l'Acad. I737, page 463, \& Mef. du deg. du Mérid. au Cercle polaire, Liv. I, chap. IX, page 127. 


\section{A R T I C L E XXVII.}

Des différentes mefures du degré du Méridien en France. Erreur dans la me fure aftronomique de $M$. Picard.

Avan t que de comparer entr'eux les degrés du Méridien, mefurés à diverfes latitudes, il eft important de remarquer, quili y a eu plufieurs différens arcs du Méridien mefurés fous Le Parallèle de Paris, en divers temps, \& par différens Obfervateurs. De-là ont réfulté plufieurs diverfes mefures du même 'degré, qu'il eft à propos de diftinguer ici, pour prévenir toute équivoque. Cette difcuffion tient de trop près au fujet que je traite, pour pouvoir être regardée comme une digreffion.

$I^{\circ}$ M. Picard, dans fa mefure de la Terre (art. $X$, page 81), détermine la longueur du degré du Méridien entre Paris \& Amiens; c'eft-à-dire, à très-peu près, celle du $49^{\mathrm{e}}$ degré de latitude, de........... 57060 toifes.

$2^{\circ}$ Si on applique aux obfervations aftronomiques, dont M. Picard a conclu la longueur du degré, les équations dont elles ont befoin, \& qu'il a négligées, ou qui étoient inconnues de fon temps, l'amplitude de fon arc fera augmentée de I I' $\frac{I^{\prime \prime}}{2}$, \& la longueur de fon degré ne fera pius que de.............. 56925 toifes. Voy. Mef. du deg. au Cerc.pol. Liv. I, shap. VIII, p. I 26 .

$3^{\circ}$ Ce même degré, conclu par la nouvelle mefure aftronomique de $M^{\text {rs }}$ de Manpertuis, Clairaut, Camus \& le Monnier (Degr. du Mérid. entre Paris or Amiens, chap. VIII, page LIV ), \& par la mefure topographique de M. Picard, eft de 
57 I 83 toifes, en négligeant la réfraction qui dininuoit l'arc apparent d'environ une feconde.

$4^{\circ}$ Et ayant égard à la réfraction, il fera de 57 r 64 toifes.

$5^{\circ}$ Enfin fi on tire la valeur du même degré, de l'amplitude de l'arc mefuré en France en 1739 par les Académiciens du voyage du Nord, \& de la nouvelle mefure géodéfique de la longueur de cet arc par $M^{\mathrm{rs}}$ Caffini de Thuri, \& de la Caille

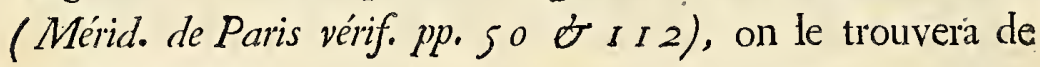
$57074 \frac{1}{2}$ toifes.

Voilà, comme on voit, quatre ou cinc déterminations du même degré, autorifées par des fuffrages d'un grand poids, \& tour à tour regardées comme les véritables.

La première de ces mefures, celle de M. Picard, de 57060 toifes, a long-temps été univerfellement reconnue pour la vraie longueur du degré d'un grand cercle de notre globe; elle a fervi à tous les calculs des Géographes, des Aftronomes \& des plus habiles Pilotes; non feulement tant que la Terre a été réputée fphérique, mais encore depuis que les théories de $\mathrm{M}^{\mathrm{r} s}$ Huygens \& Newton eurent jeté les premiers doutes fur fa fphéricité. Ce derniel même, établiffant par fes principes l'inégalité des degrés terreftres, prit la mefure de M. Picard pour celle du degré moyen du Méridien; \& avec d'autant plus de fondement, que cette mefure avoit été confirmée par M. Cafjini, qui avoit trouvé en I 7 I 8 le degré moyen entre les huit degrés de latitude, mefurés en France, de 5706 I toifes, de la même longueur, à une toife près, que M. Picard (Voy. Suite des Mém. de l'Acad. de I 7 8: De la grand. \& de la fig. de la Terre, page 247).

Il faut encore convenir qu'il n'y a pas même aujourd'hui une 
unè grande erreur à craindre en prenant ce degré pour le degré moyen dı Méridien, puifqu’il ne diffère que très-peu de la valeur qui a été affignée par les dernières mefures au degré noyen de latitude en France, lequel, dans toutes les hypothèfes, tient à peu près le milieu entre les degrés extrêmes du Méridien.

Mais en voyant le degré de $M$. Picard s'accorder, à I 4 ou 15 toifes près, avec celui que $M^{\text {rs }}$ Caffuni de Thury \& de la Caille ont fixé à $57074 \frac{1}{2}$ toifes en I 740 , par leur nouvelle mefure géographique, combinée avec l'amplitude de I'arc, déterminée l'année précédente par $\mathrm{M}^{\mathrm{r} s}$ de Maupertuis, Clairaut, Camus, le Monnier \& de Kermadec, on ne fe douteroit pas, fans doute, que cette conformité apparente n'eft dûe quà une compenfation fortuite de plufreurs erreurs trèsconfidérables, tant dans les obfervations céleftes, que dans les terreftres de M. Picard; \& on ne préfumeroit pas vrai-femblablement, que l'amplitude de l'arc, intercepté entre les Cathédrales de Paris \& d'Amiens, qui réfulte des mefures de cet Aftronome, excedât de près de 20 fecondes celle qui a été trouvée par les Académiciens du voyage du Nord; \& que la diftance de Paris à Amiens, calculée fur les Triangles de M. Picard, furpaffât de près de r oo toifes celle qui fe conclud des nouvelles mefures trigonométriques de $\mathrm{M}^{\mathrm{rs}}$ Caffini de Thury \& de la Caille.

C'eft ce qui n'a pas, ce me femble, encore été fuffifamment éclairci, \& ce qu'il eft aifé de démontrer, en rapprochant fous un même point de vîe ce qui fe trouve répandu en divers ouvrages, publiés depuis quelques années. 


\section{A R T I C L E X X V III.}

Comparaifon de la mefure de l'amplitude de l'arc du Méridien entre Paris \& Amiens, par M. Picard, à celle du même arc nouvellement mefuré en 1740.

Messieurs de Maupertuis, Clairaut, Canuls \& le Monnier, depuis leur retour de Lapponie, entreprirent, comme on fiait, de mefurer le degré du Méridien de Paris à Amiens avec le même Secteur qui leur avoit fervi à l'obfervation du degré qui coupe le Cercle polaire. Ifs trouvèrent en 1739 l'amplitude de l'arc du Méridien, intercepté entre le Parallèle de l'extrémité feptentrionale de la rue de Louis-le-Grand à Paris, \& celui du Jardin du Roy à Amiens, de I d I' r.2" (Voy. Deg. entre Paris \& Amiens, page liv). Et comme pour réduire cet arc à celui qui eft compris entre les paralièles des deux Cathédrales, il faut ajoûter I I o 5 toifes d'une part, \& $98 \frac{1}{2}$ toifes de l'autre (Ibid.): en tout I $203 \frac{1}{2}$ toifes $=\mathrm{I}^{\prime} \mathrm{I}^{\prime \prime}$; ces $\mathrm{M}^{\text {rs }}$ conclurrent l'arc du Méridien, entre les deux E'glifes, de $\mathbf{I}^{\mathbf{d}}$ $I^{\prime}$ I $2^{\prime \prime}+I^{\prime}$ I $^{\prime \prime}$; c'ét-à-dire, de I ${ }^{\mathrm{d}} 2^{\prime} 28^{\prime \prime}$ (page v)*. Dans ce calcul, on a négligé la réfraction, qui ne monte qu’à $\mathbf{I}^{\prime \prime}$; mais comme on y a eu égard dans les calculs précédens, j’en tiendrai compte dans celui - ci, pour procéder uniformément: On aura donc l'amplitude de lárc du Méridien, obfervée par les quatre Académiciens; favoir, à Paris, au nord de la rue de Louis-le-Grand, \& à Amiens au Jardin du Roy, de I d I' 13 ",

* Entre la flèche de l'E'glife de Notre-Dame d'Amiens \& la Guérite de la Tour auftrale de Notre-Dame de Paris, 
ên ayant égard à la réfraction; \& par conféquent l'amplitude de l'arc entre les deux Cathédrales de 'I ' 2' $29^{\prime \prime}$.

Voyons maintenant ce qui réfulte des obfervations de $M$. Picard. La diftance des Parallèles, ou la différence de latitude de Malvoifine \& d'Amiens, entre les deux points où M. Picard a obfervé, eft, felon cet Aftronome, de I $^{\mathrm{d}} 22^{\prime} 55^{\prime \prime}$ ( $M e$ f. de la Terre, art. $X, p .>8)$.

- Mais le pavillon de Malvoifine, qui a fervi de Signal pour Jes Triangles, eft, felon M. Picard, I 9376 toifes plus auftral que Notre-Dame de Paris (Mef. de la Terre, art. VII, pages $5>\& 58)$; \& le point où $M$. Picard obferva l'étoile à $M a l-$ voifme (art. $X$, page 77 ), étoit I 8 toifes plus auftral que le pavillon. Donc i 9394 toifes au Sud de Notre-Dame de Paris.

A Amiens, au contraire, l'Églife eft de 75 toifes plus Nord que le dieu des obfervations de M. Picard, (art. $X$, page 77 ).

II y a donc 75 toifes à ajoûter, \& I 9394 toifes à ôter; c'eft-à-dire en total, I 93 I 9 toifes à retrancher de la diftance des deux obfervatoires de M. Picard, pour réduire fon arc aux deux Cathédrales.

Or i 93 I 9 toifes, fuppofant avec M. Picard le degré de \$7060 toifes, font équivalentes à 20' I 9", donc l'amplitude apparente de l'arc, compris entre les parallèles des deux Cathédrales, doit être, fuivant la manière de calculer de M. Picard, Id $2^{\prime} 3^{\prime \prime}$; c'eft-à-dire, de $7^{\prime \prime}$ plus grande que I d 2' $29^{\prime \prime}$, qui fe déduit des obfervations des Académiciens du voyage du Nord.

Cet arc doit encore être augmenté, à caufe des mouvemens apparens de l'étoile dans l'intervalle des obfervations;

$\mathrm{Kk}$ ij 


\section{MESURE DES TROIS PREMIERS}

faites aux deux extrémités de l'arc: il y a $8 " \frac{1}{2}$ à y ajoûter pour l'aberration de la lumière, $I^{\prime \prime} \frac{1}{2}$ pour la préceffion des E'cquinoxes, \& autant pour la réfraction (Mef. du deg. auCorcle pol. shap. VIII, page I 26). Si donc M. Picard avoit fait ces corrections, il eût trouvé fon arc plus grand de I I" $\frac{1}{2}$ quili ne l'a conclu.

Donc l'amplitude de l'arc de M. Picard entre les Cathédrales de Paris \& d'Amiens, corrigée par toutes les équations, eft de ............. I ${ }^{d} 2^{\prime} 47^{\prime \prime} \frac{1}{2}$.

Celle du même arc, par les obfervations des quatre Académiciens, ayant égard à la réfraction. I 229.

Différence entre les deux amplitudes . . . $\frac{1}{1} 8 \frac{1}{2}$.

Quelque reconnues que foient l'habileté de M. Picard, \& fon exactitude dans les obfervations, elles ne peuvent balancer l'authenticité de la dernière détermination. Indépendamment des I I" $\frac{1}{2}$ d'erreur que caufe l'omiffion des équations, on fait que du temps de M. Picard, les inftrumens d'Aftronomie étoient beaucoup moins parfaits quils ne le font depuis quelques années; d'ailleurs, nous ne voyons point quili ait vérifié les divifions de celui qui lui fervit à prendre les diftances d'étoiles au zénith. Enfin, fans entrer dans le détail de la critique quon pourroit faire de fon Secteur, il eft évident que le degré de précifion quil comportoit ne peut être mis en parallèle avec la perfection du Secteur de M. Graham, qui, par la vérification faite de i 5 en I 5 minutes, dans le voyage au Cercle polaire (chap. VII, page 1 20), \& par toutes les épreuves qui en ont été faites, furpaffe tout ce qu’on a connu de plus parfait en ce genre.

Enfin le concours de quatre Obfervateurs habiles, Ia con- 


\section{DEGRES DU MERIDIEN.}

formité de leurs oblervations, répétées fur deux étoiles différentes à Amiens \& à Paris; la preuve de fait qu’ils ont donnée, par l'inverfon de leur Sećteur, à Paris, à Amiens, \& derechef à Paris (Deg. du Mérid. entre Paris \& Amiens, chap. 1II, p. xxiv \& fuiv.), que la ligne verticale n'étoit pas fujette à varier dans cet inftrument par le tranfport, comme dans les inftumens ordinaires : toutes ces circonftances ne laiffent aucum doute, que l'erreur dans l'obfervation aftronomique ne foit toute entière du côté de M. Picard.

L'amplitude corrigée de M. Picard, plus grande de i $8^{\prime \prime} \frac{\mathrm{r}}{2}$ que celle du même arc, déterminée par les quatre Académiciens, devroit produire une différence de 3 oo toifes, entre les longueurs du même degré, mefuré en France par M. Picard, \& par ces Meffieurs. Si donc ils n'ont conclu le degré entre Paris \& Amiens, que d'environ i 20 toifes plus long que $M$. Picard, en ne changeant rien à fa mefure géodéfrque ( Mef. du deg. du Mérid. entre Paris \& Amiens, ch. VIII, p. LIV ); ce n'eft que l'omifion quil a faite de I I " $\frac{1}{2}$ d'équation, qui a heureufement rapproché les deux déterminations. Cette omiflion réduit une différence réelle de i $8^{\prime \prime} \frac{1}{2}$, entre les deux amplitudes, à une différence apparente de $7^{\prime \prime}$, entre l'amplitude effectivement conclue par le calcul de M. Picard, \& celle qui fe déduit de l'obfervation des quatre Académiciens; mais ces 7 " fuffiroient encore pour faire trouver la valeur du degré plus grande de i i o toifes que M. Picard ne l'a jugée; fi de nouvelles erreurs, dans les mefures trigonométriques qui ont fervi à conclurre la diftance de Paris à Amiens, n'avoient donné lieu à une nouvelle compenfation. Cet examen fera le fujet de l'article fuivant.

$\mathrm{Kk}$ iij 


\section{A R T I C L E X X IX.}

Examen de la Bafe de $M$. Picard, \& de fa mefure géodéflque de la diftance de Paris à Amiens.

L'erreur dans lobfervation attronomique de M. Picard, avoit été reconnue \& confirmée en I739, fans que l'on eût fongé à former le moindre doute fur la jufteffe de fa mefure trigonométrique, \& moins encore fur ta mefure actuelle de Ia Bafe qui y fervoit de fondement; c'eft-ì-dire, fur Ia diftance du moulin de Villejuif au pavillon de Jivify, taquelle avoit été employée fur le pied de 5663 toifes. Ce ne fut qu'en $174^{\circ}$ quiil fut queftion de vérifier cette diftance. M. Caffini, aidé de M. l'Abbé de la Caille, la trouva alors plus courte que $M$. Picard ne l'avoit trouvée, de près de 6 toifes; c'eft-à-dire, d'environ une toífe par mille: différence qui doit influer néceffairement fur toutes les diftances conclues par cette Bafe. Cette erreur, fi elle eft réelle, ce que nous allons examiner, ne peut guère s'expliquer qu'en fuppofant que la Toife, qui avoit fervi d'étalon à M. Picard dans la mefure de fa Bafe, étoit trop courte d'environ $\frac{1}{1000}$ partie; ceeftà-dire, de près de $\frac{9}{10}$ de ligne : ce qui ne paroît pas aifé à concilier avec ce qu'on lit dans le livre de ta Mefure de la Terre de cet Auteur (art. IV, page ( 5); mais de peur qu'il n'arrive à notre Toife, conme à toutes les unefures anciennes dont il ue nous refle plus que le noull, nous l'attacherons à un original, lequel, étant tiré de la nature mếene, doit être invariable \& univerfel. M. Picard rapporte enfuite le détail de fon expérience du Pendule, \& conclut la 
longueur du Pendule à fecondes de 36 pouces $8 \frac{1}{2}$ lignes de $1 \mathrm{a}$ Toife de Paris, en prenant le milieu entre les oblervations faites en hiver \& en été, après avoir remarqué que la différence n'étoit que de la dixième partie d'une ligne.

Or cette longueur du Pendule de M. Picard diffère à peine de $\frac{1}{15}$ de ligne de celle qu’a trouvée $M$. de Mairan, qui l'a déterminée, avec le plus grand fcrupule, de 36 pouces $8 \frac{57}{100}$ ligne *; \& la conformité entre ces deux réfultats eft telle, que deux Obfervateurs, qui opéreroient en même temps dans le même lieu, \& en fuivant les mêmes procédés, n’oferoient fe flatter d'en rencontrer une plus grande; non feulement en faifant une expérience aufî délicate que celle dont il eft ici queftion, mais peut-ètre même dans la fimple comparaifon de deux mefures. Ceci pofé, on ne peut difconvenir, à moins de fe jeter dans les conjectures les plus hafardées, que la Toife, dont M. Picarda tiré fa mefure du Pendule de 36 pouces $8 \frac{1}{2}$ lignes, ne foit fenfiblement la même que la Toife de M. de Mairan; \& nous favons d'ailleurs que celle-ci a été faite par le même ouvrier, \& fur le même étalon que les deux Toifes qui ont fervi à la détermination du degré du Méridien (Part. 1, art. XXI, page 76 ); l'une fous l'E'quateur, \& l'autre fous le Cercle polaire.

D'un autre côté, fi l'on confidère que M. Caffini a mefuré jufqu'à cinq fois, \& en différens temps, la même Bafe, \& qu'il l'a trouvée conftamment de près de 6 toifes plus courte que M. Picard; fi on lit, fans prévention, le détail hiftorique de cette vérification dans le livre de la Méridienne de Paris vérifiée (article $I, \&$ Difcours prélim.), \& $\mathrm{f}_{\mathrm{I}}$ on pèle toutes les circonftances; on fera obligé d'avouer, quelque difpofition qu'on 
ait à tout révoquer en doute, quill n'eft guère poffible d'en former de raifonnables, fur l'exactitude de la nouvelle mefure.

M. Picard fe propofoit, comme il le dit dans l'endroit déjà cité de fa Mefure de la Terre, de laiffer en dépôt à l'Obfervatoire royal la longueur de la Toife, \& celle du Pendule à fecondes, telle quili les avoit établies. Si ce projet eût été exécuté, on auroit aujourd'hui, de la façon la plus évidente, le dénouement de la difficulté qui naît d'une part, de la conformité entre la mefure du Pendule par M. Picard, \& celle de M. de Mairan, conformité qui fuppofe l'égalité des Toifes quilis ont employées; \& d’autre part, de la différence d’une toife fur mille, entre la mefure achuelle d'une même diftance, par M. Picard \& par M. Calfini.

En attendant que le temps nous donne fur ce point quelque nouvelle lumière, $\mathrm{fi}$ toutefois il eft permis de l'efpérer, voici ce qui me paroît le plus vrai-femblable.

La capacité \& l'exactitude de M. Picard ne font contertées de perfonne: on ne peut, fans lui faire injure, fuppofer quil n’ait pas difcuté avec le foin quîl apportoit à toutes fes opérations, l'évaluation quil nous a laiffée de la longueur du Pendule en pieds, pouces \& lignes. Il n’a pas été dans le cas d'emprunter aucun fecours étranger pour vérifier cette lóngueur, il a dû opérer feul dains cette expérience, quiil a tant de fois réitérée: ainfr il n'ausoit pû, en cette occafion, fe tromper que par une négligence coupable, ou par une malhabileté dont if n'eft pas foupçonné. Il n'en eft pas tout-à - fait de même de ta longueur des perches de bois que M. Picard a employées pour la mefure de la Bafe: il eft bien vrai qu'on ne peut douter quiil n'ait apporté toute fon attention à les faire bien ajufter, \& ì 
Q $x$ à les vérifier fur la Toife de fer qui lui fervoit de mefure originale; mais jentrevois plufieurs caules d'erreur , fur léfquelles on étoit alors moins en garde quaujourd'hui, \& qui peuvent lui avoir fait trouver fa Bafe plus longue quelle ne l'eft en effet. Quoique le chaud \& le froid ne faffent pas changer, du moins fenfiblement, la longueur des mefures de bois, on n'ignore pas que la fécherefle \& l'humidité y produifent des variations confidérables, \& il feroit très-poffible que dans l'intervalle du temps où $M$. Picard ajufta \& vérifia fes perches fur l'étalon, \& celui où il les appliqua fur le terrein, elles fe fuffent defléchées \& raccourcies fenfiblement; mais voici quelque chole de moins conjectural.

M. Picard nous apprend qu'il fe fervit de quatre bois de pique, chacun de deux toifes, \& qu'ils fe joignoient à vis deux à deux, pour former des perches de quatre toifes (Mef. de la Terre, art. III, page I 3). Je fuppofe, \& on n'en peut douter, que chaque bois de pique, pris féparément, avoit la longueur précile que $M$. Picard avoit voulu leur domner; mais comme ces bois fe joignoient à vis deux à deux, pour former des mefures de quatre toifes, il eft aflez probable qu'en ferrant la vis, les deux bois de pique, de 12 pieds chacun, \& qui formoient une mefure de 24 pieds, fe comprimoient mutuellement ; \& que la mefure totale en étoit accourcie. Peut-être trouverat-on à cela plus que de la probabilité, fo l'on fait attention que la vis, qui joignoit les deux bois de pique, fuppofe une monture cylindrique de cuivre en forme de douille, dans laquelle entroit l'extrémité de ce bois, \& ma conjecture à cet égard, s'ent trouvée conforme à la vérité. J'ai appris que les perches de M. Picard fe font confervées long-temps à 
l'Oblervatoire avec leurs montures, \& qu'elles étoient en effet telles que je les fuppofe : or il eft clair, que pour donner à Ia perche, garnie de fa monture, la mefure exacte d'une toife, il falloit que le bois fût coupé plus court de quelques lignes que fa jufte mefure; \& ce bois, en fe defféchant, étoit difpofé à entrer plus avant dans la douille au moindre effort; ce qui devoit néceffairement raccourcir la mefure : \& l'on ne peut nier que celui quil falloit faire pour ferrer la vis qui joignoit les deux perches, ne fût très-propre à produire le même effet, auff bien que le moindre choc, à l'une des extrẹ́mités.

Enfin, M. Picard nous apprend, que les deux mefures ainfi ajuftées, \& de 4 toifes chacune, fe pofoient fur le terrein bout à bout alternativement, circonftance \& manière d'opérer qui domnent lieu de juger, qu'en approchant une perche de l'autre pour les faire fe toucher exactement, la dernière pof́e pouvoit faire reculer imperceptiblement la première; fur-tout ces perches étant rondes, légères, \& très-propres à gliffer fur un pavé uni, tel que celui d'un grand chemin des environs de Paris.

Je remarqu'erai ici en paffant, que c'eft pour prévenir unz femblable inconvénient, que nous avons toûjours employé dans nos mefures actuelles, trois perches au moins; en telle forte, que lorfqu'on en relevoit une, il en reftoit au moins deux fur le terrein : afin qu'en pofant la dernière, le petit choc, qui feul pouvoit nous affurer de fon contact avec la précédente, ne pût faire reculer celle-ci ; d'ailleurs cet accident étoit d'autant moins à craindre, que nos perches, longues de is ou de 20 pieds, \& dreffées d'équerre fur leurs quatre faces, avoient un pouce $\&$ demi fur deux pouces de gros; \& quainf 
elles étoient fujettes à un grand frottement, lors même qu'elles n'étoient foûtenues que fur deux appuis, \& qu'elles ne portoient pas de toute leur longueur fur le terrein où nous les appliquions immédiatement, quand cela étoit poffible.

La manière d'opérer de $M$. Caffini a dû pareillement le mettre à l'abri des caufes d'erreur que je viens d'indiquer dans Ia mefure de M. Picard, lefquelles étant conftantes, ont dû influer également fur fa première \& fur fa feconde mefure. M. Caffini a empíoyé quatre Règles de fer (Mérid. de Paris vérif. page 33 \& fuiv.), dont trois reftoient toûjours polées fur le terrein : le frottement caufé par leur poids, \& leur réfinance, ne donnoit pas lieu de craindre qu'en les approchant avec précaution (fur quoi M. Caffini ne s'en rapportoit qu'à lui-même), Ia dernière pofée fît reculer les trois précédentes. Ces Riègles étoient plates, \& ne fe joignoient point à vis comme celles de M. Picard: l'humidité ni la féchereflè ne pouvoient altérer leur longueur; \& quant aux variations caufées par le plus ou le moins de chaleur, M. Caffini opéroit le Thermomètre à la main, \& les différences qu'il a trouvées, \& qui n'ont jamais monté à 2 pieds fur la longueur totale de la Bafe, ont répondu aux alongemens que les différens degrés de chaleur, indiqués par le Thermomètre, pouvoient avoir caufés aux Règles de fer.

Enfin M. Caffini a non feulement réitéré fa mefure comme M. Picard, mais il l'a répétée jufquà cinq fois, en différens mois de l'année: ce n'eft donc que forcé par l'évidence, que M. Caffini a enfin abandonné une mefure qu'il avoit adoptće dans tous fes calculs, \& fur laquelle il n'avoit jamais foupçonné d'erreur. Aucune préoccupation n’a pû lui faire illufion 
252 MESUREDES TROIS PREMIERS

en cette rencontre; \& fi l'on pouvoit croire qu'il y en eút eu de fa part, il eft clair qu'elle n'eût été qu'en faveur de a mefure de M. Picard. Le fimple récit des faits fuffira pous met!re cette vérité dans tout fon jour.

Au mois de Juin r 739, M. Cafflui de Thury \& M. l'Abbé de la Caille, ayahnt vérifié les angles de l'ancienne Méridienne au Sud de Paris, jufqu'aux environs de Bourges, \& formé plufieurs Triangles nouveaux, dont un côté, de 7200 toifes, pouvoit être mefuré actuellement; ils le trouvèrent, par une mefure actuelle répétée deux fois fans un pouce de différence, de 7 toifes plus court qu'ils ne l'avoient conclu par le calcul des Triangles, en partant de la longueur fuppofée à la Bafe de M. Picard (Mérid. de Paris vérif. page 65 ). Ce manque d'accord, loin d'infpirer aucune défiance aux deux Obfervateurs fur la juftefle de la mefure de cette'Bafe, ne leur en fit naitre que fur l'exactitude de leurs propres opérations. Au mois de Novembre fuivant, Ia Bafe de Bourges fut mefurée une troifième fois par $M$. l'Abbé de la Ca:lle, avec de nouvelles perches, \& une nouvelle manière de procéder toute différente des précédentes; \& il trouva précifénıent la même łongueur que les deux premières fois (Mérid.vérif. ivid.). Au mois de Mars r 740, il entreprit de recommencer la mefure de fes angles, fur laquelle tomboient tous les foupçons; il forma une nouvelle Suite de Triangles, obferva tous les angles, \& retrouva la: même conclufion.

Alors on commença à foupçomer la Bafe de M. Picard; \& au mois de Juin I 740, M. Cafjini de Thury étant parti pous: continuer la Carte de la Méridienne en Flandre, M. Caffini te père, \& $M$. de la Caille, mefurèrent deux fois une diftance 
de $\$ 729$ toifes prefque dans l'alignement de M. Picard; \& par trois Triangles formés fur cette mefure, ils conclurrent ta difance du clocher de Brie-Conte-Robert à la Tour de 'Montlhery, plus petite d'une toife par mille qu'on ne la concluoit par la Bafe de M. Picard, à Jaqueile la nouvelle fut auff rapportée immédiatement; cependant ce ne fut qu'après en avoir répété la mefure une troifrème fois, que $M$. Caffini fit ce rapport à l'Académíe. Mais nétant pas encore pleinement convaincu lui-même d'un fait fi extraordinaire , il recommença une quatrième fois fon opération, au mois d'Août de la même année; \& retrouvant toûjours le même nombre, if demanda à l'Académie des Commiffaires, pour être témoins d'une cinquième mefure. Trois des Académiciens qui avoient fait le voyage du Nord, furent nommés. pour y aflifter, \& en rendre compte à la Compagnie. Cette. nouvelle mefure fe trouva conforme aux quatre précédentes; c'eft-à-dire, de $s 6 \varsigma 7$ toifes', au lieu de $566_{3}$, en la rapportant aux termes de M. Picara' (Mérid. de Paris, vérif. p. 37). Enfin. trois autres Bafes mefurées depuis, en Picardie, en Flandre, \& en Auvergne, \& lićes aux Triangles de la Méridienne, ne s'accordent quaux calculs faits fur le premier nombre: je Jaifle: maintenant au Lecteur à juger de quel côté eft l'erreur.

Si on balançoit encore à fe déterminer, voici un dernier fait, qui me paroît fuffre pour décider la queftion. Le regiftre. original de M. Picard, ayant été retrouvé en 1743 , trois ans. après la vérification de la Bale de Villejuif; on peut y voir encore aujourd'hui cottées de fa main, les longueurs de plufteurs. portions de fa Bafe, comprifes entre divers points quil défigne:telles qu'il les avoit trouvées en allant, \& enfuite en revenanto. 


\section{S4 MESURE DES TROIS PREMIERS}

De ces points, il y en a quatre qui font encore reconnoiffa. bles, \& dont les diftances ont toutes été trouvćes plus petites que par M. Picard, \& toûjours dans la même proportion (Mésid. de Paris vérif. page 38). En vain entreprendroit-on de jeter quelques doutes fur ce que les termes extrêmes de l'ancienne Bafe; favoir, le centre du moulin de bois de Villejuif, \& le coin du pavillon de Juvify, n'ont pas été reconnus avec affez d'évidence, malgré la recherche fcrupuleufe qui en a été faite; on n'en pourroit encore rien conclurre en faveur de M. Picard: car fuppofant qu'en effet il ne reftât plus le moindre veftige des deux termes de la Bafe, il n'en fera pas moins vrai, que la diftance de la Tour de Monthery au clocher de Brie, conclue par de nouveaux Triangles formés fur la nouvelle Bafe, prife prefque fur l'ancien alignement, s'eft trouvée de 13 1 o8 82 , au lieu de 13 1 $21^{\mathrm{t}}, 60$ quavoit trouvé $M$. Picard par fon calcul (ibid.), avec une différence de $13^{\mathrm{t}}, 28$ en moins; c'eft-à-dire, proportionnelle à l'erreur reconnue fur Ia Bafe; ce qui fournit, contre l'ancienne mefure, un nouvel argument fans replique.

Quoique le livie de la Méridienne de Paris vérifiée foit entre les mains de tout le monde, \& qu’on y voie le détail de la vérification de la Bafe de M. Picard; je n'avois pas laiflé d'être frappé de la force de l'objection prife de la conformité de la longueur du Pendule à fecondes, trouvée par M. Picard \& par M. de Mairan. J'ai donc voulu favoir à quoi m’en tenir fur un point, dont les conféquences font fi importantes pour la queftion de la figure de la Terre; \& jai cru que ceux qui ne cherchent que la vérité, me fauroient gré d'être entré dans cette difcuffion, en rapportant quelques faits qui 
n’avoient pas encore été publiés, \& qui ont contribué à éclaircir mes doutes : c'eft à ceux à qui il en refteroit encore à les diffuper entièrement; foit par la mefure actuelle de l'intervalle des deux termes de la dernière Bafe de M. Caffrii, tandis qu'ils fubfiftent avec évidence; foit en déterminant, par une nouvelle mefure, la diftance entre la Tour de Monthlery \& le clocher de Brie, ou un des autres côtés du Triangle que font ces deux mêmes points avec iObfervatoire, ou avec le clocher de Montmartre.

On a conftruit en $r 74^{2}$ un Obélifque de pierre, pour fervir de Terme feptentrional à la dernière Bafe de M. Caffuni : il en refte un à conftruire à l'autre extrémité. On y marquera, fans doute, la diftance des deux Termes, \& il eft de l'intérêt de l'Académie de ne permettre pas qu'il refte fur cela le moindre prétexte de douter.

M. Picard ne peut s'être trompé fur la mefure de fa Bafe, fans que toutes les diftances qui s'en déduifent ne fe foient reflenties de cette erreur; \& puifqu'elle monte à environ une toife d'excès par mille, if s'enfuit néceflairement, que de ce feul chef, il a dû faire la diftance des deux Cathédrales de Paris \& d'Amiens, trop grande d'environ 60 toifes, en la fuppofant de 59530 toifes, comme on la conclud de fes obfervations (Mef. du degré du Mérid. entre Paris \& Amiens, chap. $I$, page $V$ ).

Outre cela, on doit faire attention que M. Picard, comme il le dit lui-même, fe trouva preffé par le temps, \& forma fes derniers Triangles un peu à la hâte. Nous euffious bien voulu, dit-il (Mef. de la Terre, art. VI, page 48), avoir affez de temps pour chercher dans les plaines du Santerre quelque poinz 
propre pour fullir cette mefure par deux grands Triangles; mais la faifori étoit déjà trop avancée, de forte que nous fulumes obligés de nous contenter de ce qui fe trouvoit allx environs de Sourdon, oil il falloit féjountier pour prendre la hauteur du Pole. En effet, M. Picard, dans fes fix derniers Triangles, n'a mefuré que deux angles; \& ce qui tire fort à conféquence, il y en a parmi ceux qu'il n'a pas mefurés, queiques-uns de fort aigus, \& oppofés à de petits. côtés qui fervent de bafe, pour en conclurre de grands. Tel eft P'angle à Aluieus, entre Sourdon \& l'arbire de Morenil, qu'il a dû conclurre de $25^{\mathrm{d}} 26^{\prime} 5^{\circ} \mathrm{o}^{\prime \prime}$ (Ibid. page 49). Cet angle eft oppofé à un côté de 4822 toifes, \& il a fervi à en conclurre un de I I I 6 I toifes.

Dans le Triangle formé par les clochers de Coivrel, Mondidier \& Sourdon, l'angle à Coivrel n'a pas été obfervé; , mais feulement conclu par les deux autres, dont l'un, de i i 5 degrés, n’a pû être obfervé avec un Quart-de-cercle que par deux opérations qui exigeoient des réductions, \& elles n'ont pas été faites. Auffi l'angle à Coivrel, conclu par M. Picard de $37^{\mathrm{d}} 8^{\prime} \mathrm{o}^{\prime \prime}$ a-t-il été trouvé de $37^{\mathrm{d}} 6^{\prime} \mathrm{g} \mathrm{O}^{\prime \prime}$ par obfervation immédiate en I740 (Mérid. vérif. page I SI). M. Picald a donc dû conclurre la diftance de Sotrdon à Mondidier, \& par conféquent la différence des deux Parallèles, plus grande que la vraie.

Enfin en dernier lieu, $M^{\text {rs }}$ Caffini de Thury \& de a Caille, par une nouvelle Suite de Triangles mieux difpofés que ceux de $M$. 'Picard, de laquelle tous les angles ont été mefurés actuellement, \& qui, dans la partie depuis Clemmont en Beauvoifis jufqu'à Amiells, contient cinq Triangles prefque équilatéraux ( Mérid. de Paris vérif. pl. $X)$, ont conclu la diftance des Parallèles de Coivrel \& d'Amiens (pages 2760277 ), moindre de 45 toiles qu'elle 
réfulte des mefures de $M$. Picard. Dans cette erreur de 45 toifes, eft comprife celle d'une toife par mille, dont on a déjà tenu compte, \& qui eft d'environ i 9 toifes fur la diftance des Parallèles de Coivrel \& d'Amiens. Il refte donc feulement 26 toifes, quỉil faut ajoûter aux 60 déjà comptées, \& on aura 86 toifes de différence entre les deux mefures. II y a, fans doute, encore quelques autres erreurs du même fens, que ces Meffieurs n'auroient pas manqué de déterminer, fi les arbres de Boulogme \& de Moreuil euffent fubfifté dans le temps qu’ils ont vérifié la mefure de M. Picard.

Quoi qu'il en foit; par les nouvelles mefures de I 740, prifes avec d'autant plus de fcrupule, que $\mathrm{M}^{\mathrm{rs}}$ Caffini de Thury \& de la Caille s'attendoient à voir leurs opérations vérifiées de nouveau l'année fuivante, par les Acadéniciens qui avoient fait le voyage au Cercle polaire; fuivant le projet qui en avoit été formé dans l'Académie: par ces mefures, dis-je; confrrmées par deux Suites de Triangles qui donnent à peine une toife de différence fur 666 i o toifes (Mérid. de Paris vérif. pages $55 \&$ \& 5 ), la diftance de Paris à Amiens a été trouvée plus petite de 96 toifes (Ibid. $p . s^{I}$ ) que celle qui fe déduit des mefures de M. Picard; \& c'eft cette nouvelle erreur fur la mefure géodéfique, qui par un hafard fingulier, donne lieu à une nouvelle compenfation fi heureufe, que par le dernier zéfultat de M. Picard, fon degré du Méridien ne differe que de $14 \frac{1}{2}$ toif. de celui qui a été déterminé par les nouvelles mefures aftronomiques \& géométriques en 1739 \& i 740.

En effet M. Picard ayant, par un calcul défectueux, conclu l'amplitude de fon Arc (que nous avons réduit aux $\mathrm{Pa-}$ rallèles des deux Cathédrales de Paris \& d'Amiens), trop

$\mathrm{Mm}$ 


\section{MESURE DES TROIS PREMIERS}

grande de $7^{\prime \prime}$ (art. XXVIII page 245); \& ayant d'autre part trouvé la diftance des deux E'glifes trop longue de 9.6 toifes, équivalentes à 6 fecondes, il n’a dû trouver fon degré trop court que de la valeur d'une feconde : auffr l'a-t-il fixé à 57060 toiles, le même, à I $4 \frac{1}{2}$ toiles près, que celui qui a été déterminé de $57074 \frac{1}{2}$ toifes par les obfervations aftronomiques de $M^{\text {rs }}$ Maupertuis, Clairaut , Camus \& le Momier, \& les opérations trigonométriques de Mrs Caffini de Thury \& de la Caille (Mérid. de Paris vérif. page so. Voy. la Note).

\section{A R T I C L E X X X.}

Des divers rapports des axes du Sphéroïde terreftre, tirés de la comparaifon des divers degrés mefurés.

L'inégalité des degrés du Méridien, \& feur accroiffement de l'Équateur au Pole, étant conftatés par les mefures actuelles, \& les diverfes théories s'accordant jufqui ici à donner à la Terre une figure elliptique; voyons dans cette hypothère, ce qu'on peut tirer des différentes comparaifons des degrés du Méridien, pour en conclurre le rapport des axes de Ia Terre.

M. de Maupertuis, dans le tivre de la Figure de la Terre déternininé , \& dans les Mémoires de l'Académie de I 737, a donné une formule fimple \& commode, pour conclurre par Ia fimple mefure de deux petits arcs du Méridien, le rapport des deux axes de la Terre, fuppofée elliptique. L'élégance de la folution de M. de Maupertuis difpenfe de chercher une autre méthode pour réfoudre le même problème.

Soient $F \& E$ les longueurs données de deux petits arcs 
ćgaux du Méridien, par exemple, chacun d'un degré; foient $s \& \int$ les finus des latitudes moyennes de ces deux arcs; la formule générale, dans laquelle $D$ reprélente la différence des deux axes, eft $D=\frac{E-F}{3(E \mathscr{J}-F s s)}$. Et dans le cas où I'un des deux arcs mefurés eft très-voifin de l'Équateur, ia formule devient encore plus fimple, elle fe réduit alors à $D=\frac{E-F}{3 E \mathscr{J}}$. C'elt la feule dont nous avons befoin, pour comparer les degrés mefurés au Pérou, en France \& en Lapponie.

Si dans cette demière formule on fait $F=56750$ toifes, c'eft-à-dire, la valeur que j’ai attribuée au premier degré de latitude; \& fi on fuppofe que $E=57075$ toifes, prifes pour 1 longueur du degré du Méridien entre Paris $\&$ Amiens, en corrigeant l'arc \& la diftance (Voy. art. XXVII.p. 240), \& en fuppofant que le Parallèle de $49^{\mathrm{d}} 23^{\prime}$ partage ce degré en deux également; on trouvera la différence des deux axes de $\frac{1}{303,6}$; c'eft-à-dire, que le diamètre de l'E'quateur étant fuppofé de près de 304 parties, l'axe en aura un peu plus de 303.

La différence des deux degrés mefurés eft de 325 toifes dans la fuppofition précédente : fo on la réduit à 3 ro toifes, foit en donnant, avec M. Picard, i 5 toifes de moins au degré de France, tel que M. Picard \& M. Caffini l'avoient autrefois déterminé; foit en augmentant de is toifes le premier degré de latitude, ce qui eft, à très-peu près, conforme à la détermination de $M^{\text {rs }}$ les Officiers Efpagnols, nos Compagnons de voyage *; la différence des axes, au lieu d'être

* Ces Meffieurs fixent la longueur du premier degré de latitude à 56768 soifes (Obferv, aftr. y phys. Mautrid, 1749 , chap.V, page 295). 
26O MESURE DES TROIS PREMIERS

de $\frac{1}{303}$, fera de $\frac{1}{3} \frac{1}{38}$; c'eft-à-dire, que le numérateur de la fraction, qui exprime le rapport des axes, croîtra d'autant d'unités, quion aura retranché de toiles de la différence des deux degrés.

A quelque degré de France qu'on compare le premier degré de latitude, on trouvera toûjours, à peu près, le même rapport des axes. Si, par exemple, au lieu de choifir le $49^{\mathrm{d}} 23^{\prime}$, on aimoit mieux prendre le degré moyen entre les huit degrés un tiers, mefurés en France, lequel, fuivant les nouvelles mefures, eft de 57 o go toifes (Mérid. de Paris vérif. page I I 4), \& répond au Parallèle de $46^{\mathrm{d}} 43^{\prime}$, la différence de ce nombre à 5675 o toifes, valeur que j’affigne au premier degré de latitude, feroit précifément de 3 o o toifes, \& on trouveroit la différence des axes $\frac{1}{302,3}$, au lieu de $\frac{1}{303,6}^{\circ}$.

Maintenant fi c'eft au degré du Méridien, mefuré en Lapponie par les Ácadémiciens envoyés au Cercle polaire, que je compare mon premier degré de latitude de $5675^{\circ}$ toifes, fuppofant que le degré du Nord eft de $\$ 7438$ toifes, \& qu’il eft partagé en deux parties égales par le Parallèle de $66^{\mathrm{d}} 20^{\prime}$, fenfiblement le même que le Cercle polaire; je trouve que les axes diffèrent dans leur longueur de $\frac{1}{210}$ : \& fi ayant égard à la réfraction, qui a dû diminuer d'environ une feconde l'amplitude de l'arc mefuré en Lapponie, on fait ce degré plus court de 16 toifes, ou de $\$ 7422$ toifes, la différence des axes fera alors de $\frac{1}{2} \frac{1}{15}$ : de même que fi, fans rier changer au degré de Lapponie, on augmentoit le degré voifin de l'Écquateur de i 6 toifes, ou fi l'on fubftituoit au mien celui de $M^{\text {rs }}$ les Officiers Efpagnols.

Quant au rapport des axes de $177^{\text {à }}$ I 78 toifes, conclu 
par M. de Maupertuis (Mef. du deg. cntre Paris \& Amiens, p. v \& VI)., par la comparailon du degré du Méridien de Torneå à celui qu'il a auffr mefuré en France, entre Paris \& Amiens, avec $M^{\text {rs }}$ Clairaut , Camus \& le Monnier; c'étoit en fuppofant celui-ci de 57 I 83 toifes, tel qu'il réfultoit de la nouvelle amplitude comparcé à l'ancienne diftance, en tirant celle-ci des mefures trigonométriques de $M$. Picard. Mais fi on réforme cette diftance (Voy. art. précéd.), \& fi l'on compare le nouveau degré qui en réfulte de 57075 toifes, à celui qui coupe le Cercle polaire, après l'avoir réduit à caufe de la réfraction, à \$ 57422 toifes; on trouvera la différence des axes de $\frac{1}{132} ; \&$ on la trouveroit de $\frac{1}{145}$, fi on employoit le degré moyen de France de 570 go toifes, traverfé par le Parallèlle de $46^{\mathrm{d}} 43^{\prime}$.

Enfin on peut voir dans le livré de la Mérid. de Paris vérif. page I I 4, que le degré de longitude, mefuré en France fous le Parallèle de $43^{\mathrm{d}} 32^{\prime}$, \& comparé au degré de latitude correfpondant, pareillement mefuré, domne la différence des axes de $\frac{-1}{160^{\circ}}$

Je ne parle point des autres rapports, trouvés par les degrés du Méridien mefurés en France, \& comparés l'un à l'autre (Ibid.); ces degrćs étant trop voifins \& trop peu différens, pour qu'on puiffe tirer des conféquences affez füres de leur. comparaifon. Il fuffit-des réfultats précédens, pour prouver combien de variété il fe trouve entre les rapports des axes terreftres, conclus en comparant les longueurs des degrés mefurés à de grandes diftances. Nous venons de voir que ce rapport varie depuis $\frac{1}{13} \frac{1}{2}$ jufques à $\frac{1}{30}$. M. Newton, par fa théorie, fixoit l'inégalité des axes à $\frac{1}{23}-$.

Suivant un Theorème du même Auteur ( Phil. nats $M \mathrm{~m}$ iij 


\section{MESURE DES TROIS PREMIERS}

Princip, math. Lib. III, prop. XX), \& dont on doit la première Ménaires de démonftration ì $M$. de Maupertuis, les degrés du Míridien

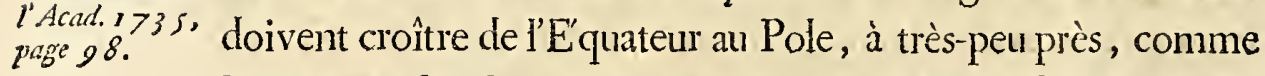
les quarrés des finus des latitudes; mais ni les mefures actuelles des degrés, ni les expériences de la longueur du Pendule fous différens Parallèles, ne peuvent fe plier à cette loi.

Ménoires de M. Bouguer a trouvé que les quatre mefures que nous I'Acad. 1744, page 297. avons des degrés terreftres, favoir; celles des degrés du Méridien, à Paris, à Torne̊a \& à Quito, \& celle du Parallèle du

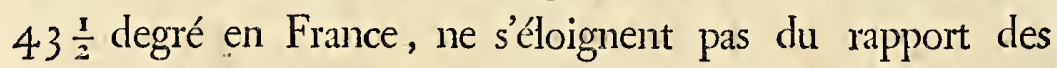
quatrièmes puiffances des finus de latitude. Mais n'y a-t-il pas lieu de craindre que la mefure d'un nouveau degré, que j’ore prévoir que nous aurons bien-tôt \& de bonne main, ne nous oblige à chercher un nouveau rapport, qui ne conviendroit peut-ĉtre pas mieux aux différences obfervées entre les longueurs du Pendule à différentes latitudes.

\section{A R T I C L E XXXI. \\ Conclufion.}

De u x grands hommes du fiècle paffé ont donné naiffance à P'opinion de Ia Terre aplatie vers les Poles. Huygens, par la feule théorie des forces centrifuges, de laquelle it eft l'inventeur, Newton, par des conféquences tirées de la même théorie, \& de celle de fon fyftème de la Gravitation univerfelle, devenu anjourd'hui la clef de toute la Phyfique célefte, ont établi l'un \& l'autre, que la Terre eft un Sphéroïde, dans lequel l'axe de rotation eft plus court que le 
diamètre de l'Équateur. Les expériences de la longueur du Pendule à fecondes à différentes latitudes, font autant de preuves de fait de cette conféquence, commune aux deux fyftèmes. Les trois mefures des degrés du Méridien fous les trois zones, confrimées par celle de deux degrés de longitude en France, ne permettent plus d'en douter; \& cette vérité fi long-temps conteftée, eft aujourd'hui univerfellement reconnue.

Mais autant la théorie, \& les mefures actuelles, s'àccordent à faire de la Terre un fphérö̈de aplati ver's les Poles, autant, comme on vient de le voir, different-elles fur la quantité de fon aplatiffement. Pour les concilier, on eft obligé d'avoir recours à diverfes fuppofitions; fur l'hétérogénéité des parties de Ia maffe terreftre, fur les diverfes denfités, épaiffeurs \& figures des couches dont elle peut être compofée, ou dont pourroit être pêtrí un noyau, qu'on fuppoferoit dans fon intérieur : en un mot, fur les différentes combinaifons des parties folides \& liquides, dont l'affemblage total forme la Terre. Tout ceci ouvre un vafte champ aux fpéculations les plus profondes, $\&$ offre le fujet d'un grand nombre de problèmes, fur tefquels nos plus grands Géomètres* fe font exercés. Trop à l'étroit dans l'enceinte du Monde phyfique, ils aiment à prendre l'effor dans la fphère des poffibilités : le réel \& l'intelligible font égalemént foûmis aux démonftrations mathématiques.

Avouons que jufqu'ici les hypothèfes, propofées fur la figure

* Figure des aftres, par M. de Maupertuis. Théorie de la figure de la Terre, par M. Clairaut. Préceffron des E'quinoxes, par M. Dalembert, Ch. $I X^{\prime}$ Voy. auffi dans les Recueils de l'Académie divers. Mémoires de M. de Matran, de M. Bouguer, \& des Auteurs précédemment nommés; 
de la Terre, font, ou purement géométriques, ou abfolument gratuites, ou font trop de violence aux obfervations, en cherchant à les accorder. D'ailleurs, toutes ces hypothèles ont pour bafe commune la parfaite reffemblance, \& l'uniformité de la courbure des Méridiens: elles fuppofent, je le répète, I ${ }^{\circ}$ Que tous les Méridiens fe reffemblent : $2^{\circ}$ Que leur courbure augmente ou diminue fuivant une loi uniforme \& régulière $(a)$; or il eft certain que ces fuppofitions ne font au plus que probables: car qui nous affure que les parties internes de la maffe terreftre font affez homogènes, pour quon puiffe tirer cette conféquence $(b)$ ? Les parties hétérogènes, au contraire, ne paroiffent-elles pas inégalement, \& irrégulièrement diftribuées à toutes les profondeurs connues? Je ne prétens pas pour cela que la Terre foit une mafle informe, bizarrement \& groffiè rement irrégulière; fuppofition auff contraire à toutes les obfervations quà toutes les théories; mais rien ne nous prouve jufqu'à préfent, que l'accourciffement fucceffif du rayon de Ia Terre, de l'E'quateur au Pole, au lieu de procéder uniformément, comme dans toute épèce d'elliple, ne foit pas fujet; par mille caufes phyfrques, à diverfes anomalies fous différens

(a) Ceci étoit écrit long-temps avant que j'euffe lû ce que $M$. de Buffon a dit fur cette matière dans fon ingénieux Syftème de la formation des Planètes, Hift. natur. Tom. I, page r65. J'y ai vû avec plaifrr que nous fómmes de même avis, quant à l'irrégularité poffible de la courbure du Méridien.

(b) La ligne' verticale pourroit changer d'un lieu à l'autre, par la même caufe à laquelle $M$. Newton attribue en partie les différences irrégulières dans Jes expériences du Pendule: \& hac difcrepantia partim à diffinilitudine partium internarum terrex . . . . oriri potuit. Phil. Nat. Princ. mathem. Tit. III, prop, $X X$. Voy. le favant Commentaire des RR. PP. le Sueur \& Jacquier. 
Méridiens ; \& fous le même Méridien, à des inégalités qui interromproient l'uniformité de fa courbure. Enfin quelque vrai-femblance qu'on veuille prêter à la fuppofition d'une courbure uniforme, \& femblable dans tous les Méridiens, cetie opinion a-t-elle plus de vrai-femblance que n'en avoit celle de la fphéricité de la Terre il y a un fiècle; \& depuis le moment où l'on a commencé à philofopher, jufqu'au temps dé M. Huygens, qui a le premier combattu ce préjugé philolophique avec des armes victorieufes?

La circularité apparente de lombre de la Terre dans les éclipfes de Lune; les mêmes hauteurs du Pole, obfervées après avoir parcouru des diftances égales, en partant d'une même latitude fous différens Méridiens; les règles de la navigation, qui dirigent d'autant plus furrement un vaiffeau, qu'elles font plus fûrement pratiquées, font les plus fortes preuves, \& peutêtre les feules qu'on puiffe alléguer contre le doute que j’ofe ici propofer; mais fans m'arrêter à affoiblir chacun de ces argumens en particulier \& d'autres femblables, je demande feulement s'ils ont plus de force pour prouver l'uniformité \& l'égalité de la courbure de la Terre, qu'ils n'en avoient pour prouver fon exacte fphéricité : or l'opinion fi ancienne \& fi univerfelle de cette exacte fphéricité eft reconnue aujourd'hui pour une erreur dont il feroit bien difficile de pouvoir douter: nous en fommes à peine fortis, craignons de tomber dans une autre, en donnant trop à la conjecture. Avant que de décider que la Terre eft un folide de circonvolution, attendons du moins que l'égalité de la longueur du Pendule à fecondes, fous la même latitude, foit confirmée par des expériences qui n’ont pas encore été faites; attendons que l'accroiffement régulier des 
266 MESURE DESTRAIS PREMIERS DEGRES, \&OC. degrés foit prouvé par des mefures qui nont pas été prifes fous le même Méridien à de grandes diftances ${ }^{*}$, pour prononcer que la courbe du Méridien n'a point d'irrégularité; contentons - nous aujourd'hui de croire que la Terre a une moindre courbure vers les Poles que vers l'Equateur, puifque le raifonnement \& toutes les mefures actuelles concourrent jufqu'ici à le prouver; mais laiffons au temps, \& aux obfervations multipliées, à décider de l'uniformité de cette courbure, ainf que de fa quantité.

* Les mefures des degrés en Lapponie, en France, \& en Amérique, fon fort éloignées d'être fous le même Méridien, \& il n’y a pas deux expétiences du Pendule faites fous le même Parallèle à différentes longitudes. 


\section{TABLE DES MATIERES}

\section{Contenues dans la Mefure des trois premiers degrés du Méridien.}

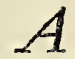

ABERRATION de la lumière, pages $127,139,170,220$. Voy. all $\sqrt{2}$ Colonne $\mathrm{V}$ des Tables d'Obfervations, \& pages $138,168,171,178,179$; $183,184,215,216$.

ANGLES. Avec quel foin a été prife Ja mefure des Angles, 14 \& 15. Angles de pofition obfervés entre les Signaux, Col. III de la Table des Triangles, 22 \& fuiv. \& 43 . Angles de hauteur \& de dépreffion apparente, même Table, Col. VI, \& pag. 46. Angles de pofition réduits à l'horizon, même Table, Col. VI1l, \& pag. 57. Pourquoi la Col. III de la Table eft intitulée Angles de pofition obfervés, 43. Angles verticaux: avec quel Quartde-cercle ils ont été obfervés, 46 . Angles verticaux ont été quelquefois déduits du calcul, 48. Théorie de ce calcul, 50. Pourquoi on peut fuppofer fans erreur tenfible que les trois Angles d'un Triangle réduits à I'horizon, forment un autre triangle rectiligne; 60 .

A N TOIN E de Ulloa (Don) l'un des deux Lieutenans de Vaiffeau, envoyés par le Roi d'Efpagne pour accompagner les Académiciens, 5. Se joint à Mirs Bouguer \& de la Condamine pour Ja mefure de la première Bafe, ibid. Mefure les angles des Triangles avec M. Bouguer, 12. Mefure la Bafe de Tarqui avec le même Académicien, 72. Publie à Malrid en 1748 une Relation du voyage, conjointement avec Don George Juan, 79.

A RC. Les plus grands font les plus avantagetux pour la mefure des degrés, x \& 2.Arcs (cordes des). Voy. corde.
Arc terreftre: \{a iongueur mefurée par les triangles de la Méridienne, г о r. Arc célefte (amplitude de l') compris entre les Parallèles des Obfervatoires de Tarqui \& de Cotchefqui, 221 . Autre détermination de cet Arc, 222. Même amplitude connue paŕ les obfervations fimultanées, 226 . Arcs différens du Méridien fous le Parallèle de Paris, mequrés en différens temps \& par différens Obfervateurs, 239. Comparaifon de l'Arc célefte mefuré par M. Ficard, a vec celui qui a été mefuré nouvellement en 1740 , 242.

Astronomie fournit feule les moyens de connoitre l'amplitude de l'arc du Méridien terreftre, ro6.

A XES. Différens rapports des axes de la Terre, tirés de la comparaifon des divers degrés mefurés, 258 .

\section{B}

$B_{\text {A s e d'Yarouqui, fa mefure, 4. Son }}$ profil, Pl. I, fig. $J$. La même inefuréc à différens niveaux, \& réduite à celus de Carabourou, 5. Ses deux différentes mefures ne diffèrent pas de trois pouces, $i b i d$. Réduite à la ligne droite inclinée, tirée d'un terme à l'autre, 9: Bafe de Tarqui, fa mefure, 71. Son profil, Planche I, fig. 2. Ses deux différentes mefures, prifes fur le terrein, s'accordent à moins de trois pouces près, 7 I. Son nivellement $\&$ fa réduction au niveau de Caraliourou, 73 \& fuiv. Comparaiton de la mefure de cette Bafe à fa longueur calculée par la Suite des Triangles, 85. Bafe de M. Picard. erreur dans fa mefure: Preuves, 247. Conjectures fur la caufe de cette erreur, 248 .

Nin ji 
Bernoulli (M. Daniel): fes expériences fur le rapport des flexions des barres de métal, 147.

Bouguer (M.) Ses obfervations dans l'Iite de l'Inca, 52. Dirige la conftruction de l'ancien Secteur, 108. Son procès verbal des Obfervations faites à Tarqui, 128 . Celui des $\mathrm{Ob}-$ fervations faites à Cotchefqui, 159. Table des Obfervations quil a faites feul à Tarqui, 178. Table de celles cuilil a faites feul à Cotchefqui, 183 . Fait conftruire un nouveau Secteur pour fes dernières Obfervations, 185. Son départ pour l'Europe, 2 19. Sa valeur du degré, 230. Voyez Bafe, Obfervations, Table, \&c.

Bradey (M.) découvre l'aberration de la lumière, \& fa Théorie, 127. Autle thćorie d'un nouveau mouvement apparent dans les étoiles, ilid.

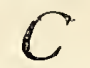

CA I l L E ( M. l'Abbé de la) publie en françois la nourelle Théorie de M. Bradley fur le mouvement apparent des Étoiles, caufé par la nutation de l'Axe terreftre, 127. Mefure avec M. Cafmi de 'Thury la diftance de l'aris à Amiens, 240, 241,256 \& fuiv. Détermine la valeur du degré du Méridien en France, ilid. \& 241. Mefure la Bafe de M. Picard avec M. Calfani, 24 (1, 252 . Vérifie la Méridienne de Paris à Barges, 2 5.2. Mefure une nouvelle Bafe trois fois, ibid.

Camus (M.) l'un des Académiciens qui ont mefuré le degré en Lapponie \& en France, $239,24 \%, 244$ \& 260.

CANONNIÈres \& Tentes employćes pour Signaux, $4 \mathrm{I}$.

Carabourou, terme boréal de la Bafe d'Yarouqui, 6. En le plus bas de tous les Signaux, \& tous les Trianglcs ont ćté réduits à ce niveau, 7 , $59 \& 228$. Sa hautcur au deffus du niveau de la $M e r, 52$. 'Température de l'air à Carabourou, $81 \& 82$.

CA.SS I N I dé Thury (M.) propofe de graduer un inftrument par les parties. aliquotes du rayon, 121. Mefure 1a longueur de l'arc du Méridien entre Paris \& Amicns, 241. Vérifie la Méridienne de Paris, 240. Détermine la longueur du degré du Méridien de Puis à Amiens, 240, 241, 256\& fuiv. Vérifie la Méridienne de Paris à Bourges, \&c. 252.

Chaleur (expériences nouvelles fur la. dilatation d'une Toife de fer par la), 5. En quelle raifon elle augmente ou diminue dans ta Province de Quito, 8o. Son effet \& celui du froid fur le Secteur, $14 x$.

Clajraut (M.) a étendu la théorie de l'aberration de la lumière, 127. Eft l'un des Académiciens qui ont mefuré l'arc célefte entre Paris \& Amiens, 231), 241, 244. Et l'un de ceux qui ont fixé la mefure du degré du Méridien en Lapponie, 260.

COLON ne première-de- la Table des Triangles: ce qu'elle contient, 40. Explication de la . $^{c}$ ibid. \& 41 . De la $3 .{ }^{c} 43$. De la $4 \cdot^{c} 44$. De la $5 .^{c}$ 45. De la 6.c 46. De la 7. ${ }^{\mathrm{c}} 49$. De la $8 .{ }^{c} 57$. De la $9 .^{c} 59$. De la $10 .{ }^{e}$ 62. De la 11. e $^{2}$ \& $12 .{ }^{c} 65$.

COMPARAISON des diverfes mefures du: degré près de l'Équateur, par les trois Académiciens, avec divers inftrumens, 23.0 \& fuiv. Les diverfes mefures du. degré en France, 2.39 \& fuiv.

Convergence des Méridiens exige une réduction dans la direction conclue des côtés du Triangle à la Méridienne, 64, 69.

Corde On fuppofe que les cordes des arcs qui forment les côtés des Triangles de la Méridienne, font ćgales aux arcs qu'elles foûtendent, \& pourquoì on le peut fuppofer, $6 \mathrm{I}$. Corde égale à une partie aliquote du rayon a tenu licu de graduation dans le Secteur, 17 \& fuiv.

Corrections diverfes qu'il a fallu faire aux Angles obfervés dans la mefure des Triangles, p. $16 \&$ luiv: Corrections cxtraordinaires, 20.

Cotchescui. Voy. Oljervatoire \& Obf, 
Cotes (M.) Sa théorie de afimatione errorum, employce par l'auteur, 91.

Co U P E du terrein de la Méridienne, Planche II, fg. 2, 54.

Coukbure (efiet de la) du rayon du Secteur, p. 147. Voy. Errear. Voy. Expériences.

Cuenca. Les Académiciens \& toute la Compagnie françoife y courent rifque de la vie dans une émeute populaire, 109.

\section{$D$}

Degré (mefure du). Sa détermination eft d'autant plus exacte qu'on mefure une plus grande étendue de terrein, 1. La mefure géodéficue du degré du Méridien établie dans ce Livre eft tirée des obfervations propres à l'auteur, $13 \& 233$. Précautions qu'il a prifes pour l'exécuter feul, 14. Détermination de la longucur du degré du Méridien aux environs de I'E'quateur, 227 \& 228. Valeur du degré du Méridien, tirée de quelcques obfervations particulières de l'aureur, 233 Valeur tirée de celles des deux Officiers ef pagnols, 234 . Inégalité des degrés du Méridien, 235. Sont plus petits près de l'Équateur que vers le Pole, pourquoi il fuit de là que Ja Terre eft aplatie vers les Poles? $23^{6}$. Les différentes mefures du degré du Méridien en France, 239. Longueur da degré du Méridien entre Paris \& Amiens, détermince felon M. Picard, ilident. Différence qu'y ont trouvé d'autres Affronomes, ibid. \& 240 . Longueur du degré du Méridien fous de Cercle polaire, 260 .

Dinection des côtés des Triangles. Voy. Méridienne. La direction de la Bafe étant connue par rapport à la Méridienne, connoître ta direction. des autres côtés des Triangles, 63.

Distance de deux lieux qui font féparés par un terrein incliné, mefurée horizontalement : par quelle voic fe peut réduire à la ligne droite, 5 . Diftance entre les Parallèles des Signaux, 23,
25 \& fuiv. Difance entre leurs Méridiens, itid. \& 65. De la caufe qui a pû augmenter la diftance apparente de l'ćtoile au zénith à 1 argui en 1732 , 152 \& fuiv.

Division de l'Ouvrage, 1. Divifion des degrés du Quart-de-cercle. Importance \& difficulté de fa vérification. Comment elle a étć faite, p. 17.

$$
\text { Ii }
$$

Ellipse. Si l'on peut condurre que ha Terre ait cette figure, par les feules obfervations faites jufqu'à préfent, $237 \& 238$.

ÉCUATION pour la Somme des trois Angles obfervés, 44. Ef? quelquefois nulle, ibid. E'quation is la lonoueur de la Méridienne pour une toife de différence fur la longueur de la $\mathrm{Bafe}$, $95 \&$ fuiv. Équations employées pour réduire les différentes obfervations au temps des obfervations fimultanées, 127. E'quation pour l'aberration de la lumière. Voycz Aberration. E'quation pour la nutation c'e l'axe terreftre, 127. Voy. auffis la fixième Col. des Tables d'Obfervations aftronomiques, citées au mot Table \& à celni d'Alerration. E'quation pour la préceffron des E'quinoxes, ibirl. $4 .{ }^{\mathrm{e} C}$ Col. des mêmes Tables.

Erreurs (denx fortes d') auxquelles eit fujéte la mefure des degrés, 1 \& 2 . Erreurs de chiffres. Moyen pratiqué pour les reconnoitre \& les vérifier, 15. Si toute erreur d'obfervation qui fera trouver trop long le dernier côté conclu des Triangles de la Méridienne, doit auffs néceffairement faire trouver trop loncue la Méridienne calculée, 87 \& Guiv. Si l'alongement qu'une erreur dans l'obfervation d'un angle produit dans un côté de Triangle conclu par le calcul, emporte néceffairement l'alongement de la portion correfpondante de la Mérid. 91 . Erreur d'une toife fur la longueur de la Bafe de Tarqui, conclue par le calcul des Triangl. quetle différence elle produit fur la longueur de la Méridienne dé Quito, 93 \& fuiv. Examen des difféNn iij 
rentes caufes d'erretur dans les obfervations, 141 \& fuiv. Caufes d'erreur dans les obfervations faites à Tarqui en $17 ; 9,142$ \& fuiv. Erreur cauf́e par la flexion du rayon du Secteur, en quel cas elle ne peut être d'une dangereufe conféquence, 143 \& fuiv. En quel cas elle peut devenir trèsconfidérable, 155 . De l'erreur porfible dans la détermination de la valeur du degré du Méridien, 229. Quelle erreur peut comporter la mefure aftronomique de M.rs Bouguer \& de la Condamine, ibid: Quelle erreur peut comporter la meruic géodéfique de l'auteur, 230 . Erreur dans la mefure aftronomique de M. Picard, 239 . Erreur dans la Bafe de M. Picard \& preuves, 246 \& fuiv. Erreur dans la mefure géodéfique de M. Ficard, indépendamment de celle de fa Bafe, 255. Par quelle compenfation les crreurs commifes par M. Picrzrd l'éloignent fi peu des nouvelles obfervations, 257 . Erreur caufée par l'aberration de la lumière. Voy. Aberration.

ESPAGNOLE (langue \& orthographe), ne peut rendre certains noms péruviens, 42 .

EsPAGNOLS (noms), écrits fuivant l'orthographe ef pagnole, \& pourquoi, - ibid.

Espagnols (Officiers). Voyez Don George Juan \& Don Antoine de Ulloa. Leur valeur du degré , 234 .

E'TOILE \& d'Orion, a principalement fervi à déterminer l'amplitude de l'Arc célefte, d'où eft tirée la mefure des degrés du Méridien, I $16 \& 12$ I.

E'TOlles d'Aninoïs \& du Verfeau, aufr obfervées pour la même fin, 122 \& fuiv.

EULER (M.) trouve un moyen pour perfectionner les Lunettes, 20 3. Détermine la longueur de l'efpace occupé par le foyer d'un verre de lunette, 205.

EXAMEN des caufes, \&c. Voy Erreurs.

EXPÉRIENCES fur la dilatation des métaux, $76 \&$ fuiv. Expériences nou- velles fur une Toife de fer, 77. Sur ta courbure que prennent des barres de fer par leur propre poids, 147.

$$
F
$$

Fig u R E de la Terre. Voy. Terre.

FIL-à-plomb. Le fil-à-plomb du Secteur étoit un fil de pite chargé d'un poids 1 92. Le poids n'a pas été plongé dans l'eau dans les dernières obfervation faites à Tarqui, \& pourquoi, 194. Accident fingulier arrivé au fil-àplomb, ibid.

Fiexion de l'Inftrument. Voy. Erreur.

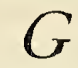

George Juan (Don) Lieutenant de Vairteau en Elpagne, envoyè par Sa Majefté Catholique, 5. A méfuré la Bafe d'Yarouqui avec M. Crodin, ilid. A mefuré les angles des Triangles de Ia Méridienne avec le même Âcadémicien, 12. A publié un Recucil d'obfervations, 79. Ses expériences avec M. Godin fur la dilatation des métaux, ilid Son calcul de la diftance des Parallèles des Signaux, 231 . A toûjours opéré fur le terrein conjointement avec M. Godin, ibid. Sa détermination de la longueur du degré du Méridien, $234 \& 259$.

GoDin (M.) déclare qu'il eft réfolu de faire à part l'obfervation aftronomique, yo6. Sa mefure géodéfrque, 12 \& 231 . Propofe de graduer un Inftrument par des parties aliquotes du rayon, 120 . Voy. Tentes.

$$
H \text {. }
$$

H A U T EU S \& abaiffemens refpectifs des Sign. Col Vll, 22 \& fuiv. Comment ils font défrnés dans la Table des Tr. 49. Hauteur abfóne des Signaux de la Mérid., , \& fuiv. Hauteur de Curathourou, le plus bas des Signaux, au deffus du niveau de la Mer, 53. Hauteur du Sol de quelques licux de la Province de Quito, \& des montan gnes les plus remarquables, 56 . 


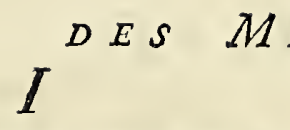

$I_{M A G E S}$ différentes d'un objet au foyer de l'objectif d'une Lunette. Divers Obfervateurs voyent une image différente, \& le même Obfervateur ne voit pas toûjours la même, 196 , 197 \& fuiv.

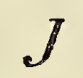

J UAN (Don George). Voy, George.

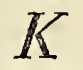

Kermadec (M. de) obferve avec les Académiciens qui mefurent l'amplitude de l'arc du Méridien entre Paris \& Amiens, 241 .

\section{$L$}

Letrtres qui diftinguent les différens Quarts-de-cercle dont on s'eft fervi pour les mefures, $16 \& 43$.

LIMBE. Flexion de l'Inftrument dans le plan du limbe, 143. Limbe applani, 191.

Limites de l'erreur à laquelle eft fujet le calcul pour la réduction de la Bafe à la liogne droite, 9. Limites des erreurs poffibles dans la détermination de l'amplitude de l'arc, 239. Dans la détermination de la longueur de l'arc, 232. Dans la détermination de la valeur du degré, ibid.

LONGUEUR des côtés oppofés aux Angles obfervés, fujet de la cinquième Colonne, 22, 24\& fuivantes, \& 45. Longueur des Bafes mefurées fur le terrein. Voy. Bafe. Longueur des côtés horizontaux réduits au niveau de $C_{a}$ rabourou, 23,25 \& fuiv. D'où fe peut conclurre la longueur de la Méridienne, $6_{2}$. Longueur totale de $\mathrm{Ia}$ Méridienne, 104.

I UNETTE fixe du Quart-de-cercle: vérification de fa pofition, 16 . Lunette: fon parallélifme au plan dia Secteur, 148 \& fuiv. Le foyer de la Lunette varie fuivant les différentes vûes, 199. Et pour la même vûe fuivant les différens états de l'atmofphère, 201 .
Marra n (M. de). Sa mefure du Pendule à Paris, $257^{\circ}$

Maupertuis ( $M$. de). Ses remarques fur la flexion du rayon du Secteur, 1 46 \& 157. A rendu fenfibles les conféquences de la décroiffance des degrés du Méridien quant à l'aplatiffe-. ment de la terre vers les Poles, 236 . Sa mefure de l'amplitude de l'arc cntre Paris \& Amiens, \& du degré du Méridien en Lapponie. Voyez Mefure. Ses formules appliquées aux diverfes mefures des degrés du Méridien, 238 , 258 \& fuiv. Démontre le premier un Théorème de M. Newton, 262.

MÉRIDIEN. Inégalité de fes degrés. Voy. Degré.

Méridienne. Table du calcul des Triangles de la Méridienıe de Quiro, 21 \& fuiv. Dircétion des côtés des Triangles par rapport à la Méridienne, Colonne $\mathrm{X}$ de la Table des. Triangles, $23 \&$ fuiv. \& 62. Longueur totale de la Méridienne, réduite au niveals, élevé de 1226 toifes au deffus de la mer, 104 .

MESURE géométrique de la Méri. dienne, particulière à l'autcur, 13 . Moyenne entre celles de M.rs Godin \& Bouguer, 232. Mefure de l'amplitude de l'arc du Méridien, compris entre les Parallèles de Paris \& d'Amicns, par $\mathrm{M}^{\text {rs }}$ de Maupertuis, Clairaut, Camus \& le Monnier, comparée à celle de M. Ficard, 242 \& fuiv. Mefure de la longueur du même arc, par $M \mathrm{rs}$ de Thury \& de la Caille, 256 . Examen de la mefure de la Bafe de $M$. Picard, $246 \&$ fuiv. Nouvelle mefure de cette Bafe, par M. Cafini, répétée cing fois, $242,2\{2$ \& fuiv. Mefure du degré du Méridien en Lapponie, 260.

Micromètre : l'auteur s'en eft toû́jours fervi dans la mefure de fes Angles, \& de deux manières différentes, 14. Détermination de la valeur des parties du Micromètre da Secteur de 12 pieds de rayon, 113 . Remarque 
nouvelle fur l'ufage du Micrometre, 209.

Monnier (M. Je) confirme par fes obfervations celles de M. Bradley fur l'aber ration de la lumière, 127 . Mefure l'arc célefte entre Paris \& Amiens, $239,241,244$. Prouve par plufieurs inverfions du Secteur qui avoit fervi aux obfervations en Lapponie, qu'il ne varioit pas par le trantport comme les inftrumens ordinaires, 245 . Mefure le degré du Méridien en Lapponie \& en France, $239,241,244$, 260.

Morainville (M. de) feconde M. de Ia Condumine dans fes dernières obfervations à Tarqui en $1742,189$.

\section{N}

Newton (M.) démontre ta diverfe réfrangibilité des rayons de lumière, 202. Détermine la longneur de l'efpace occupé par le foyer des verres de lunettes, 205 . Déduit l'aplatiffement de la Terre vers les Poles, de fa théorie de fa gravitation jointe à celle des forces centrifuges, $26_{3}$.

Nivea de Carabourou, Sa hauteur au deffus de la mer, 52. Terme auftral de la première Bafe près de Quito, inférieur au niveau du termse feptentrional de la feconde Bafe, \& de combien, 80. Réductions au niveau. Voyez Réduction.

No m $S$ des lieux où étoient pofés les Signaux , 2i, 24 \& fuiv. Explication de la Colonne où font contenus ces noms, 40 \& fuiv. Pourquoi ces noms font écrits felon f'orthographe françoife, quoiqu'ils foicnt Indiens polır ta plufpart, 41 \& fuiv. Noms efpagnols confervés dans leur orthographe, 40. Noms différens donnés au même lieu par les Indiens, d'où viennent ces différences, $i b: d_{\text {s }}$

Nuta'rion de l'axe terreftre (E'quation pour Ia ), 127. Voyez Aberration \& Colonne 6.e des Tables aftronomiques, citées illid.

$\mathrm{O}_{\text {Bjets }}$ propres à tenir lieu de Sígnaux: communs en France, \& mainquoient dans le pays oir les trois Âcadémiciens ont opéré, 40 .

Observateurs (noms tes) \& de leurs affifans, 5, 12 \& 14.

Observations (lifte des diverfes) faites pour déterminer l'amplitude de J'arc célefte, 121 \& fuiv. Premières Obfervations à Quito en 1737, 121 . Premières à Tarqui, au Sud de la Méridienne, en 1739 , 122 . Premières à Cotchefqui, au Nord de la Méridienne, en $1740,123$. Secondes à Quito en 1740 \& 41, ibid. Secondes à Tarqui, au Sud de ta Méridienne, en 174 1., 124 . Troifièmes \& dernières obfervations à Quito en $174^{2}, 125$. Troifièmes \& dernières à Tarqui, au Sud de la Miéridienne, en $1742 \& 1743$, correfpondantes à celles de Cotchefgui, \& fimultanées, ibid. Secondes \& dernières à Cotchefqui, au Nord de 1 a Méridienne, en 1742 \& 5743 , correfpondantes à celles de Tarqui, \& fimultanées, ilid. De quelles obfervations on a tiré la valeur du degré du Méridien, 126. Détail des premières obfervations faites à Tarqui en 1739, dans le procès verbal qui en fut fait fur les lieux, 128 . Table des obfervations de $\varepsilon$ d'Orion, faites à Tarqui en 1739 , \& réduites au premier Janvier 1743,138 . Des caufes qui peuvent nuire à la jufteffe des obfervations, 141 . Détail des premières obfervations faites à Cotchefqui en $174^{\circ}$, contenues dans un proces verbal fait à Quito, 159. Table des obfervations de \& d'Orion, faites en commun à Cotchefqui en $174^{\circ}, 168$. Détail des obfervations de la même étoile à Quito, 17ı. Remarques fur ces obfervations, ibid. Table des ob* fervations de l'étoile \& d'Orion, faites à Tarqui en 174r par M. Bouguer, I 78: Remarques fur les obfervations de la Table précédente, 180. Détail des dernières obfervations faites à Cotchefgui 
$D E S M A T I \dot{E} R E S$.

vij

Cotchefqui, 183. Remarques fur ces obfervations, 184 . Détail des dernières obfervations faites à Tarqui par l'auteur, 215 . Remarques fur ces obfervations, 217 . Obfervations $\mathrm{fi}_{\mathrm{I}}$ multanées, faites aux deux extrémités de la Méridienne, 225 . Préférables" aux autres \& pourqquoi, 226 .

Observatorre de Tarqui, en quel endroit placé, 113. Obfervatoire de Cotchefqui, 159. Leur fituation par rapport aux Signaux de Chinan \& de Cotchefqui, 103.

ORDRE \& plan des Triangles de la Méridienne de Quito, 22, 24 \& fuiv. Explication de la Colonne qui les renferme, $4^{\circ}$.

Orion (étoile e d') fert aux Obfervateurs à mefurer l'amplitude de l'arc du Méridien, 116 . Pourquoi la diftance de cette étoile au zénith, a paru plus petite que la vraie en 173?, 154. Obfervations de l'étoile $\varepsilon$ d'Orion. Voy, Obfervations.

Oyambaro, terme auftral de la Bafe, 6. Sa hauteur au deffus de $C_{\text {arabou- }}$ rou, terme boréal de la même Bafe, 82 . La température de f'air d'Oyambaro, ibid.

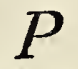

Parallaxe des fils au foyer de la Lunette, 195 . Variable pour les différentes vûes, \& pour la même vûe en différens temps, 196. Quand aperçue, 198. Sa théorie, $200 \&$ fuiv. Manière de l'éviter, 207.

Parallélisme: on a eu égard au défaut de parallélifme de la lunette au rayon du Quart-de-cercle dans fes obfervations des Angles, 16. Examen du parallélifme de la lunette au plan du Secteur de 12 pieds, 149 \& fuiv. $\&$ iश1. Ce parallélifme fe peut vérifier par l'inverfion de l'Inftrument, ainfi que la pofition de la lunette, 149 \& fuiv.

Pendule. Voy. Ficard, Mairan.

PERChEs qui ont fervi à mefurer les Bafes fous l'Équateur, comparées cha- que jour à la Toife de fer, 82 . Quelles \& comment employées! Conjedures fur celles de M. Picard. Remarques fur celles de M. Cafjemi, 248-25i.

Picand (M.) Erreur dans fa mefure aftronomique, 239 \& fuiv. Examen de fa Bafe \& de fa mefure géodéf que , 2468 fuiv. Sa mefure du Pendule à Paris, 247.

POUCES (quelques) de plus ou de moins fur la longueur de la Bafe ne font d'aucune importance pour la mefure du degré, 9. Les deux différentes mefures des Bafes d'Yarougui \& de Tarqui ne different que de deux ou trois pouces, $5 \& 72$.

PrÉCAUTIONS particulières prifes dans les dernières obfervations faites à Tarqui en $1742 \& 1743,187$.

Précession des Équinoxes (équation pour la ), 127 . Voy. auff E'quation.

PRocÈs verbal des obfervations faites à Tarqui en 1739, 128. Des obfervations faites à Cotchefqui en 1740, 159 \& fuiv.

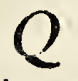

QuarT-de-cercle : avec quels Quartsde-cercle ont été mefurés les Angles. $13,15 \&$, 6. Erreurs de leur divifion. Voy. Divifion \& Vérifications. Comment ont été diftingués dans la Table des Triangles les angles obfẹrvés avec I 6, 43, 44\& 48 . Quel eft le Quartde-cercle défigné $d$, \& celui défigné $\boldsymbol{e}$. $43 \& 44$.

Quito (Province de), pays de montagnes, 5 . Hauteur da fol de Quito, 56. Détermination des points des Triangles de la Méridienne à l'égard de Quito, 66. Tour de l'églife de la Merci à Quito, point de fection de la Méridienne \& de la Perpendiculaire de Quito, \& pourquoi choiff。 67. Température de l'air à Quito, 80.

$$
R
$$

RÉDUCtion at centre, correftion néceffaire aux Obfervations des angles Oo les différens Quarts-de-cercle, 15 , 
des Triangles, 19. Réduction des angles à un plan horizontal, \& de quelle conféquence elle étoit, 46 . Ce qu'elle fuppofe, ibid. Réduction des angles oblervés en différens plans à l'horizon, fujet de la huitième Colonne, 23,25 \& fuiv. \& 57 . Comment fe fait cette réduction, 57 \& fuiv. Procédé que l'autenr a fuivi dans la réduction de tous les côtés des Triangles horizontaux au même niveau, 59. Réduire un angle à t'horizon, ce que c'eft, 60. La Réduction a été faite par la Trigonométrie iphéricque, \& pourquoi ? 6ı. Réduction de la diftance des Parallèles des deux Signaux extrêmes à celle des deux $\mathrm{Ob}$ Eervatoires de Cotchefqui \& de Tarqui, 103 Réductions des obfervations des étoiles à une même époque, 126 . Méthode pour conclurre l'amplitude de l'arc mefuré du Méridien fans aucune réduction, 223 . Réduction du degré au nivcau de la Mer, 228.

REFRAETion des objets terreftres, cuelle eft la quantité de la réfraction qui altère les deux angles de hauteur \& de dépreffion de deux objets vûs réciproquement, 5o. Réíraction aftronomique, de combien elle change les difances obfervées de l'étoile au zénith, $222 \& 225$.

RÉFRANGIBILITÉ diverfe des rayons de lumière, comment elle peut occafionner de la diverfité dans les obfervations, 202 \& fuiv.

RÉSULTAT des nouvelles expériences faites fur l'alongement d'une Toile de fer par la chalcur, 78 . Réfultat des fuppofitions faites fur les errcurs qui peuvent faire juger la Mérid. trop longue, 74 . Réfultat dess différentes fuites d'obfervations, voy. les Tables d'obfervations, $138,168,171,178,183$, $215,225, \& 219$ \& fuiv. Réfultat des obfervations correfpondantes aux deux extrémités de l'arc, 222. Réfultat des obfervations fimultanées, faites pour déterminer l'amplitude de l'arc du Méridien, 222,225\& fuiv. Réfultat des fuppolitions faites fur les erreụrs qui ont pû fe gliffer dans la détermination de l'amplitude \& de la longueur de l'arc, 232 .

\section{$S$}

$S_{A}$ \& LE noir métallique que l'aiman attire, $18 y$.

SECTEuR. Changemens faits au. Sectcur de douze pieds, apporté de France, , 106. Defcription de ce Secteur dans fa nouvelle conttruction, $109 \&$ i ro. Arc tracé fur ce Secteur, 116. Moyens qu'on emploie pour tracer l'arc du Secteur, 118. Effets du froid \& du chaud fur ce Sefteur, 141. Effet de la fiexion de ce Secteur dans le plan du limbe, 143. Dans un plan perpendiculaire à celui du limbe, 147 \& fuiv: Pourquoi le Secteur, tel qu'il a été tranfporté de France à Quito, n'étoit pas propre pour les obfervations qui devoient déterminer. Pamplitude de l'arc du Méridien , 172. Changemens faits au Secteur par M. Bouguer, 160 , 169,182 . Autre Secteur conftruit à Quito pour les dernières obfervations de M. Bouguer à Cotchelqui en 1742 , 185 \& fuiv. Secteur de M. Graham, $127,144,155,244$. Seclcur démonté \& raffermi par $M$. de la Condamine, 187 \& fuiv. Sufpenfion du Secteur perfectionnée, 188 \& fuiv.

SignaUX. Attention des Obfervatcurs dans l'ordonnance \& la difpofition des Signaux qui devoient terminer leurs Triangtes, 10. Noms des lieux où ids étoient pofés, Tab. Col. II, 22,24 \& uiv. Leurs hauteurs \& leurs dépreffions refpectives, même Tab. Col. VII, 23,25 \& fuiv. Diftance entre leurs Parallèles, ilind. Col X 1. Diftance entre leurs Méridiens, ibid. Col. XII. Défignés par lettre initiale dans le calcul de chaque Tr. de la Tab 40. Signaux artificiels, pour'quoi les Obfervateurs ont été obligés d'y avoir recours.' De quoi conftruits. Les tentes \& canonnières employées à cet ufage, 41. Signaux : la plufpart de coux cmployés par les trois Obfervateurs ont 


\section{$D E S \quad M A T I \grave{E} R E . S . \quad$ ix.}

des noms indiens, ibid. Pourquor le nombre qui exprime leur diftance fe trouve répété dans la Table, 45. Dcux Signaux peuvent paroittre réciproquement abaiffés fous l'horizon, 50 . Table de la hauteur abfolue des Signaux, 55 . Quel a èté le plus bas de tous, 228 .

SPHérö̈de. La terre eft un Sphérö̈de aplati vers les Poles, $62,2,36$ \& fuiv. Divers rapports des axes du Sphéruïde terreftre, tirés de la comparaifon des divers degrés mefurés, 258 .

SutTe (de Triangles): on en a formé deux pour mefurer la Méridienne, 12. De combien de Triangles chacune, \& en quoi elles different, ibid. \& fuiv. Autre Suite non employée, 86. Suite d'obfervations, ce qu'on a appelé dif. férentes Suites d'oblervations, 122.

Surrositions (fauffes), employées à propos facilitent les calculs tans induire en erreur, 62. Ce qui réfulte des fuppofitions forcées d'erreurs poffrbles dans la déterminatior du degré, 232 .

$$
7
$$

Table des Triangles, à quel deffeir l'auteur l'a dreffée, 3. Table des erreurs du Quart de-cercle, 18. Table du calcul des Tr. de la Mérid. de Quiro, 22 \& fuiv. Table de la hauteur des Signaux de la Mérid. de Quito au deffus du niveau de la Mer, 55. Explic. des col. de la Table des Triangles, 40 \& fuiv. Table de la hauteur des Signaux de la Méridienne de Quito. 'Table des diftances des Signaux à la Méridienne \& à la Perpendiculaire fur la Méridienne de la Tour de la Merci de Quito, réduites au niveau de $\mathrm{Ca}$ rabourou, le plus bas de tous les $\mathrm{Si}$ gnaux, 68 \& 69. Table d'obfervasions de l'étoile \& d'Orion à Tarqui en 1739,138 . Remarques fur les obfervations de cette Table, 139. Table d'obfervations de l'étoile \& d'Orion, faites en commun à Cotrhefqui en $1.740,168$. Remarques far les obfervations de cette Table, 169. Table des obfervations de la même ćtoile à
Quito en dcux différens endroits, \&c. 171. Remarques fur la $T$ able de ces obfervations, ibid. Table des obfervations de la mêmc étoile, faites à Tarqui en 174.1 par M. Bouguer, 178 \& 179. Remarques fur ces obfervations, 180 \& fuiv. Table des diffances de Ia même ćtoile au zénith de Cotchefqui, obfervées par M. Bouguer à la fin de $1742,183 \&$ \& 184 . Remarques fur les obfervations de cette Table, 18 . Table des diftances de l'étoile $\varepsilon$ d'Orion au zénith de Tarqui, obfervées par l'auteur en 1742 \& 1743 . réduites au premier Janvier 1743 , 2 I5 \& 216 . Remarques fur les obfervations de cette Table, 217 \& fuiv.

TARQUI (plaine de), terrein uni propre aux obfervations, 17. Targue (Bafe de). Voy. Bafe. Température de Yair dans fa prairie, 83. Diftance de fon Obfervatoire au Sign. 104. Tarqui (obfervations faites à ). Voy. Obfervations. Obfervatoire de Tarqui, 113.

Température. Voy. Chaleur. Cuito. Tarqui. Yarouqui.

TENTES \& Canonnières employées pour Signaux, $4 \mathrm{r}$.

TERrE. Queftion de fa non-fphéricité décidée, 234 \& fuiv. Sa figure efk celle d'un Sphéroïde aplati, 62, 237 . Preuse, 236 . La quantité de fon aplatiffement ne peut le déterminer que par des hyphothèfes, $2 \sigma_{2}$.

Thermomètre de $M$. de Recumur. 79, $142-8$ yafim.

Thury (M. de). Voy. Caffini.

Tolse. Defcription de la Toife de fer qui a fervi à régler les mefures, 75. Modèle dépofé à l'Académie, 76 . Expériences pour conftater fon alongement par le chaud, \& la contrac tion par le froid, $76 \& 77$. Réfultat. des expérijences faites fur l'alongement d'une Toife de fer par la chaleur, $7^{8}$. Pourquoi le réfultat des expériences faites à Quito par M. Godin \& Don George Juan ne s'accordent pas à celles de $\mathrm{M}$. de la Condamine fur la dilatation d'une Toife de fer, 79 , 
note (II). Comparaifon de la longueur de la Toife lors de la mefure des deux Bafes, 80. Quel degré marquoit le Thermometre à Paris lorfque la Toife de fer qui a fervi aux mefures, a été étalonée, 85.

Transversales : l'auteur ne les a jamais employées dans la mefure de fes Angles, 14.

Triangles. Expofition du fyltème de Triangles formés pour mefurer la Méridienne de Quito, ro. Attention que I'on a eue dans leur difpofition, 1 I. Triangles, on en a formé deux Suites qui donnent trois Mefures trigonométriques complétes, \& indépendantes les unes des autres, I 2 \& fuiv. outre une autre Suite non employée, 86. Table des Triangles de la Méridienne de Quito, 21 \& fuiv. Les Triangles auxiliaires inférés dans la Suite des Triangles de la Méridienne, rapprochent la longueur de la Bafe de Tarqui calculée, de fa longueur mefurée, 86.

TRIGONOMÉTRIE fphérique: moyen le plus commode pour réduire les Angles à l'horizon, 57, 60,61.

\section{$U$}

$\mathrm{U}_{\text {L Lo A A }}$ (Don Antoine de). Voyez

\section{$M A T I E$ E $R S$.}

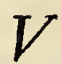

VERGUIN (M.) mefure la première Bafe avec Mrs Bouguer \& de la Condamine. Mefure la feconde Bafe avec M. de la Condamine. Fait ia Carte du terrein de la Méridienne. Oblerve avec les mêmes Académiciens à Tarqui en 1739, \& à Cotchelqui en $174^{\circ}, 137$. Ses certificats, 167 .

Vérifications des erreurs des divifrons du Quart-de-cercle employé aux obfervations géodéfiques de l'auteur. 17. Vérification double du Secteur. Voy. Parallétifme.

VOYAGES entrepris pour déterminer la figure de la Terre. Quel en a été le motif, 237 .

$$
Y
$$

Yaroueui (Bafe d'). Voy. Bafe. Température de l'air dans da plaine d'Yarouqui, 81 \& 82.

$$
7
$$

ZÉ ITH. Manière d'obferver la diftance d'une étoile au zénith fans le fecours des divifions ordinaires, i 6 . Comment fe conclud la diftance apparente de l'étoile au zénith par cette méthode, 1 2.8. Vérification de la Lunette au zénith, 149 \& fuiv. 


\section{Fig. I \\ Percu.}

Oraubaro Ton

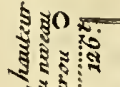

* 7 s......

क है.

Carabourou Terme Nord

Fig a croul

Ch

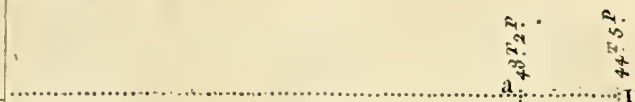

Tirm

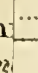

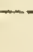

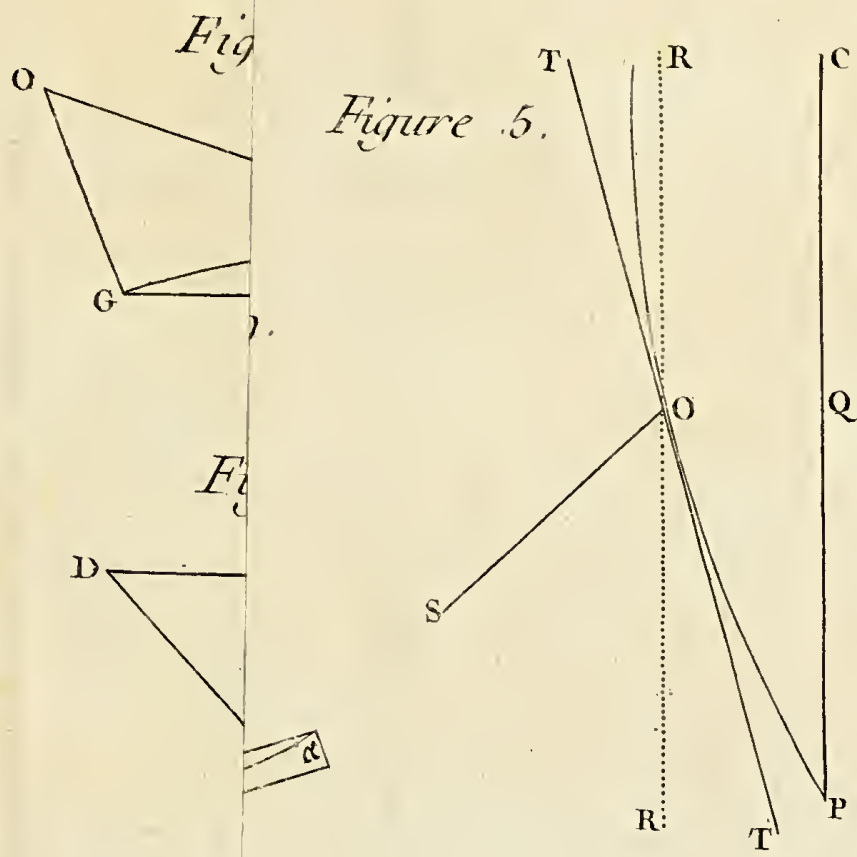

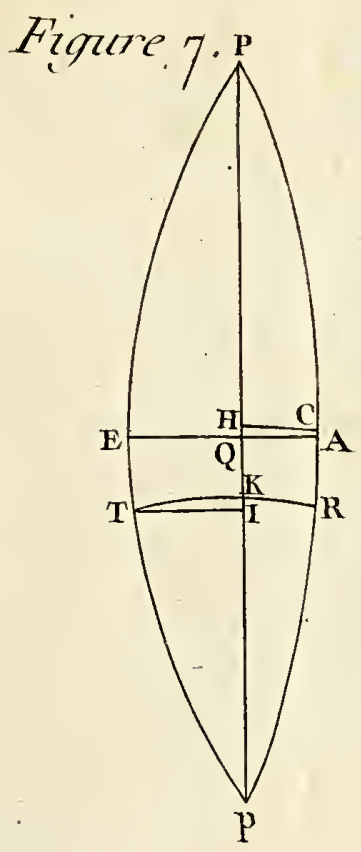





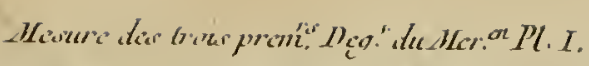
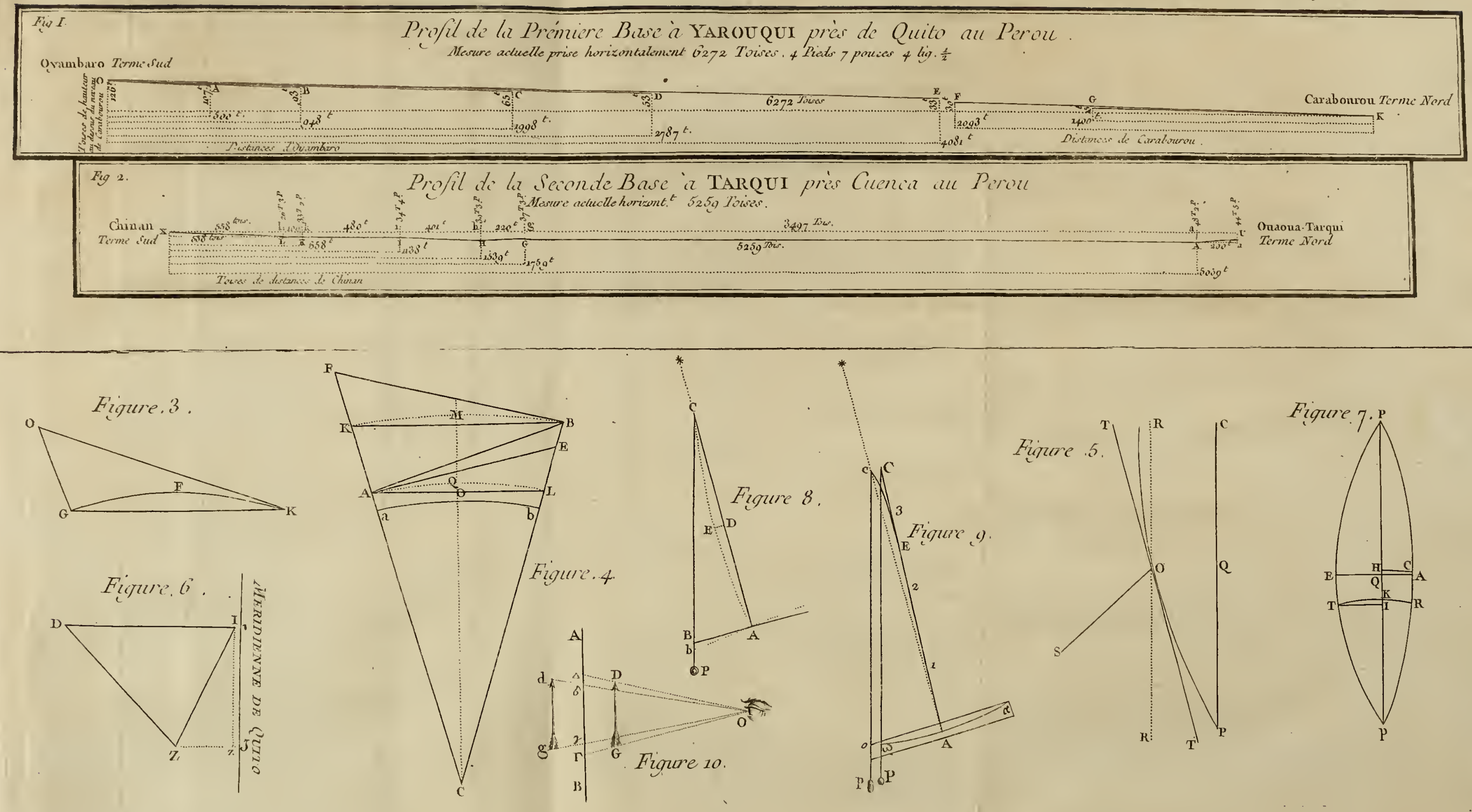


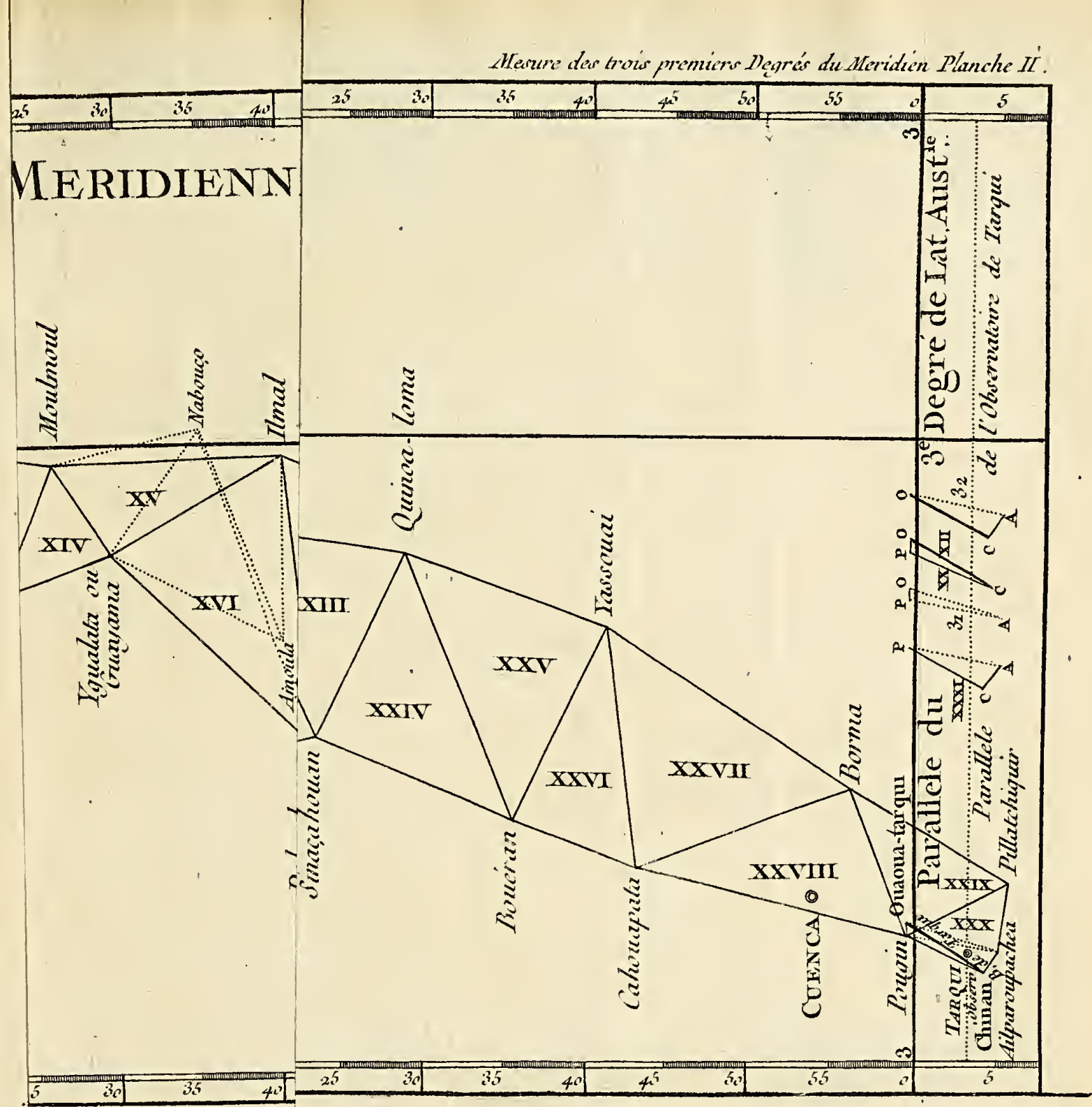

\section{ridienne de}

jangles; et des Mteliere des Andes

Eihalle des as mil Toiser.

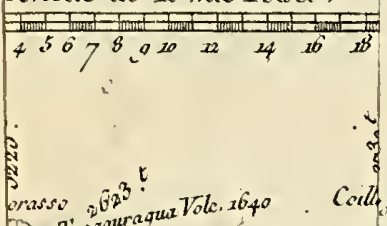

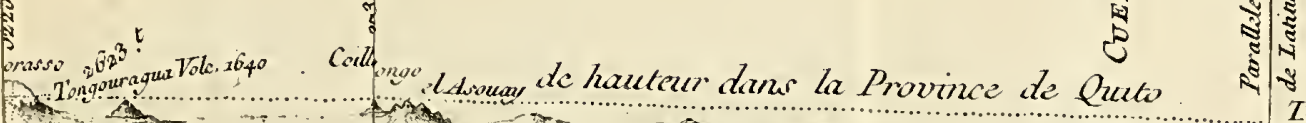

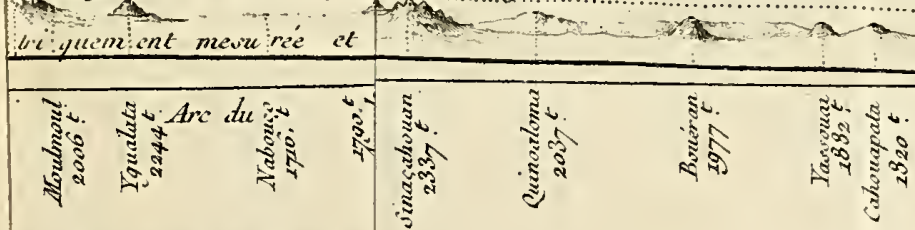






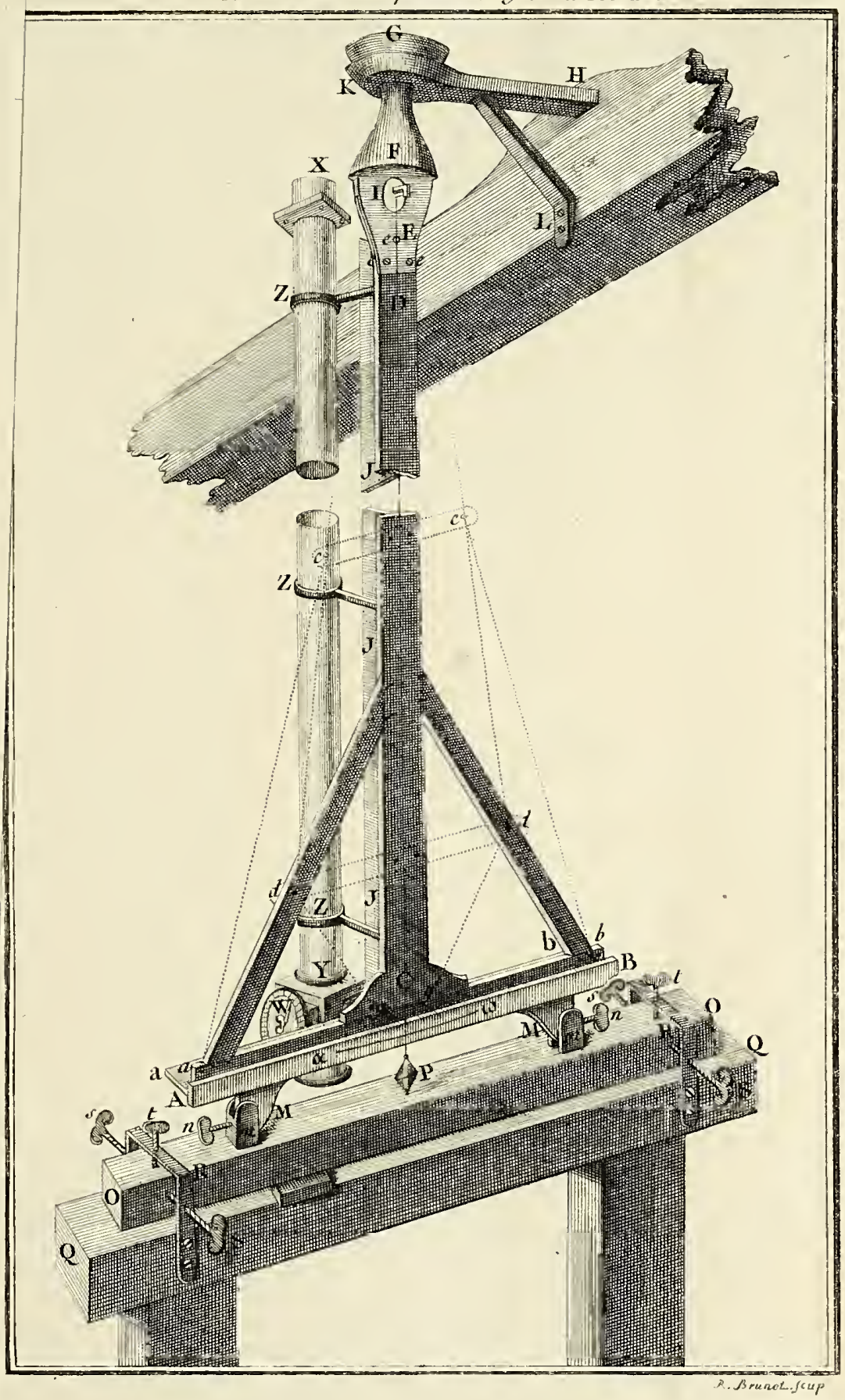



Masure dear irour premurvo Degrás du Merulasn Planshe III.

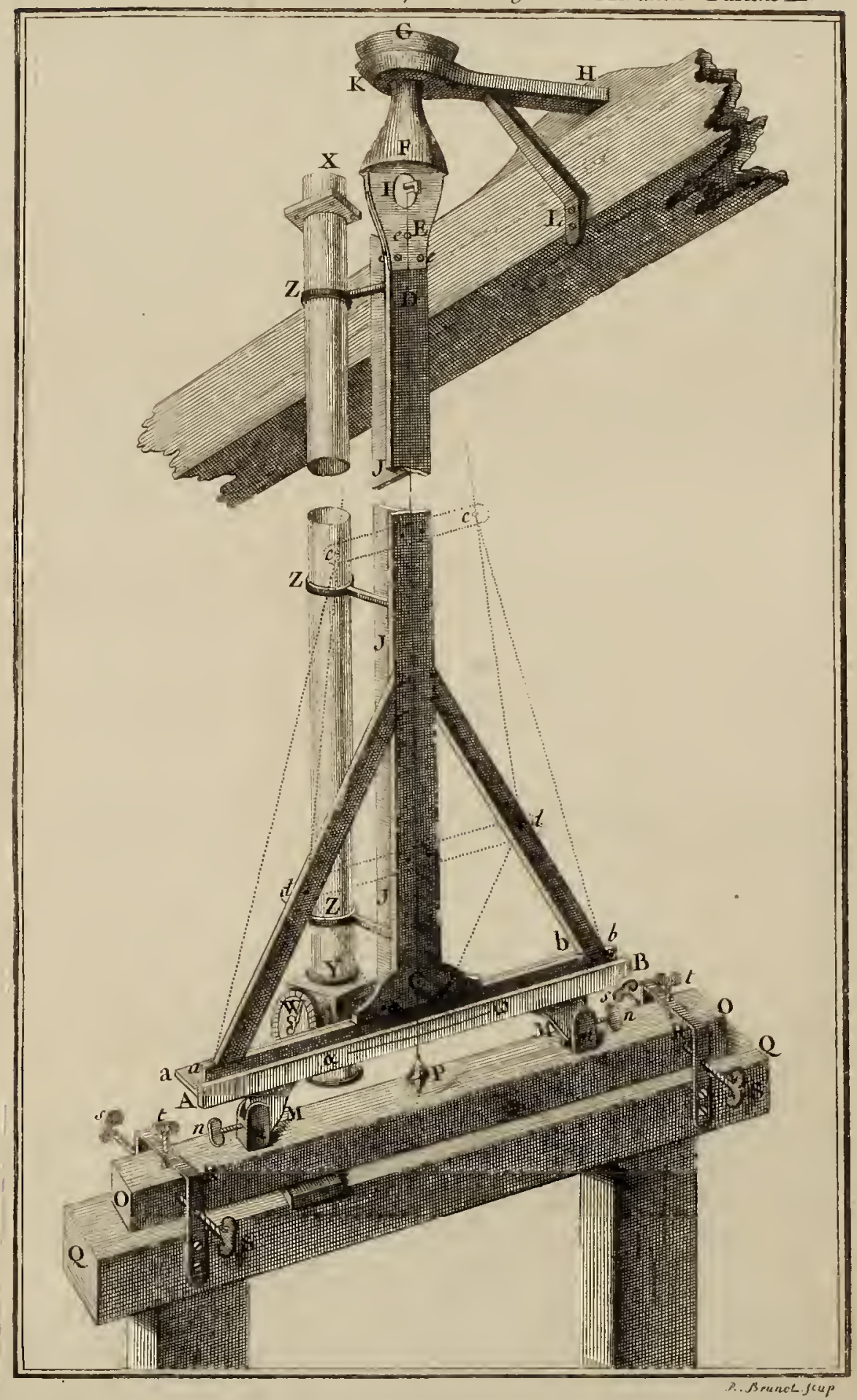



$i$

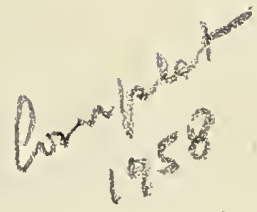




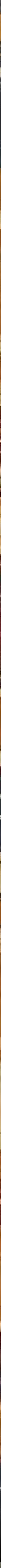




\section{$y=1.00 \cdot 0$}

$300 \%$ in

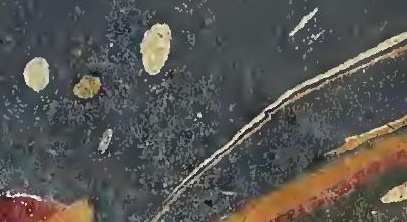

$\therefore \because 10$. 05

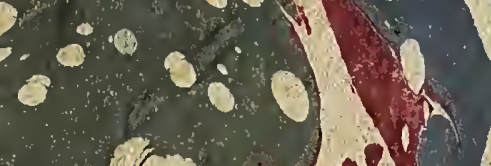

$\circ r$

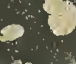

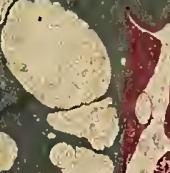

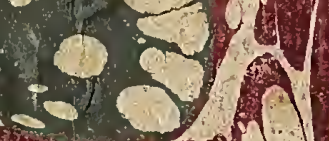
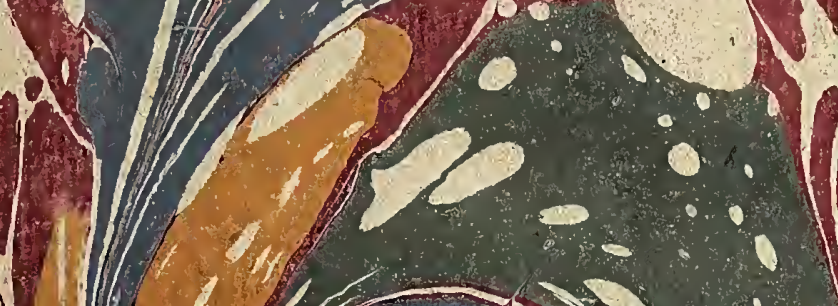

41

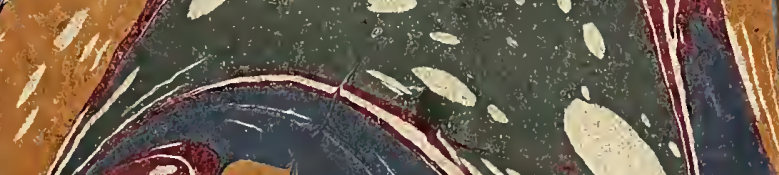

-)) ${ }^{2}$

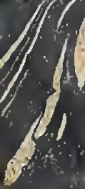

$1,6 \% 5$.

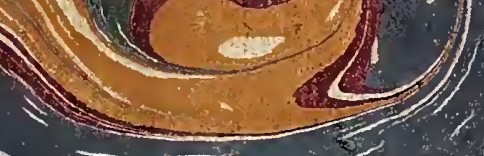

$1 / 1=$

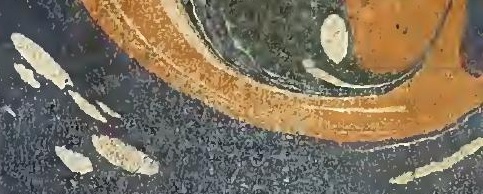

at

12: $:$

19) 8

$0.0 \%$ $2=-110$

- $a^{\prime \prime}=1$

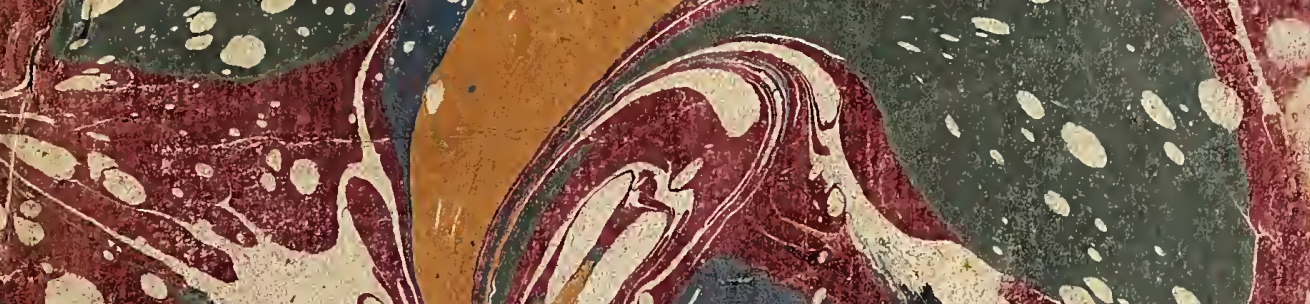

0

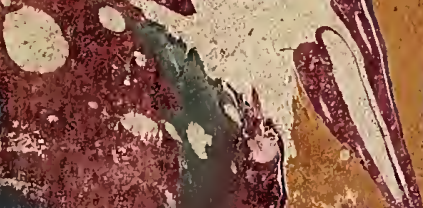

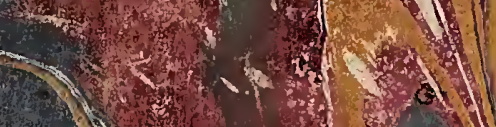

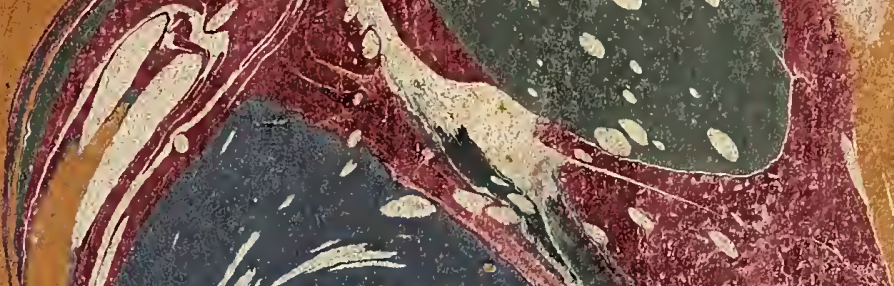
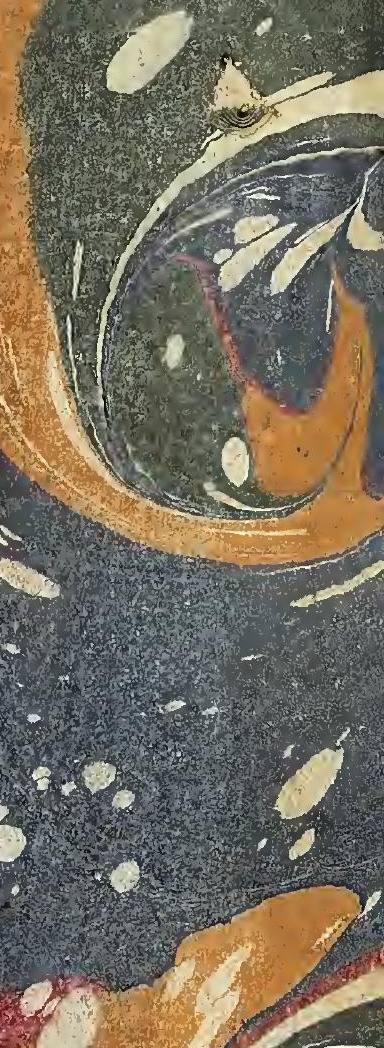
